\title{
Abstracts of Papers Presented at the Sixth Biennial Scientific Meeting of the Asia Pacific Paediatric Endocrine Society
}

\author{
Paul Hofman \\ Liggins Institute, New Zealand \\ Correspondence should be addressed to Paul Hofman, appes@willorganise.com.au \\ Received 10 November 2010; Accepted 10 November 2010 \\ Copyright ( $) 2010$ Paul Hofman. This is an open access article distributed under the Creative Commons Attribution License, \\ which permits unrestricted use, distribution, and reproduction in any medium, provided the original work is properly cited.
}

\section{Oral Presentations}

Kaichi Kida

Thursday 18th November 2010

11:30AM-12:30PM

\section{Bone Strength, Geometry, and Muscle Function in the Lower Leg of Healthy Adolescents}

Julie Briody, ${ }^{1}$ Gill A ten Hoor, ${ }^{1,2}$ Sarah P Garnett, ${ }^{1}$ Charmaine Tam, ${ }^{1}$ Chris T Cowell, ${ }^{1}$ and Craig Munns ${ }^{1}$

${ }^{1}$ The Children's Hospital at Westmead, Westmead, NSW, Australia

${ }^{2}$ Maastricht University, Maastricht, The Netherlands

This study aimed to (a) examine the relation between bone strength, geometry, and muscle function in the lower leg of healthy 17-year-old adolescents and (b) examine the influence of birth weight, body composition, physical activity, calcium intake, and vitamin D in mid-childhood on bone parameters at age 17 years.

The 162 adolescents ( 87 girls), mean age \pm SD $16.8 \pm 0.2$ years, recruited were participants of the nepean longitudinal study, a contemporary birth cohort. Anthropometry data were collected using standard techniques. Bone parameters and cross-sectional muscle area were measured by pQCT at the $66 \%$ distal tibia site (nondominant leg). The polar stress strain index (pSSI), a measure of bone strength, was calculated from $\mathrm{pQCT}$ data. Muscle force and power were measured using a Leonardo Mechanography ground reaction force plate. Body composition (DXA), physical activity (questionnaire), calcium (food record), and vitamin $\mathrm{D}$ were measured at 8 years (midchildhood).

Tibial pQCT data showed that boys had a greater periostcal circumference ( 90 versus $85 \mathrm{~mm}$ ), higher cortical area (369 versus $330 \mathrm{~mm}^{2}$ ), and bone mineral content (BMC) ( 970 versus $871 \mathrm{mg} / \mathrm{mm}$ ), but lower cortical density (BMD) (1075 versus $\left.1090 \mathrm{mg} / \mathrm{cm}^{3}\right)$, all $P<.001$. Bone strength (pSSI), bone strength adjusted for tibia length (pSSI/tlength), muscle force, and power were also higher $(P<.001)$ in boys compared to girls. Overall, assessing parameters measured at birth, 8 , and 17 years, regression analysis indicated that $42 \%$ of the variation in PSSI/tlength was explained by muscle force at 17 years and $90 \%$ of the variability of tibial BMC was explained by sex and muscle area at 17 years and leg BMC measured in mid-childhood. Using bivariate analysis, muscle force at 17 years was positively associated with birth length $(r=0.26, P=.001)$, DXA bone parameters $(r=0.32$ to $0.58, P \leq .001)$, and lean tissue mass $(r=0.65, P \leq .001)$ measured at 8 years. Similarly, muscle area was associated with DXA bone parameters $(r=0.26$ to $0.38, P \leq .01)$ and lean tissue mass $(r=0.43, P \leq .001)$ measured at 8 years.

These data support the importance of the musclebone unit during the growing years for bone development. To maximize bone strength during adolescence, increasing muscle force throughout the growing years should be encouraged. 


\section{Lentiviral Vector-Mediated Knockdown of SOCS3 Enhances Leptin-Induced Stat3 Activation in Rat Glioma Cells}

\author{
Liu Zheng Juan, Bai XueMei, Sui XiaoYan, \\ and Yan Dong
}

\section{Department of Pediatrics, Second Hospital Affiliated to Dalian Medical University, Dalian, China}

Objective. The overexpression of SOCS3 gene was one of the important mechanisms of leptin resistance through inhibition of pathway downstream of leptin-activated JAKSTAT signalling; therefore, suppression of SOCS3 is a potential therapy for leptin resistance in obesity. The present study was to investigate the role of SOCS3 knockdown in leptin-induced Stat3 activation in vitro. Methods. We first constructed the lentiviral RNA interference (RNAi) vectors of rat SOCS3 gene and evaluated the effects of silencing SOCS3 gene expression by siRNA in rat glioma cells. Three gene-specific sequences of siRNA targets for SOCS3 were designed, and the complementary DNA containing both sense and antisense oligonucleotides was synthesized. After phosphorylation and annealing, this doublestranded DNA was cloned, respectively, to corresponding pRNA-lentivector-VGFP to construct pRNA-Lenti-SOCS3VGFP recombinants with U6-containing promoter, target sequence, and Poly terminator. Three target sequences were correctly cloned to corresponding pRNA-lentivector-VGFP, which was confirmed by electrophoresis and DNA sequencing, and the effects of RNAi to reduce gene expression were further evaluated by real-time PCR in transfected rat glioma cells. To examine the role of SOCS3 knockdown in leptininduced Stat 3 activation, the glioma cells were cultured using DMEM containing 10\% FBS and transfected, respectively, with pRNA-Lenti-SOCS3-VGFP, or pRNA-Lenti-ControlVGFP by Lipofectamine 2000 after $80 \%$ cell confluence; 48 hours later, cells were stimulated or not with $100 \mathrm{nM}$ leptin for 10 minutes, cell lysates were prepared and proteins were quantified, and Western blot analyses were performed for leptin-induced Stat3 phosphorylation. Results. The target sequence of siRNA-SOCS3 was successfully cloned to pRNAlentivector-VGFP, and the RNAi protocol specifically reduced expression of SOCS3 mRNA by approximately $80 \%$ compared with controls in transfected rat glioma cells. Stats3 activation was significantly greater in cells transfected with pRNA-Lenti-SOCS3-VGFP compared with their controls. Conclusion. SOCS3 knockdown by siRNA enhances leptin receptor signaling, and this molecule is a potential target for therapeutic intervention of leptin resistance and dietinduced obesity in vivo.

\section{Altered Chemokine Levels in Children with Type 1 Diabetes and Its Relation to C-Peptide, HbAlc, and Disease Progression}

\section{Xin Ying, Chen Xiao Juan, Yang Min,} and Tong Ya Jie

Department of Pediatrics, Shengjing Hospital of China Medical University, Shenyang, China
Aims. Cell-mediated immunity and proinflammatory cytokines are implicated in the pathogenesis of type 1 diabetes. The aim of this study was to investigate whether circulating chemokines involved in T-helper 1 (monocyte chemoattractant protein-1 (MCP-1) and macrophage inflammatory protein-1a (MIP-1a)) and Thelper 2 (macrophage inflammatory protein- $1 \beta$, MIP-1b) autoimmunity were changed in children with type 1 diabetes at onset and followup and to explore the associations of MCP-1, MIP-1a, and MIP-1b with metabolic status and beta-cell function. Methods. Serum MCP-1, MIP-1a, and MIP-1b were measured in 50 children with newly diagnosed Type 1 diabetes, 30 age-matched healthy children with no family history of diabetes. In the diabetic children, additional serum samples were obtained at 3,6 , and 12 months after diagnosis. Serum levels of chemokine MCP-1, MIP-1a, and MIP-1b were measured by enzyme-linked immunosorbent assay (ELISA). At the same time fasting blood glucose, Cpeptide, and glycosylated hemoglobin (HbA1C) in diabetic children were routinely detected. Results. Serum MCP-1, MIP-1a levels were significantly higher in newly diagnosed diabetic children than in controls $(P<.05, P<.01)$, but serum MIP-1b levels in diabetic children had no significant difference compared with controls. In the type 1 diabetic patients at followup, serum MCP- 1 and MIP-1a were still significantly higher than controls at 3 and 6 months after diagnosis $(P<.01, P<.05)$ and decreased to nearly controls at 12 months $(P>.05)$. While serum MIP-1b levels did not change significantly. MCP-1 concentrations were negatively associated with C-peptide at 0,3 , and 6 months after diagnosis $(P<.01, P<.01$, and $P<.05)$. MIP-1a concentrations were negatively related to $\mathrm{C}$-peptide in newly diagnosed diabetic children $(P<.01)$. Conclusion. This study demonstrated children with type 1 diabetes onset existence of Th1/Th2 immune imbalance. Chemokine MCP-1, MIP-1a were involved in the process and progress as the condition persists, suggesting that chemokine MCP-1, MIP-1a might play an important role in the pathogenesis of type 1 diabetes in children. Blockage of chemokines by therapeutic agents may provide a new therapeutic target to ameliorate disease progression in type 1 diabetes.

\section{Retinal Venular Tortuosity and Urinary Albumin Excretion in Young Persons with Type 1 Diabetes}

\author{
Paul Benitez-Aguirre, ${ }^{1,2}$ \\ Muhammad Bayu Sasongko,, 3 Maria Craig, ${ }^{1,2}$ \\ Jie Jin Wang, ${ }^{3,5}$ Ning Cheung, ${ }^{3}$ Alicia Jenkins, ${ }^{6}$ \\ Tien $Y$ Wong, ${ }^{3,7}$ and Kim Donaghue ${ }^{1,2}$ \\ ${ }^{1}$ Institute of Endocrinology and Diabetes, \\ The Children's Hospital at Westmead, Australia \\ ${ }^{2}$ Discipline of Paediatrics and Child Health, The University \\ of Sydney, Australia \\ ${ }^{3}$ Centre for Eye Research Australia, Royal Victorian \\ Eye and Ear Hospital, University of Melbourne, Australia \\ ${ }^{4}$ Department of Ophthalmology, Gadjah Mada University, \\ Yogyakarta, Indonesia
}


${ }^{5}$ Centre for Vision Research, University of Sydney, Australia

${ }^{6}$ Department of Medicine, University of Melbourne, Australia

${ }^{7}$ Singapore Eye Research Institute, National University of Singapore, Singapore

Aims. To study the relationship between retinal venular tortuosity and renal dysfunction in young persons with type 1 diabetes (T1D). Methods. This was a prospective longitudinal study of 536 patients with T1D of at least 2-year-duration, aged 12 to 20 years. Retinal vascular tortuosity was measured at baseline from retinal photographs using computer-based analysis following a standardized protocol. Urinary albumin excretion rate (AER) was obtained at each visit from three consecutive timed overnight urine specimens. Longitudinal analysis was performed using generalized estimating equations with baseline arteriolar and venular tortuosity, gender, diabetes duration, $\mathrm{HbAlc}$, diastolic BP SDS and BMI SDS as explanatory variables. Results. At baseline 412/536 (77\%) participants had normal AER. Over a median (IQR) followup period of 4 (2-6) years, 154/412 (37\%) developed incident AER elevation. In multivariate analysis reduced retinal venular tortuosity was associated with elevated AER at any time point (OR1.6, 95\% CI 1.1-2.4, the lowest versus highest quartile). Conclusion. Lower retinal venular tortuosity was prospectively associated with early renal dysfunction. Reduced venular tortuosity may reflect underlying increases in hydrostatic pressure and oxidative stress associated with arteriolar and venular dilatation. Retinal venular tortuosity may be a useful noninvasive predictor of early microvascular complications in T1D.

\section{Clinical and Molecular Characterization of Noonan Syndrome and Related Disorders}

\author{
Han-Wook Yoo, 1,2,3 Beom Hee Lee, 1,2,3 \\ Jae-Min Kim, ${ }^{2}$ Gu-Hwan Kim, ${ }^{2,3}$ Hye Young Jin, ${ }^{3}$ \\ and Jin-Ho Choi ${ }^{3}$ \\ ${ }^{1}$ Department of Pediatrics \\ ${ }^{2}$ Genome Research Center for Birth Defects and \\ Genetic Disorders \\ ${ }^{3}$ Medical Genetics Clinic and Laboratory, \\ Asan Medical Center, University of \\ Ulsan College of Medicine, Seoul, Korea
}

Noonan syndrome (NS) and related disorders (NSLDs) are caused by dysregulation or gain-of-function mutation in a member of RAS-MAPK signaling pathway. With more causative genes identified, their genotypic and phenotypic heterogeneities emerge as complex and overlapped. In this study, we analyzed genotype-phenotype correlations with 74 Korean cases with NS or NSLD. Clinical diagnosis was NS in $56(76 \%)$ and NSLD in 18 (24\%) cases: 11 with cardiofaciocutaneous syndrome (CFC), 5 with Costello syndrome (CS), and 2 with LEOPARD syndrome (LS). Male predominance was noted in NS, but short stature and delayed development were more common in NSLD.
Mutations were identified in 38 cases with NS (67.9\%): 19 in PTPN11, 11 in SOS1, 4 in RAF1, 1 in BRAF, and 1 in KRAS. Nine cases out of 11 with CFC were confirmed to carry mutations, 4 in BRAF, 4 in SHOC2, and 1 in MEK1. All the 5 cases were with CS and 2 cases with LS harbor mutations in HRAS and PTPN11, respectively. Four cases with RAF1 mutation have hypertrophic cardiomyopathy. RAS and ERK activation assays were done to verify the functional perturbation after transient expression in HEK293 cells using mutant constructs. Patent ductus arteriosus was more common in cases with PTPN11 mutation than SOS1. Normal development and intelligence were more frequently observed in patients with SOS1 mutation than in other genes. There was no correlation in pulmonary stenosis, chest deformity, abnormal genitalia, lymphedema, hearing difficulty, or bleeding tendency. In conclusion, genotypephenotype overlap and correlations exist in NS and NSLD. Developmental delay is an important sign for the differential diagnosis of NS and NSLD. Further study is needed to discover novel gene(s) responsible for NS and NSLD with unknown genotypes.

\section{Oral Presentations (Concurrent Session 1)}

Thursday 18th November 2010

4:40PM-5:40PM

\section{The Mechanism of Insulin Resistance in Intrauterine Growth Retardation Rat}

\author{
Ruidan Zheng, Wujin Wang, and Xiao-ping Luo
}

Department of Pediatric, Tongji Hospital Affiliated to Tongji Medical College, Huazhong University of Science and Technology, Wuhan, China

Objective. As many studies show that adipose tissue plays a very important role in insulin resistance, we developed a catch-up growth intrauterine growth retardation (IUGRCG) model to investigate insulin resistance, glucose tolerance and the differentiation progress changes of adipose tissue in IUGR-CG rat. Methods. Using maternal nutrition-restriction during pregnancy to build IUGR model. 8 pups from the normal were fed as control group and 5 newborn IUGR pups as IUGR-CG group. Recording the body length and body weight at 0 week, 1week, 2 weeks, 3 weeks, 4weeks; fast serum glucose, insulin, triglyceride levels, and OGTT were examined in the 3rdw. Adipocytes and preadipocytes from the 4-week-old offspring's adipose tissue were cultured to test the multiplication capacity and the GLUT-4 expressing level. ShRNA of IRS- 1 and IRS-2 SOCS1/3 were designed and transferred into preadipocytes to examine C/EBP-a and PPAR-r expressing level. Result. Body weight and body length of IUGR-CG grow faster than in the control group and there is significant difference between the IUGR-CG and the control group at 4th (week $59.26 \pm 3.02 \mathrm{~g}, 74.69 \pm$ $5.81 \mathrm{~g}, P<.01)$. Fast and OGTT serum glucose and insulin level in IUGR-CG rats is higher than the control group $(P<.01)$ Fasting plasma triglyceride $(1.29 \pm 0.42 \mathrm{mmol} / \mathrm{L})$, 
insulin $(7.01 \pm 1.43 \mathrm{mU} / \mathrm{L})$, and insulin resistance idex $(1.32 \pm 0.41)$ of IUGR-CG rats were significantly higher than AGA rats $(0.75 \pm 0.40 \mathrm{mmol} / \mathrm{L}, 5.08 \pm 1.35 \mathrm{mU} / \mathrm{L}$, $0.83 \pm 0.20)$. There is no significant difference with the expression of GLUT4. However, preadipocytes of IUGRCG showed an increasing proliferation trend than AGA. RT-PCR and Western blot showed that IRS-1, IRS-2, and SOCS1/3 gene silencing downregulated the expression of C/EBP-a and PPAR-r in preadipocytes. Conclusion. Insulin resistance in IUGR-CG may be caused through the IRS1/2 and SOCS1/3 signal pathway. IRS-1, IRS-2, and SOCS1/3 expressing levels are upregulated in IUGR-CG rat, they induced the expression of C/EBP-a, and PPAR-r, which make IUGR-CG rat adipose tissue have a high proliferation and differentiation capacity. The heavily increased adipose tissue led to overweight, hypertriglyceridemia, insulin resistance, and impaired glucose tolerance. Catch-up growth in IUGR children may be a major risk factor or early manifestation for later obesity, type-2 diabetes, and cardiovascular diseases.

\section{Exercise Training Improves the Cardiovascular Function of Type 1 Diabetic Adolescents: An MRI Study}

\section{Silmara Gusso, ${ }^{1}$ James C. Baldi, ${ }^{2}$ Wayne Cutfield, ${ }^{1}$ and Paul Hofman ${ }^{1}$ \\ ${ }^{1}$ Liggins Institute, University of Auckland, Auckland, New Zealand \\ ${ }^{2}$ Department of Biological Sciences, Northern Arizona University, Flagstaff, USA}

The impact of exercise training on the left ventricular function (LVF) and femoral leg blood flow (FLBF) in type 1 diabetic (T1DM) adolescents is unknown. We describe the effects of 20 weeks of exercise training on LVF and FLBF at rest and during submaximal exercise (60\% of maximal heart rate) using magnetic resonance imaging in 38 T1DM (20 male) and 22 nondiabetic (ND) adolescents (10 male). Fifteen T1DM adolescents were recruited as nontraining controls (7 male). Aerobic capacity $\left(\mathrm{VO}_{2 \text { peak }}\right)$ was reduced in T1DM compared to controls $(44.6 \pm 5.7$ versus $48.3 \pm$ $6.6 \mathrm{ml} \mathrm{O} 2 / \mathrm{Kgffm} /$ minutes, resp., $P<.01)$. Baseline resting stroke volume (SV) indexed to fat free mass was smaller in the T1DM compared to ND adolescents (1.86 versus $2.05 \mathrm{~mL} / \mathrm{Kgffm}$, resp., $P<.01)$. Resting end-diastolic $(2.93$ versus $3.26 \mathrm{~mL} / \mathrm{Kgffm}$, resp., $P=.01$ ) and end-systolic volumes were smaller in the T1DM group (1.08 versus $1.20 \mathrm{~mL}$, resp., $P<.05)$. Untrained T1DM adolescents had a smaller SV during exercise compared to ND (1.88 versus $2.17 \mathrm{~mL}$, resp., $P<.01)$ and a smaller end-diastolic volume $(2.78$ versus $3.09 \mathrm{~mL}$, resp., $P<.01)$. Baseline FLBF was not different between groups at rest or during exercise; however the average area was smaller on the T1DM during exercise $(0.064$ versus 0.072 , resp., $P<.01)$. After training, both groups increased their aerobic capacity by an average $10 \%(P<.02)$ and the T1DM training group had significantly lower body fat percentage compared to nontraining controls $(25.7$ versus $28.4 \%$, resp., $P<.01)$.
Resting SV did not change with training $(P=.19)$. However, training increased exercise SV ( 1.9 versus $2.07 \mathrm{~mL}$ for T1DM and 2.17 versus $2.32 \mathrm{~mL}$ for ND, $P<.05)$. The increase in exercise SV was achieved by a decrease in end-systolic volumes on training groups ( 0.92 versus $0.80 \mathrm{~mL}$ for T1DM and 0.90 versus $0.84 \mathrm{~mL}$ for ND, $P<.05)$. Femoral leg blood flow did not change with training; however the average area increased in ND subjects during exercise $(0.072$ versus 0.074 , $P<.05)$. Glucose control was not improved with training ( 8.8 versus $8.6 \% \mathrm{~T} 1 \mathrm{DM}$ and 5.1 versus $5.1 \% \mathrm{ND}, P>.05$ ). In conclusion, T1DM adolescents had an impaired SV response to exercise which was improved following twenty weeks of exercise training. Thus, while diabetes is already limiting the cardiac function in adolescents, exercise training may help minimize these cardiac abnormalities.

\section{The Role of Phospholipase $A_{2} /$ Lysophosphatidylcholine Pathway in Hepatocyte Lipoapoptosis}

\section{Liu Lirui, Fu Junfen, Liang Li, and Shi Hongbo \\ Department of Endocrinology, the Children's Hospital of Zhejiang University School of Medicine, Hangzhou, China}

Background. Elevated serum free fatty acids (FFAs) and hepatocyte lipoapoptosis are important features of nonalcoholic fatty liver disease. Excess FFAs and the abnormal metabolites produced by FFAs are key reason for excessive hepatocyte lipoapoptosis. However, little is known about the underlying mechanism by which FFAs mediated lipoapoptosis. We presumed that the activation of phosphatidolipase $\mathrm{A}_{2} /$ lysophosphatidylcholine ( $\left.\mathrm{PLA}_{2} / \mathrm{LPC}\right)$ pathway may be a key signaling pathway in FFAs-induced hepatocyte lipoapoptosis, and phosphatidolipase $\mathrm{A}_{2}\left(\mathrm{PLA}_{2}\right)$ may be a key molecule in regulating hepatocyte lipoapoptosis. Objective. To investigate the role of $\mathrm{PLA}_{2} / \mathrm{LPC}$ pathway in FFAsinduced hepatocyte lipoapoptosis and its further molecular mechanism. Methods. Chang cells were incubated with different concentration of palmitic acid (PA), together with or without $\mathrm{PLA}_{2}$ inhibitors for $18 \mathrm{hs}$. Cell viability was assessed using the MTT assay. Cell apoptosis was determined by Hoechst33258 staining using fluorescence microscopy and AnnexinV-PI staining by ow cytometry. Intracellular lipids were measured by Nile red using digital fluorescent microscopy. PLA $\mathrm{P}_{2}$ expression was determined by Western blot and Q-PCR. Intracellular LPC content was measured by an enzymatic assay. Mitochondrial function was analyzed by detecting transmembrane potential and cytochrome $\mathrm{C}$ release. JNK expression and its inhibition by SP600125 and $\mathrm{PLA}_{2}$ inhibitors were measured by Western blot analysis of c-Jun phosphorylation. Result. The exposure of Chang cells to PA induced decreased cell viability and classical apoptosis in dose-dependent manner, and Chang cells treated with PA showed significant activation of $\mathrm{PLA}_{2}$, high levels of intracellular LPC content, mitochondrial transmembrane potential loss, cytochrome $\mathrm{C}$ release, and JNK phosphorylation. Pretreatment Chang cells with BEL, a specific inhibitor 
for iPLA 2 AACOCF3 a specific inhibitor for $\mathrm{CPLA}_{2}$ or $\mathrm{SPLA}_{2}$ IIA Inhibitor I, an inhibitor for sPLA $\mathrm{P}_{2}$ protected Chang cells' viability and significantly decreased PA-mediated lipoapoptosis $(P<.05)$. BEL and sPLA 2 -IIA Inhibitor I was also found to inhibit mitochondrial transmembrane potential loss, cytochrome $\mathrm{C}$ release and JNK phosphorylation. And pretreatment Chang cells with BEL or sPLA 2 -IIA Inhibitor I decreased intracellular LPC content, while AACOCF3 had no apparent effect on intracellular LPC content in PA-induced Chang cells, suggesting that the role of iPLA2 and sPLA2 is more important than CPLA2 in PA-induced LPC increase. Conclusion. These results showed that role of phospholipase $\mathrm{A}_{2} /$ lysophosphatidylcholine ( $\mathrm{PLA}_{2} / \mathrm{LPC}$ ) pathway appears to be an important event in the mechanism of PA-induced hepatocyte lipoapoptosis. It appears that the role of $\mathrm{PLA}_{2} / \mathrm{LPC}$ pathway in PA-induced hepatocyte lipoapoptosis depends on mitochondrion pathway of apoptosis and activation of JNK.

\section{The Relationship between the Metabolic Disturbances and the Content of Ghrelin in the Brain Tissue of Young Obese Rats with Metabolic Syndrome}

\author{
Zhang Shanshan, ${ }^{1}$ Liu Geli, ${ }^{1}$ Zhang Qiufeng, ${ }^{1}$ \\ Guo Jing, ${ }^{2}$ and Yang Qinyan ${ }^{1}$ \\ ${ }^{1}$ Tianjin Medical University General Hospital, \\ Tianjin, China \\ ${ }^{2}$ Tianjin Children Hospital, Tianjin, China
}

To approach the development and mechanism of metabolic syndrome (MS) in children and adolescents, we tried to establish the young SD rats model with metabolic syndrome by feeding the high fat and salt diet, which made the rats present the characters of obesity, disturbance of glucose metabolism, insulin resistance, disturbance of lipid metabolism, hypertension, and so forth. Ghrelin is a kind of peptide which can stimulate the release of growth hormone, promote the feeding behavior, regulate the energy metabolism, and so forth. In this research, we detected the concentration of ghrelin in the brain tissue of dietcontrolled rats and normal rats, compared the results, and evaluated if there was statistical significance among the groups. Meanwhile, we investigated the correlation between the concentration of ghrelin and the risk factors of the cardiovascular disease and observed the degree of their correlation, so as to discuss relationship between the change of ghrelin in brain tissue and the development of MS. We divided the 40 weaned rats (including 20 males and 20 females) into 3 groups randomly and, evenly, fed them with different diets including normal diet (NC group), and high fat diet (FC group), high fat and salt diet (FSC group). After 4 weeks, We took the biochemical tests (OGTT, insulin releasing test, and bloodlipids examination) and measured relative items (weight, waist circumference, blood pressure, etc.); we then got the brain tissue measured the concentration of ghrelin by the specific ELISA kit, and calculated the statistical significance of the difference among three groups. Finally, we investigated the correlation and its degree between the concentration of ghrelin and the risk factors of the cardiovascular disease. The results showed that high fat and salt diet could make 7 -week young rats present the characters of MS, which meant that the young SD rats model of metabolic syndrome was established successfully. At the same time, the concentration of ghrelin in the brain tissue of FSC group was lower while that of FC group was higher than that of the NC group, and the concentration of ghrelin was negatively correlated with the weight of the FSC and FC group. These results presented that ghrelin may regulate the metabolism on the CNS level and there might exist some feedback regulation of weight, blood glucose, and insulin.

\section{Oral Presentations (Concurrent Session 2)}

Thursday 18th November 2010

4:40PM-5:40PM

\section{Effects of Suckling Nutrition and Prepuberty Nutrition on Puberty Onset and the Expression of Hypothalamic Ghrelin in Female Rats}

\section{Lu-lu Cui and Pin Li}

Department of Endocriology, Shanghai Children' Hospital, Children's Hospital Affiliated to Shanghai Jiao-Tong University, Shanghai, China

Objective. To examine the adjustment effects of suckling nutrition and prepuberty nutrition on puberty onset and the expression of hypothalamic ghrelin by establishing different nutrition models of female rats. Methods. One-day-old female Sprague Dawley rats were randomly assigned to six groups. Each two suckling rats were fed by one lactation rat then received a high-fat diet (HFD) after being weaned at day 21 of age in Group A (sustained overnutrition group). Each two suckling rats were fed by one lactation rat then received a normal diet (NFD) after being weaned in Group $\mathrm{B}$ (Infancy overnutrition group). Each ten suckling rats were fed by one lactation rat and received HFD in Group C (Prepuberty overnutrition group). Each ten suckling rats were fed by one lactation rat and received NFD in Group D (Normal control group). Each twenty suckling rats were fed by one lactation rat and received NFD in Group E (Infancy malnutrition group). Each twenty suckling rats were fed by one lactation rat and received a low-fat diet (LFD) in Group F (sustained malnutrition group). To comparatively observe vaginal opening time, twenty rats in each group were randomly sacrificed when Group B showed complete vaginal opening and when Group D showed complete vaginal opening. Western blot was performed in all groups and the Ghrelin expression of hypothalamic was analyzed. Results. (1) The comparison of vaginal opening time of six groups is as follows: group A $29.20 \pm 1.96 \mathrm{~d}$, group B $29.50 \pm 1.73 \mathrm{~d}$, group C, $35.80 \pm 3.19 \mathrm{~d}$, group D $39.05 \pm 1.93 \mathrm{~d}$, group E $41.15 \pm 2.06 \mathrm{~d}$ and group F $42.70 \pm 1.69 \mathrm{~d}$. Group A and group $\mathrm{B}$ showed complete vaginal opening earlier than the other groups. It was delayed that the beginning of puberty onset of 
group $\mathrm{E}$ and group $\mathrm{F}(P<.05)$. (2) The expression of Ghrelin in hypothalamus of group A and group B was suppressed at 35 days, less than group $\mathrm{D}$, group $\mathrm{E}$, and group $\mathrm{F}(P<.05)$. While the expression of ghrelin in hypothalamus of group $\mathrm{A}$ and group B was raised up at 42 days, in other four groups, it was suppressed at the same time. Conclusions. This study indicated that nutrition effected the sexual development of female rats greatly, and the effects of suckling nutrition were much greater than prepuberty nutrition. Overnutrition was the negative feedback of the expression of ghrelin in hypothalamus. Malnutrition promoted the expression of ghrelin.

\section{The Influence of Downregulation of SOCS by RNAi on Glucose Transport of Intrauterine Growth Retardation Rats}

\section{Liao Lihong and Luo Xiaoping \\ Department of Pediatrics, Tongji Hospital, Huazhong \\ University of Science and Technology, Wuhan}

Objective. To measure the gene expression of insulin receptor substrate-1 (IRS-1) and suppressor of cellular signalling (SOCS-1 and SOCS-3) in insulin resistanceassociated intrauterine growth retardation (IUGR) rats and to evaluate the inhibition effect of small hairpin RNAs (shRNA) on the expression of SOCSs and insulinstimulated glucose transport in skeletal muscle cells of IUGR-born rats. Methods. An IUGR animal model was established by maternal food restriction in Sprague Dawley rats. Length, weight, body mass index (BMI), blood glucose, and plasma insulin were assessed at the age of 12 weeks. Levels of IRS-1, SOCS-1, and SOCS-3 mRNA and protein in the quadriceps femoris were measured by real-time quantitative PCR and Western blot. Constructed plasmids of small hairpin RNAs targeting SOCS1 and SOCS-3 were transfected into skeletal muscle cells via cationic liposome lipofectamine 2000, and Western blot and real-time quantitative PCR were used to verify the interference efficiency on SOCSs protein and mRNA expression levels. Insulin-stimulated glucose transport was also detected via immunofluorescence. Results. The insulinresistant IUGR rat model was successfully established. The length of IUGR-born rats was not significantly different; however, the weight, BMI, and the HOMA-IR of IUGRborn rats were higher than the control group. The outcomes of glucose tolerance test showed that the glucose levels of 30 and 60 minutes were higher in IUGR-born rats than that in the control group. The insulin level of 30 minutes was much higher in IUGR-born rats than those in the control group. IRS-1 expression was decreased and SOCS-1 and SOCS-3 were increased in the skeletal muscle of IUGR rats compared to the control group. The recombination plasmids PGPU6/GFP/Neo-SOCS-1shRNA and PGPU6/GFP/Neo-SOCS-3shRNA were successfully constructed and the shRNAs efficiently inhibited the expression of SOCS-1 and SOCS-3. Insulin-stimulated GLUT4 translocation was also dramatically elevated. Conclusions.
This data provides additional information on the mechanism of insulin resistance associated with IUGR. Downregulation of SOCS-1 and SOCS-3 ameliorates the capacity of glucose transport and provides a potential gene therapy approach to managing metabolic syndrome.

\section{Postterm Birth Is Associated with Obesity in Adolescent Males}

\section{Jacques Beltrand, ${ }^{1}$ Tanya Soboleva, ${ }^{2}$ Paul Shorten, ${ }^{2}$ Paul Hofman, ${ }^{1}$ Kerstin Albertsson-Wikland, ${ }^{3}$ Ze'ev Hochberg, ${ }^{4}$ and Wayne Cutfield ${ }^{1}$}

\author{
${ }^{1}$ The Liggins Institute, National Research Center for \\ Growth and Development, University Of Auckland, \\ New Zealand \\ ${ }^{2} \mathrm{Ag}$ 'research, Ruakura Research Centre, Hamilton, \\ New Zealand \\ ${ }^{3}$ Pediatric Growth Research Center, Sahlgrenska Academy, \\ Göteborg University, Sweden \\ ${ }^{4}$ Pediatric Endocrinology, Meyer Childrens' Hospital, \\ Rambam Medical Center and Rappaport Family Faculty of \\ Medicine and research Institute, Technion-Israel Institute \\ of Technology, Haifa, Israel
}

A deleterious prenatal and/or perinatal environment may lead to body composition and metabolic changes in childhood and later adult diseases. Arguably postterm (PT) infants have been exposed to an acute suboptimal nutrition during a prolonged gestation. The aim was to compare longitudinal growth in PT ( $\geq 42$ weeks of gestation) with term children (T) (37-41 weeks of gestation). 525 Swedish children, 250 boys/275 girls, with longitudinal records of weight and height from birth until 16 years (years) of age were analysed. 17 boys and 20 girls were PT. Growth velocity in height and weight was determined using a parametric mathematical model of children's growth from age 0 to 16 . At birth, in boys, weight (PT: $3.966 \pm 0.5 \mathrm{~kg}$, T: $3.640 \pm 0.5 \mathrm{~kg}, P=.01$ ) and length (PT: $52.2 \pm 1.8 \mathrm{~cm}$, $\mathrm{T}: 50.9 \pm 2 \mathrm{~cm}, P=.01)$ were greater in $\mathrm{PT}$ than in $\mathrm{T}$ whereas ponderal index was similar. In girls, only birth length was longer (T: $49.5 \pm 2 \mathrm{~cm}$, PT: $51.5 \pm 1.9 \mathrm{~cm}, P=$ .002). At 16 years, despite similar heights, the PT boys were markedly overweight (PT: $81.7 \pm 18.7 \mathrm{~kg}, \mathrm{~T}: 69.9 \pm 11.5 \mathrm{~kg}$, $P=.02$, BMI: PT: $25.4 \pm 5.5 \mathrm{~kg} / \mathrm{m}^{2}, \mathrm{~T}: 21.7 \pm 3.1 \mathrm{~kg} / \mathrm{m}^{2}$, $P=.002)$. There were no differences in height or weight at 16 years in girls. Using the mathematical model, similar height velocities were found from birth until 16 years in both groups and both genders. However, weight velocity was higher in PT boys during childhood (from 1.5 to 7 years of age, $P=.03$ ) and adolescence (from 11.5 to 15 years of age, $P=.03$ ). Furthermore, BMI started to be higher as early as 3 years of age in PT boys $(P=.03)$. The difference in BMI between PT and T boys increased until 7 years of age when it stabilized. It increased again during adolescence. BMI cues returned to parallel trajectories at the end of puberty in both groups, suggesting no further increase in BMI in PT boys. There was a trend towards an earlier puberty onset in 
PT boys (PT: $12.5 \pm 2.0$ years, T: $13.5 \pm 1.4$ years, $P=$ $.01)$. In summary, post term males have a greater BMI at 16 years. This likely reflects increased adiposity through a two-hit weight gain during childhood and adolescence. We speculate that nutritional reprogramming by late gestational placenta insufficiency increases the risk of later adult obesity and a metabolic syndrome phenotype in boys.

\section{The Changes of Molecules in Adipocyte Involved in Insulin Signal Transduction Pathway and Adipogenic Transcription Factors in Intrauterine Growth-Restricted Rats with Catch-Up Growth}

\author{
Jinzhi Gao and Xiaoping Luo \\ Tongji Hospital of Tongji Medical College of Huazhong \\ University of Science and Technology
}

Objective. There is increasing evidence that intrauterine growth-retardation (IUGR) and catch-up growth confer an increased risk of insulin resistance in later life. To observe the changes of molecules in adipocyte involved in insulin signal transduction pathway and adipogenic transcription factors to explore the possible mechanism of insulin resistance in rats with IUGR and catch-up growth. Research Design and Methods. We obtained timed pregnancies by vaginal smear, and then pregnant rats received ad libitum food (control) or were $30 \%$ food-restricted (IUGR). In lactation, the control groups were breast-fed by one mother rat per 10 pups, and the IUGR groups were breast-fed by one mother rat per 5 pups in order to make catch-up growth. Three-week-old pups were nursed and weaned to ad libitum diet. We cultured the adipocytes (around epididymis and kidney) from four-week-old rats and then identified adipocyte cells with oil red $\mathrm{O}$ staining. We compared mRNA levels of adipogenic transcription factors and insulin signal transduction pathway between IUGR pups and controls by quantitative real-time PCR. Knocked down IRS-1, IRS-2, and SOCS1/3 expression by shRNA in 3T3-L1 preadipocyte and then determined mRNA levels of adipogenic transcription factors by quantitative real-time PCR identified whether the preadipocytes differentiated into adipocytes or not by oil red $\mathrm{O}$ staining. Results. The IUGR pups showed upregulation of adipogenic transcription factors (PPARr, C/EBP-a) and SOCS1/3, and downregulation of IRS-1, IRS2, and PI3K. Knockdown of IRS-1, IRS-2, and SOCS1/3 expression by shRNA blocks preadipocytes differentiation and gene expression of PPARr and C/EBP-a. Conclusions. Results from this study may imply that IUGR and catchup growth block the insulin signal transduction pathway, in contrast to increasing adipogenic transcription factors. We speculate that catch-up growth promotes the ability of differentiation of preadipocyte, but the ability of glucose and lipid metabolism is permanently impaired in intrauterine with poor nutrition. The changes of molecules in adipocyte involved in insulin signal transduction pathway and adipogenic transcription factors could be the mechanism of insulin resistance in intrauterine growth-restricted rats with catch-up growth. The study provides new insights against insulin resistance.

\section{A Randomised Controlled Trial to Determine the Best Therapeutic Modality for Treatment of Nutritional Rickets}

Varun Aggarwal, ${ }^{1}$ Anju Seth, ${ }^{1}$ Raman K Marwaha, ${ }^{2}$ Satinder Aneja, ${ }^{1}$ and Pitamber Sonkar ${ }^{1}$

${ }^{1}$ Department of Pediatrics and Radiodiagnosis, Kalawati Saran Children's Hospital and Lady Hardinge Medical College, New Delhi-110001, India

${ }^{2}$ Department of Endocrinology, Thyroid Research Centre, Institute of Nuclear Medicine \& Allied Science (INMAS), New Delhi, India

Introduction. Rickets is usually thought to be due to vitamin $\mathrm{D}$ deficiency. However, recent studies have implicated dietary deficiency of calcium in its etiology. Information on relative efficacy of calcium, vitamin $\mathrm{D}$, or both together in treatment of rickets is limited. Objective. To study effect of treatment with calcium, vitamin $\mathrm{D}$, or a combination of these two on healing of nutritional rickets in young children. Design. Randomized controlled trial. Methods. We enrolled 67 cases of rickets in the age group of 6 months to 5 years. The baseline demographic (nutritional status, sun exposure (UV score), dietary calcium, and phytate intake) and biochemical data (serum calcium, inorganic phosphate, alkaline phosphatase, $25(\mathrm{OH}) \mathrm{D}_{3}$, and parathyroid hormone) were evaluated for all cases. They were randomly allocated to receive vitamin D (6 lakh IU single intramuscular injection), calcium (75 mg/kg elemental calcium daily), or a combination of the above two for a period of 12 weeks. Reassessment was done at 6 and 12 weeks for evidence of healing by a 10 -point radiological score and biochemical parameters. Results. Mean dietary intake of calcium in all cases was very low $(204.71 \pm 129.04 \mathrm{mg} /$ day $)$. Mean serum $25(\mathrm{OH})$ $\mathrm{D}_{3}$ level at baseline was in the range of mild deficiency $(15.94 \pm 12.43 \mathrm{ng} / \mathrm{ml})$. The combined end point of normal serum ALP and complete radiological healing at 12 weeks of treatment was seen in 50\% subjects on combination therapy as compared to $15.7 \%$ on vitamin D alone and $11.7 \%$ in group receiving calcium alone. Conclusions. Children with rickets had a low baseline serum vitamin D levels and low dietary calcium intake. The best therapeutic response was seen with a combination of both vitamin $\mathrm{D}$ and calcium, than either given alone. The treatment needs to continue beyond 12 weeks to ensure complete healing.

\section{Oral Presentations (Concurrent Session 1)}

Saturday 20th November 2010

9:00AM-10:30AM 


\section{Mild Noncompliance with Growth Hormone Therapy Is Associated with Poor Growth}

\author{
Wayne S Cutfield, ${ }^{1}$ José G B Derraik, ${ }^{1}$ \\ Alistair J Gunn, ${ }^{2}$ Kyle Reid, ${ }^{3}$ and Paul L Hofman ${ }^{1}$ \\ ${ }^{1}$ Liggins Institute, University of Auckland, Auckland, \\ New Zealand \\ ${ }^{2}$ Departments of Physiology and Paediatrics, \\ University of Auckland, Auckland, New Zealand \\ ${ }^{3}$ Pharmaceutical Management Agency of New Zealand \\ (PHARMAC), Wellington, New Zealand
}

Background. GH therapy requires daily injections over many years, and compliance can be difficult to sustain. As growth hormone $(\mathrm{GH})$ is expensive, noncompliance is likely to lead to suboptimal growth at considerable cost. Aims. (1) To assess the compliance rate of children and adolescents with $\mathrm{GH}$ treatment in New Zealand. (2) To determine the effects of GH treatment compliance on linear growth. Methods. This was a national survey of GH compliance, in which all children receiving government-funded $\mathrm{GH}$ for a four-month interval were included. Compliance was defined as $\geq 85 \%$ adherence (no more than one missed dose a week on average) to prescribed treatment. Compliance was determined based on two parameters: either the number of $\mathrm{GH}$ vials requested by the family or the number of empty GH vials returned. Results. 177 patients were receiving GH in the study period, aged $12.1 \pm 8.1$ years. The rate of returned vials, but not the number of vials requested, was positively associated with HVSDS right across the compliance range (see Figure 1). The rate of returned vials was therefore used for subsequent analyses. $66 \%$ of patients were noncompliant, and this outcome was not affected by sex, age, or clinical diagnosis. However, Maori ethnicity was associated with a lower rate of compliance.

Figure 1 (not shown). Compliance with GH therapy was compared to growth response over 6-8 months (height velocity SDS $)$. High $(n=30)$ is missing $\leq 1$ dose a week, medium $(n=51)$ is missing $>1$ and $<3$ doses/week, and low ( $n=29)$ is missing $\geq 3$ doses/week. Data are mean \pm SEM. $\left({ }^{*} P<.01\right),\left(*^{* *} P<.001\right)$ versus High. Conclusions. (1) Noncompliance with GH treatment is very common. (2) More than one missed GH dose on average each week adversely affects growth. (3) An objective assessment of compliance such as returned vials is much more reliable than compliance-based on parental or patient-based information. (4) Noncompliance should be considered in all patients with apparently suboptimal response to $\mathrm{GH}$ treatment.

\section{The Effect of Long-Acting Growth Hormone on Growth and Glucose Metabolism of Hypophysectomized Immature Rats}

\author{
Yingke Liu, Zhixin Zhang, Huimin Su, \\ Qiong Zhang, and Qiuling Zhao
}

China-Japan Friendship Hospital, Beijing, China
Objectives. The aim of this study is to observe and compare the growth-promoting effect of the long-acting growth hormone (LGH) and short-acting growth hormone (SGH) on hypophysectomized immature rats and to explore the changes of glucose metabolism after LGH intervention on these rats. Methods and Materials. Twenty-one-day-old SD rats were used to establish the growth hormone deficiency model with hypophysectomy. Two weeks after the operation 60 qualified young rats were selected and randomly divided equally into the model group (Group M), SGH intervention group (Group S), and LGH intervention group (Group L). Other 20 young rats were administered a sham surgery as the normal control group (Group N). All the drugs were injected subcutaneously in the neck of the rats at the same time of the day, $0.25 \mathrm{mg} / \mathrm{kg} \mathrm{SGH}$ daily in Group S, $1.4 \mathrm{mg} / \mathrm{kg}$ LGH on the first day and the same quantity of normal saline on other days of the week in Group L, the very same quantity of normal saline in Group $\mathrm{M}$ and Group $\mathrm{N}$ daily. The body weight, body length, and tail length were measured every other day after the administration. The random blood glucose level was examined on the 1, 3, 5, $8,10,13$, and 14 days at $8: 00 \mathrm{PM}$ and the fasting blood glucose level was detected on the 6,11 , and 15 days at 7:00 AM after 8 hours of fasting. Two weeks later 10 rats in each group received hyperinsulin-euglycemic clamping test while the other 10 were executed for the tibia length and epiphyseal plate width measurement. Results. At the end of each week, the body weight, tail length, and body length in Group L increased $(P<.05)$, compared with those in Group N and Group M, but was it not significantly different $(P>.05)$ from that in Group S. During each week, the body weight in Group L gained higher than that in Group $S$ in the first 4 days $(P<.05)$, but lower in the last 3 days $(P<.05)$. The length of tibia and width of epiphyseal plate in Group L increased $(P<.05)$ comparing with those in Group M and Group N, but similar with Group $S(P>.05)$. The random blood glucose level in Group L was lower than that in Group $\mathrm{N}$ in the 1st and 3rd day $(P<.05)$, but was not statistically different with those in Group M and Group S $(P>.05)$. There was no difference $(P>.05)$ of random blood glucose levels among the groups in the 5th, 8 th, and 10th days. But in the 13th and 14th day, this level in Group L was higher $(P<.05)$ than that in Group N and Group M, and again similar with Group $S(P>.05)$. The fasting glucose level of Group L was lower than that in Group N $(P<.05)$, but it was similar with those in Group S and Group M. The glucose infusion rate of the hyperinsulin-euglycemic clamping test in Group L was higher than that in Group N $(P<.05)$, but was not different from those in Group $\mathrm{M}$ and Group $\mathrm{S}$ $(P>.05)$. Conclusion. During the two weeks of intervention, we found that the LGH and SGH have similar effects on growth in the hypophysectomized immature rats, while the growth-promoting effect of LGH weakens gradually as time approaches the end of every week. Over time, the blood glucose levels are on the trend of rise, while the sensitivity of LGH and SGH to insulin did not significantly vary. 


\section{Final Height in Girls with Idiopathic Central Precocious Puberty Treated with Gonadotropin-Releasing Hormone Analogue and Recombinant Human Growth Hormone}

Hua-Mei Ma, Pi-Mei Zheng, Min-Lian Du, Zhe Su, Yan-Hong Li, Hong-Shan Chen, and Qiu-Li Chen

Department of Pediatrics, The First Affiliated Hospital, Sun Yat-Sen University, China

Objective. To evaluate the long-term final height outcome of combined treatment with gonadotropin-releasing hormone analogue (GnRHa) and recombinant human growth (rhGH) in girls with central precocious puberty. Methods. Out of forty-nine (49) girls with idiopathic CPP (treated with GnRHa at a dose of $60 \sim 80 \mu \mathrm{g} / \mathrm{kg}$ every 4 weeks for at least 6 months, whose height velocity (HV) fell below $4 \mathrm{~cm} /$ years and showed no improvement of predicted adult height in 6 months), 26 received (GH-combined group), in addition to $\mathrm{GnRHa}, \mathrm{GH}$ at a dose of $1 \mathrm{U} / \mathrm{kg} . \mathrm{W}$, sc, $6 \sim 7$ days weekly, for 11.4 $\pm 5.4(5 \sim 26)$ months; 23(control group) matched for $\mathrm{BA}$, chronological age, and duration of GnRHa treatment, who showed the same growth pattern but refused GH treatment, served to evaluate the efficacy of GH addition. Both groups discontinued treatment at a comparable BA (mean \pm SEM): $12.7 \pm 1.0$ in combined group versus $12.8 \pm$ 0.8 years in the control group. Results. At the conclusion of the study, all the girls had been followed up for $3.3 \pm$ 1.9 years and $3.2 \pm 0.9$ years in GH-combined group and control group, respectively, and had achieved adult height. Adult height was considered to be attained when the growth during the preceding year was less than $1 \mathrm{~cm}$, with a BA of over 15 years. During rhGH treatment, HV of the GHcombined girls increased significantly $((6.7 \pm 2.0) \mathrm{cm} / \mathrm{y}$ versus $<4 \mathrm{~cm} / \mathrm{y}$ baseline). GH-combined girls showed an adult height far higher than pretreatment PAH $((157.5 \pm 4.5) \mathrm{cm}$ versus $(148.1 \pm 4.6) \mathrm{cm}),(P<.01)$. Target height $(\mathrm{TH}$ : $(154.4 \pm 4.6) \mathrm{cm})$ was significantly exceeded. The control group reached an adult height also significantly higher than pretreatment PAH $((154.7 \pm 5.5) \mathrm{cm}$ versus $(150.3 \pm 6.0) \mathrm{cm}$, $P<.01$ ), while TH of $155.6 \pm 4.3 \mathrm{~cm}$ just reached but was not significantly exceeded. The gain in centimeters obtained, calculated between pretreatment $\mathrm{PAH}$ and final height, $9.4 \pm 4.9 \mathrm{~cm}$ in $\mathrm{GH}$-combined group was much more than $4.3 \pm 4.2 \mathrm{~cm}$ in control group $(P<.01)$. Furthermore, no severe side effects have been observed either on bone age progression or the gynecological followup in the $\mathrm{GH}$-combined group (in comparison with control group). Conclusion. rhGH may accelerate the linear growth and improve adult height of GnRHa-treated ICPP girls who show overgrowth reduction.

\section{Plasma C-Type Natriuretic Peptide in the Neonate Relation to Gestational Age and Linear Growth}

An-Ru Wang, Bao-Sheng Yu, Ye Shan, Lan-Ying Gao, and Yong-Quan Zhang
Department of Pediatric Endocrinology, the Second Affiliated Hospital of Nanjing Medical University, Nanjing 210003, China

Objective. C-type natriuretic (CNP) plays an essential role in endochondral bone growth. The relationship in plasma CNP, gestational age, and growth parameters in neonate were analyzed in order to reveal the effect of CNP on fetal and neonatal growth, whether related to IGF-1. Methods. 20 healthy newborns from obstetrical department and 30 sick newborns from neonatal department were enrolled. Growth parameters include height, weight, head circumference, and chest circumference. Plasma CNP and IGF-1 levels were determined by radioimmunoassay. Results. Cord plasma CNP of healthy newborns was inversely related to gestational age and birth weight. In neonatal ward, plasma CNP at the 1st day after birth was positive related to gestational age and all of growth parameters. Plasma CNP was correlated to growth velocity, and also as IGF-1. Plasma CNP at the 1st day was related to IGF-1. Conclusion. Relationship between plasma CNP and growth parameters at the 1st day suggested the essential role of CNP in fetal growth. Correlation of plasma CNP and growth velocity during the first 4 weeks of postnatal life indicated the effect of CNP on neonatal growth. CNP-effected growth may be concerned with IGF-1.

\section{Use Amino-Terminal Propeptide of C-Type Natriuretic Peptide to Evaluate the Effectiveness of Recombinant Human Growth Hormone Therapy in Idiopathic Short Stature and in Isolated Growth Hormone Deficiency}

\author{
Yuan Xiao, Wei Wang, and Defen Wang \\ Department of Paediatrics, Ruijin Hospital, School of \\ Medicine, Jiaotong University, Shanghai, China
}

Context. C-type natriuretic peptide (CNP) is a paracrine growth factor that plays an important role on regulating linear growth in bone plate. The level of amino-terminal propeptide of CNP (NTproCNP), splitting by proCNP, is easily measured in blood and reflects the rate of CNP biosynthesis. Objective. The aim of the study is to explore the value of amino-terminal propeptide of C-type natriuretic peptide (NTproCNP) in evaluating the effectiveness of therapy with recombinant human growth hormone (rhGH) in patients with idiopathic short stature (ISS) and isolated growth hormone deficiency (IGHD). Methods. Forty-eight prepuberty children (44 males, 4 females), with IGHD $(n=$ $25)$, mean aged (11.07 \pm 1.78 years $)$ and ISS $n=23$, mean aged (10.89 \pm 1.36 years), were randomly enrolled from Novmber 2007 to March 2009. Their heights were under 2 SDs before treatments and were examined for the serum concentration of insulin-like factor-I (IGF-I) and NTproCNP. They were all treated with rhGH (0.1$0.15 \mathrm{I} U / \mathrm{kg}$ every night) for at least one year. Serum IGF-I and NTproCNP levels were again measured at 6 months. After 
1-year injection, all patients were assessed some growthrelated clinical parameters, including annual growth velocity $(\mathrm{GV})$, height SD score (HtSDS), and gain height SD score $(\Delta \mathrm{HtSDS})$. Results. In GHD group, serum IGF-I SDS and NTproCNP levels at 6 months with GH therapy were significantly higher than before $(P<.001$, resp.), respond to the mean HtSDS increased at one year with GH treatment. It was showed the linear correlation between special bioparameter and promoting growth with $\mathrm{GH}$, the GV after therapy had a positive relationship with IGF-I SDS $(r=$ $0.407, P=.044)$ and the NTproCNP $(r=0.490, P=.013)$, respectively. The GH peak value had a positive relationship with IGF-I SDS and NTproCNP before rhGH therapy $(r=$ $0.558, P=.004, r=0.630, P=.001$, resp.); The IGF-I SDS and NTproCNP also had a positive relation $(r=$ $0.466, P=.019)$. In ISS group, the mean Ht SDS, serum IGF-I SDS, and NTproCNP levels at 6 months of therapy were also significantly higher than before $(P<.001)$. The linear correlation showed that the GV after therapy only had a positive relationship with the NTproCNP $(r=0.770$, $P=.001)$ and no relationship with $\Delta$ IGF-I SDS. The correlation between $\triangle$ IGF-I SDS and NTproCNP was not found. There were no significant differences in clinical and bio-parameters before and after therapy between ISS group and IGHD groups. Conclusions. Serum level of NTproCNP was a novel biomarker of growth, with increasing during growth-promoting treatment. It may have more clinical value in evaluation of children with short stature, predicting and monitoring the efficacy of rhGH therapy than IGF-I.

\section{Impact of Iron-Zinc Fortified Milk on Growth Velocity of Underweight Children, A Random- ized Controlled Trial}

\section{Annang Giri Moelyo, Endang Dewi Lestari, Leilani Lestarina, and Harsono Salimo}

\section{Child Health Department, Faculty of Medicine, Sebelas Maret University/Moewardi Hospital Surakarta, Indonesia}

Background. Zinc, as a micronutrient, is essential for somatic growth. It induces growth hormone $(\mathrm{GH})$ dimerization that make more stable than monomeric GH. GH is a major hormone for growth during childhood age. Underweight children need more, not only macronutrients but also micronutrients, to assure optimal growth. Objective. To evaluate the impact of iron-zinc fortified milk supplementation on growth velocity of underweight children. Methods. Randomized, double-blind, controlled trial, intention-totreat trial conducted in 10 elementary schools in Surakarta, Indonesia from July 2007 through February 2008. A total of 217 underweight children aged 7-9 years old were recruited in the study. Subjects who had chronic illnesses, severe anemia, thalassemia or cow's milk allergy were excluded. All participants were randomly assigned to receive either intervention milk (A) $(6.3 \mathrm{mg}$ of iron and $1.5 \mathrm{mg}$ of zinc) $(n=112)$ or standard milk (B) $(0.02 \mathrm{mg}$ of iron and $0.1 \mathrm{mg}$ of zinc) $(n=105)$. All subjects were anthropometrically measured (weight, height, sitting height) at 0 and 6 months of schedule, and then all plotted to CDC 2000 growth chart. Results. Study groups were comparable at baseline. Growth velocity of A group versus B group are 6.8 (SD 1.7) versus 6.3 (SD 2.2) $\mathrm{cm} /$ year $(P=.05)$. Boys' growth velocity of A versus $\mathrm{B}$ are 6.8 (SD 1.9) versus $6.0(\mathrm{SD} 1.9) \mathrm{cm} /$ year $(P=.026)$. Girls' growth velocity of A versus B are 6.8 (SD 1.5) versus $6.5(\mathrm{SD} 2.4) \mathrm{cm} /$ year $(P=.55)$. In short stature subjects, boys' growth velocity of A versus B are 6.6 (SD 2) versus $6.0(\mathrm{SD} 2) \mathrm{cm} /$ year $(P=.22)$ and girls' growth velocity of A versus B are 7.1 (SD 1.4) versus 6.8 (SD 2.5) cm/year $(P=.54)$. Conclusion. Zinc-iron fortified milk gives positive impact on growth velocity, especially boys.

\section{The Effect of Parental Origin of Intact $X$ Chromosome on Phenotypes in Korean Patients with Turner Syndrome}

\author{
Young Ah Lee, ${ }^{1}$ Jae Hyun Kim, ${ }^{2}$ Sun Hee Lee, ${ }^{3}$ \\ Ji Hyun Kim, ${ }^{4}$ Shin Mi Kim, ${ }^{1}$ Min Jae Kang, ${ }^{1}$ \\ Han Hyuk Lim, ${ }^{5}$ Hye Rim Chung, ${ }^{6}$ Shoong Ho Shin, ${ }^{1}$ \\ and Sei Won Yang ${ }^{1}$ \\ ${ }^{1}$ Seoul National University Children's Hospital, \\ Seoul National University College of Medicine \\ ${ }^{2}$ Ilsan Paik Hospital, Inje University College of Medicine \\ ${ }^{3}$ Pusan Paik Hospital, Inje University College of Medicine \\ ${ }^{4}$ Pohang St. Mary's Hospital \\ ${ }^{5}$ Chungnam National University College of Medicine \\ ${ }^{6}$ Seoul National University Bundang Hospital
}

The survival of $45, \mathrm{X}$ fetuses and various phenotypes in Turner syndrome (TS) can be associated with parental origin of intact $\mathrm{X}$ chromosome. The aim of this study was to evaluate the effect of parental origin of intact $\mathrm{X}$ on phenotypes including autoimmune thyroid disease (AITD), congenital cardiovascular or renal anomalies, and sensorineuronal hearing loss (SNHL) in TS patients with 45, X or X-isochromosome. A total of 49 TS patients with $45, \mathrm{X},(n=34)$ or $\mathrm{X}$-isochromosome, $(n=15,46$, $\mathrm{XiX}$ or $45, \mathrm{X} / 46, \mathrm{XiX})$ were enrolled between May, 2008 and July, 2009. TS patients with $45, \mathrm{X}$ without hidden $\mathrm{Y}$ fragment using PCR detecting 11 Y-chromosome sequences were included in this study. The parental origin of intact $\mathrm{X}$ chromosome was identified by comparing between patient and their parents using 9 highly polymorphic $\mathrm{X}$ chromosome microsatellites (DXS 1060, 8051, 1228, 1214, 986, 990, 1001, 1047, 8043). In 45, X patients, additional 3 microsatellites (DXS 1098, 993, 991) were analyzed if only one parent participated. Twenty-two (64.7\%) of 34 patients with 45 , $\mathrm{X}$ and $10(66.6 \%)$ of 15 patients with $\mathrm{X}$-isochromosome had a maternally derived intact X chromosome (Xmat). The proportion of Xmat in survived 45, X patients was high, $(P=.044)$. None of patients with 45 , X karyotype had hidden $\mathrm{X}$ fragments using 9 or $12 \mathrm{X}$-chromosome microsatellite marker analysis. Thirty-five $(72.9 \%)$ of 48 patients were diagnosed with AITD. The Xpat group had lower incidence of AITD than the Xmat group (50\% versus $84.3 \%, \mathrm{OR}=0.16, P=.022)$ irrespective of age, karyotype, regardless of GH therapy and family history of AITD. Only 
$9(25.7 \%)$ of 35 patients with AITD had a palpable goiter. The palpable goiter in TS patients with AITD was more commonly present in the Xpat group than in the Xmat group $(62.5 \%$ versus $14.8 \%, P=.015)$. Neither the presence of congenital cardiovascular anomalies nor congenital renal anomalies was affected by age, karyotype and parental origin of intact X chromosome. The presence of SNHL without conductive $\mathrm{HL}$ was associated with age (OR 1.33, $P=$ .016), but not karyotype and parental origin of intact $\mathrm{X}$, whether or not patients received GH therapy and duration of sex hormone deficiency since 11 years of age. This study may provide the evidence of an X-linked imprinting effect on AITD in TS patients. Although the survived 45, X patients may have higher incidence of maternally originated intact X chromosome, patients with Xmat may be more prone to AITD than those with Xpat. Further study to elucidate the mechanism of this imprinting effect on AITD is needed.

\section{Oral Presentations (Concurrent Session 2)}

Saturday 20th November 2010

9:00AM-10:30AM

\section{Kisspeptin Plasma Levels in Normal Female Pubertal Stages and in Girls with Idiopathic Central Precocious Puberty}

\section{Xiaoyu Ma, Jihong Ni, Yuejun Liu, Shaoke Chen, and Wei Wang \\ Department of Paediatrics, Ruijin Hospital, School of Medicine, Jiaotong University, Shanghai, China}

Objective. We investigate the pattern of the plasma kissprptin levels in various normal female pubertal Tanner stages and in the girls with idiopathic central precocious puberty(ICPP) or with the premature thelarche (PT) and evaluate the significance of detecting the plasma kisspeptin levels as a sensible criteria for differentiating the early diagnosis between the ICPP and PT. Methods. Plasma kisspeptin levels were determined in normal female pubertal girls in Tanner stage I-V $(n=16$ to 19$)$, girls with ICPP $(n=10)$ and PT $(n=12)$. Their levels were compared with their significance being analyzed statistically between various stages of normal puberty or between groups with ICPP and PT. The enzyme-linked immunosorbent assay (ELISA) was used to detect the plasma kisspeptin levels. Results. The levels of kisspeptin were significantly higher in the girls with ICPP than in those with PT:1.73 \pm $0.23 \mathrm{ng} / \mathrm{ml}$ versus $1.43 \pm 0.29 \mathrm{ng} / \mathrm{ml}(P<.05)$ and it was the highest among the normal pubertal females in Tanner stage $(1.73 \pm 0.22 \mathrm{ng} / \mathrm{ml})$ and significantly higher than all other stages $(P<.05)$, while in stage $(1.49 \pm 0.29 \mathrm{ng} / \mathrm{ml}$ and $1.50 \pm 0.36 \mathrm{ng} / \mathrm{ml})$ being higher than in stage $(1.23 \pm$ $0.30 \mathrm{ng} / \mathrm{ml})$ or $1.19 \pm 0.31 \mathrm{ng} / \mathrm{ml}(P<.01)$. Conclusions. Plasma Kisspeptin levels were the highest in Tanner stage during normal female pubertal development and significant difference revealed between the girls with ICPP and with PT.
The levels of plasma kisspeptin may be suspected as a useful criteria for distinguishing the ICPP and PT in their early stage.

\section{The Prevalence of Testicular Adrenal Rest Tumor and Its Associated Factors in Patients with Congenital Adrenal Hyperplasia Caused by 21-Hydroxylase Deficiency}

\author{
Min Jae Kang, Shin Mi Kim, Young Ah Lee, \\ Choong Ho Shin, and Sei Won Yang
}

\section{Department of Pediatrics, Seoul National University College of Medicine, Seoul, Korea}

Purpose. Congenital adrenal hyperplasia (CAH), caused by 21-hydroxylase deficiency, is a lifelong disorder, and issues on quality of life, sexual function and reproductive capacity should be considered in early adulthood. Particularly, testicular adrenal rest tumor (TART) in these patients has been known as a common and important cause of the infertility. Therefore, in the present study we aimed to observe the prevalence of TART, measured by testicular ultrasound, and to analyze its factors in patients with CAH. Patients and Methods. Testicular ultrasound was performed in 49 male patients (salt-wasting, 37; simple-virilizing, 12) with $\mathrm{CAH}$, whose ages were between 10.6 and 27.1 years. The prepubertal patients were excluded. We analyzed the past levels of serum 17-hydroxyprogesterone (17-OHP) from the time of ultrasound measurement tentatively to see if patients had been in undertreatment, which was calculated as a percentage of the number of measurement of serum 17-OHP levels higher than $10 \mathrm{ng} / \mathrm{mL}$ for the total number of measurement of serum 17-OHP during the follow-up period. Additionally, we tentatively divided 6 years of followup period just before ultrasound measurement (time 0 ) into three 2-year intervals and calculated the average concentration of serum 17-OHP of each interval (0-2 year average concentration (YAC), 2_4YAC and 4_6YAC). Results. TARTs were detected by testicular ultrasound in 32 of 50 patients (65.3\%). The median of maximal cross-sectional area of TART was $0.61 \mathrm{~cm}^{2}$ (range $0.01-4.95 \mathrm{~cm}^{2}$ ) and the median volume was $0.297 \mathrm{~cm}^{3}$ (range $0.001-8.181 \mathrm{~cm}^{3}$ ). The patients with TART showed lower corrected final adult height and higher 2_4YAC and body mass index than without TART, whereas other factors showed no differences. The maximal cross-sectional area of TART was positively correlated with undertreatment percentage $(r=0.444, P=.011), 2 \_4$ YAC $(r=0.355, P=.046)$ and (4_6YAC $r=0.518, P=.003)$. Conclusion. The positive rate of TART was $65.3 \%$ in male patients with $\mathrm{CAH}$, caused by 21-hydroxylase deficiency, whose occurrence and progression might be associated with the degree of disease control. Therefore, these results suggest that strict control of this disease is mandatory and regular check-ups with testicular ultrasound are recommended in male patients, regardless of the type of 21-hydroxylase deficiency. 


\section{Clinical Features in Patients with True Hermaphroditism (Ovotesticular Disorder of Sex Development)}

\author{
Shin Mi Kim, Min Kyung Kim, Young Ah Lee, \\ and Choong Ho Shin Won Yang
}

\section{Seoul National University College of Medicine, Korea}

Purpose. Ovotesticular disorder of sex development (OTDSD) was defined as the presence of both ovarian and testicular tissues in the same individual. The purpose of this study was to investigate clinical features at diagnosis and during followup in patients with OTDSD. Methods. A total of 21 patients with OTDSD following up 1980 and 2010 in Seoul National University Children's Hospital were included. We retrospectively investigated the medical records between at diagnosis and at thelast followup. Results. The karyotype was 46, XX (62\%, $n=13), 46$, $\mathrm{XY}(29 \%, n=6)$ and $46, \mathrm{XX} / 46, \mathrm{XY}(9 \%, n=2)$. The 40 gonads confirmed by pathology were ovotestis $(60 \%)$, ovary $(22.5 \%)$, and testis $(17.5 \%)$. The location of gonads was as follows: ovary (mostly in the left side, $88.9 \%$; in the abdomen or pelvis, $88.9 \%$ ), testis (mostly in the right, $85.7 \%$; in the scrotum, $57.1 \%$ ). The mean age at diagnosis was 3.4 years. The chief complaints at diagnosis were ambiguous genitalia (hypospadia, $n=10$; clitoromegaly, $n=6$, and inguinal hernia $(n=2)$ at initial visit and gyecomastia $(n=2)$ and inguinal mass $(n=1)$ during follow-up. Final gender assignment was male $(n=11)$ and female $(n=10)$; change in gender assignment with follow up in 4 of 21 patients was observed. Three patients (14.2\%) developed gonadoblastoma with late diagnosis of OTDSD in adolescence and adulthood; age at gonadectomy was 10 years, 12.9 years and 28 years of age, respectively. Conclusions. Early diagnosis of OTDSD is required to prevent the development of gonadoblastoma and to assign appropriate gender assignment. Both hypospadia and clitoromegaly were possible indications for diagnosis of OTDSD.

\section{Molecular Analysis of the DUOX2 Gene in Korean Patients with Congenital Hypothyroidism Caused by Dyshormonogenesis}

\author{
Hye Young Jin, ${ }^{1}$ Jin-Ho Choi, ${ }^{1}$ Sun-Hee Heo, ${ }^{2}$ \\ Bu-Won Jeon, ${ }^{2}$ and Han-Wook Yoo ${ }^{1,2}$ \\ ${ }^{1}$ Division of Pediatric Endocrinology and Metabolism, \\ Department of Pediatrics \\ ${ }^{2}$ Genome Research Center for Birth Defects and Genetic \\ Disorders, Asan Medical Center Children's Hospital, \\ University of Ulsan College of Medicine, Seoul, Korea
}

Purpose. Molecular studies have found that congenital hypothyroidism $(\mathrm{CH})$ is caused by genetic defects involved in dyshormonogenesis, such as thyroid peroxidase (TPO), thyroglobulin (TG), dual oxidase 2 (DUOX2), sodium iodide symporter (NIS), and pendrin (PDS). The DUOX2 enzyme activity is essential for generation of $\mathrm{H}_{2} \mathrm{O}_{2}$, the critical step in thyroid hormonogenesis. Previously, biallelic mutations in the DUOX2 gene result in severe and permanent $\mathrm{CH}$, while monoallelic mutations are associated with milder, transient $\mathrm{CH}$. This study aimed to investigate the correlation between clinical phenotype and molecular features of the DUOX2 gene defect. Patients and Methods. Thirty seven patients with $\mathrm{CH}$ with an ectopic thyroid gland were included in this study. They had been identified by newborn screening, and confirmed by serum thyroid function tests and thyroid scan. Patients with prematurity, down syndrome, and maternal autoimmune thyroid diseases were excluded. All coding exons of the DUOX2 gene and their intronic flanking sequences were amplified by PCR, and directly sequenced. Results. Twenty nine (78.3\%) out of 37 patients have permanent $\mathrm{CH}, 4$ patients $(10.8 \%)$ have transient $\mathrm{CH}$, while the remaining 4 patients were undetermined yet. Seven missense mutations (p.H678R, p.R885Q, p.L1067S, p.R1110Q, p.N43Y, p.G448R, and p.R1334W) were identified in $13(35.1 \%)$ out of 37 patients. Three mutations (N43Y, G448R, and R1334W) were novel. Ten out of 13 patients with DUOX2 mutations have permanent $\mathrm{CH}$, while the other 3 patients with DUOX2 mutations remain undetermined. Out of 10 permanent $\mathrm{CH}$ patients with DUOX2 mutation, 9 patients identified compound heterozygous or homozygous mutations, and one patient with permanent $\mathrm{CH}$ was heterozygous for p.R885Q. Three undetermined patients were heterozygous for p.L1067S, p.G488R, and p.R1334W. No mutation was found in four patients with transient $\mathrm{CH}$. Conclusions. This study identified seven mutations of the DUOX2 gene, including 3 novel mutations. Thirteen (35.1\%) out of 37 patients with $\mathrm{CH}$ caused by dyshormonogenesis have DUOX2 mutation, indicating that DUOX2 mutation is a common cause of $\mathrm{CH}$. Clinical phenotype of DUOX2 mutation can be variable, suggesting existence of alternative mechanism to replace DUOX2 or modifying factor to regulate DUOX2 expression. Functional analysis of novel mutations and long-term followup for undetermined patients should be needed.

\section{Association of a Single GDF5 Polymorphism with Human Weight and BMI in Chinese Population}

\section{Pei Zhou and Luo Feihong \\ Children's Hospital Of Fudan University}

Objective. Variation in the GDF5 gene has been reproducibly associated with osteoarthritis (OA) and human height in populations of UK, Finland and Sardinia. However, data from Asian is less conclusive. To identify the associations between GDF5 and human height, weight and BMI, we analyzed genome-wide association data from 1758 individuals of Chinese descent. Methods. We assessed the effect of GDF5 polymorphisms in a large population. A total of 862 males and 896 females had genotype data available. Data on height and weight were collected from participants at the time of 
blood collection. Results. Inconsistent with previous reports, no statistically significant association was detected between rs6060373 and height both in males and females $(P=0.749$ and 0.729 respectively). But we observed rs6060373 was associated with weight and BMI in males $(P=0.006$ and 0.006 respectively). The minor allele $\mathrm{G}$ was associated with heavier stature. No associations were observed in females. Conclusions. This population-based study shows that this GDF5 gene variant is associated with weight and BMI in Chinese males. And the selected SNP is not associated with height in Chinese people. To our knowledge this is the first demonstration of a compelling association between functional GDF5 SNP rs6060373 and human weight and $\mathrm{BMI}$ in the Chinese population.

\section{Urinary Gonadotropin Determinations during Gonadotropin-Releasing Hormone Analog Stimulation Testing in Children}

\author{
Zhuang-jian Xu, Xi-ping Wang, Ya-ping Ma, \\ Wen-jie Zhang, Xing-fu Pang, Qing Wang, \\ Shu-yan Xie, and Ting-ting Zhang
}

\section{Department of Pediatrics, The 4th Affiliated Hospital of Soochow University, Wuxi 214062, Jiangsu Province, China}

Objective. To assess the utility of timed urinary Gn detections as a substitute for serum gonadotropin determinations during GnRHa stimulation testing in children. Methods. Studies during $50 \mathrm{GnRHa}$ stimulation tests in children with a variety of growth or pubertal development disorders were performed. Five consecutive timed urine samples were collected, respectively, after triptorelin injection in all cases (the first was timed 1.5-hour urine, and the others were timed 1-hour urine, resp.). LH and FSH were measured by immunochemiluminometric assays (ICMAs). Results. (1)The peak urinary Gn (UGn) of the consecutive timed urine samples: the correlation coefficient between the quantities of peak urinary $\mathrm{LH}(\mathrm{ULH})$ and serum concentrations of peak LH(PLH) was 0.852 in first $3.5 \mathrm{~h}$ after triptorelin injection, for the peak ratio of ULH concentrations/creatinine (Cr) and serum PLH 0.774, and for (quantities of peak ULH)/(quantities of UFSHs), UFSHs were the quantities of urinary FSH in the same sample, and serum PLH/peak FSH (PFSH) 0.899. In the first $4.5 \mathrm{~h}$, the correlation coefficient between the quantities of peak ULH and serum PLH was 0.836 , for the peak ratio of ULH concentrations/Cr and serum PLH 0.774, for quantities of peak ULH/quantities of UFSHs and serum PLH/PFSH 0.894. The areas under the receiver operating characteristic (ROC)curves of quantities of peak ULH, peak ULH concentrations/Cr, (quantities of peak ULH)/(quantities of UFSHs) for the diagnoses of onset of HPGA were $0.960,0.923$, and 0.907 , respectively, in first $3.5 \mathrm{~h}$ after triptorelin injection, and in first $4.5 \mathrm{~h}$, were $0.952,0.928$ and 0.905 , respectively. Excluding the correlation coefficient between serum PLH and peak ULH concentrations/Cr (0.816) in first $2.5 \mathrm{~h}$, and between serum $\mathrm{PLH} / \mathrm{PFSH}$ and (quantities of peak ULH)/(quantities of
UFSHs) (0.904) in first $5.5 \mathrm{~h}$, the above other correlation coefficients as well as the areas under the ROC curves for quantities of peak ULH, for peak ULH concentrations/Cr, for (quantities of peak ULH)/(quantities of UFSHs) in first $2.5 \mathrm{~h}$ and in first $5.5 \mathrm{~h}$ were less than the highest in first $3.5 \mathrm{~h}$ and in first $4.5 \mathrm{~h}$, respectively. (2) UGn for different timed urine: the correlation coefficients between the quantities of ULH and serum PLH in the timed 2.5-hour, 3.5-hour, 4.5hour and 5.5-hour urine were $0.815,0.842,0.852$ and 0.814 , respectively, for the ULH concentrations/Cr and serum PLH $0.802,0.831,0.844$, and 0.814 , respectively. The areas under the ROC curves of the quantities of ULH for the diagnoses of onset of HPGA in the timed 2.5-hour, 3.5-hour, 4.5hour, and 5.5-hour urine were $0.913,0.944,0.958$, and 0.923 , respectively, for the ULH concentrations/Cr 0.911, $0.940,0.955$, and 0.919 , respectively. (3) Serum spontaneous Gn: the correlation coefficient between serum PLH and serum spontaneous LH (SLH) concentrations was 0.603, for serum PLH/PFSH and SLH/spontaneous FSH(SFSH) 0.566; the areas under the ROC curves of serum SLH and SLH/SFSH for the diagnoses of onset of HPGA were 0.792 and 0.675 , respectively. Conclusions. Our data suggest that urinary Gn determined by ICMA was better than serum spontaneous $\mathrm{Gn}$ dectections during GnRHa stimulation testing.

\section{Effects of Chinese Herbs for Nourshing Yin and Removing Fire on Expression of Gonadotropin-Releasing Hormone and Its Receptor, and Growth Hormone FSH LH of Female Rats with Environmental Endocrine Disruptors}

Qiao Lili, Sun Chunmei, Shen Weiqin, Fan Zhirong, and Zhang Xiaoli

Objective. To investigate the effects of Chinese Herbs for Nourishing Yin and Removing Fire on expression of Gonadotropin-releasing Hormone (GnRH) and GnRH receptor, Growth Hormone (GH), FSHLH mRNA and protein of female rats with mixture of octylphenol (OP) and bisphenol A(BPA). Methods. 21d immature female SD rats were divided into three groups: control group (cornoil), exposure group (OP100 mg/kg + BPA100 mg/kg), and treatment group (OP $100 \mathrm{mg} / \mathrm{kg}+\mathrm{BPA} 100 \mathrm{mg} / \mathrm{kg}+$ Chinese herbs), fed for 14 days, and the expression of GnRH and GnRH receptor, GHFSHLH mRNA and protein were determined by RT-PCR and immunohistochemical analysis. Results. The expression of GnRH and GnRH receptor, FSH, LH mRNA and protein significantly increased in exposed group as compared with control group. These indicators were reduced significantly after given Chinese herbs. Conclusion. Chinese herbs for Nourishing Yin and Removing Yang can inhibit the abnormal hyper function of hypothalamus-pituitary ovary in precocious puberty rats induced by EEDS and lower the responsibility of pituitary cell to GnRH. 


\section{Poster Abstracts}

Growth Hormone and IGF-1

\section{Long-Term Survey of 80 Patients with Childhood-Onset Primary Growth Hormone Deficiency}

\author{
Guoying Chang, Zhiya Dong, Wei Wang, \\ and Defen Wang
}

Department of Paediatrics, Ruijin Hospital,
School of Medicine, Jiaotong University, Shanghai, China

Objectives. The aim of the study was to evaluate the final adult height (FAH), lipid profile, sexual development and quality of life in individuals with childhood-onset growth hormone deficiency (CO-GHD), reassess the function of their GHIGF-1 axis in the transition from childhood to adulthood, and explore effective managements for different types of GHD in each period. Methods. 80 CO-GHD patients were divided into 2 groups isolated growth hormone deficiency (IGHD, $n=22$ ) and multiple pituitary hormone deficiencies (MPHD, $n=58$ ). There were 62 males with age $\geq 18$ years $(24.0 \pm 5.3$ years in average) and 18 females with age $\geq 16$ years $(22.2 \pm 4.8$ years in average $)$. We followed up clinical and biochemical parameters, education and occupation, rhGH and other hormones therapy in the past. FAH was defined as bone age $\geq 16$ years for boys and $\geq 14$ years for girls with the growth rate less than $1 \mathrm{~cm} /$ year. Results. The $\mathrm{rhGH}$ replacement therapy improved the $\mathrm{FAH}$ of patients with CO-GHD. FAH standard deviation scores (FAHSDs) were, respectively, $-1.19 \pm 0.9,-2.64 \pm 1.23$ in the $\mathrm{rhGH}$ replacement and not group $(t=4.61 P<.01)$. Long- or shortterm duration of rhGH treatment during their childhood show no statistical significant difference in the prevalence of occurrence of either hyperlipidemia (39.0\% versus $47.4 \%)$ or fatty liver disease $(26.8 \%$ versus $31.6 \%)$. Serum IGF-1 and IGFBP-3 remained at low levels; mean IGF-1 SDS and IGFBP-3 SDS were significantly higher in IGHD group than in MPHD group $(-1.43 \pm 0.31$ versus $-3.01 \pm 0.66$, and $-2.10 \pm 0.33$ versus $-3.17 \pm 0.19$ ), respectively. Patients with IGHD had normal sexual development, but $79.7 \%$ in MPHD group revealed sexual dysfunction with hypogonadotropic hypogonadism. Conclusions. The rhGH replacement therapy improves FAH of individuals with CO-GHD; serum IGF1 and IGFBP-3 levels remain low in the transition period; GHD patients have a high risk for metabolic abnormalities in the adulthood; the sexual development is not obviously impaired in IGHD group, while variable hypogonadotropic hypogonadism was apparent in all patients with MPHD.

\section{Long-Term Followup of Two Chinese Sibling Girls with Isolated Growth Hormone Deficiency Type IA}

\author{
Guoying Chang, Zhiya Dong, Wei Wang, \\ and Defen Wang
}

Department of Paediatrics, Ruijin Hospital, School of Medicine, Jiaotong University, Shanghai, China

Context. Isolated growth hormone deficiency type IA (IGHD IA) is an autosomal recessive disease caused by deletion of both growth hormone-1 alleles (GH1). We have followed two sibling girls with IGHD IA into the adulthood; the first case was genetically diagnosed, and received long-term recombinant human $\mathrm{GH}$ ( $\mathrm{rhGH}$ ) therapy. Objectives. The aim of the study was to examine the long-term efficacy and safety of rhGH therapy for two sisters with IGHD IA, track their sexual development and reproductive function, and reassess the quality of life in the adulthood. Subjects. Two Chinese sibling girls with documented IGHD IA were enrolled in this study. Genetic analysis showing a $6.7 \mathrm{~kb}$ spanning deletion of GH1 gene was identified before. They had been diagnosed at $11.7 \mathrm{yr}, 8.4 \mathrm{yr}$, respectively, with the height $89.3 \mathrm{~cm}(-7.8 \mathrm{SDs}), 80.6 \mathrm{~cm}(-8.8 \mathrm{SDs})$, and started rhGH substitution then. The duration of treatment was for 6.2 and 7.3 years. In the last visit, the ages were 31 and 29 years. Methods. We retrospectively reviewed the case notes of these 2 sisters, followed up their height, weight, blood pressure, and sexual development, measured fasting blood lipids, glucose, insulin, IGF-1, and IGFBP-3 and assessed educational and occupation status, and the growth and development of their children. Results. rhGH replacement had a significantly effect on growth with no evident side effects. The final adult height was $146.1 \mathrm{~cm}$ $(-2.6 \mathrm{SDs})$ and $153.0 \mathrm{~cm}(-1.3 \mathrm{SDs})$, they had normal spontaneous pubertal development and menarche at ages $15.2 \mathrm{yr}$ and $14.1 \mathrm{yr}$. Without rhGH treatment, pregnancy occurred spontaneously and was complicated by gestational diabetes mellitus (GDM). The growth and development of their children were normal. Levels of growth factors were still low in the adulthood, IGF- $1(46.6 \mathrm{ng} / \mathrm{ml}, 52.4 \mathrm{ng} / \mathrm{ml})$ and IGFBP$3(2460 \mathrm{ng} / \mathrm{ml}, 2430 \mathrm{ng} / \mathrm{ml})$. Though there was no metabolic syndrome happened, the fasting blood glucose rose with age $(6.6 \mathrm{mmol} / \mathrm{L})$. Conclusions. Long-term therapy with rhGH improves final adult height of individuals with IGHD IA; they can have normal sexual development and pregnancy, but there is a higher risk for metabolic abnormalities in the adulthood.

\section{Combined Treatment with Growth Hormone and Gonadotropin-Releasing Hormone Analogue in Adolescent Children with Idiopathic Short Stature}

Yuechun Teng, Wei Wang, Zhiya Dong, Jihong Ni, and Defen Wang

Department of Paediatrics, Ruijin Hospital,
School of Medicine, Jiaotong University, Shanghai, China

Background. Idiopathic short stature (ISS) characterized by heterogeneous genetic disorders show their adult height may have benefit by treatment with rhGH alone or combined with GnRHa when puberty developed, but still controversy on the variable effect on final adult height gain. Objectives. The 
aim of this study was to assess the efficacy of combination therapy with rhGH and GnRHa in Chinese children with ISS in early puberty. Methods. 30 children with ISS (15 males, 15 females), average age 11.9 years, with a height SD score of $-2 \mathrm{SD}$ or less in early puberty (Tanner stages $2-3$ ), were treated with GnRHa (80 $100 \mu \mathrm{g} / \mathrm{kg}$ every 28 days) and $\mathrm{rhGH}$ (0.1-0.15 IU/kg every night) for at least one year. 16 of them continued treatment for two years. Annual growth velocity $(\mathrm{GV})$, bone age (BA), height SD score for CA (HtSDSCA), height SD score for BA (HtSDSBA), and predicted adult height (PAH) SDS were assessed. Results. The following parameters all increased significantly; GV $2.3 \pm 1.2$ to $6.7 \pm$ $1.4 \mathrm{~cm} /$ years $(P<.05)$; HtSDSCA $-1.9 \pm 0.4$ to $-1.5 \pm 0.7$; HtSDSBA $-2 \pm 0.6$ to $-1.5 \pm 0.4$; PAHSDS $-1.8 \pm 0.7$ to $-1.1 \pm 0.7$ with $P$ value all <.001. BA/CA decreased from $1 \pm 0.1$ to $0.9 \pm 0.1(P<.05)$. By the end of the second year treatment, the GV slowed from $6.7 \pm 1.4$ to $5.5 \pm 1.8 \mathrm{~cm} /$ years $(P<.05)$, but was still much higher than pretreatment. HtSDSCA increased from $-1.5 \pm 0.7$ to $-1.3 \pm 0.5$, HtSDSBA from $-1.5 \pm 0.4$ to $-0.9 \pm 0.7$, and PAHSDS from $-1.1 \pm$ 0.7 to $-0.6 \pm 0.7$; these differences were also significant $(P<.001)$. Conclusion. This result demonstrated that this combined treatment regimen significantly improved the growth by delayed bone maturation and improved growth rate in pubertal children with ISS. Further followup is needed to verify the beneficial effects on final height gain.

\section{Comparative Evaluation of Short-Term Predictive Marker Response to Growth Hormone Treatment in Children with GHD Born Appropriate for Gestational Age or Small for Gestational Age: Results from a Phase IV Open-Label Study}

\author{
Wenli Lu, ${ }^{1}$ Wei Wang, ${ }^{1}$ Defeng Wang, ${ }^{1}$ \\ Shuixian Shen, ${ }^{2}$ Xue Fan Gu, ${ }^{3}$ Xiaoping Luo, ${ }^{4}$ \\ Chunxiu Gong, ${ }^{5}$ Minlian Du, ${ }^{6}$ Li Liang, ${ }^{7}$ \\ Runming Jin, ${ }^{8}$ and Qing Zhou ${ }^{9}$
}

${ }^{1}$ Ruijin Hospital, Jiaotong University, Shanghai, China ${ }^{2}$ Children Hospital, Fudan University, Shanghai, China

${ }^{3}$ Xinhua Hospital, Jiaotong University, Shanghai, China

${ }^{4}$ Tongji Hospital, Tongji Medical College of Huazhong

University of Science Technology, Wuhan, China

${ }^{5}$ Children Hospital, Beijin University, Beijin, China

${ }^{6}$ First Affiliated Hospital, Zhongshan University, Guangzhou, China

${ }^{7}$ Children Hospital, Zhejiang University, Hangzhou, China

${ }^{8}$ Xiehe Hospital, Wuhan, China

${ }^{9}$ MERCK-SERONO Pharmaceutical Ltd, Germany

Context. It is well known that GHD children born SGA who do not catch up are at significantly higher risk of short stature than children born AGA. SGA children with short stature have $\mathrm{GH}$ secretion abnormalities and lower levels of IGF-I. GH therapy can improve adult height in short stature children born SGA, but with large individual efficacy differences. There is no effective and practical prediction model for the efficacy of $\mathrm{GH}$ treatment in GHD children born AGA or SGA. Objective. To evaluate the short-term changes in GH-dependent biomarkers with GH treatment between GH-treatment-naïve Chinese GHD children born AGA or SGA. Methods. Data are from 8 centers participating in a phase IV open-label interventional Merck Serono study of recombinant human GH (r-hGH) treatment in GHD children. 213 GHD children, 183 AGA (134 males, 49 females; age $10.5 \pm 3.8$ years) and 30 SGA (24 males, 6 females; age $9.4 \pm 4.3$ years), were given daily $\mathrm{GH}$ for 4 weeks $(0.1 \mathrm{IU} / \mathrm{kg} /$ day $)$. All children were assessed pre- and posttherapy for changes in growth-related biomarkers (IGF-I and IGFBP3) and metabolic markers (fasting glucose, insulin, total cholesterol and HOMAIR). Results. The 213 GHD children had a baseline mean $\mathrm{GH}$ peak level of $4.5 \pm 3.1 \mathrm{ng} / \mathrm{mL}, 116$ of whom $(54.5 \%)$ had complete GHD $(<5 \mathrm{ng} / \mathrm{mL})$ and 97 (45.5\%) partial GHD $(<10 \mathrm{ng} / \mathrm{mL})$. Baseline serum IGF-I SDS and IGFBP3 SDS values were significantly lower than normal in all children (mean values -2.0 and -1.2 , resp.), but there were no significant differences between the two groups. Serum IGF-I levels increased by 1.30SD $(P<.0001)$, and IGFBP3 levels increased by $0.85 \mathrm{SD}(P<.0001)$ in all children from baseline to 1 month. No significant differences between the GHD-AGA group and the GHD-SGA group were found in any serum biomarker or metabolic marker changes at 1 month. Conclusions. These data show that one month of $\mathrm{r}-\mathrm{hGH}$ therapy significantly increased serum levels of growth-related biomarkers (IGF-I and IGFBP3) in GHD children, but there were no differential effects of therapy on growth-related biomarker and metabolic marker changes between GHD children born AGA or SGA. This suggests that the efficacy of $\mathrm{r}-\mathrm{hGH}$ treatment may be predicted by short-term changes in IGF-I and IGFBP3 in all GH-treated GHD children, including those born SGA.

\section{Association between Single Nucleotide Polymorphisms of Growth Hormone Receptor and the Risk of Idiopathic Short Stature}

\author{
Yi Yu, ${ }^{1}$ Wei Wang, ${ }^{2}$ Wei Huang, ${ }^{3}$ Ying Wang, ${ }^{3}$ \\ Zhiya Dong, ${ }^{2}$ Yuan $X i a o,{ }^{2}$ and Defen Wang ${ }^{2}$ \\ 1 Jiulong Hospital, Shanghai Jiaotong University, \\ Suzhou, China \\ ${ }^{2}$ Ruijin Hospital, Medical School, Shanghai Jiaotong \\ University, Shanghai, China \\ ${ }^{3}$ Chinese National Human Genome center Shanghai, China
}

Background. The linear growth of children is a complex process, involving both the internal (genetic) and external (environmental) factors. Children with idiopathic short stature (ISS) reveal no obvious pathologic findings, but some of them may present partial GH (growth hormone) insensitivity normal or higher serum GH levels and lower serum concentrations of insulin-like growth factor 1 (IGF-I) 
or GH-binding protein (GHBP). It has been reported that defects of GH receptor (GHR) gene as well as with their polymorphism could be associated with ISS. Aim. This study was to investigate the association of certain single nucleotide polymorphisms (SNPs) of the GHR with genetic susceptibility to ISS. Method. Genomic DNA was extracted from peripheral blood of children with ISS ( $n=$ 199) and of normal growth and development individuals as controls $(n=469)$. TagSNPs of GHR were selected from HapMap $\mathrm{CHB}$ subset and genotyping were conducted through the Illumina genotyping system. In single locus analysis, the risk of short stature was statistically analysed. Serum IGF-I and IGFBP-3 levels were measured in all patients. The association between the GHR gene polymorphisms with the height-related clinical traits was analysed. Result. 16 SNPs at GHR gene had been selected, but only 3 of which (rs4410646, rs10044169, and rs6182) showed their genotype frequency in children with ISS, and were significantly different from those of controls $(P<.05)$. However, there are no significant differences in their allele frequencies between the two groups. The genotypes of rs4410646 and rs6182 were respectively associated with the serum IGF1 levels $(P<.05)$. Conclusion. The results of our study supported the view that GHR gene polymorphisms play a role in the pathogenesis of ISS. Further research on the mechanism of these tagSNP may be needed.

\section{Analysis of Relationship between Growth Response during Growth Hormone Treatment in Short Children Born with Intrauterine Growth Retardation and Serum Level of Insulin-Like Growth Factor-1 and Insulin-Like Growth Factor Binding Protein-3}

\section{Zhaowen Chen and Zheng Zheng Zhan}

\section{Jin Shan Hospital of Fudan University, Shanghai 200540, China}

Objective. Our study is to evaluate the effect of growth hormone $(\mathrm{GH})$ treatment in short children born with IUGR and to explore the relationship between pretreatment serum level of insulin-like growth factor-1 (IGF-1), insulin-like growth factor binding protein-3 (IGFBP-3). Method. To collect 50 cases of short prepubertal children born with IUGR received growth hormone treatment at least one year in our hospital, comparing the differences of growth velocity before and after $\mathrm{GH}$ treatment and analyzing the relevance of pretreatment serum levels of IGF-1, IGFBP-3. According to their GH secretory status, they were divided into two subgroups, growth hormone deficiency (GHD) subgroup and non-GHD subgroup, comparing the differences of growth response between both groups. Result. (1) The growth velocity of short children born with IUGR significantly improved after GH treatment, $3.29400 \pm 0.65040 \mathrm{~cm} / \mathrm{y}$ versus $11.234 \pm 1.3338 \mathrm{~cm} /$ years. (2) Pretreatment serum level of
IGF-1, IGFBP-3 both have negative correlations with growth velocity after GH treatment, $(r=-0.589$ and -0.342 , resp.). (3) Comparing the differences of growth response after GH treatment, there was no significant differences between GHD subgroup and non-GHD subgroup. Conclusion. (1) Good growth response in growth velocity among short children born with IUGR shows that GH can effectively improve the height of short children born with IUGR. (2) The negative correlation of pre-treatment serum level of IGF-1, IGFBP-3 with growth velocity after GH treatment, indicates that the lower pre-treatment serum IGF-1, IGFBP-3 level, the better clinical effect of GH treatment. Pre-treatment serum level of IGF-1 and IGFBP-3 can be considered as makers predicting the growth response of GH treatment in short children born with IUGR. (3) The growth response between GHD subgroup and non-GHD subgroup shows no significant difference, which shows that the decision to treat a short IUGR child with GH therapy should not be based upon the $\mathrm{GH}$ response to a provocative test.

\section{The Study on the Change of Bone Maturation Speed and Final Adult Height of Children and Adolescents under Treatment by Growth Hormone}

\section{Kyung Rae Cho}

\section{Department of Pediatrics, Masan Samsung Hospital, Sungkyunkwan University School of Medicine, Masan, Korea}

Purpose. The purpose of this study was to evaluate the trend of the change of bone maturation speed and its effect on final adult height in patients of idiopathic short stature. Methods. Midparentral height, bone age, predicted adult height, final height, and the change of bone maturation speed were calculated in 37 people who were diagnosed with idiopathic short stature and treated with recombinant human growth hormone. We analysed these factors, then studied the correlation between the treatment period and the change of bone maturation speed, and the relation between the change of bone maturation speed and final adult height. Results. As time passes, the bone maturation speed was accelerated by the time passage in all patients. Predicted adult height increased until $21.2 \pm 6.3$ months after start of growth hormone treatment, thereafter decreased gradually. If midparental height is the target height, the final height attained was $3.3 \pm 2.7 \mathrm{~cm}$ more. Final height was $4.5 \pm 5.7 \mathrm{~cm}$ less than maximal predicted height, but $3.7 \pm 2.2 \mathrm{~cm}$ more than first predicted height. Conclusion. Even though predicted adult height will increase, then decrease and bone maturation speed will be accelerated in the people who were treated with recombinant human growth hormone, final height of patients was increased in comparison with midparental height and first predicted height. 


\section{Novel Compound Heterozygous Mutations for the IGF-1 Receptor Causing Severe Pre- and Postnatal Growth Failure and Dysmorphic Facial Features: A Family Study}

\author{
Yoon Hi Cho, ${ }^{1}$ Peng Fang, ${ }^{2}$ Michael A. Derr, ${ }^{2}$ \\ Vivian Hwa, ${ }^{2}$ Ron Rosenfeld, ${ }^{2}$ and Chris Cowell ${ }^{1}$ \\ ${ }^{1}$ Institute of Endocrinology and Diabetes, \\ The Children's Hospital at Westmead, Australia \\ ${ }^{2}$ Department of Pediatrics, Oregon Health \& Science \\ University, Portland, Oregon 97239-3098, USA
}

Aim. To describe the clinical outcomes of siblings with severe IGF-1 resistance. Method. Severe IGF-1 resistance was found in a male proband and younger sister. Molecular analysis of the IGF1R-gene in revealed novel compound heterozygous mutations, E121K(exon 2) and E234K(exon 3). Parents were heterozygous carriers (Table 1). Results. Patient 1 was born small for gestational age (SGA) at 36 wks to consanguineous Lebanese parents. At age 3.4 years, height was $72.2 \mathrm{~cm}$ (SDS -7.33) with height velocity $3.5 \mathrm{~cm} /$ years, weight $6.9 \mathrm{~kg}$ (SDS -9.31) and head circumference $42.5 \mathrm{~cm}$ (SDS -5.1). Bone age was 1.8 years. GH levels were nondeficient, IGF-1 persistently elevated and IGFBP-3 was within normal range. A one-year trial of GH therapy (14$28 \mathrm{IU} / \mathrm{m}^{2} / \mathrm{wk}$ ) did not result in catch-up growth. At age 14 years, antibody-negative insulin-dependent diabetes was diagnosed. He required learning support for intellectual disability. Final height at age 20.3 years was $133.2 \mathrm{~cm}$. Facial features include broad nasal bridge, synophrys, prominent epicanthii, low set ears, thin upper lip and small opalescent teeth. He has central adiposity and lumbar lordosis. Patient 2 was born SGA at 38 wks. Total pulmonary anomalous venous return, diagnosed postnatally, was repaired successfully. Severe growth failure ensued despite stable cardiac condition. IGF-1 was $1.71 \mathrm{U} / \mathrm{mL}$ (0.15-0.7). Similar facial dysmorphic features as Patient 1 were present. Other features included left convergent strabismus, fifth finger clinodactyly and café-au-lait lesions. At age 5 years, an abdominal Burkitt's lymphoma was diagnosed, resulting in fatal recurrence after six-months therapy. Conclusions. Patient 1 demonstrates a novel compound heterozygous mutation for the IGF-1 receptor causing severe pre- and postnatal growth failure and dysmorphic facial features. Possible associations arising from this family study include intellectual disability, nonimmune-mediated insulin-dependent diabetes mellitus and aggressive malignancy.

\section{Clinical and Functional Characteristics of the IGF1R Gene Mutation in Patients with Intrauterine Growth Retardation and Postnatal Catch-Up Growth Failure}

\author{
Jin-Ho Choi, ${ }^{1}$ Min-Ji Kang, ${ }^{2}$ Maria Hong, ${ }^{2}$ \\ Eul-Ju Seo, ${ }^{2}$ Jung-Young Park, ${ }^{2}$ Gu-Hwan Kim, ${ }^{2}$
}

Hye Young Jin, ${ }^{1}$ Beom-Hee Lee, ${ }^{2}$ Se-Min Lee, ${ }^{3}$ and Han-Wook Yoo ${ }^{1,2}$

\author{
${ }^{1}$ Division of Pediatric Endocrinology and Metabolism, \\ Department of Pediatrics \\ ${ }^{2}$ Medical Genetics Clinic and Laboratory, Asan Medical \\ Center Children's Hospital, University of Ulsan College of \\ Medicine, Seoul, Korea \\ ${ }^{3}$ Department of Pediatrics, Hanyang University Guri \\ Hospital, Seoul, Korea
}

Purpose. Around 10\% of infants born with intrauterine growth retardation (IUGR) remain short, and the causes of their growth deficits are often unclear. It is postulated that heterozygous IGF1R mutations underlie some cases of IUGR and postnatal growth failure. The objective of this study was to describe the clinical features of patients with IGF1R mutation and evaluate the molecular and functional characteristics of a novel IGF1R mutation. Methods. Three children with unexplained IUGR (birth weight $<-1.5$ SDS) and persistent short stature $(<-2.0$ SDS $)$ were included in this study. Auxological and endocrinological profiles including serum IGF-1 and IGFBP-3 levels, were measured. All coding regions including intron-exon boundaries of the IGF1R gene were PCR amplified and directly sequenced. To study the functional effect of the IGF1R gene mutation on IGF-1 signaling, IGF1R protein expression, IGF1R phosphorylation, and IGF-1-dependent Akt and Erk phosphorylation were measured by Western blotting using specific antibodies. Array comparative genome hybridization (CGH) was carried out to delineate the size and breakpoint of the cytogenetic abnormalities in a patient with dysmorphic features and multiple anomalies. Results. IGF1R gene analysis identified a novel heterozygous mutation of c.330del (p.Ala110fsX20) in exon 2 , resulting in a frame shift with premature termination of IGF1R protein. Her father and elder brother also carried the same mutation, indicating an autosomal dominant inheritance. The expression of $\operatorname{IGF} 1 \mathrm{R} \beta$ protein is reduced in patients compared with control cell lines. IGF-1-induced autophosphorylation of P-Tyr of IGF1R and phosphorylation of downstream signaling protein Akt were increased in a dose-dependent manner, less efficiently in a patient. Akt phosphorylation in patient cells needed significantly higher IGF-1 concentrations than control fibroblasts despite equal amounts of total Akt proteins. Array CGH of chromosome 15 of a patient with dysmorphic features and multiple anomalies identified a heterozygous deletion of 15 q26.2 to 15 qter. Conclusions. The novel heterozygous mutation resulted in reduced IGF1R expression and represents haploinsufficiency of the IGF1R gene. The novel heterozygous mutation in the $\alpha$-subunit of the IGF1R found in three members of one family leads to IUGR and postnatal growth. Fibroblasts from the c.330del (p.Ala110fsX20) propositus studied in vitro exhibited decreased IGF1R expression and IGF-1 resistance, as assessed by IGF1R phosphorylation and postreceptor signal transduction. The study suggests that IGF1R mutation should be considered in differential diagnosis in familial IUGR patients without catch-up growth. 
TABLE 1

\begin{tabular}{|c|c|c|c|c|}
\hline & Patient 1 & Patient 2 & Mother & Father \\
\hline Genotype & E121K/E234K & E121K/E234K & E121K & E234K \\
\hline Birthweight & SDS $-2.79(1.55 \mathrm{~kg})$ & SDS $-3.23(1.8 \mathrm{~kg})$ & - & - \\
\hline Birth length & SDS $-3.53(39 \mathrm{~cm})$ & SDS $-0.77(47 \mathrm{~cm})$ & - & - \\
\hline Clinical associations & $\begin{array}{l}\text { Antibody negative } \\
\text { diabetes mellitus }\end{array}$ & Burkitt's Lymphoma & Normal phenotype & Normal phenotype \\
\hline Height at $4-5$ years & SDS -6.4 & SDS -3.5 & & \\
\hline Final height & SDS $-5.91(133.2 \mathrm{~cm})$ & - & SDS $-1.16(155.6 \mathrm{~cm})$ & SDS $-1.44(166.5 \mathrm{~cm})$ \\
\hline IGF-1 (nmol/L) & 95.6 (RR 17.0-60.2) & - & $23.8($ RR 15.1-42.1) & $22.0($ RR 15.1-42.1) \\
\hline IGF-BP3 (mg/L) & 5.8 (RR 2.9-7.2) & - & 4.1 & 4.4 \\
\hline
\end{tabular}

\section{Factors Influencing Growth Response to Growth Hormone Therapy in Prepubertal Children with Chronic Kidney Disease}

\author{
Jae Hyun Kim, ${ }^{1,2}$ Young Ah Lee, ${ }^{2}$ Min Jae Kang, ${ }^{2}$ \\ Sei Won Yang, ${ }^{2}$ and Choong Ho Shin ${ }^{2}$ \\ ${ }^{1}$ Department of Pediatrics, Inje University, \\ Ilsan Paik Hospital, Goyang, Korea \\ ${ }^{2}$ Department of Pediatrics, College of Medicine, \\ Seoul National University, Seoul, Korea
}

Purpose. Chronic kidney disease (CKD) is a cause of short stature and recombinant human growth hormone (rhGH) has been used to treat growth failure in CKD patients. The aim of this study was to evaluate growth response of rhGH and factors affecting growth-promoting effect to rhGH therapy in growth-retarded prepubertal patients with CKD. Subjects and Methods. This study included 36 patients, who were prepubertal during the rhGH therapy. Inclusion criteria were as follows: patients on dialysis or conservatively treated patients with glomerular filtration rate of $<60 \mathrm{ml} /$ minutes per $1.73 \mathrm{~m}^{2}$, calculated by Schwartz equation; height of -2 standard deviation score (SDS) or height velocity (HV) of $<4 \mathrm{~cm} /$ years. All patients were treated with rhGH up to 3 years. Results. Among patients, 23 were on conservative treatment, and 13 were on dialysis at initiation of rhGH treatment. All patients were treated with $\mathrm{rhGH}$ at least for one year, 21 for two years, and 12 for three years. In conservatively treated patients, predicted adult height (PAH) SDS was $-1.6 \pm 0.7$ before $\mathrm{rhGH}$ treatment and $-0.9 \pm 0.7$ after rhGH treatment $(P<.001)$; in patients on dialysis, $\mathrm{PAH}$ SDS before and after rhGH treatment was $-1.8 \pm$ 1.6 and $-1.1 \pm 1.5$, respectively $(P=.073)$. However, $\mathrm{PAH}$ SDS of all subjects was increased significantly from $-1.7 \pm 1.1$ to $-1.0 \pm 1.1$ after rhGH treatment $(P<.001)$ (Table 2). Conclusion. In patients with CKD, rhGH treatment significantly increased height SDS, difference in height SDS, $\mathrm{HV}$ and PAH SDS in the short term. Long-term followup is to be warranted to elucidate the effect of rhGH to the final height.

\section{Long-Term Effects of Recombinant Human Growth Hormone Replacement Therapy on Adrenal Cortical Function in Children with Growth Hormone Deficiency}

\author{
Qian Wang and Guimei Li \\ Xiaojun Sun, Lijuan Zhang Provincial Hospital Affiliated \\ to Shandong University, Jinan 250021, China
}

Objective. To investigate the dynamic changes of cortisol (COR) and ACTH in growth hormone deficiency (GHD) children after long-term hormone replacement therapy (HRT). Methods. 50 growth hormone deficiency (GHD) children, whose clinical information were complete and had injected rhGH for more than 1 year were divided into Group I (GHD) and Group (MPHD). These patients were followed up in 3 monthly interval, serum levels of cortisol and adrenocorticotropic hormone (ACTH) were measured. Results. According to the data of 50 growth hormone deficiency (GHD) children, 60\% patient's levels of cortisol were decreased in group MPHD. Hydrocortisol replacement therapy was started before or at the same time of HRT. There was a significant negative linear regression between the replacement therapy dosage of hydrocortisol and pretreatment level of COR, giving a regression equation of dosage of hydrocortisol $(\mathrm{mg} \cdot \mathrm{m}-2 \cdot \mathrm{d}-1)=15.6-0.11 \times$ preCOR $(\mathrm{pmol} / \mathrm{L})(r=-0.88, F=35, P<.01)$. This also occured in isolated growth hormone deficiency (IGHD) patients, but the level of COR was maintained in normal range. The pretreatment levels of COR was positively correlated with the peak of growth hormone $(\mathrm{GH})$, serum level of insulin-like growth factor-1 (IGF-1) and height of anterior pituitary and negatively with pretreatment bone age difference (CA-BA). Conclusion. Central hypoadrenalism with symptoms or no symptoms can be observed in majority of MPHD children, which may be exacerbated during the process of rhGH therapy. Complete hormone replacement therapy and regular monitoring are necessary to maintain COR in lower limit for the best growth effect of rhGH therapy. Hydrocortisol is not needed in IGHD 
TABLE 2: Effects of 1 to 3 years of rhGH treatment on height SDS, height SDS difference and height velocity in prepubertal patients with CKD analyzed by repeated measures ANOVA.

\begin{tabular}{|c|c|c|c|c|c|}
\hline & \multirow{2}{*}{$n$} & \multirow[b]{2}{*}{ Baseline } & \multirow[b]{2}{*}{1 year } & \multicolumn{2}{|c|}{ rhGH treatment } \\
\hline & & & & 2 years & 3 years \\
\hline \multirow{3}{*}{ Height SDS } & 36 & $-2.7 \pm 0.9^{a}$ & $-2.1 \pm 1.0^{b}$ & & \\
\hline & 21 & $-2.6 \pm 0.7^{a}$ & $-2.0 \pm 0.9^{b}$ & $-1.6 \pm 1.0^{c}$ & \\
\hline & 12 & $-2.7 \pm 0.9^{\mathrm{a}}$ & $-2.1 \pm 1.0^{\mathrm{b}}$ & $-1.8 \pm 1.1^{\mathrm{c}}$ & $-1.6 \pm 1.1^{\mathrm{d}}$ \\
\hline \multirow{3}{*}{ Height SDS difference } & 36 & $-0.4 \pm 0.6^{\mathrm{a}}$ & $0.6 \pm 0.4^{\mathrm{b}}$ & & \\
\hline & 21 & $-0.2 \pm 0.5^{\mathrm{a}}$ & $0.7 \pm 0.4^{b}$ & $0.3 \pm 0.4^{\mathrm{c}}$ & \\
\hline & 12 & $-0.1 \pm 0.5^{\mathrm{a}}$ & $0.7 \pm 0.4^{\mathrm{b}}$ & $0.3 \pm 0.4^{\mathrm{c}}$ & $0.2 \pm 0.3^{\mathrm{a}, \mathrm{c}}$ \\
\hline \multirow{3}{*}{ Height Velocity (cm/years) } & 36 & $4.5 \pm 1.9^{\mathrm{a}}$ & $8.0 \pm 1.9^{b}$ & & \\
\hline & 21 & $4.8 \pm 2.0^{\mathrm{a}}$ & $8.7 \pm 1.9^{b}$ & $7.0 \pm 1.7^{\mathrm{c}}$ & \\
\hline & 12 & $4.6 \pm 2.3^{\mathrm{a}}$ & $8.3 \pm 1.5^{b}$ & $6.5 \pm 1.7^{c}$ & $6.0 \pm 1.3^{\mathrm{a}, \mathrm{c}}$ \\
\hline
\end{tabular}

Values not sharing superscript $a, b$, and c letters are significantly different from the values of other time points within each row.

\section{Correlation of Exon 4, 6, and 10 Single Nucleotide Polymorphisms of GHR Gene with Idiopathic Short Stature}

\section{Chen Linqi, ${ }^{1}$ Li Shiqin, ${ }^{1}$ Wang Ying, ${ }^{2}$ Xie Rongrong, ${ }^{1}$ Zhang Xuelan, ${ }^{1}$ and $\mathrm{Wu}_{\mathrm{H}}$ Haiying $^{1}$}

\author{
${ }^{1}$ Suzhou Children's Hospital, Suzhou University, \\ Jiangsu, China \\ ${ }^{2}$ Shang Hai Mankind Gene Group, Shanghai, China
}

Objective. The GHR gene polymorphisms have been found in idiopathic short stature. We studied the GHR (growth hormone receptor) gene exon 4, 6, and 10 SNPs and investigated the influence of these SNPs on serum IGF-I and IGFBP3, their influence on the axis of GHRH-GH-IGF-1, and height. Methods. 30 idiopathic short stature children who were in prepuberty were recruited in Soochow University Affiliated Children's Hospital between the September of 2008 and the July of 2009; average age was $(8.39 \pm 2.86$ years $)$. 45 controls (these controls were separated into 2 groups, one was normal group which contained 20 children whose heights were normal, the other contained 25 children whose heights were between the ISS and the normal) were collected in Soochow University Affiliated Children's Hospital at the same time, they were all in prepuberty. We measured the serum levels of IGF-1 and IGF-BP3 in case group and control group with chemiluminescence method, and contrast the case group and control group. We drew the DNA from the blood of the case group and control group children. Analysis of Polymerase Chain Reaction-Single Strand Conformation Polymorphism (PCR-SSCP) and DNA sequencing were both used to identify mutation in the GHR gene exons 4,6 , and 10 . We contrasted the polymorphism of the GHR gene exon 4,6 , and 10 between the control and case group. Using the $\chi^{2}$ test to detect the sex difference genotype and the allele frequency, using $t$-test and rank sum test to detect the age IGF-1 and IGF-BP3, using the OR to describe the risk ratio of the genotype and the allele frequency using the analysis of variance to detect the difference on IGF-1 and IGF-BP3 in serum among every genotype. All the data were processed using software of SAS 8.0. Result. There is no difference between the case group and control group in age and sex $(P>.05)$. The serum level of IGF-1 of ISS was not normally distributed, in contrast with the control group. Using $t$ test between the-1SD group and normal group, no differences were found $(P>.05)$; using rank sum test between the ISS group and normal group, there were differences $(P<.05)$; using rank sum test between the ISS group and-1SD group, there were differences $(P<.05)$; the serum level of IGF-BP3 of ISS and normal group are nonnormal distribution, and the-1SD group is normal distribution. Using rank sum test, there was no difference between the-1SD group and normal group $(P>.05)$, there was a difference between the ISS group and-1SD group and between the ISS group and the normal group $(P<.0001)$. We did not find any base change on exon 4. We found a SNP on exon 6, it is $6119 \mathrm{~A}>\mathrm{G}$ (G168G) and another SNP on exon 10, which was 10377 G $>\mathrm{T}(\mathrm{C} 422 \mathrm{~F})$. There was no difference in genotypic frequency and allele frequency on exon 6 119SNP among these groups $P>.05$; there was no difference in genotypic frequency and allele frequency on exon $10377 \mathrm{SNP}$ among these groups $P>$.05. On exon 6 119SNP here was no difference on serum level of IGF-1 among every genotype $P>.05$. On exon 10 377SNP here was no difference on serum level of IGF-1 among every genotype $P>.05$. On exon 6 119SNP there was no difference on serum level of IGF-BP3 among every genotype $P>.05$. On exon $10377 \mathrm{SNP}$ here was no difference on serum level of IGF-BP3 among every genotype $P>.05$. Conclusions. The serum level of IGF-1 can be the diagnosis index of prepubertal ISS. The serum level of IGF-BP3 can be the diagnosis index of prepubertal ISS. The SNP on GHR exon 6119 is not concerned with ISS. The SNP on GHR exon 6119 cannot influence the serum level of IGF-1 and IGF-BP3. The SNP on GHR exon 10377 is not concerned with ISS. The SNP on GHR exon 6377 cannot influence the serum level of IGF-1 and IGF-BP3. We should enlarge the number of the objects of study and engineer more primers so to make clear the reason of the decrease of IGF-1 and IGF-BP3. 


\section{User Acceptance of the Easypod Growth Hormone Autoinjection Device: Survey Results from 824 Children and Their Parents}

\author{
Fu-Sung Lo, ${ }^{1}$ Mauro Bozzola, ${ }^{2}$ Michel Colle, ${ }^{3}$ \\ Maria Halldin-Stenlid, ${ }^{4}$ Sylvain Larroque, ${ }^{5}$ \\ and Monia Zignani ${ }^{5}$
}

${ }^{1}$ Chang-Gung Memorial Hospital Linkou Wenhua 1st Road, Linkou, Taiwan

${ }^{2}$ Fondazione IRCCS San Matteo, University of Pavia, Pavia, Italy

${ }^{3} 25$ rue Boudet, Bordeaux, France

${ }^{4}$ Uppsala University, P.O. Box 256 SE-751 05 Uppsala, SWEDEN

${ }^{5}$ Merck Serono S.A.-Geneva, Germany

Background. Easypod is an electronic autoinjector for GH (Saizen, Merck Serono) administration. Aims. To assess patient acceptance of easypod and effect on adherence to recombinant human growth hormone (r-hGH) therapy through a large-scale survey study in 15 countries (Europe and Latin America). Methods. Study subjects were children using easypod for $\leq 3$ months and their parents. After institutional approvals were required and appropriate consent/assent procedures, the survey was administered by the nurse or investigator. Children and their parents were asked to rate specific easypod features on 5-point scales. Selfreported adherence to r-hGH therapy was also recorded, with those who reported missing $\leq 2$ injections per month (92\% of injections given) considered to be adherent to treatment. Adherence was also assessed by downloading the electronically recorded injection history from each easypod device. The dose-log diary was locked for patient/parent access during the 3-month study period. Results. A total of 824 children using easypod were surveyed (median (range) age: 11 (1-18) years; $56 \%$ were boys); $73 \%$ were naïve to $\mathrm{r}$-hGH therapy. $39 \%$ were responsible for their own injections (median (range) age 13 (5-18) years), parents were responsible in $53 \%$ of cases (median (range) age 9 (118 ) years) and $7 \%$ involved shared responsibility. Easypod was rated as easy/very easy to use by $\geq 80 \%$ (ease of use; preparation of the injection; changing cartridge and needle; holding and gripping the device). The injection was considered to be of short duration by $85 \%$, as silent/almost silent by $84 \%$ and as painless or less painful than expected by $62 \%$ and $58 \%$, respectively. In addition, $90 \%$ of children considered easypod comfortable/very comfortable to use and $92 \%$ wanted to continue to use it. For reported adherence, $52 \%$ of children missed $\geq 1$ injection over the 3 -month period, mainly due to forgetfulness ( $46 \%$ of reasons given). Overall, the electronically recorded adherence showed that $84 \%$ of children/parents were adherent (performed $\geq 92 \%$ injections). Adherence was significantly higher in treatmentnaïve children $(87 \%)$ than in those previously treated $(78 \%)$ $(P<.05)$. For $82 \%$ of children, reported and recorded adherences were the same. Conclusions. Children and parents reported that easypod was easy to use. Most adhered to therapy and wanted to continue using the device. These results demonstrate patient acceptance and clinical interest of an electronic injection device for daily recording and administration of $\mathrm{r}-\mathrm{hGH}$.

\section{Clinical Appearance on Child and Teenage Endorcrine Outpatients at Dr. Saiful Anwar General Hospital, Malang, Indonesia between 2005 and 2009}

\section{Harjoedi Adji Tjahjono \\ Department of Child Health, Brawijaya University Medical Faculty/Dr. Saiful Anwar General Hospital, Malang Indonesia}

Background. Many people and medical practitioners do not fully understand the importance of prompt and special treatment from pediatric endocrine specialists. Research Objective. To find as much relevant data as possible on pediatric and teenage endocrinology patients at Dr. Saiful Anwar General Hospital, Malang between 2005 and 2009. Methods. This was a retrospective, descriptive study using data obtained from medical records. Results. Over 5 years there were 576 endocrine patients, of which $346(60 \%)$ were males and $230(40 \%)$ were females. Ages ranged from first time treatment 224 patients (39\%) aged from $0-5$ years, 144 patients (25\%) aged 5-10 years, 150 patients (26\%) aged 10 15 years and $58(10 \%)$ aged over 15 years. Their diagnoses found: diabetes mellitus 144 (25\%), and Short stature 89 (15\%), Undescended testes 56 (10\%), Hypothyroid 54 (9\%), Obesity 54 (9\%), Micropenis 41 (7\%). Other ailments found: Hypothyroid, Disorders of Development, Pubertal Disorders, Hypospadia, Premature Telarche, Down's Syndrome, Gynecomastia, Turner's Syndrome, Achondroplasia, Osteogenesis Imperfecta, Tirotoxicosis, Marfan's Syndrome, Cushing's Syndrome and Osteoporosis. Conclusion. The most common disorders were Diabetes Mellitus 144 (125\%), Short stature $89(15 \%)$, and Undescended Testis 46 $(10 \%)$.

\section{X-Linked Recessive Combined Pituitary Hormone Deficiency Is Mapped to Xp22.1-Xp21.3 in a Chinese Family}

\section{Chun Lin Wang, Zhen Shen, Li Liang, Chao Chun Zou, Jun Fen Fu, and Guang Pin Dong}

Department of Endocrinology, Children's Hospital of Zhejiang University School of Medicine, Hangzhou 310003, China

Introduction. Combined pituitary hormone deficiency (CPHD) is associated with deficiencies of growth hormone (GH) and one or more of the other five anterior pituitary hormones. Genetic mutations have been identified in a modest proportion of patients with CPHD, including a number of autosomal genes (POU1F1, HESX1, PROP1) and X chromosome gene (SHOX, SOX3). Methods. we reported a three-generation family consisted of 16 subjects, 
including 5 affected males with hypothyrosis and $\mathrm{GH}$ deficiency. Genomic DNA was extracted from venous blood and 19 polymorphic DNA markers spread over the X chromosome were analyzed (DXS1060, DXS8051, DXS987, DXS1226, DXS1214, DXS1068, DXS993, DXS991, DXS986, DXS990, DXS1106, DXS1001, DXS1047, DXS1062, DXS1211, DXS984, DXS1205, DXS8091, DXS1073). Linkage analysis by MERLIN software and haplotyping was done. Results. All the affected males had the same haplotype on $\mathrm{X}$ chromosome and significant LOD scores were detected between DXS987 (Xp22.1-Xp21.3) and DXS 1226 (Xp22). Conclusion. CPHD with this family was the $\mathrm{X}$-linked recessive inherited disease and the pathogenical gene was mapped to Xp22.1-Xp21.3.

\section{PEG-Rhgh Therapy for Small or Gestational Age Rats and Effects on Their Metabolic Parameters}

\author{
Yanping $\mathrm{Xu},{ }^{1}$ Huang Ke, ${ }^{1}$ Li Liang, $^{1}$ Xiumin Wang, ${ }^{1}$ \\ Jin Pei, ${ }^{2}$ and Lizhong $\mathrm{Du}^{1}$ \\ ${ }^{1}$ Department of Endocrinology, The Children's Hospital of \\ Zhejiang University School of Medicine and Zhejiang Key \\ Laboratory for Diagnosis and Therapy of Neonatal Diseases \\ ${ }^{2}$ Department of Biochemistry and Molecular Biology, \\ Pharmacy School of Jilin University, Changchun 130021, \\ China
}

Aims. To establish a rat model of small for gestational age (SGA), to evaluate the effect of the PEG-rhGH in improving growth and to elucidate possible mechanisms for human metabolic syndrome in SGA subjects. Methods. Ten pregnant rats were randomly divided into two groups: (1) offspring from each undernourished rat whose birth weight less than -2SD as SGA model (2) offspring from each standard-nourished whose birth weight between mean \pm 1 SD as controls. Glycometabolism, blood pressure, glucose tolerance test and IGF-1 levels were analyzed. Insulin, IGF-1, PTP1B mRNAs were determined using real-time PCR. Results. Maternal undernutrition resulted in fetal growth retardation, influenced by decreased body weight at parturition in the offspring from SGA dams compared to controls. SGA rats at start of treatment were lighter and shorter than control animals. At the end of PEGrhGH treatment SGA animals had a greater body weight gain. The systolic blood pressure was higher in SGA treated with saline than that in appropriate for gestational age (AGA) group at the end of long-term follow up. At the same time points, SGA with saline-treated animals showed higher glucose levels at $60 \mathrm{~min}$ and $90 \mathrm{~min}$ as compared with AGA groups in glucose tolerance test. Conclusion. Follow-up study shows that after discontinuation of PEGrhGH treatment, fasting levels of glucose and insulin and IGF-I levels are comparable for PEG-rhGH treated and untreated in SGA rats. In addition, our data suggest that systolic BP is improved in PEG-rhGH-treated SGA rats.

\section{Change of Serum Fibroblast Growth Factor 21 (FG21) and Visfatin Levels during R-Hgh Treatment in Children with Growth Hormone Deficiency}

\author{
Li Yuchuan and Gong Chunxiu
}

Capital Medical University, Beijing Children's Hospital

Objective. To study the influence of recombinant growth hormone (rhGH) on FG21 Visfatin levels of children with growth hormone deficiency (GHD). Methods. Serum tryliceride (TG), total cholesterol (TC), insulin-like growth factor-1 (IGF-1), Fastin glucose, insulin, Adiponectin, Leptin FG21, and Visfatin levels were measured in 73 children, 57 boys, 16 girls, aged range 4.5-16.42 years old, average age $11.20 \pm 3.22$ years with GHD before and after rhGH replacement therapy 6 months. Results. After rhGH replacement therapy, the level of Adiponectin Leptin FG21 and Visfatin levels decreased and the level of FG21 was higher. And there was significant difference. Conclusions.rhGH replacement can improve the lipid profile in GHD children.

\section{The Value of Insulin and Arginine Provocation Test and the Effect of Recombinant Human Growth Hormone in Children with Growth Hormone Deficiency}

\author{
Liu Zheng Juan, Zhang Li, Bai Xue Mei, \\ and Wang Yu Chuan
}

\section{Department Of Pediatrics, Second Hospital Affiliated To Dalian Medical University}

Objective. Growth hormone deficiency (GHD) is one of the most important reasons for poor life quality. Clearly diagnosis is essential before treatment. This study evaluated the value of the insulin and arginine provocation test in children with short stature and anaylized the therapeutic effect of recombinant human growth hormone (r-hGH) in children with GH deficiency. Methods. GH provocation tests were performed with insulin and arginine in 65 short stature children aged 5-12 years, the GH concentrations were determined by RIA, and 35 children with peak $\mathrm{GH}$ concentration less than $10 \mathrm{ng} / \mathrm{ml}$ were defined as $\mathrm{GH}$ deficiency and treated with $\mathrm{r}-\mathrm{hGH}$ replacement therapy, with dosage of $0.1 \mathrm{IU} / \mathrm{Kg} \cdot \mathrm{d}$, injected subcutaneously every night, 27 children were treated for 12 months. Body height and body weight were monitored every month, the levels of serum T3, T4, TSH, IGF-1, and glucose were measured every 3 months, and the bone age was examined every 6 months. Results. The accuracy of insulin provocation test $(80 \%)$ was significantly higher compared with arginine provocation test $(66.7 \%)$, and the accuracy of both insulin and arginine provocation tests was $96.6 \%$. In insulin provocation test, nadir glucose level was less than $2.2 \mathrm{mmol} / \mathrm{liter}$, which occurred during the 15-30 minutes after insulin injection, and there was significant positive relationship 
between nadir glucose level and BMI. GH concentrations peaked at 30-60 minutes after insulin injection, and 6090 minutes after arginine injection. The GHD children with GH therapy for 12 months had increased in body height and height velocity. The height velocity after 3, 6, and 12 months therapy with $\mathrm{GH}$ was $13.8 \pm 2.4 \mathrm{~cm} /$ year, $14.7 \pm 2.4 \mathrm{~cm} /$ year, and $12.5 \pm 2.4 \mathrm{~cm} /$ year, respectively. Conclusion. The accuracy of insulin provocation test is better than arginine provocation test, and combination of drug provocation tests are more accurate than single drug provocation test. The GH replacement therapy for GHD children is effective, and the body height and height velocity are obviously increased, which is helpful for improving adult height.

\section{Study on the Deficiency of Shox Gene and the Correlation with Corresponing Phenotypes of Short Stature}

\author{
Yanli Cao, Min Zhu, Fang Xie, and Feng Xiong \\ Department of Pediatric Endocrinology, \\ Children's Hospital of Chongqing Medical University
}

Objective. To study the deficiency of short stature homeobox containing gene (SHOX) in short stature and the relationship between genotypes and its corresponding phenotypes. Methods. (1) 203 children with short stature, including 109 males, 94 females, were enrolled in Children' Hospital of Chongqing Medical University between July, 2008 and October, 2009. (2) 203 and 70 fresh blood samples were collected from patients and healthy children, respectively. (3) Radiographs of the left forearms and wrists of patients and healthy children were collected for the measurement of their skeletal features. Anthropometric measures were assessed, including height, sitting height, arm span, length of forearm and lower leg. (4) Genomic DNAs were extracted from fresh whole blood samples, then they were amplified with primer pairs designed to span 5 microsatllites of SHOX gene of patients and controls, as well as 5 SHOX exons of all patients. (5) Compared with controls, the SHOX deletions were identified by microsatellite analysis. (6) Directly sequencing exons of SHOX gene after exclusion of the patients without SHOX deletions, and then comparing the SHOX gene sequences of patients with of those of Gene Bank for the exploration of properly existed potential mutations. (7) Comparsion of the distinctive clinical manifestation of SHOX-deficient group, non-SHOX deficient participants, and the control group with normal height was made. Results. (1) 12 patients with SHOX gene mutations were found $(5.9 \%)$, including eleven deletions and a mutation of exon 2 were found in 203 patients with short stature. (2) Differences of physical signs between SHOX-deficient group and non-SHOX deficient participants of all patients, and the control group were tested by one-way ANOVA, such as physical height, and sitting height/height, arm span/height, forearm length/height, lower leg length/height. There were significant difference between SHOX-deficient group and
non-SHOX deficient participants, as well as between SHOX-deficient group and the control group. The sitting height/height of patients with SHOX deficiency increased, whereas their armspan/height, forearm length/height and lower leg length/height decreased. No differences in height were found between SHOX-deficient group and non-SHOX deficient groups $(P>$.05). (3) Radiographs of the left forearms and wrists of SHOX-deficient group indicated some mild skeletal deformities like Madelung deformity in patients with SHOX deficiency, but specific relationships between genotypes and bone deformities were not seen. Conclusions. (1) The study showed the SHOX deficient frequency of patients with short stature was $5.9 \%$. (2) The result of increased sitting height/physical height and decreased armspan/height of patients with SHOX deficiency suggested the ratio of increasing trunk and decreasing mesomelic limb; with decreased forearm length/height and lower leg length/height being characterized by the more severely shortened forearms and lower legs. (3) There were some mild and multiple skeletal deformities like Madelung deformity in patients with SHOX deficiency, which needed comprehensive analysis. (4) The height of short statures patients with SHOX deficiency markedly varies. Most of them had abnormal stature, although some were close to the normal height. This suggested that a high degree of phenotypic heterogeneity was associated with SHOX gene deficiency.

\section{Type 1 Diabetes}

\section{Weight and Body Fat Changes in Relation to Glucose Control before and 3 Months after Diabetes Camp in Children with Type 1 and Type 2 Diabetes Mellitus}

\author{
Azriyanti Anuar Zaini, ${ }^{1}$ Muhammad Yazid Jalaludin, ${ }^{1}$ \\ Chee Giap Tay, ${ }^{1}$ Suhaimi Hussain, ${ }^{2}$ \\ Muhammad Hadhrami Hussain, ${ }^{3}$ \\ Syamsul Rizal Abu Amin, ${ }^{4}$ Azril Syazwan Muhd Ali, ${ }^{4}$ \\ Zulkarnain Jaafar, ${ }^{4}$ Rosmini Tahir, ${ }^{1}$ Adam Bujang, ${ }^{5}$ \\ Jamaiyah H., ${ }^{5}$ and Fatimah Harun ${ }^{1}$ \\ ${ }^{1}$ Department of Pediatrics, University Malaya \\ ${ }^{2}$ Department of Pediatrics, University Sains Malaysia \\ 3 Ipoh General Hospital \\ ${ }^{4}$ Department of Sport Medicine Sciences, \\ University Malaya \\ ${ }^{5}$ Clinical Research Center, Kuala Lumpur Hospital
}

Introduction. Diabetes camp is meant to educate and improvise knowledge on diabetes, self-care, and blood sugar control in children with type 1 (T1DM) and type 2 diabetes mellitus (T2DM). Weight management, diet education, and exercise programme were also included to promote healthy lifestyle. Objective. To study the children's weight, body mass index (BMI), and body fat changes in relation to blood sugar control (HbAlc). Method. A 3-day diabetes camp was held on 3rd-5th December 2009. On arrival at 
camp site, weight, height and BMI of all children were documented. Body fat measurement was performed using body fat impedence analysis, and HbAlc was measured. Reassessment was performed 3 months after the camp. Result. A total of 36 children (30, 83.3\% T1DM) participated in this camp. There were $15(41.7 \%)$ Malays, $12(33.3 \%)$ Chinese and 9 (25\%) Indian participants. An equal number of both gender were seen. The mean age was 13.8 (3.0) years. Thirty-one $(86 \%)$ children returned for the 3-months post camp reassessment. Mean HbAlc pre- and postcamp were $9.5 \%$ and $9.2 \%$ respectively. There was no significant weight $(P=.265$ in T1DM and $P=.337$ in T2DM), body fat $(P=.554$ in T1DM and $P=.385$ in T2DM $)$ and HbAlc $(P=.349$ in T1DM, $P=.116$ in T2DM $)$ changes seen between pre- and post-camp measurement. However, there was a significant change in BMI $(P=$ $.002)$ in all children. No significant correlation was found between HbAlc level and the children's weight, BMI, and body fat. Conclusion. This study showed that BMI of all children was significantly reduced and sustained 3 months after the camp which may suggest that they understood the importance of weight management and adapting healthy lifestyle. However, this study could not demonstrate a better blood sugar control with the improvement of BMI.

\section{The Frequency and Characteristics of Hyperglycemic Hyperosmolar Syndrome in Children and Adolescents with Diabetes Mellitus}

\section{Cao Bingyan, Gong chunxiu, Dong Qian, and Huang Hui}

\section{Beijing Children's Hospital affiliated of Capital Medical University}

Objectives. Hyperglycemic hyperosmolar syndrome (HHS) is thought to be a rare entity in the pediatric population, associated with significant mortality based on case reports in the literature. This study will report the frequency and characteristics of HHS in children and adolescents with diabetes mellitus in our hospital. Methods. Patients diagnosed with type 1 and type 2 diabetes mellitus at Beijing Children's Hospital over a period from 1995 to 2009 were screened retrospectively for any laboratory evidence of previous episodes of HHS. The standard diagnostic criteria were refered from reference. Results. The records of all patients with type 1 diabetes mellitus (T1DM) and type 2 diabetes mellitus (T2DM) diagnosed over a 15 years period were reviewed ( $n=1034,906$ were T1DM, 128 were T2DM). Eleven patients were found to have HHS by diagnostic criteria (five males, mean age at presentation 11.1 years, age range $6.3 \sim 17.2 \mathrm{yr}$ ), so frequency of HHS was $1.06 \%$. The frequency of HHS in T2DM was a little higher than in T1DM (1.56\% versus $0.99 \%) .10$ of 11 cases combined with DKA simultaneously. Only 1 of 11 individuals had $\mathrm{PH}$ $\geq 7.25$, and 2 of 11 individuals had CO2 $\geq 15 \mathrm{mmol} / \mathrm{L}$. All eleven cases had evidence of ketonuria on presentation.
Mean effective serum osmolality was $334.18 \pm 8.82 \mathrm{mOSM} / \mathrm{L}$, mean blood glucose was $53.19 \pm 16.48 \mathrm{mmol} / \mathrm{L}$, mean $\mathrm{PH}$ was $7.13 \pm 1.16$, mean corrected Natrium was $153.21 \pm$ $6.37 \mathrm{mmol} / \mathrm{L}$. HHS or combined with DKA was the clinical presentation at diagnosis of new onset diabetes for all eleven children. Abdominal pain and vomiting were common in these HHS patients $(60 \%-70 \%), 80 \%-90 \%$ had typical symptoms of diabetes (polyuria and polydipsia); lethargy, weakness, confusion were also common. Highcarbohydrate drinks (2 cases with T1DM), carbohydrate fluid infusion ( 3 cases with T1DM), insidious onset (1 case with T2DM), and drinking less (1 case with Down syndrome) precipitate the development of HHS in DM. The presentation was more severe in T2DM than in T1DM, and the mortality also higher in T2DM. Only one T2DM girl died within the first 24 hours. She had the highest corrected Natrium $(164.5 \mathrm{mmol} / \mathrm{L})$ and effective serum osmolality (351.4 mOSM/L) and developed multisystem organ failure. The mortality rate of HHS in this group is $9.09 \%(1 / 11)$. No obvious neural system sequelae had been seen in survivors. Conclusions. HHS is a rare entity in children. Its frequency in diabetes is much less than DKA but more severe and higher mortality. The frequency of HHS is higher in T2DM than in T1DM in children. It is easily confounded by the presence of acidosis due to the severe hypovolemia, with a similar presentation with DKA. Corrected sodium and effective serum osmolality may correlate with the prognosis. Fluid composition, tonicity and infusion velocity should be carefully calculated during treatment. Increasing awareness of HHS, the morbidity and mortality related to HHS can be prevented.

\section{A Case Report of Neonatal Diabetes Mellitus with Confirmed Novel ABCC8 Mutation and Improvement in Glycemic Control after Switch from Insulin to Oral Sulphonylurea Therapy}

\author{
Hung Ching Ngar and Lee Ching Yin

\section{Department of Paediatrics and Adolescent Medicine,} \\ Princess Margaret Hospital, Hong Kong
}

We report a 4-year-old girl who presented with diabetic ketoacidosis during an admission for fever and upper respiratory tract symptoms at eight months old. She was treated with insulin infusion and then put on regular subcutaneous insulin. She had history of hyperglycemia at 4 months of age when she attended a hospital in Mainland China for fever and diaper rash but mother did not seek further medical advice afterwards. The haemoglobin Alc level at diagnosis was $14.7 \%$ and remained about $8 \%$ when she was treated with subcutaneous insulin at 0.2 unit per kilogram per day. Neonatal form of diabetes mellitus was suspected. Molecular study confirmed a novel ABCC8 mutation in the sulphonylurea receptor. The treatment was then changed to oral sulphonylurea. The glycemic control improved and the girl has maintained haemoglobin A1c level of 5 to $6 \%$. 


\section{Lower Sex Hormone-Binding Globulin Levels Are Associated with Cardiac Autonomic Dysfunction in Girls with Type 1 Diabetes}

Yoon Hi Cho, ${ }^{1}$ Maria Craig, ${ }^{1,2,3}$ Shubha Srinivasan, ${ }^{1}$ Paul Benitez-Aguirre, ${ }^{1}$ and Kim Donaghue ${ }^{1,2}$

${ }^{1}$ Institute of Endocrinology and Diabetes, The Children's Hospital at Westmead, Australia

${ }^{2}$ Discipline of Paediatrics and Child Health, University of Sydney, Australia

${ }^{3}$ School of Women's and Children's Health, University of New South Wales, Australia

Aim. To examine the association between androgen levels/insulin resistance and reduced heart rate variability (HRV), a marker of cardiac autonomic dysfunction, in girls with type 1 diabetes (T1DM). Methods. We report pilot data from 53 T1DM girls, median age 15 years (range 10.5$17.9)$ and duration 8 years (1.8-16.0). Heart rate variability (HRV), defined as oscillation in time intervals between consecutive heart beats (measuring R-R intervals $=\mathrm{NN}$ on a 20 minute ECG-recording), were compared with normal values derived from healthy school-based female controls. Statistical analyses used included SDNN(standard deviation NN), an estimate of overall HRV, and root mean squared difference of $\mathrm{NN}$ (RMSSD), estimate of short-term HRV. Spectral methods included low-frequency (LF)/high-frequency(HF) ratio, the balance between sympathetic/parasympathetic controls. Associations between SHBG/androgen levels and clinical parameters and HRV (log-values for nonparametric data) were analysed by linear regression. Results. Tanner staging was T4-5 $(n=35), \mathrm{T} 2-3(n=7)$, and T1 $(n=1)$. Lower SHBG was associated with longer diabetes duration, higher BMI-SDS, total daily dose insulin/weight (TDD), but not associated with age, waist:hip ratio, or HbAlc. Higher testosterone levels were not associated with the above variables. Lower SDNN was associated with higher HbAlc, longer duration, older age, and lower SHBG. Lower RMSSD was associated with longer duration and lower SHBG, whilst higher LF/HF-ratio was associated with lower SHBG but not other variables (Table 3). Conclusions. In adolescents with T1DM, early cardiac autonomic dysfunction was associated with low SHBG, a surrogate marker of insulin resistance. SHBG may help to identify those at greatest risk of early cardiac autonomic dysfunction, a known risk factor for diabetic morbidity and mortality.

\section{Incidence of Type 1 Diabetes in Children under 15 Years of Age in the Auckland Region (New Zealand) Over a 20-Year Period}

Wayne Cutfield, ${ }^{1,2}$ José Derraik, ${ }^{1}$ Alistair Gunn, ${ }^{3}$ Paul Hofman, ${ }^{1,2}$ and Craig Jefferies ${ }^{1,2}$

${ }^{1}$ Liggins Institute, University of Auckland, Auckland, New Zealand

${ }^{2}$ Paediatric Diabetes Service, Greenlane Clinical Centre, Starship Children's Health, Auckland, New Zealand
${ }^{3}$ Department of Physiology, University of Auckland, Auckland, New Zealand

Introduction. A steady increase in the incidence of type 1 diabetes mellitus (T1D) is being observed worldwide, and overseas studies suggest that this increase has been particularly pronounced among very young children $(<5$ years old). Objective. This study aimed to evaluate the changes in the incidence of T1D in children under 15 years of age in the Auckland region (New Zealand) over a 20-year period. Methods. We performed a retrospective review of all patients under 15 years of age that were diagnosed with T1D over a 20-year period (1990-2009) in the Auckland region, New Zealand. Results. There were a total of 885 new cases of T1D recorded in the region. There has been a marked increase in the incidence of T1D ( $n$ per 100,000 people) in children in the Auckland region (+0.68/years; $\left.r_{2}=0.70 ; P<.001\right)$, so that T1D incidence has risen from 7.01 in 1990 to $19.82 / 100,000$ in 2009. However, the rise in T1D incidence was not even among all age groups, being progressively greater with increasing age bands: $0-4$ years $\left(+0.37 /\right.$ years; $\left.r_{2}=0.40 ; P<.01 ; n=222\right), 5-9$ years $\left(+0.62 /\right.$ years; $\left.r_{2}=0.35 ; P<.01 ; n=327\right)$, and $10-14$ years $\left(+1.04 /\right.$ years; $\left.r_{2}=0.58 ; P<.001 ; n=336\right)$. Conclusions. There has been a considerable increase in the incidence of T1D diabetes in children in the Auckland region, with a nearly 3 -fold increase over the 20 -year period covered by this study. However, unlike other studies, the greatest increase in T1D incidence has occurred in older children, particularly among those between 10 and 15 years of age.

\section{0-Year Review of Type 1 Diabetes Mellitus in Children and the Clinical Value of Interleukin-10 in Diabetic Ketoacidosis of Childhood Type 1 Diabetes Mellitus}

\author{
Yang-li Dai, Jun-fen Fu, Guan-ping Dong, \\ and Li Liang

\section{Department of Endocrinology, The Affiliated Children's Hospital, College of Medicine, Zhejiang University, Hangzhou 310003, China}

Objective. To review the incident status of childhood type 1 diabetes mellitus hospitalized in Children's Hospital of Zhejiang University School of Medicine from 1999 to 2009 and to explore the clinical value of IL-10 in diabetic ketoacidosis of childhood type 1 diabetes mellitus. Methods. Two hundred and sixty three cases of childhood type 1 diabetes mellitus in Children's Hospital of Zhejiang University School of Medicine were collected from January 1999 to February 2009. The clinical characteristics were analysed retrospectively. Serum lipid was assessed in forty eight diabetic children and twenty four healthy children who were enrolled from December 2nd, 2006 to February 6th, 2009. Three subgroups were selected according to the diagnostic criteria: type 1 diabetes mellitus with ketoacidosis, type 1 diabetes mellitus without ketoacidosis, and control group. Serum lipid, cytokines were compared in these three 
TABLE 3: Comparison of HRV and clinical parameters stratified for SHBG in T1DM compared to controls.

\begin{tabular}{|c|c|c|c|}
\hline & Lowest tertile SHBG in T1DM girls & Higher tertiles SHBG in T1DM girls & Control \\
\hline \multicolumn{4}{|l|}{ HRV measures } \\
\hline Mean HR (bpm) & $79^{* *}$ & $71^{*}$ & 65 \\
\hline $\operatorname{SDNN}(\mathrm{ms})$ & 63 & 81 & 91 \\
\hline RMSSD (ms) & $53^{* *}$ & 80 & 98 \\
\hline $\mathrm{LF} / \mathrm{HF}$ ratio & 1.16 & 0.76 & 0.88 \\
\hline \multicolumn{4}{|l|}{ Clinical parameters } \\
\hline Age (years) & 14.8 & $14.3^{*}$ & 16.8 \\
\hline Duration (years) & $9.6^{* *}$ & 7.5 & \\
\hline HbAlc (\%) & 8.8 & 8.3 & \\
\hline BMI-SDS & $1.23^{* *}$ & 0.80 & \\
\hline Insulin dose ( $\mathrm{u} / \mathrm{kg} /$ day $)$ & $1.21^{* *}$ & 0.95 & \\
\hline \multicolumn{4}{|l|}{ Androgen profile } \\
\hline Free androgen index (\%) & $9^{* *}$ & 4.6 & \\
\hline Testosterone (nmol/L) & 2.5 & 2.6 & \\
\hline Androstenedione (nmol/L) & 7.8 & 6.4 & \\
\hline DHEAS (nmol/L) & 2.8 & 1.4 & \\
\hline
\end{tabular}

${ }^{* *} P<.05$ lowest versus higher SHBG tertiles in T1DM girls

$* P<.05$ higher SHBG tertiles in T1DM versus non-diabetic girls.

groups. Results. The result shows female polarization. The ratio of male to female was $0.78: 1$. The peak incident age was at the age of 6 to $11.9(55.1 \%, 145 / 263)$. There were eighty six $(32.7 \%, 86 / 263)$ children with ketoacidosis when they were first admitted to our hospital. The levels of serum lipid, blood glucose and HbAlc were significantly higher in diabetic children with ketoacidosis than those without ketoacidosis $(P<.05)$, and the increased levels of blood glucose, serum lipid and HbA1c were risk factors of diabetic ketoacidosis by Logistic analysis. The comparisons of 72 children with cytokines assessed:The level of serum IL-10 which was significantly higher in diabetic children with ketoacidosis than those without ketoacidosis $(P<.01)$, whereas, no differences were detected in IL-2, IL4, IL-6, TNF$\alpha$, IFN- $\gamma$. and Serum IL-2, IL-4, IL-6, IL-10, TNF-alfa, and IFN-gama were significantly higher in diabetic children than those in healthy children $(P<.01)$. Conclusion. The most common acute complications of type 1 diabetes mellitus ketoacidosis, and disorders of glucose and lipid metabolism in diabetic children with ketoacidosis were the risk factors of ketoacidosis. The level of serum IL-10 was significantly higher in diabetic children with ketoacidosis, and IL-10 may be a sensitive index of diabetic ketoacidosis in childhood type 1 diabetes mellitus.

\section{Abnormal Cardiovascular Function in Adolescents with Type 2 Diabetes May Impair Exercise Capacity}

Teresa Pinto, ${ }^{1}$ Silmara Gusso, ${ }^{1}$ Tim Hornung, ${ }^{2}$ Chris Baldi, ${ }^{1}$ Wayne Cutfield, ${ }^{3}$ and Paul Hofman ${ }^{1}$
${ }^{1}$ Liggins Institute, University of Auckland, Auckland, New Zealand

${ }^{2}$ Department of Paediatric Cardiology, Auckland District Health Board, Auckland, New Zealand

${ }^{3}$ Department of Biological Sciences, Northern Arizona University, Flagstaff, Arizona, USA

Exercise is part of standard recommendations in the management of Type 2 Diabetes (T2D); however maximal exercise capacity may be limited in both adults and adolescents with T2D irrespective of deconditioning and may be secondary to central (cardiac) and/or peripheral (vascular) abnormalities. Aims. (1) determine exercise capacity (VO2 peak) in a group of adolescents with T2D compared to an obese, nonT2D group and a non-obese control group, (2) to assess cardiac function in these groups with the use of cardiac magnetic resonance imaging (MRI) at rest and during sub-maximal exercise, and (3) to assess femoral artery flow (FAF) at rest and immediately after-exercise as a marker of peripheral vascular function. Methods. 13 subjects with T2D, 27 overweight or obese subjects and 19 nondiabetic, nonobese controls, aged 12-20 years were recruited. All subjects performed an incremental exercise test on a cycle ergometer. Body composition was determined using dual-energy x-ray absorptiometry (DEXA). Cardiac and femoral flow MRI images were taken at rest and during or immediately after sub-maximal exercise using a cycle ergometer designed for use in the MRI. Results. Maximum heart rate (HR) achieved was significantly higher in the control group than in the two other groups despite comparable maximum workloads. There was no difference 
in cardiac output (CO) indexed for fat-free mass (FFM) between groups at rest however, during exercise CO/FFM was significantly lower in the T2D group compared to the obese, and control group $(P<.01, P<.001$ resp.). SV increased by $11.1 \%$ in the control group, $5.98 \%$ in the obese group and $0.76 \%$ in the T2D group with exercise. End-diastolic volume (EDV)/FFM was significantly lower in the T2D both at rest and with exercise. During exercise, EDV decreased by $2.1 \%$ in the control group, $0.9 \%$ in the obese group and $6.1 \%$ in the T2D group. End-systolic volume (ESV)/FFM was comparable between groups at rest but was significantly lower in the T2D group with exercise. The average FAF/minute and the net forward volume both indexed for FFM were significantly lower in the T2D group after exercise compared to the other 2 groups. Conclusion. Independent of obesity, T2D negatively affects both central and peripheral vascular function during exercise in adolescents. Central causes appear to be secondary to impaired filling and possible diastolic dysfunction, while peripheral blood flow to exercising muscles may also be impaired. Both may hinder one's ability to engage in physical activity.

\section{Glycaemic Control, Frequency of Self-Monitoring Blood Glucose and Diabetes Knowledge in Type 1 Diabetes Children before and after Attending Diabetes Camp}

Muhammad Yazid Jalaludin, ${ }^{1}$ Azriyanti Anuar Zaini, ${ }^{1}$ Suhaimi Hussain, ${ }^{2}$ Hadhrami Muhammad Hadhrami, ${ }^{3}$ Chee Giap Tay, ${ }^{1}$ Rosmini Tahir, ${ }^{1}$ Adam Bujang, ${ }^{4}$ Jamaiyah Haniff, ${ }^{4}$ and Fatimah Harun ${ }^{1}$

${ }^{1}$ University Malaya

${ }^{2}$ Department of Pediatrics, University Sains Malaysia

${ }^{3}$ Department of Pediatrics, Ipoh General Hospital

${ }^{4}$ Clinical Research Center, Kuala Lumpur Hospital

Background. Diabetes camp provides the opportunity to increase patient's self-confidence and improves. the ability to manage diabetes on their own. In small groups, participants can learn about correct injection technique, testing and monitoring, diet, physiology, and other issues relating to diabetes. Aim. To evaluate the effectiveness of diabetes camp in improving glycaemic control and self-monitoring blood glucose (SMBG) and to provide diabetes knowledge to children with type 1 diabetes mellitus (T1DM). Method. Children with diabetes mellitus aged 8-18 years were invited to participate in the annual diabetes camp held on 3rd to 5th December 2009. On arrival at the camp site, diabetic profiles were documented, HbAlc were measured, blood glucometer was downloaded to assess the frequency of SMBG, and a standard diabetes knowledge questionnaire was distributed. Their HbAlc, frequency of SMBG and diabetes knowledge were reevaluated at 3 months aftercamp. Results. Thirty children (13 males) participated in this camp; $12(40 \%)$ Malays, 11 (36.7\%) Chinese, and 7 (23.3\%) Indians. Mean age at diagnosis was 8.0 (3.8) years while the duration of diabetes was 4.7 (2.7) years. Prior to camp, mean HbAlc was 9.5\% (2.1) and participants performed SMBG 2.0 times per day on average. During camp, hypoglycaemia episodes were common, but there was no severe hypoglycaemia episode. Nobody had diabetic ketoacidosis. At 3 months after the camp, mean HbAlc was $9.9 \%$ (2.1) while mean number of SMBG dropped to 1.5 times per day. There was no significant difference in the percentage of hypoglycaemia, normoglycaemia or hyperglycaemia episodes prior to and after the camp. However, there was a significant improvement $(P=.002)$ in diabetes knowledge after the camp. Conclusion. Most of the participants had poor glycaemic control. Participating in diabetes camp did not show significant improvement in glycaemic control or SMBG but did show marked improvement in diabetes knowledge. Diabetes camp is a valuable programme to impart diabetes knowledge for better management of their diabetes.

\section{The Study on the Preventive Effect and Mechanism of All-Trans Retinoic Acid on Type 1 Diabetes Mice}

\author{
Xing Jie, Wang Ye, and Zhang Li
}

\section{The Second Hospital of Jilin University Pediatrics (Jilin Changchun)}

Objective. To investigate the preventive effect and mechanism of all-trans retinoic acid (ATRA) on T1DM mice model, induced by multiple low-dose streptozotocin (STZ). Methods. Mice were randomly divided into 3 groups. Prevention group were pre treated with ATRA subcutaneous injection, and diabetic group and control group were treated with the mixed solution subcutaneous injection, of 95\% peanut oil and 5\% in same amount dimethyl sulfoxide. Then, prevention group and diabetes group mice were given intraperitoneal injection of multiple low dose STZ to induce T1DM model, while control group was given intraperitoneal injection of citrate buffer solution in the same amount. After a week injection of STZ, we dynamically observed the general state, weight, blood glucose level and incidence of mice. All mice were sacrificed in the end of the 6th week. The pancreas were prepared pathology to observe islet changes, and radioimmunity analysis was used to detect the serum insulin level, while enzyme linked immunosorbent assay was used to determine the levels of IFN-Gamma and IL-4. Results. Mice in prevention group had general state similar to normal mice, decreased blood glucose, increased serum INS, reduced IFN-Gamma levels, elevated levels of IL-4, IFN-Gamma/IL-4 ratio decreased (compared with diabetes group, the difference was significant, $P<.05$ ), to prevent the occurrence of autoimmune insulitis. Conclusion. ATRA has an effect on preventing the incidence of T1DM diabetes induced by multiple low-dose STZ. This effect may depend on ATRA preventing elevated levels of IFNGamma and reducing IL-4 level induced by multiple lowdose STZ. 


\section{Acute Metabolic Cataract as a First Manifestation of Type 1 Diabetes in Children: Report of Two Cases and a Review of the Literature}

\author{
Yan-Yan Jin, Ke Huang, Chao-Chun Zou, \\ Li Liang, and Ji Jin
}

\section{Department of Endocrinology, The Affiliated Children's Hospital, College of Medicine, Zhejiang University, Hangzhou 310003, China}

Objective. To highlight the metabolic cataract as a first manifestation of Type 1 Diabetes in children. Methods. Report of two cases (4 eyes) and review of the literature. Results. Two 11-year-old girls, met the inclusion criteria of Type 1 Diabetes and cataract, were enrolled in our unit in 2008 and 2009. They presented cataracts 1 and 6 months before the diagnosis of diabetes, respectively. Fundus oculi of the second girl's right eyes were invisible due to cataract after mydriasis. No abnormality was found by fluorescein fundus angiography. However, Visual-Evoked Potentials (VEP) and Electroretinogram (ERG) tests changed as follows: peak time of 4 eyes' P100 was slightly longer, amplitude of the a and $b$ wave for the ERG of the first girl's left eye and the second girl's right eye reduced. Both of them havenot been operated on yet, although the second patient's right eye was advised to undergo cataract surgery. Controlling the blood glucose levels by insulin therapy, the first patient had a visual acuity from $20 / 66$ to $20 / 40$ after 3 months, and to $20 / 29$ after 6 months; the second girl's left eye was from 20/50 to $20 / 40$ and the right eye was from 20/180 to 20/125 after 6 months therapy. Literature reviewed showed only 11 cases being reported in 6 papers to have a cataract before the diagnosis of diabetes and most (10/11) of them had a cataract surgery to restore vision, there was only one child completely resolved by controlling blood glucose. Conclusions. Acute metabolic cataract presented as the first manifestation of Type 1 Diabetes is infrequent in children, and those who restored vision through controlling blood glucose have been rarely reported.

\section{A Case of Reducing Insulin Requirement in Type 1 Diabetes after Hepaticojejunostomy}

\section{Ah Reum Kwon, Hyun Wook Chae, Ho-Seong Kim, and Duk Hee Kim}

\section{Severance Hospital, Seoul, Korea}

Some gastrointestinal surgical procedures can induce lose body weight loss, therefore a vast improvement in glycemic control has been observed in type 2 diabetes. However, it is becoming evident that these procedures modulate the gutbrain axis by altering the anatomy of the gut and affecting gut hormones. These alterations of hormonal concentrations may be also able to improve glycemic control. A 12-year and 10-month old girl who has been diagnosed with type 1 diabetes 2 years ago, was admitted for abdominal pain due to choledochal cyst, type IV-A and anomalous union of pancreaticobile duct. Her height, weight, and BMI were $158 \mathrm{~cm}, 54 \mathrm{~kg}, 21.63 \mathrm{~kg}$, respectively, and she underente not obese. She controlled blood glucose by $66 \sim 72$ units of insulin per day, and the HbAlc was $11.0 \%$. She was received hepaticojejunostomy and was discharged after 3 weeks. Interestingly, after discharge, she was able to control blood glucose only 26 32 units of insulin per day, even though her body weight was not changed. The last HbAlc was also $8.8 \%$. Although we were unable to identify the cause of reducing insulin requirement, we thought that this was due to decreased ghrelin and/or increased incretin or unknown hormonal metabolic effect by hepaticojejunostomy. Therefore, she gained reduced insulin requirement and improved glucose tolerance apart from treatment of choledocal cyst. To the best of our knowledge, this is the first case of metabolic surgery in type 1 diabetes without obesity.

\section{Blood Glucose Profiles in Diabetic NOD Mice Using Insulin Glargine and Aspart}

\section{Peng Shao, Guimei Li, Lijuan Zhang, Yanyan Hu, and Caihong Liu}

\section{Provincial Hospital Affiliated to Shandong University, Jinan 250021, China}

Objective. To evaluate insulin glargine and aspart for their ability to normalize the blood glucose profile of diabetic NOD mice. Methods. Thirty newly diagnosed diabetic female NOD mice were randomized into 5 groups $\mathrm{A}, \mathrm{B}, \mathrm{C}, \mathrm{D}$, and $\mathrm{E}$ (each group $n=6$ ). Groups $\mathrm{A}$, $\mathrm{B}, \mathrm{C}$ and $\mathrm{D}$ had free access to food and they were given insulin glargine (I) at 9:00 subcutaneously in different doses:100IU/(god), 50IU/(god),15IU/(god) and PUBS $5 \mu \mathrm{L} / \mathrm{d}$, respectively. Group E was the intensive insulin therapy group: insulin glargine as a basal replacement was administrating subcutaneously at 9:00, and food was given for one hour after insulin aspart (IAN) administrated subcutaneously every four hours (begun at 0300 per day). Also a group (Group $\mathrm{Fa}, n=6$ ) of age matching nondiabetic NOD mice was included in the study. The blood glucose value at different time on the fourth day $(24 \mathrm{~h})$ were recorded, and the effect of each treatment was estimated on the 15th day. Results. The average blood glucose was significantly lower in intensive insulin therapy group E with preprandial IAN plus basal I than in Group BACK and D $(P<.05$, resp. $)$. But there was no significant difference when Group E was compared with Group A and Group $\mathrm{F}(P>.05)$. The fluctuation of blood glucose value in Group E was significantly lower compared with Group A, $\mathrm{B}$ and $\mathrm{C}(P<.05$, resp.). The percentage of normal blood glucose during 24 hours in intensive insulin therapy Group E was much higher than in Group A, B, C, and D. Severe hypoglycemia and hyperglycemia were often observed in those single insulin glargine groups. The death rate on the 15th day of Group A, B, C, and E were 5/6, 3/6, 0/6, and 0/6, respectively because of hypoglycemia. Conclusions. Intensive therapy group E with preprandially IG plus basal IG in NOD 
mice with T1DM is superior with better glycemic control, less hypoglycemic episodes, less blood glucose fluctuates and the higher percentage of normal blood glucose during 24 hours.

\section{Early Intensive Insulin Therapy in Diabetic NOD Mice Using Insulin Glargine and Aspart Decreased Insulitis and Increased Bcl-2 Expression}

\author{
Lijuan Zhang, Guimei Li, Peng Shao, Yanyan Hu, \\ and Caihong Liu
}

\section{Provincial Hospital Affiliated to Shandong University,} Jinan 250021, China

Objective. To investigate the effects of early intensive insulin therapy on insulitis, apoptosis and regeneration of islets Methods. Twelve newly diagnosed diabetic female NOD mice were randomized into 2 groups: early intensive insulin therapy group $(n=6)$ and PBS group $(n=6)$, the treatment of these two groups was initiated within 3 days after onset of diabetes. A PBS control group $(n=6)$ of age matching nondiabetic mice was included in the study. group was sacrificed on the 20th day while the other two groups were killed after 60 days of treatment. Pancreases were removed to examine insulitis scores by $\mathrm{HE}$ straining, and expression of $\mathrm{Bcl}-2$ and Bax on islet cells was detected by immunohistochemical straining. Results. Diabetic symptoms were controlled by early intensive insulin therapy. The mean weight and insulitis score of intensive group were significantly lower than that in the after group $(P=.00, .01$, resp. $)$, and there was no significant difference when compared the other with group $(P>.05)$. The expression of $\mathrm{Bcl}-2$ on islet cells was stronger in intensive insulin therapy group than in the other two groups $(P<.05)$. The Bax expression was stronger in intensive insulin therapy group than in group $(P<.05)$, but there was no significant difference when compared with group $(P=.26)$. Conclusions. Early intensive insulin therapy can decrease insulitis of islets, increase the expression of Bcl-2 and decrease the expression of Bax on islet.

\section{A Randomized Control Trial of Intensive Diabetes Education Intervention in Children with Type 1 Diabetes}

\section{Xiuzhen Li, Li Liu, Liping Fan, Junqing Huang, Wen Zhang, and Xiaojian Mao}

\section{Endocrinology Department of Guangzhou Women and Children's Medical Center, China}

Background. Patient education is a cornerstone of treatment in diabetes mellitus, but there is not unanimity of opinion as to how it should be delivered. Objective. To assess the effects of an intensive diabetes education intervention on diabetes control in children with type 1 diabetes. Methods. 34 children with type 1 diabetes for $>6$ months were randomly assigned to either an intensive $(n=18)$ or a current education $(n=16)$ group. The intensive education group received 2 days of a diabetes education curriculum involving a diabetes specialist, nurse, dietitian and monthly telephone communication with the diabetes team, and attended the diabetes camp for at least one time. The current education group visited the diabetes clinic every 3 months providing basic information on topics related to diabetes management. Changes in $\mathrm{HbAlc}$ and hypoglycemia frequency, were recorded before 3 months and after 6 months starting the intervention. Results. At baseline, there were no differences between groups in age, sex, diabetes duration, $\mathrm{HbAlc}$ and hypoglycemia frequency. In the intensive group, HbAlc improved from $8.6 \pm 0.86 \%$ at baseline to $8.1 \pm$ $0.82(P<.001)$ at 6 month of the intervention. This effect was more distinct in patients with poor glycemic control (HbAlc > 8.5\%). In the current education group, HbAlc did not change from baseline to 6 months $(8.7 \pm 0.9$ versus $8.8 \pm 0.9, P=.82$ ). Hypoglycemia frequency did not change in either group. Conclusions. HbAlc improved significantly after 6 months of intensive diabetes education intervention, especially in those with poor glycemic control $(\mathrm{HbAlc}>8.5 \%)$.

\section{A Retrospective Review of the Diabetic Ketoacidosis in Two Regional Hospitals in Hong Kong}

\section{Po Yee Loung, C N Hung, and Ching Yee Lee}

Department of Paediatrics and Adolescent Medicine, Kowloon West Cluster, Hong Kong

The incidence of Type 1 diabetes mellitus (DM) in our region was increasing over the last decade. Many of them presented with diabetic ketoacidosis (DKA) and require admissions to intensive care unit. DKA is associated with significant morbidity and mortality and probably could be prevented by earlier diagnosis of DM and intervention. This depends on the knowledge of the population on DM and the level of alertness of the primary care doctors. We are interested in the duration of potential window period for early intervention and secondary prevention and the possibility of reducing the rate of DKA in newly diagnosed paediatric diabetes patients. Questionnaires about their first presentation were sent to Type 1 diabetes patients during their attendance in the diabetes clinic in two regional hospitals. Their hospital records were reviewed for comparison to minimize recall bias. Total number of 75 diabetic patients was analyzed with 37 male and 38 female patients. Their mean age at presentation was 8.9 years of age. 44 of them $(60 \%)$ presented as DKA, most of whom required admission to the intensive care unit and insulin infusion. The common presenting symptoms included polyuria $(85.7 \%)$, polydipsia $(85.7 \%)$ and weight loss (71\%). Other symptoms include nocturia, lethargy, constipation and shortness of breath. 
The mean duration of polydipsia and polyuria was 9.8 weeks and 6.3 weeks, respectively. They had seeked medical attention for an average of 2.4 times (maximum of 10) before the diagnosis was made. $49 \%$ of them had their diagnosis made in the Accidental and Emergency Department, $17 \%$ in the Government Clinic while $34 \%$ in the Private Practitioners. The mean duration from the onset of symptoms to the diagnosis, which is the window for early detection and intervention was 6.8 weeks and the median was 4 weeks. This was an invaluable period for the parents and doctors to intervene and reduce the health hazard related to DKA. Diabetic patients presented with DKA were compared to patients without DKA regarding the duration of their symptoms, the blood results and other characteristics in our study. On the other hand, about $15 \%$ patients do not have typical symptoms of polyuria and polydipsia, especially in young children. The diagnosis remains a challenge to the primary care doctors. Simple urine testing in children with unexplained weight loss or respiratory distress is extremely helpful to exclude DM.

\section{Three Patients with Type 1 Diabetes Mellitus Associated with Autoimmune Inflammatory Bowel Disease}

\section{Masahiro Noda, ${ }^{1}$ Kiyomi Abe, ${ }^{1}$ Keisuke Yoshii, ${ }^{1}$ Yasuhiro Naik, ${ }^{1}$ Reiko Horikawa, ${ }^{1}$ Kouichi Kamei, ${ }^{2}$ Shuichi Itou, ${ }^{2}$ Daisuke Ariyasu, ${ }^{3}$ Koji Tsubouchi, ${ }^{4}$ and Kazumoto lijima ${ }^{5}$}

\author{
${ }^{1}$ Division of Endocrinology and Metabolism, \\ National Center for Child Health and Development, Japan \\ ${ }^{2}$ Division of Nephrology, National Center for Child Health \\ and Development, Japan \\ ${ }^{3}$ Division of Endocrinology and Metabolism, \\ Tokyo Metropolitan Children's Medical Center, Japan \\ ${ }^{4}$ Department of Pediatrics, Chuno Kosei Hospital, Japan \\ ${ }^{5}$ Department of Pediatrics, Kobe University Graduate \\ School of Medicine, Japan
}

Autoimmune disorders, such as thyroiditis, celiac disease, and Addison's disease are, known to be associated with type 1 diabetes mellitus (T1DM). Inflammatory bowel disease (IBD) is also a comorbid disorder with T1DM, however, the cooccurrence is rare during childhood. Here we report three patients with T1DM who developed IBD during infancy. Case 1. A 26-year-old man, whose died at 6 months of intractable diarrhoea. He developed T1DM at 6 months old, intractable chronic diarrhoea due to food allergy at 8 months old, and was treated with insulin and steroid. He developed steroid-dependent nephrotic syndrome at 6 years old. We identified FOXP3 gene mutation (IVS1+1G > T) and diagnosed him as having IPEX (immune dysregulation, polyendocrinopathy, enteropathy, X-linked) syndrome. Case 2. A 13-year-old girl, whose developed T1DM at 2 years old. She developed T1DM at the age of ten. She showed frequent vomiting and epigastralgia, and was found to have gastric ulcer since she was 11 years old. She also had hypoproteinemia secondary to proteinlosing gastroenteropathy. She was diagnosed as having autoimmune gastroenteropathy. Case 3. A 11 year-old girl. She developed T1DM at 2 years old, Hashimoto disease at three, and intractable diarrhoea at eleven. She was diagnosed as having Crohn diseases by endoscopy and serological tests. Discussion. The association of IBD is rare in T1DM during childhood. Early onset of T1DM and IBD suggests genetic background in immune system in these patients. T1DM is mostly caused by polygenic reaction against pancreatic island, while monogenic causes are found in autoimmune polyendocrine syndrome type 1 (APS-1) and IPEX. We found FOXP3 mutation in one patient. Early detection and diagnosis of these conditions is essential for the better outcome, since the bone marrow transplantation could be another therapeutic option.

\section{Factors Influencing Chronic Diabetic Complications in Type 1 Diabetes}

\author{
Naree Panamonta, ${ }^{1}$ Tongchai Pratipanwut, ${ }^{1}$ \\ and Ouyporn Panamonta ${ }^{2}$ \\ ${ }^{1}$ Department of Medicine \\ ${ }^{2}$ Department of Pediatrics, Srinagarind Hospital, \\ Faculty of Medicine, Khon Kaen University, \\ Khon Kaen, Thailand
}

Although type 1 diabetes mellitus (T1DM) usually begins in childhood and adolescence, the prevalence of cases with complications is rising in adults as a continuum. The objective of this study is to determine the characteristics and factors influencing chronic complications in patients with T1DM. During 2006-2007, 43 T1DM patients were retrospective reviewed. Collection and analysis of demographic data, and clinical status of diabetes including complications and treatment were performed. There were 16 males (37.2\%) and 27 females (62.8\%). The mean age was17.84 \pm 7.09 years (range $4.08-37.50$ ) and the mean age at onset was11.32 \pm 5.87 years (range $0.92-$ 28.08) while the mean duration of DM was $6.77 \pm 4.28$ years (range 1.08-19.0). The mean HbAlc of the last visit was $9.62 \pm 3.07 \mathrm{~g} / \mathrm{dL}$ (range 5.2-17.6). Self-monitoring of blood glucose (SMBG) was performed in 21 patients (48.84\%). Acute complications (DKA and hypoglycemia) were documented in 29 patients $(67.44 \%)$, while chronic (microvascular and macrovascular) complications were documented in 13 patients (30.23\%). Older age of onset and longer disease duration were the factors associated with chronic diabetic complications ( $P=.004$ and 0.006 , resp.). The study showed no statistical significance in the HbAlc level, the frequency of daily insulin injections, and the presence of SMBG between the patients with and without complications. Females tended to have the greater risk to develop complications even though there was no statistical difference. Our results suggested that prompt evaluation and proper management of DM could prevent these complications. 


\section{Psychological Characteristics of Children and Adolescents with Type 1 Diabetes Mellitus}

\begin{abstract}
Young Jun Rhie, Jeong Hee Shin, Yeon Joung Oh, So Hee Eun, Ji Yeon Kim, and Kee Hyoung Lee
\end{abstract}

\author{
Department of Pediatrics, Korea University \\ College of Medicine, Korea
}

Objectives. Chronically ill persons have an increased risk of greater psychological morbidity compared with general population. Type 1 diabetes mellitus (T1DM) is a chronic disease. Children and adolescents with T1DM are at particular risk for psychological problems which are associated with poor metabolic control and complications. The purpose of this study was to examine the presence of behavioural and emotional problems in children and adolescents with T1DM. Methods. Subjects with T1DM aged 6 to 18 years $(n=37)$ and age-matched normal healthy controls $(n=36)$ were evaluated using Korean Child Behaviour Checklist (K$\mathrm{CBCL}$ ) and Children's Depression Inventory (CDI). Results. Children and adolescents with T1DM had higher scores in CDI, compared with normal controls $(13.0 \pm 8.5$ versus $6.3 \pm$ 5.3). T1DM patients had more tendency to show depression, social problem, attention problem, delinquent behaviour, aggressive behaviour, internalizing problem, externalizing problem, and total behaviour problems of K-CBCL than controls. There were no significant differences in CDI and K-CBCL between well-controlled (HbAlc $<9 \%, n=16$ ) and poorly controlled (HbAlc $\geq 9 \%, n=21$ ) patients, and intensive $(n=13)$ and conventional $(n=24)$ therapy patients. Conclusions. Children and adolescents with T1DM are prone to behavioural and emotional problems. Psychological evaluation and intervention are required in the management of T1DM during childhood and adolescence.

\section{Experience of Basal-Bolus Administration in Type 1 Diabetes Mellitus Adolescent Previously Treated with Conventional Therapy: Limited Resources Perspective}

Frida Soesanti, Jose RL Batubara, Bambang Tridjaja, and Aman Pulungan

Department of Child Health, Medical School-University of Indonesia, CiptoMangunkusumo Hospital, Indonesia

The management of type $1 \mathrm{DM}$ in Indonesia is still a big challenge caused by unavailability of insulin, limited access to medical helps, low income, no health insurance, and low education level. The DCCT showed that intensive therapy (basal-bolus regimen and continuous insulin infusion) slowed the progression of microvascular complications, significantly. Institution of basal-bolus therapy requires higher cost and understanding of insulin and carbohydrate effect on blood glucose. Thus it is a challenge to most of our patients from low socioeconomic and lower education level. Here we present an observational study on five adolescents aged 15-17 years who have been diagnosed type 1 DM for more than two years and previously treated with premixed insulin. We compared HbAlc level, presence of hypertension, microalbuminuria, retinopathy, diabetic ketoacidosis, insulin compliance, hypoglycemia episode, and frequency of blood glucose monitoring 6 months prior and 6 months after institution of basal-bolus regimen. Six months prior, 2 patients had HbAlc levels above 16\%, 2 patients $13 \%$, and one patient $8.5 \%$. Three patients had microalbuminuria, two suffered from hypertension and 1 patient had diabetic retinopathy. All of the patients had more than 2 episodes of DKA and irregular blood glucose monitoring. The patients were given rapid insulin as bolus and detemir once daily as basal insulin. Six months after basal-bolus regimen, the HbAlc level declined in all patients. Two patients who had HbA1c levels above $16 \%$ gained the biggest improvement to $7.9 \%$ and $13 \%$, respectively. The decreasing point of HbAlc level varied from $0.4 \%$ to $9.1 \%$. The hypertension was controlled, while the microalbuminuria and retinopathy resolved. The hypoglycemia episode also decreased. Only one patient suffered from DKA because of unavailability of detemir. This study showed us that despite of the limitations of all resources including funding, knowledge, and health facility, the institutions of basal bolus regimen still resulted in better glycemic control and slowed the progression of micro vascular complications. This study has pushed us to move forward on the management of type $1 \mathrm{DM}$ to cross boundaries to achieve optimal growth and development.

\section{Type 1 Diabetes with Premature Ovarian Failure}

\author{
Hemlatha Subramaniam and Rahmah Rasat \\ Hospital University Kebangsaan Malaysia
}

Introduction. Failure of two endocrine systems is uncommon and presents diagnostic and management challenge. We report a case of a young woman with Type 1 Diabetes and premature ovarian failure. Methods. A 15-year-old girl diagnosed as Type 1 diabetes at the age 2 years presented with poor control of diabetes and frequent hypoglycemia for trivial activity. Developmental milestones and behaviour are appropriate for age. No history of short stature in the family. Patient's mother and older sister attained menarche at 12 years old. Results. Physical examination showed no facial dysmorphism, with height of $141.3 \mathrm{~cm}(<\mathrm{P} 3)$ and weight of $43.7 \mathrm{~kg}(<\mathrm{P} 25)$. Patient had no secondary sexual characteristic. Karyotyping showed 46XX with no SRY gene. Investigation revealed poor insulin reserve and elevated HbAlc level of more than $10 \%$. Patient is clinically and biochemically euthyroid with no thyroid auto antibodies. Short Synacthen test showed normal cortisol response of $>500 \mathrm{mmol} / \mathrm{L}$. Basal $\mathrm{LH}$ and $\mathrm{FSH}$ are elevated with low estradiol which is consistent with hypergonadotrophic hypogonadism. Ultrasound of pelvis showed atrophied ovaries. Conclusion. Elevated gonadotrophins on more than one occasion, in an amenorrhoeic patient, usually indicated permanent ovarian failure. About $10-30 \%$ of women with POF have a concurrent autoimmune disease, most common 
is hypothyroidism. Premature ovarian failure in patient with Type 1 Diabetes is a rare occurrence.

\section{Clinical Appearance of Diabetic Ketoacidosis Patients in Pediatric Department of Saiful Anwar Hospital, Malang, Indonesia, 2005-2009}

\section{Harjoedi Adji Tjahjono}

\section{Saiful Anwar Hospital}

Objective. To present the clinical appearance of diabetic ketoacidosis in patients who were diagnosed in the Pediatric Department of Saiful Anwar Hospital between 2005 and 2009. Methods. A descriptive study of clinical appearance of diabetic ketoacidosis of 19 patients, 1-14 years of age, diagnosed in Pediatric Department of Saiful Anwar Hospital between 2005 and 2009. Results. From the 19 patients, 58\% were girls. $53 \%$ were between 5 and 10 years of age. $37 \%$ had an equal frequency between $400-600 \mathrm{mg} / \mathrm{dl}$ and 600 $800 \mathrm{mg} / \mathrm{dl}$ of blood glucoses levels. 63\% were undernourished. About 53\% had moderate DKA. 53\% patients had blood bicarbonate concentrations of 5-9, $9 \mathrm{mmol} / \mathrm{L}$. Blood osmolarity levels were $>295-400 \mathrm{mOsm} / \mathrm{l}$ in $84 \%$ patients. $53 \%$ had blood potassium levels of 3, $5-5 \mathrm{mmol} / \mathrm{L}$. $42 \%$ patients had leucocyte counts of $10.000-<20.000 /$ ul. The most common symptoms of those patients were vomiting, abdominal pain, decreased of consciousness, seizures, diarrhoea, decreased of body weight, polyuria, polydipsia, polyphagi, and febris. The majority complained of polyuria about $16 \%$. The majority patients of DKA who had blood ketone levels of more than $5 \mathrm{mmol} / \mathrm{l}$ were $67 \% .61 \%$ had ketonuri of $4+$. The majority of those patients had anion gap levels of about 12.0-24.0 (63\%). Conclusions. Of these 19 patients the majority were girls, aged between 5 and 10 years. The majority of patients had high blood glucose levels (between $400-600 \mathrm{mg} / \mathrm{dl}$ and $600-800 \mathrm{mg} / \mathrm{dl}$ ). Most patients were undernourished and had moderate DKA; the majority had blood bicarbonate concentrations of about $5-9.9 \mathrm{mmol} / \mathrm{l}$ and high blood osmolarity levels (>295-400 mOsm/l). And most complained of polyuria and had high blood ketone levels ( $>5$ ), potassium levels $3.5-5 \mathrm{mmol} / \mathrm{l}$, and leucocyte counts of $10.000-20.000 \mathrm{u} / 1$.

\section{Efficacy and Safety Comparison between Traditional 24-Hour Fluid Therapy and 48-Hour Fluid Equilibrium Therapy on Diabetic Ketoacidosis in Children and Adolescents}

\section{Di Wu, Chunxiu Gong, Bingyan Cao, and Yi Gu}

\section{Beijing Children's Hospital, Capital Medical University}

Objective. Compare the safety and efficacy of a 48-hour fluid equilibrium therapy with 24 hours in Diabetic Ketoacidosis children and adolescents with moderate and severe degree of dehydration. Methods. Choose 36 DKA patients who were moderate and severe degree of dehydration, divided them into 48-hour fluid equilibrium therapy group $(n=27)$ and 24 hours group $(n=9)$, to compare the time difference of disappearance urine ketones, change of corrected sodium and effective osmolality with plasma glucose decreasing, and prognosis of the patient with cerebral oedema and hernia. We use statistics software SPSS16.0 to separate $t$-test and $X^{2}$ analysis. Result. (1) There is no statistical significance variance on the time of disappearance urine ketones in both subgroup. 48-hour group: $(X \pm \mathrm{SD}) 21.7 \pm 9.01 \mathrm{~h}$, $24 \mathrm{~h}$ group: $19.6 \pm 10.61 \mathrm{~h},(P=.582)$. (2) With the plasma glucose decreasing, corrected sodium reduced $1.5 \pm$ $5.70 \mathrm{mmo} / \mathrm{L}$ in 48 -hour group while $12.8 \pm 4.93 \mathrm{mmo} / \mathrm{L}$ in 24-hour group, $(P=.015)$, and the effective osmolality reduces, respectively $7.2 \pm 11.97 \mathrm{mosm} / \mathrm{L}$ in 48 -hour group and $26.5 \pm 5.61 \mathrm{mosm} / \mathrm{L}$ in 24 -hour group, $(P=.039)$. (3) For 2 cases whose CT shows cerebral oedema and hernia, the cerebral oedema and hernia of the one in 48 hours fluid administration group disappear after 38 hours, the other in 24-hour fluid administration group is dead after 11 hours of admission to hospital. Conclusion. For the patient of DKA with moderately and severe dehydration, excessive rates of fluid administration exacerbate the risk of cerebral oedema. For more severe DKA with more severe degree of dehydration, 48 hours fluid equilibrium therapy should be adopted in order to avoid the risk of cerebral oedema and herniation. During the intravenous fluid, rapid decline of corrected sodium and effective osmolality may induce cerebral oedema. So the change of corrected sodium and effective osmolality should be monitored.

\section{Central Brain Herniation during Treatment of Diabetic Ketoacidosis in Children with Newly Onset Diabetes}

\section{Xin Ying, Yang Min, and Tong Ya Jie \\ Department of Pediatrics, Shengjing Hospital of China Medical University, Shenyang, China}

Objectives. Severe cerebral oedema resulting in central brain herniation is uncommon in DKA management, but its mortality is very high. Early recognition and treatment of cerebral oedema in DKA is vital for patient management. Methods. Cases description and review of literature. Results. A previously healthy, 7-year-old girl came to our hospital for polydipsia, polyuria, and body weight loss for 10 days. Laboratory analysis showed that her blood glucose level was $15.3 \mathrm{mmol} / \mathrm{L}$, pH 7.16, BE-18.7 mmol/L, ketone $2+$, and the patient was diagnosed with DKA. She was treated with intravenous fluid resuscitation and intravenous insulin. Four hours later, her blood glucose decreased to $6.3 \mathrm{mmol} / \mathrm{L}$, but the acidosis condition was not improved, and she felt headache occasionally. Seven hours after therapy, the acidosis condition still was not improved, $\mathrm{pH}$ 7.14, BE $-20.3 \mathrm{mmol} / \mathrm{L}$, ketone $3+$, and her headache became a little bit more severe, accomplished by vomiting. The intravenous fluids were restricted. Nine hours after therapy, she fell into convulsions suddenly, and mannitol was administered immediately. Fifteen minutes later, she became coma, and 
her pupils were not symmetric, central brain herniation was suspicious, and she was transferred to PICU. She received a total of $950 \mathrm{ml}$ of normal saline and 5\% dextrose with normal saline in her first 9 hours after admission. Her serum natrium decreased from $139 \mathrm{mmol} / \mathrm{L}$ to $128 \mathrm{mmol} / \mathrm{L}$, and $3 \%$ natrium chloride was administered. She convulsed again, had fixed and dilated pupils and a Glasgow coma scale of 6. She had irregular respiration and $\mathrm{SaO} 2$ decreased; mechanical ventilation was performed. An emergent CT scan revealed marked low-density changes in the temporal lobe, occipital lobe, and subfrontal area. The patient was died at $54 \mathrm{~h}$ after admission. The second patient a 10 -year-old girl presented to an outside hospital complaining of respiratory distress, abdominal pain, and malaise. Her blood glucose level was $52.5 \mathrm{mmol} / \mathrm{L}$, CO2CP $8 \mathrm{mmol} / \mathrm{L}$, ketone $3+$, and she was diagnosed with DKA. The patient was treated with intravenous insulin and fluid resuscitation. Several hours later, her blood glucose decreased and acidosis was corrected, but she went into a coma. She was transferred to our hospital. On admission, the patient had fixed and dilated pupils and Glasgow coma scale of 5. CT scans of the head showed diffuse low-density changes in the right temporal lobe, basal ganglia, and occipital lobe. During 2-week therapy, her condition was not improved and kept on coma; her muscle tone was increased, and she was discharged. Conclusion. Patients who develop cerebral oedema in DKA management may deteriorate rapidly; early recognition and initiating prompt intervention are very important.

\section{Systemic Expression of IL-10 Gene Prevents the Onset of Type 1 Diabetes in NOD Mice}

\section{Ai-jing $\mathrm{Xu}$, Zhi-hong Chen, Fei Tian, Li-hua Yan, and Tang Li \\ Department of Pediatrics, the Affiliated Hospital of Qingdao Medical College, Qingdao University, Qingdao 266003, China}

Background. Studies of animals with type 1 diabetes have revealed that auto reactive $\mathrm{T}$ cells that mediate islet beta cell destruction can be manipulated by the administration of $\mathrm{Th}(2)$ cytokines. Using gene delivery to express the targeted protein can overcome the need for frequent administration of cytokines on account of their short half-lives. In this study, the effect of IL-10 gene delivery was evaluated in vivo using an adenovirus vector (Ad) constructed with a murine IL-10 cDNA, and apoptosis was detected by TUNEL assay. Methods. To evaluate in vivo efficacy, Ad-mIL-10 was intraperitoneally injected into 4-week-old and 9-week-old NOD mice and compared to the transduction using the vector only (Ad-EGFP) and untreated control. Immunohistochemistry and Western blotting analysis were used to detect the mIL-10 expression. Blood glucose concentration was measured twice every week. After mice were killed on 32w, serum insulin, IL-10, IFN-gamma, TNF-alpha, and IL-6 level were measured by enzyme-linked immunosorbent assay (ELISA). Pancreatic histology was measured for determination of insulitis grades. Apoptosis was measured by using a TUNEL assay. Expressions of Fas, Bcl-XL, and caspase-3 were estimated by Immunohistochemistry analysis. Results. Treatment with Ad-mIL-10 resulted in the retention and expression of the vector for longer than 5 weeks, associated with a considerable elevation in the plasma level of IL-10, which was not observed in Ad-EGFP-treated mice and untreated control group. In IL-10-treated NOD mice, the glucose level was greatly lower than Ad-EGFP-treated group and untreated control group $(P<.05)$ and the insulin level was higher than the other two group $(P<.05)$. The incidence of diabetes was markedly reduced in NOD mice injected with Ad-mIL-10 as compared with those injected with Ad-EGFP or as compared with nontreated NOD mice $(P<.05)$. The serum levels of IFN-gamma, TNF-alpha, and IL-6 were decreased in IL-10-treated group. The insulitis grades of IL-10-treated mice were lower than that of Ad-EGFP-treated group $(P<.01)$. Treatment with Ad-mIL-10 significantly decreased apoptosis compared to control $(P<.05)$ by TUNEL assay. Decreased expression of Fas and caspase- 3 and increased expression of bcl-xL were observed in the presence of Ad-mIL-10. Conclusions. This study indicates the potential for IL-10 gene therapy to prevent type 1 diabetes and implicates that IL-10 has a dual function, to inhibit proinflammatory cytokine production and to suppress spontaneous apoptosis in vivo.

\section{Clinical Characteristics of Childhood Diabetes in a Single Center}

\section{Jeesuk Yu, Jong Seok Oh, So Young Kang, In Chang Seong, and Ji Yoon Park}

\section{Department of Pediatrics, College of Medicine, Dankook University, Cheonan, Korea}

Most childhood diabetes is usually thought as type 1 diabetes (T1DM), but there is an increasing tendency of type 2 diabetes (T2DM) even in childhood. Sometimes, it might not be easy to determine the type of diabetes and to choose the best treatment modality. In this study, we retrospectively reviewed the medical records of the patients who was diagnosed as diabetes and followed at the department of Pediatrics, Dankook University Hospital to find the clinical and laboratory characteristics. Total 42 patients were enrolled in this study. There was a little female predominance $(\mathrm{M}: \mathrm{F} 1: 1.33)$. T1DM had an earlier onset age, as expected. Diabetic ketoacidosis (DKA) was the presenting symptom in $58 \%$ of T1DM, but no DKA in T2DM as an initial symptom. Initial and follow-up HbA1c did not show statistically significant differences between the group. Initial serum c-peptide or insulin levels were lower in T1DM. Autoantibody positivity was high in T1DM, implying it as the very valuable marker in T1DM. Obesity was not common in childhood T2DM. Oral hypoglycaemic agents with or without insulin were chosen as a treatment in T2DM. In one patient who had been initially diagnosed as having T1DM since 14 months of age, initial insulin injection was changed to combination therapy (long-acting insulin injection plus oral insulin secretagogue) because 
there was no autoantibody found and the patient showed fluctuating hypoglycemia. After then, there was no more hypoglycaemic event and HbA1c levels could be maintained below $6.5 \%$, suggesting the importance of specific diagnosis. Adolescent period was thought as a very vulnerable period to manage the diabetes requiring more intensive emotional support including family cooperation. In this study, we can confirm that the autoantubody test is very valuable to diagnose specific type of diabetes, and specific diagnosis is very important for better management of the diabetes in childhood and adolescence.

\section{Clinical Analysis of 198 Cases of Children with Diabetes Mellitus}

\section{Wen Zhang, Li Liu, Xiuzhen Li, Yonglan Huang, and Jing Cheng}

\section{Guangzhou Women and Children's Medical Center}

Objective. To investigate the clinical characteristics and provide theoretic basis for the diagnosis and treatment. Methods. To retrospectively analyze 198 new cases of children with diabetes mellitus from January 1999 to March 2009 in our hospital. Results. Among total 198 new cases of children with diabetes mellitus, there were 97 boys and 101 girls and no gender difference existed; the onset peak age was 5 to 6 years old and 9 to 11 years old; new cases were increasing year by year and cases of 2008 were 3.7 times more than those of 1999; among the 198 new cases, 174 cases with type 1 diabetes mellitus were occupying $88.9 \%$, 7 cases with type 2 diabetes mellitus occupying 3.5\%, 14 cases with neonatal diabetes mellitus occupying $7.1 \%$, and 3 case with other types occupying $1.5 \%$. Among the new cases with type 1 diabetes mellitus, the incidence of diabetic ketoacidosis was $42.1 \%$; 55 cases had infection history before the onset of disease; the incidence of diabetic ketoacidosis had significant difference between the infection group and the noninfection group $(P<.01) ; 23$ cases there were family history. 2 cases with hyperthyroidism, 31 cases with temporary hypothyroidism, 30 cases with liver function damage, 12 cases with kidney function damage, 48 cases with dislipidemia, and 27 cases with proteinuria; the average HbA1c was $12.0 \% \pm 1.8 \%$; in 25 cases of type 1 diabetes mellitus, the positive rate of islet cells antibodies (ICA) was $28 \%$, the positive rate of insulin auto-antibody (IAA) was $20 \%$, and the positive rate of glutamic acid decarboxylase antibody (GADA) was 72\%. Conclusion. New cases with diabetes mellitus were increasing year by year and most cases were type 1 diabetes mellitus; cases with neonatal diabetes increased significantly; children with diabetes mellitus had also temporary hypothyroidism, liver function damage, kidney function damage, and dislipidemia; the positive rate of GADA was the highest in autoimmune antibodies; among the first-diagnosis cases with type 1 diabetes mellitus, the incidence of diabetic ketoacidosis had significant difference between the infection group and the noninfection group; diabetic ketoacidosis was the important reason of children with type 1 diabetes mellitus going to hospital.

\section{Type 2 Diabetes and Obesity}

\section{Metabolic Syndrome in 7-12-Year-Old Overweight/Obese Children: A Hospital-Based Study}

\author{
Azriyanti Anuar Zaini and Fatimah Harun \\ Department of Pediatrics, Faculty of Medicine, \\ University Malaya, Kuala Lumpur, Malaysia
}

Introduction. The most significant complication of childhood obesity is the development of metabolic syndrome leading to cardiovascular disease and diabetes mellitus. The aim of this study is to determine the prevalence of metabolic syndrome and diabetes mellitus in overweight/obese children. Methods. A total of 60 overweight/obese and 25 normal weight children were studied. Obesity was defined as those children whose BMI was $>95$ th centile and, overweight $>85$ th centile for their age and sex. All children were subjected to a physical examination and their weights, heights, BMI, waist circumference, and presence of acanthosis nigricans were documented. Fasting blood glucose, fasting insulin, and lipid profile were taken. Oral glucose tolerance tests (OGTT) were done in overweight/obese children only. Metabolic syndrome was diagnosed using the International Diabetes Federation (IDF) definition. Results. Overweight/obese children were significantly taller $(141.3 \pm 12.4 \mathrm{~cm} ; P=.001)$, had higher systolic blood pressure (114.5 $\pm 12.8 \mathrm{mmhg} ; P=.049)$, lower HDL $(1.20 \pm 0.24 \mathrm{mmol} / \mathrm{L} ; P<.001)$ and fasting insulin $(16.5 \pm 12.3 \mu / \mathrm{L} ; P<.001)$ levels, had bigger waist circumference $(87.3 \pm 11.5 \mathrm{~cm} ; P<.001)$, had acanthosis nigricans and an abnormal HOMA index. The prevalence of metabolic syndrome in the overweight/obese children was $25 \%$. Impaired glucose tolerance was present in $6.9 \%$ of obese/overweight children, while $1.7 \%$ had asymptomatic type 2 diabetes Mellitus. Conclusion. This study demonstrated that a significant number of the 7-12-year-old overweight/obese children had metabolic syndrome and risk of diabetes mellitus at a very early age in life. Childhood obesity should be regarded as a serious medical disorder. Vigorous intervention programme either with primary measures or pharmacotherapy should be considered to prevent diabetes.

\section{The Relationship between the Dyslipidemia and Early Vascular Lesions in Obese Children and Adolescents}

\author{
Chao Chun Zou, ${ }^{1}$ Li Liang, ${ }^{1}$ Jun Fen Fu, ${ }^{1}$ \\ Lan Qiu LV, ${ }^{2}$ Pei Ning Liu, ${ }^{3}$ Ke Huang, ${ }^{1}$ \\ and Chun Lin Wang ${ }^{1}$

\section{${ }^{1}$ The Children's Hospital of Zhejiang University School of Medicine \\ ${ }^{2}$ Ninbo Women and Children's Hospital} \\ ${ }^{3}$ Yuying Children's Hospital of Wenzhou Medical College
}

Objective. Obese children and adolescents often have abnormalities of lipid and glucose metabolism, which are 
associated with adulthood hypertension, diabetes, and cardiovascular disease. In this study, the blood lipids, blood pressure, and intima media thickness (IMT) in obese children and adolescents were measured to investigate the relationship between the dyslipidemia and early vascular lesions. Methods. A total of 580 obese children and adolescents aged from 7 to 17 years of age were enrolled from 3 hospitals from January 2008 to September 2009. They were divided into 2 groups according to their blood lipoid levels. Ortholiposis group included 100 males and 52 females with a mean age of 10.47 years and a mean body mass index (BMI) of $28.28 \mathrm{~kg} / \mathrm{m}^{2}$. Dyslipidemia group included 305 males and 123 females with a mean age of 10.83 years and a mean BMI of $27.60 \mathrm{~kg} / \mathrm{m}^{2}$. Physical examination, and measurement of blood lipid, glucose, and liver enzyme were taken. Carotid IMT was measured for 285 subjects. Results. (1) Hypertension was found in $12.5 \%$ (19/152) and $20.1 \%$ (86/428) patients in ortholiposis and dyslipidemia groups, respectively, with a statistical difference $\left(\chi^{2}=4.362, P=\right.$ $.037)$. The OR was 1.760 with $95 \%$ confidence interval of 1.030-3.008. Higher prevalence of hypertension was found in patients with dyslipidemia. (2) The left, right, and mean common carotid IMTs of dyslipidemia group were higher than these of ortholiposis group without statistical difference (all $P>$.05). The left, right and mean internal carotid IMTs in dyslipidemia group were $0.66 \pm 0.15 \mathrm{~mm}, 0.65 \pm$ $0.15 \mathrm{~mm}$ and $0.65 \pm 0.15 \mathrm{~mm}$, respectively, while these in ortholiposis group were $0.62 \pm 0.13 \mathrm{~mm}, 0.60 \pm 0.13 \mathrm{~mm}$, and $0.61 \pm 0.12$, respectively with significant differences (all $P<$.05). (3) Bivariate correlation analysis showed that systolic blood pressure was positively correlated with age, BMI, BMI Z score, waist circumference, hip circumference, uric acid, alanine transarninase, triglyceride, fasting insulin, and insulin resistance index (all $P<.05)$. Moreover, mean carotid and internal carotid IMTs were positively correlated with age, BMI, waist circumference, hip circumference, and triglyceride (all $P<.05$ ). Multiple linear regression analysis showed that hip circumference and insulin resistance index were independent determinants of systolic pressure. Waist circumference was an independent determinant of mean common and internal carotid IMT, and triglyceride was an independent determinant of mean internal carotid IMT. Conclusion. (1) Vascular lesions, including hypertension and thicker tunica intima are common in obese children and adolescents. (2) Vascular lesions are closely related with dyslipidemia, and waist circumference and hypertriglyceridemia are the risk factors.

\section{Abnormal Glucose Homeostasis and Insulin Resistance Are Highly Prevalent in Obese Thai Children and Adolescents}

\author{
Pontipa Engkakul, Sunisa Saowan, \\ Preamrudee Poomthavorn, \\ Pat Mahachoklertwattana, and Patcharin Khlairit
}

Department of Pediatrics, Faculty of Medicine, Ramathibodi Hospital, Mahidol University, Bangkok, Thailand
Background. The prevalence of pediatric obesity has been increasing worldwide. Obesity contributes to insulin resistance, impaired glucose tolerance (IGT), dyslipidemia, and hypertension. Metabolic syndrome (MetS) is associated with childhood obesity. In comparison with studies in Caucasians, previous studies in Asian adults showed a greater proportion of abnormal glucose tolerance at less degree of obesity. Asian children with obesity may carry such a high risk in developing abnormal glucose tolerance and MetS. Objective. To determine glucose homeostasis status and insulin sensitivity in obese Thai children. Methods. Obese children, aged 518 years, were enrolled. Weight, height, body mass index (BMI), and waist circumference (WC) were obtained. All of them underwent an oral glucose tolerance test (OGTT) with glucose and insulin levels measurement. Insulin sensitivity indices were calculated from the measured glucose and insulin levels. MetS was diagnosed using International Diabetes Federation (IDF) criteria. Data are presented in mean (SD). Results. There were 150 obese children ( 76 males), aged 11.2 (2.6) years included. There were 105 children (70\%) who had already entered puberty. Weight and height Zscores were $5.0(2.6)$ and $1.2(1.4)$, respectively. BMI was $28.6(4.8) \mathrm{kg} / \mathrm{m}^{2}$ and BMI Z-score was 3.0 (1.4). WC was 90.5 $(11.8) \mathrm{cm}$ and WC percentile was at 108.1 (22.2). Median (range) homeostasis model assessment of insulin resistance (HOMA-IR) was $3.0(0.4-16.3)$ and whole body insulin sensitivity index (WBISI) was $2.5(0.5-12.0)$. One hundred and nineteen children (79\%) had abnormal OGTT results (81 hyperinsulinemia, 33 impaired fasting plasma glucose and/or IGT and 5 diabetes). Thirty-eight children with IGT and diabetes had BMI and BMI Z-scores of 28.9 (4.2) and 2.9 (1.0), respectively. There were no differences in age, gender, pubertal status, weight, and BMI Z-scores and WC percentile between obese children with normal and abnormal OGTT results. However, children with abnormal OGTT result had higher HOMA-IR and lower WBISI than those with normal OGTT result $(P<.001)$. MetS was documented in 20 children. No differences in age, gender, and pubertal status between children with MetS and non-MetS were shown. However, MetS children had greater weight and BMI Zscores, fasting insulin and insulin resistance as compared to those without MetS. Conclusion. Our study demonstrates a frequent abnormal glucose homeostasis in obese Thai children and adolescents as high as $80 \%$ of those screened for. In comparison with Western studies, the lower BMI threshold in developing an abnormal glucose homeostasis in obese Thai children is documented.

\section{The Prevalence of Hyperinsulinemia and Its Relationship to Fasting Blood Glucose and Two-Hour Plasma Glucose among Overweight and Obese Filipino Children and Adolescents}

\section{Cynthia Garcia-Feliciano and Susana Padilla-Campos}

\section{Philippine General Hospital}

Hyperinsulinism has been regarded to play a crucial role in the pathogenesis of Insulin Resistance Syndrome which is 
strongly associated with obesity. Understanding the impact of hyperinsulinism and its pathogenesis is essential to optimally address the problem. The objective of the study is to determine the prevalence of hyperinsulinemia among overweight and obese Filipino children and adolescents and its relationship to fasting blood glucose (FBG) and two-hour plasma glucose. This is a cross-sectional study with consecutive sampling wherein overweight and obese Filipino children and adolescents aged 10-19 years old with BMI $>85$ th percentile for age and sex, weight for height $>85$ th percentile using the standard BMI chart set by Center for Disease Control (CDC) were tested for fasting and two-hour plasma glucose with simultaneous insulin levels. Fasting lipid profile was obtained in all subjects. The prevalence of hyperinsulinemia was calculated and its relationship to fasting and two-hour plasma glucose was reviewed. The prevalence of hyperinsulinemia among the overweight and obese subjects is $82 \%$ (95\% CI: $67.42-$ 1.49). High insulin levels implying insulin resistance were seen in $81 \%$ of subjects with normal fasting plasma glucose and in $61 \%$ of subjects with normal two-hour plasma glucose. There was no correlation found between blood glucose and insulin levels. The prevalence of T2DM is 2.2\% (95\% CI: $0.0562-11.7704)$ while the prevalence of impaired plasma glucose is $4.4 \%$ at $95 \%$ CI $(0.5428-$ 15.1493). High density lipoproteins (HDL) were low in $100 \%$ of subjects. There was a significant correlation found between BMI and two-hour plasma glucose at 0.05 level of significance. In conclusion, the prevalence of hyperinsulinemia among overweight and obese subjects is $82 \%$ at $95 \%$ CI (67.42-91.49). Since $82 \%$ of our overweight and obese subjects have normal fasting blood glucose associated with high insulin levels, and hyperinsulinemia has been implicated in the metabolic syndrome, it is recommended that simultaneous insulin levels be included with the FBG when screening these individuals for diabetes.

\section{Association of CRP and White Blood Cell Count with Components of Metabolic Syndrome in Children}

\author{
Fangfang Gao, ${ }^{1}$ Geli Liu, ${ }^{2}$ Qingyan Yang, ${ }^{2}$ \\ Rongxiu Zheng, ${ }^{2}$ and Pengli Bao ${ }^{2}$ \\ ${ }^{1}$ Tianjin Medical University \\ 2 Tianjin Medical University General Hospital of Pediatrics
}

Objective. To investigate Association of C-reactive protein (CRP) and white blood cell count (WBCC) with components of metabolic syndrome (MetS) in children. Methods. A total of 70 subjects, 49 boys and 21girls aged 8-15 years, were enrolled. The subjects were divided into four quartiles according to WBCC (WBCC1-4, from the lowest to highest WBCC) in both genders. The mean values of MetS components for each group were compared in boys and girls separately. Age and BMI were adjusted using a multiple linear regression compared to WBCC and MetS-related components of relevance. Bivariate correlation analysis was used to compare the relationship between (CRP) and MetS. Results. The boys had significantly higher WBCC, CRP, BMI, WC, and triglyceride (TG) than the girls. While there were no significant differences in the systolic blood pressure (SBP), diastolic blood pressure (DBP), fasting blood glucose (FPG), high-density lipoprotein cholesterol (HDL-C), lowdensity lipoprotein cholesterol (HDL-C), total cholesterol (TC). When comparing the risks of the quartiles of WBCC, WBCC4 was significantly higher in the BMI and WC than WBCC1, in addition TG of WBCC4 was significantly higher than WBCC1 and WBCC2 while HDL-C of WBCC4 was lower than WBCC1 and WBCC2 in boys. WBCC were positively related with BMI, WC, TG and negatively related with $\mathrm{HDL}-\mathrm{C}$ among boys, whereas in girls only BMI and WC was positively related with WBCC. We also find that SBP, FPG, BMI, and WC were positively associated with CRP among girls; whereas in boys only BMI, WC, and TG was positively correlated with CRP. Conclusions. Children with metabolic syndrome are in chronic inflammatory state. WBCC and CRP as inflammatory markers are predictors of metabolic syndrome and cardiovascular disease.

\section{Changes of HVA Calcium Channel Characteristics and their Relationship with Pancreatic Islet B Cell Parasecretion in Neonatal Rat Born Small for Gestational Age}

\author{
Mengzao He, ${ }^{1,}{ }^{3}$ Yanyan Jin, ${ }^{1}$ Li Liang, ${ }^{1}$ \\ Zhenyong $\mathrm{Wu}^{2}{ }^{2} \mathrm{Xiumin} \mathrm{Wang}^{1}$ and Yanping $\mathrm{Xu}{ }^{1}$ \\ ${ }^{1}$ Department of Endocrinology, The Children's Hospital of \\ Zhejiang University School of Medicine \\ ${ }^{2}$ Department of Neurobiology/Institute of Neuroscience, \\ Key Laboratory of Medical Neurobiology of Ministry of \\ Health of China, Zhejiang University School of Medicine \\ ${ }^{3}$ Department Of Pediatrics, The Second Hospital Of \\ Jiaxing
}

Objective. To determine whether intrauterine malnourishment affects pancreatic islet B cell secretion and highvoltage-activated (HVA) calcium channel characteristics in neonatal rat born small for gestational age (SGA), then to study the association between changes of HVA calcium channel characteristics and B cell secretion variation in SGA neonatal rats. Methods. SGA neonatal rat model was created by reducing maternal total food intake to $50 \%$ of ad libitum during pregnancy in mother rat $(10 \mathrm{~g} / \mathrm{d})$. Neonatal rats obtained from pregnant rats fed ad-lib were defined as group AGA. The pups (10 per group) were killed and their pancreases were extracted within 24 hours after birth. Then pancreatic islets were isolated by collagenase $\mathrm{P}$ digestion $(1 \mathrm{mg} / \mathrm{ml})$ and single islet cells were isolated by dispase digestion $(0.3 \mathrm{mg} / \mathrm{ml})$. Whole cell patch clamp was used to test B cell HVA calcium channel characteristics (including calcium current density, I-V curve, activation curve, inactivation curve) in both groups of SGA and AGA. Capacitance variation which could reflect single B cell secretion was also recorded by HEKA double patch clamp 
system after cell being stimulated by giving a sine wave stimulus. Results. SGA neonatal rats showed much lower values of the HVA calcium current density in pancreatic islet $\mathrm{B}$ cells than that of group AGA $(-5.94 \pm 0.53$ versus $-8.05 \pm 0.73 \mathrm{pA} / \mathrm{pF}$ obtained from $-40 \mathrm{mV}$ to $0 \mathrm{mV}$ depolarization, $P<.05 ;-6.05 \pm 0.93$ versus $-10.69 \pm$ $1.48 \mathrm{pA} / \mathrm{pF}$ obtained from I-V curve peak value, $P<.05)$. There were no statistical differences found among the other calcium channel characteristics of the two groups. The membrane capacitance increment of B cells was significantly smaller in SGA rat $(4.33 \pm 1.14 \mathrm{fF} / \mathrm{Pf})$ compared with that of AGA $(12.70 \pm 2.51 \mathrm{fF} / \mathrm{Pf})$ after excluding the influence of whole cell membrane capacitance deviation $(P<.05)$. Conclusion. Low HVA calcium current density on B cell membrane, which may lead to changes in calcium signalling, plays an important role in decrease of pancreatic islet B cell secretion in SGA neonatal rat caused by intrauterine malnourishment.

\section{Insulin Resistance and Metabolic Syndrome in Children with Obesity}

\author{
Yonglan Huang, Junqing Huang, Wenchun Lin, \\ Xiuzhen $\mathrm{Li}$, and $\mathrm{Li}$ Liu
}

\section{Department of Endocrinology and Metabolism, Guangzhou Women and Children's Medical Center, Guangzhou, 510623, China}

Objective. To observe the rates of occurrence of insulin resistance (IR) and metabolic syndrome (MS) and the association between IR and MS in a group of Chinese obese children. Methods. Oral Glucose Tolerance Tests (OGTT) were done and serum insulin measured in 76 children (49 males and 27 females) with obesity aged between 3 and 14 years old. Fasting blood sample was also obtained to measure total cholesterol, triglycerides, HDL-C, LDL-C, uric acid, liver function, and androgen in serum. Insulin resistance was defined as fasting levels of insulin higher than $15 \mathrm{mU} / \mathrm{ml}$, insulin peak (post-OGTT) levels higher than $150 \mathrm{mU} / \mathrm{ml}$, and/or insulin level higher than $75 \mathrm{mU} / \mathrm{ml}$ at $120 \mathrm{~min}$ of OGTT. Metabolic syndrome was diagnosed if three or more of the following criteria were present: obesity, dyslipidemia, impaired glucose tolerance and systolic or diastolic blood pressure above the 95th percentile. Results. In the 76 obese children, $68.4 \%$ of them had IR, $27.6 \%$ had impaired glucose tolerance or type II diabetes, $61.8 \%$ had dyslipidemia, $14.5 \%$ had hypertension, $28.9 \%$ had hyperuricemia, and $26.3 \%$ had increased ALT. Hyperandrogenism was found in 6 out of the 27 female children. $32.9 \%$ met the criteria of MS. The rates of impaired glucose tolerance, dyslipidemia, and MS were $34.6 \%, 73.1 \%$, and $44.2 \%$, respectively, in children with IR, which are significantly higher than those without IR $(P<.05, .01$, and .01 , resp. $)$. Out of the 20 cases with delayed or absent insulin responsive peak, 13 had impaired glucose tolerance and 1 was diagnosed with Type II diabetes. Conclusion. Insulin resistance and the Metabolic Syndrome were very common in children with obesity.
The occurrence of MS is strongly and positively related to IR.

\section{Parental Views on Sedentary Lifestyles in Overweight Children}

\author{
Madarina Julia ${ }^{1}$ and Emy Huriyati ${ }^{2}$ \\ ${ }^{1}$ Department of Child Health, Faculty of Medicine, \\ Gadjah Mada University/Dr Sardjito Hospital, Yogyakarta, \\ Indonesia \\ ${ }^{2}$ Department of Nutrition and Health, Faculty of Medicine, \\ Gadjah Mada University, Yogyakarta, Indonesia
}

Sedentary lifestyle is recognized as one of the risk factors for obesity in children and, hence, should be discouraged. Success in the promotion of active lifestyle in obese children is dependent on how parents view the problem. The objective of the study was to assess parental views on sedentary lifestyle in obese children in Yogyakarta, Indonesia. Subjects were 248 overweight children aged from 7 to 13 years old with their parents. The children's body weights and heights were measured in a screening for obesity in primary school children in Yogyakarta performed in the same year (2005). Overweight in children was defined as BMI-forage at the 85th percentile of the CDC 2000 growth chart or more. Data on parental body weights and heights, as well as questionnaires on parental views, were collected by visiting the households of the respondents. Parental overweight was defined as BMI of 25 or more. Information on physical activity was estimated from multiple 24-hour recalls (3 working days and one holidays) obtained from both the children and their parents. Data were obtained from $240(96.8 \%)$ fathers and $247(99.6 \%)$ mothers; 239 (96.4\%) children had complete data of both parents. The prevalence of overweight was $38.3 \%$ in fathers and $45.7 \%$ in mothers: $46.4 \%$ had one overweight parent and 18.8 had both parents overweight. Regarding the posture of their children, $90(36.3 \%)$ parents did not view their children as too fat. Only $71(28.6 \%)$ parents thought their children need to reduce weight, $24(33.8 \%)$ of them thought their children need to increase their physical activity. There was no association between parental overweight status and their view of their children's posture. Mean (SD) time spent on watching television was $2.9(0.9) \mathrm{hr} /$ day. Selecting $88(35.5 \%)$ parents who thought their children spent moderate time to watch TV resulted in mean (SD) time for watching TV of $2.8(0.8) \mathrm{hr} /$ day. Mean (SD) time spent on overall activities performed sitting was 11.4 (0.9) hr/day, while mean (SD) time spent on a more active life, for example, sport, walking, and cycling was 2.6 (0.9) hr/day. Median (Q1, Q3) time spent on sport was only $0.4(0.2,0.7) \mathrm{hr} /$ day. Selecting $113(45.6 \%)$ parents who thought their children spent moderate time for sports resulted in median $(\mathrm{Q} 1, \mathrm{Q} 3)$ time for sport of $0.6(0.3$, $0.8) \mathrm{hr} /$ day. We concluded that most parents were unaware of problems of sedentary lifestyles in their overweight children. 


\section{Effects of Exercise Training on Adiposity, Metabolic Parameters, and Adipokines in Obese Young Adults}

\author{
Eun Young Kim, ${ }^{1}$ Gook Chan Jang, ${ }^{1}$ \\ Kyung Rae Moon, ${ }^{1}$ and Kyung Hee $\mathrm{Yi}^{2}$ \\ ${ }^{1}$ Department of Pediatrics, Chosun Medical School \\ ${ }^{2}$ Department of Pediatrics, Wonkwang University Sanbon \\ Medical Center
}

Objective. Adipokines secreted by adipose tissue are suggested to play a role in the development of obesity-related complications. Regular exercise has been shown to reduce the risk of metabolic complications in obese subjects. The aim of this study was to investigate the effect of exercise training on body compositions, metabolic parameters, and adipokines in obese young adults. Methods. A total of 25 young adults (13 obese and 12 nonobese) were recruited in the public health center of Chosun University from January 1st to June 30th 2009. 8-week exercise training program (60-90 min/day, 3 days/week) was performed in all participants. We measured body compositions, serum lipid profiles, glucose, insulin, leptin, and adiponectin before and after exercise in control and obese young adults. Results. (1) Body weight (BW), BMI, body fat percentage, and visceral fat percentage were significantly decreased in control and obese group after exercise $(P<.05)$. (2) In metabolic biomarkers, serum insulin, HOMA-IR, AST, and ALT levels were decreased in obese group than control group after exercise $(P<.05)$. (3) Serum leptin concentrations were significantly increased in obese than control group before exercise $(P<.05)$. (4) Serum leptin concentrations were significantly decreased after exercise in obese group after exercise $(P<.05)$. (5) Leptin/adiponectin (L/A) ratio was significantly increased in obese than control group and decreased in obese and control group after exercise. (6) Changes of leptin concentrations after exercise correlated with changes of BMI $(r=0.471, P=.013)$, adiponectin $(r=0.430, P=.025)$, and leptin/adiponectin ratio $(r=0.807, P<.0001)$. Conclusion. Body composition, leptin, and L/A ratio were significantly decreased in the obese group after exercise. After exercise, changes of leptin concentrations correlated with changes of BMI and adiponectin.

\section{The Relation of the RBP4 Levels and Insulin Resistance in Obese Children}

\section{Se Young Kim}

\section{Bundang Jesaeng General Hospital, DMC}

Objectives. RBP4 has been proposed to be a marker for diabetes. There are multiple mechanisms described by which RBP4 can effect changes in glucose metabolism. We examined the serum RBP4 levels and insulin resistance in obese children. Methods. We compared the serum RBP4 levels in obese group (BMI > 95 percentile for age, sex) and control group (measured with ELISA kit). In both groups, we measured fasting glucose, fasting insulin, RBP4, AST/ALT, lipid panel, and insulin resistance by HOMA-IR. Then we examined the correlation the serum RBP4 levels and insulin resistance in the obese group. Results. 20 obese children and 30 controls were enrolled. In both groups, the serum RBP4 levels were not different. In the obese group, serum RBP4 levels correlated positively with total cholesterol, HDL, LDL, and TG concentrations. But that was not correlated with HOMA-IR. Conclusion. Considerable work is required to prove the value of the serum RBP4 levels in obese children. Further methods other than ELISA would be required.

\section{Timing of Adiposity Rebound May Not Be Related to Body Weight Gain during Infancy and Is Late by Breast-Feeding in Early Infancy}

\author{
Satomi Koyama, ${ }^{1}$ Go Ichikawa, ${ }^{1}$ Yuzuru Yamazaki, ${ }^{1}$ \\ Megumi Kojima, ${ }^{1}$ Naoto Shimura, ${ }^{1}$ \\ Toshimi Sairenchi, ${ }^{2}$ and Osamu Arisaka ${ }^{1}$ \\ ${ }^{1}$ Department of Pediatrics, \\ ${ }^{2}$ Department of Public Health, Dokkyo Medical University, \\ Japan
}

Background. The age of adiposity rebound (AR), when body mass index (BMI) starts to rise after infancy, is thought to be a predictor of obesity in later life. We have already reported that children who exhibited an earlier AR have a higher BMI and atherogenic metabolic status at 12 years old. It has also been reported that weight gain during early infancy predisposes children to future obesity, and breastfeeding is associated with a decreased incidence of obesity in childhood. Objectives. The aim of the study was to investigate whether increments of weight gain and the type of nutrition in early infancy are related to the timing of AR. Methods. A total of 271 children (147 boys and 124 girls) in one community were enrolled in the study. Serial measurements of BMI from age 4 months to 12 years old were carried out prospectively to determine the age of AR. Body weight increments were measured in three periods (birth $-4 \mathrm{~m}$, $4 \mathrm{~m}-8 \mathrm{~m}$, and $8 \mathrm{~m}-12 \mathrm{~m}$ ). At 10 years of age, serum lipid and lipoprotein levels and blood pressures were measured. We also questioned parents about nutrition in early infancy and categorized this as breast-feeding, bottle-feeding, or mixed feeding. Results. No significant relationships were observed between weight gain during any period of infancy and the age of AR. Weight gain between birth and 4 months of age was positively correlated only with BMI of 7 years of age. Only $15 \%$ of children with an obesity index $\geq 20 \%$ at 12 years old were obese in infancy. Breast-feeding in infancy was associated with a later age of AR. BMI through childhood was lower for infants fed by breast-feeding compared to those fed by bottle-feeding. Breast-feeding tended to decrease BMI and the triglyceride level and increase HDL-C level at 10 years old compared to bottle-feeding but the difference was not significant. Conclusion. Contrary to expectation it appeared 
that body weight gain during infancy was not associated with the timing of AR, suggesting that infantile obesity does not relate to childhood obesity. Breast-feeding seems to have a favorable affect on prevention of future metabolic syndrome.

\section{Independent Relationships of Obesity and Insulin Resistance with Serum Proinsulin Level in Prepubertal Children with Normal Glucose Tolerance}

Young Ah Lee, ${ }^{1}$ Jae Ho Yoo ${ }^{2}$ Jae Hyun $\mathrm{Kim}^{3}$ Sun Hee Lee, ${ }^{4}$ Ji Hyun Kim, ${ }^{5}$ Han Hyuk Lim, ${ }^{6}$ Min Jae Kang, ${ }^{1}$ Shin Mi Kim, ${ }^{1}$ Hye Rim Chung, ${ }^{7}$ Choong Ho Shin, ${ }^{1}$ and Sei Won Yang ${ }^{1}$

\section{${ }^{1}$ Department of Pediatrics, Seoul National University College of Medicine \\ ${ }^{2}$ Department of Pediatrics, Dong-A University \\ Medical Center \\ ${ }^{3}$ Department of Pediatrics, IIsan Paik Hospital \\ ${ }^{4}$ Department of Pediatrics, Pusan Paik Hospital \\ ${ }^{5}$ Department of Pediatrics, Pohang St. Mary's Hospital \\ ${ }^{6}$ Department of Pediatrics, Chungnam National University College of Medicine \\ ${ }^{7}$ National University Bundang Hospital}

Objectives. We compared the fasting serum proinsulin levels in lean, overweight, and obese prepubertal children with normal glucose tolerance (NGT). We also evaluated the relationship between fasting proinsulin level and indices of insulin resistance (IR). Methods. One hundred nine prepubertal children (mean age, 8.6 years) with NGT were included. The indices of IR included the homeostasis model assessment of IR (HOMA-IR) and adiponectin level. We recorded the presence of 1 or more of the following metabolic derangements: triglycerides $\geq 150 \mathrm{mg} / \mathrm{dl}$, HDL cholesterol $<35 \mathrm{mg} / \mathrm{dl}$, and hypertension. Results. Fasting proinsulin levels significantly increased with BMI category from lean $(n=52,7.22 \pm 3.01 \mathrm{pmol} / \mathrm{l})$ to overweight $(n=14,12.31 \pm 2.91 \mathrm{pmol} / \mathrm{l})$ to obese $(n=43$, $16.51 \pm 7.27 \mathrm{pmol} / \mathrm{l})(P<.001)$, after controlling for HOMA-IR. The ratio of the fasting levels of proinsulin to insulin did not differ significantly between the 3 groups. Both BMI z-score and HOMA-IR were significant independent factors related to the fasting proinsulin level $(P<.001$ for each). A significant inverse association was found between fasting proinsulin level and adiponectin level $(r=-0.464, P<.001)$. Children with NGT who had at least 1 metabolic derangement had higher proinsulin levels than those without metabolic derangement. Conclusions. Obesity itself or obesity-related IR may independently impose b-cell overload on prepubertal children with NGT, leading to hyperproinsulinemia without causing failure to convert proinsulin to insulin when some degree of IR and metabolic derangement appears.

\section{Association of FTO, Pyrophosphatase/Phosphodiesterase 1, Adiponectin Gene Polymorphism with Obesity and Related Parameters in Chinese Han Children and Adolescents}

\author{
Feihong Luo, Lingfeng Cao, Yi Yang, Di Jingzhi, \\ Ruoqian Cheng, and Shuixian Shen
}

The Department of Pediatric Endocrinology and
Metabolism, Children's Hospital of Fudan University,
Shanghai 201102, China

Objective. The single nucleotide polymorphism rs9939609, rs1421085 of FTO gene, K121Q polymorphism of ectonucleotide pyrophosphatase/phosphodiesterase 1 gene (ENPP1), and SNP276 polymorphism of adiponectin gene (AMP1) have been studied in relation to obesity, insulin resistance and type 2 diabetes in several populations with discrepant results. We therefore investigated the role of the above SNP on obesity and relative trails in Chinese Han children and adolescents. Methods. 458 obese/overweights children and adolescents and 231 normal weight control aged 6-18 years were recruited. Body height and weight were measured, and body mass index (BMI) was calculated. Serum fasting plasm glucose (FPG), fasting insulin (FIns), triglycerid (TG), and cholesterol (TC) were measured; HOMA-IR and QUICKI were calculated. Genomic DNA was extracted form peripheral blood; Taqman-MGB probe was used to determine the polymorphism. Obesity was evaluated using IOTF BMI standard. Results. (1) The fasting plasma glucose, insulin, TG, HOMA-IR were significantly higher than those in normal control group. (2) BMI existed significant statistical difference between the rs1421085 TC +CC, rs9939609 TA +AA genotypes when comparing with their wild TT genotype of FTO gene (rs9939609: $P=$.0003; rs1421085: $P=.0005)$. However, FPG, Fins, TG, TC, HOMA-IR, and QUICKI showed no significant statistical difference between the rs1421085 TC +CC, rs9939609 TA +AA genotypes and their wild TT genotype. (3) The G allele frequency of SNP276 of AMP1 gene was $69.7 \%$ which existed no significant statistical difference when compared with obesity (allele frequency $71.4 \%$ ) and overweight (allele frequency $72.5 \%)$ group $(P=.39)$. (4) The frequency of $\mathrm{K}$ allele was $89.4 \%$ and $\mathrm{Q}$ allele frequency was $10.6 \%$ in the obese group, $\mathrm{K}$ allele was $90.7 \%$, and Q allele frequency was $9.3 \%$ in the overweight group, and $\mathrm{K}$ allele was $97.9 \%$ and Q allele frequency was $12.1 \%$ in normal control subject; there were no statistic differences among the three groups $(P=.4171)$. Conclusion. We report that FTO rs9939609, rs1421085 SNP are associated with obesity and BMI in Chinese Han children and adolescents, but failed to find any significant statistical association among the SNPs and obesity-related metabolic parameters. ENPP1 K121Q and APM1 gene SNP276 polymorphism may play minor roles in the genetic etiology of Chinese children obesity. 


\section{Diabetes in 6 Patients of Beta-Thalassemia Major}

\author{
Zhe Meng, Li-yang Liang, Xiang-yang Luo, \\ Dan-yang Cen, and Dong-fang Li
}

Department of Pediatrics, Sun Yat-Sen Memorial Hospital, Sun Yat-Sen University GuangZhou City 510120, China

Objective. The aim of this study was investigation of clinical features, pathogenesis, laboratory examination and early treatment of diabetes mellitus in patients with betathalassemia major. Methods. Diabetes mellitus was observed in 6 of 53 patients with beta-thalassaemia major attending Sun Yat-sen memorial hospital. In this retrospective analysis study, patients were examined to determine their pubertal status and the standard deviation score for height for evaluation of short stature. Liver enzymes, liver size and indicators of iron overload (serum ferritin, total units of blood transfused, splenic size, pancreas MRI) were recorded. A fasting blood glucose, insulin level, C peptide and HbAlc were performed. Evidence for diabetes mellitus was based on American Diabetes Association and World Health Organization criteria. Serum levels of calcium, phosphorous, thyroidstimulating hormone, totle thyroxin, luteinizing hormone, follicular-stimulating hormone, estradiol, testosterone, cortisol and ACTH were measured. Results. The average age in the 6 patients was 13.36 years (11-18 years). Two patients, at onset of clinical diabetes, presented with an asymptomatic glycosuria and four with polydipsia, polyuria, weight loss and ketoacidosis. The induced factors were abscess, chest wall abscess, pneumonia and heart failure. Serum ferritin was in the range of $4800-7148 \mathrm{ug} / \mathrm{L}$; Fasting blood glucose was in the range of $8.9-43.8 \mathrm{mmol} / \mathrm{L}$, HbAlc was in the range of $8.9-16.8 \% .2$ patients had detected high insulin level $(>20 \mathrm{mu} / \mathrm{L})$ suggesting insulin resistance. 4 cases of ketoacidosis were detected insulin level $<2 \mathrm{mu} / \mathrm{L}, \mathrm{C}$ peptide mean $24 \mathrm{pmol} / \mathrm{L}$, suggesting lack of insulin secretion. Pancreas MRI showed the signal to reduce, which was related with iron deposition. The puberty stage of 6 patients was in Tanner 1 to Tanner 2. 6 patients were all short stature, 4 cases of hypothyroidism, 2 cases of GHD. Adrenal and kidney function was normal, mild liver function abnormalities ( 2 cases) and abnormal heart function (1 case) were detected. All patients treated with transfusion, deferoxamine, exercise, diet control, Glucobay and insulin. Comprehensive therapy was good. Conclusions. Despite therapy with deferoxamine to treat iron overload, the risk of secondary endocrine dysfunction remained high. Diabetes in patients of beta-thalassemia major were often accompanied by other endocrine organ damaged, which occurs with iron deposition. The pathogenesis of diabetes in beta-thalassemia major may be similar to the development of type 2 diabetes. Early diabetes, it was insulin resistance, and finally lack of insulin secretion induced to insulin dependent diabetes mellitus.

\section{Insulin Resistance of Obese Children and Adolescents with Type 2 DM Family History}

\author{
Oh Yeon Joung, Nam Hyo Kyoung, Park Sang Hee, \\ Son Chang Sung, and Lee Kee-hyoung
}

\author{
Department of Pediatrics, College of Medicine, \\ Korea University
}

Objectives. The current worldwide increase of type 2 diabetes mellitus (T2DM) coincides with the increase of obesity. Also, family history of T2DM (FHD) is strongly associated with T2DM in childen as well as adults. We evaluated insulin resistance of obese children with FHD compared with obese children without FHD. Methods. The study included 66 children and adolescents aged 8-15 years who were divided into two groups according to FHD: 30 obese adolescents with FHD and 36 obese adolescents without FHD. Anthropometric measurements were obtained. Blood samples were collected and fasting plasma glucose (FPG), serum lipids, fasting insulin (FI) were assayed. All subjects were performed oral glucose tolerance test. Results. HOMA-IR and FI levels of obese children with FHD showed significantly higher than that of obese children without FHD (HOMA-IR:5.5 \pm $3.4 \mathrm{uIU} / \mathrm{mL}^{*} \mathrm{mmol} / \mathrm{L}$ versus $4.0 \pm 1.8 \mathrm{uIU} / \mathrm{mL}^{*} \mathrm{mmol} / \mathrm{L}, P<$ $.05)$. In obese children with FHD, the frequency of impaired glucose tolerance was higher than that of obese children without FHD (23.3\% versus 5.6\%). Conclusions. In obese children, especially with T2DM family history, evaluatation of diabetes or IGT is required.

\section{Relation between Acanthosis Nigricans and Family History of Type 2 Diabetes Mellitus in Overweight and Obese Children}

\author{
Vivekenanda Pateda, Adrian Umboh, \\ Kristellina Tirtamulia, and Frecillia Regina \\ Endocrinology Division, Pediatric Department, \\ Medical Faculty Sam Ratulangi University/Kandou \\ General Hospital Manado, and Pediatric Endocrinology \\ Chapter, Indonesian Pediatric Society
}

Introduction. With the rise of obesity in pediatric population recently, the type 2 Diabetes Mellitus (T2DM) prevalence is also increasing. Acanthosis Nigricans (AN) is believed to be a good clinical predictor of hyperinsulinemia, the T2DM precursor. Previous studies showed that there were higher AN prevalence in overweight and obese children with family history of T2DM. Objective. To investigate the relation between AN and family history of T2DM in overweight and obese children. Methods. This was a cross sectional study conducted in junior high school children at Wenang District, Manado. Student $t$-test was used to analyze one hundred and two children with overweight and obese that has been screened for AN and questioned about T2DM family history. Results. AN was higher in obese (69\%) rather than overweight (44\%). T2DM family history was found in $30 \%$ obese and $31 \%$ overweight children 
with AN. Conclusion. No significant relation between AN and family history of T2DM in overweight and obese children.

\section{The Relationship between the Concentration of IL-6 in Fat Tissue and Metabolic Syndrome with Cardiovascular Risk Factors in Young Rats}

\section{Bao PengLi, Liu GeLi, Yang QingYan, and Zheng RongXiu \\ Pediatric Department, General Hospital Of Tianjin Medical University}

Objective. To discuss whether the concentration of the IL-6 changes in the early stage of the Metabolic Syndrome (MS). To investigate the correlation between the concentration of IL-6 and the risk factors of the cardiovascular disease in MS models. Methods. 40 weaned rats, including 20 males and 20 females, divided into 3 groups randomly. MS models were established by high fat salt diet, obesity and lipid metabolism disturbance models were established by high fat diet and the control group were given common diet. After 4 weeks feeding, the weight, length, waist circumference (WC) and blood pressure (BP) were measured of the young rats, concentration of the lipid profiles like HDL, LDL, TG, TC and the fasting blood glucose (FBG), fasting blood insulin (FIN) were detected at the same time. Then the fat tissue samples were weighed and the concentration of the IL- 6 was measured in the fat tissue by ELISA. After that, calculating if there was statistical significance between the three groups. To investigate the correlation between the concentration of IL-6 and the associate factors, just like HDL, LDL, FIN, TC, TG, FBG, HOMA-IR, the weight of the fat tissue samples and the body. Finally, calculating the degree of the correlation. Results. The concentration of the IL-6 in the fat tissue from the MS group, obesity with lipid disturbance group were higher than that of the control group $(P<.05)$. The level of IL-6 in MS group was higher than that in obesity with lipid disturbance group $(P<.05)$. The concentration of the IL-6 was significantly correlated with LDL, FIN, HOMAIR, TC, TG, HDL, the weight of the fat tissue and body in the MS group; IL-6 in the obesity group was significantly correlated with TC, HDL, TG, W, the weight of fat tissue Conclusion. The concentration of the IL-6 in the MS and obesity and lipid disturbance group increased indicating IL-6 participates in the disturbance of lipid metabolism and obesity. The difference between the MS group and obesity with lipid disturbance group also shows that IL-6 participates in the MS process also through the other ways besides obesity and the lipid disturbance. The level of IL-6 in the obesity with lipid disturbance group was correlated with TC, LDL, TG, W and the weight of the fat tissue, further indicating that IL-6 participates in the development of obesity and disorders of lipid metabolism. IL- 6 could be an index in prediction the early pathological process of the cardiovascular disease

\section{Impact of Exercise on Hsc-Rp Level in Obesity Adolescents}

\author{
Eka Agustia Rini, Susetyo
}

Pediatric Endocrinology Sub Division, Medical Faculty of Andalas University Padang, Indonesia

Background. The incidence of obesity in children and adolescents is increasing. Obesity plays an important role in the occurrence of cardiovascular disease. The high level of hsC$\mathrm{RP}$ is one of the strong indicators of cardiovascular events in obese people. Regular exercise would reduce the degree of obesity and hsC-RP level and eventually decrease the risk of future cardiovascular disease. Objective. To evaluate the impact of regular physical exercise on hsC-RP levels in adolescents with obesity. Methods. Experimental study was conducted on 40 obesity adolescents from November to December 2009. Samples were divided into physical exercise group who performed physical training for one month and control group. Level of hsC-RP was measured before and after the study done. Data were analyzed with chi square and $t$-test with a significance level of $(P<.05)$. Results. The mean of hsC-RP level in the beginning of the study was higher in the physical exercise group than the control $(4.35 \mathrm{mg} / \mathrm{dL},+3.10$ versus $2.85 \mathrm{mg} / \mathrm{dL},+3.10)$. At the end of the study, BMI decreased in 18 samples (90\%) from exercise group and two samples (10\%) from the control $(P=.000)$. There was a decrease in hsC$\mathrm{RP}$ level in the exercise group $(2.47 \mathrm{mg} / \mathrm{dL},+2,35)$ and increase in the control $(3.32 \mathrm{mg} / \mathrm{dL},+2.57)(P=.02)$. Conclusion. There was a significance decrease in hsC-RP level after physical exercise in obese adolescents compared to the control group.

\section{Analysis of Brain Leptin in Young Rat with Metabolic Syndrome}

\section{Zheng Rongxiu, Liu Geli, and Yang Linjie \\ Tianjin Medical University General Hospital}

Objective. To investigate the effects of food high in fat and salt on the level of hypothalamic leptin, body fat distribution, blood pressure, and gluco-lipid metabolism. To analyze the correlation between hypothalamic leptin and other indices, then evaluate the role of leptin in the development of metabolic syndrome. Methods. Forty young SD rats, half males and half females, were randomly divided into three groups: high fat and high salt group (14 cases), high fat group, (14 cases) and control group (12 cases). After four weeks, OGTT, hypothalamic leptin, blood pressure, blood lipid, cortisol, body fat distribution, blood glucose, and insulin were measured. Results. In high fat and high salt group, body weight, waist circumference, and visceral fat weight were significantly higher than in control group $(P<.05)$. In high fat group, body weight and visceral fat weight were significantly higher than in control group $(P<.05)$. In high fat and high salt group, blood glucose and serum insulin at fasting, 60 minutes, 120 minutes, 
and 180 minutes were significantly higher than in control group $(P<.05)$. In high fat group, blood glucose at 60 minutes was significantly higher than in control group. In high fat and high salt group, systolic pressure, TC, TG, LDL$\mathrm{C}$ were significantly higher than in control group $(P<$ $.05)$, while HDL-C were significantly lower than control group $(P<.05)$. In high fat and high salt group, brain leptin was significantly lower than in high fat group and control group $(P<.05)$. In high fat group, brain leptin was significantly lower than in control group. In high fat and high salt group, brain leptin levels were negatively correlated with visceral fat weight $(r=-0.453)$, fasting blood glucoseserum $(r=-0.286)$, insulin $(r=-0.566)$, systolic pressure $(r=-0.472)$, and TG $(r=-0.511)$, while positively correlated with HDL-C $(r=0.38)$. In high fat group, brain leptin levels were negatively correlated with visceral fat weight $(r=-0.179)$, insulin $(r=-0.464)$, blood pressure $(r=-0.286)$, and TG $(r=-0.607)$, while positively correlated with HDL-C $(r=0.643)$. Conclusion. A model of metabolic syndrome was made via food high in fat and salt. Brain leptin levels were negatively correlated with visceral fat weight, fasting blood glucose, serum insulin, systolic pressure and TG, while positively correlated with HDL-C. Metabolic syndrome is the result affected by many fators. Brain leptin may play a role in metabolic syndrome via affecting body fat distribution, blood pressure, and glucolipid metabolization.

\section{The Relationship between Serum High-Sensitivity C-Reactive Protein and the Insulin-Like Growth Factor Binding Protein-1 in Obese Children}

\author{
Bing Sun, Chunxiu Gong, Xiaoqun Zhang, \\ and Jianyun Jin
}

\section{Beijing Children's Hospital Affiliated Of Capital University Of Medical Sciences}

Objective. The present study aimed to understand the possible correlation between serum high-sensitivity C-reactive protein, the insulin-like growth factor binding protein-1, and some related factors of obese children. Furthermore, we investigated the roles of hs-CRP and IGFBP-1 in children in predicting impaired glucose metabolism. Methods. Fifty-nine obese children and thirty-nine nonobese healthy controls (group 3) were enrolled into this study. The obese individuals were subdivided into two subgroups according to the results of OGTT: sixteen obese subjects with impaired glycometabolism (group 1) and forty-three obese subjects without IGR (group 2). The levels of serum parameters including hs-CRP, IGFBP-1, glucose, lipid, insulin, Cpeptide, HOMA- $\alpha$, HOMA- $\beta$, and so forth were determined. SPSS 11.0 was used for statistical analysis. Results. There was no obvious difference among the three groups in age. The fasting blood glucose (FBG), fasting insulin level (FIns), fasting C-P level, HOMA- $\alpha$, and HOMA $\beta$ of the obese children especially in the IGR group were much higher than those of the normal ones. There was an increase of serum hs-CRP level in obese children. The median in group 1 was $1.35 \pm 0.10 \mathrm{mg} / \mathrm{L}$, in group 2 was $2.80 \pm$ $0.01 \mathrm{mg} / \mathrm{L}$; the level of control group was $2.80 \pm 0.01 \mathrm{mg} / \mathrm{L}$. There was a significant decrease of serum IGFBP-1 level in obese children. The average of group 1 and group 2 was $4.52 \pm 5.31 \mathrm{ng} / \mathrm{mL}$ and $7.77 \pm 7.88 \mathrm{ng} / \mathrm{mL}$, respectivly; the level of control group was $31.12 \pm 23.13 \mathrm{ng} / \mathrm{mL}$. This indicated that the changes of hs-CRP and IGFBP-1 might happen before the changes of IGR. However, there was no significant difference of hs-CRP and IGFBP-1 between these two groups in the obese subjects with or without IGR. There was a significant difference of $\operatorname{HOMA}-\alpha$, not only between groups, but also between the obese subjects with and without IGR. For the nonobese healthy control group, IGFBP-1 had more primary correlation with FBG, Fins, and HOMA- $\alpha$ than with age, BMI, IGF-1, and other factors. Multiple linear regression analysis showed that FBG, FIns, and HOMA- $\alpha$ were enrolled in the regression equation. Pearson correlation analysis showed that there was no significant correlation between serum hs-CRP and all parameters in obese children. On the contrary, however, it showed that there was a moderate correlation between serum IGFBP-1 and many parameters (such as BMI, fasting insulin level, 2-hour insulin level, fasting C-peptide level, 2hour C-peptide level, WC, SBP, HOMA- $\alpha$, and $\mathrm{HOMA} \beta$ ) in obese children. Conclusion. Along with the changes in serum hs-CRP and IGFBP-1, there might be a chronic lowgrade inflammation and potential insulin resistance in obese children prior to the presence of IGR. To some extent, the situation of low-level serum IGFBP-1 and high-level hsCRP reflected an increased morbidity risk of IGR in obese children, which indicated that this might be an alternative screening method for OGTT. Independently, low IGFBP-1 is a more predictive factor of the presence of IGR and metabolic syndrome than high-level hs-CRP. There was a significant difference of FIns level between group 1 and group 2, but not for $2 \mathrm{hIns}$. Therefore, it can be concluded that IGFBP-1 is correlated primarily to the level of $2 \mathrm{hIns}$, which reflects that hyperinsulinemia may be a compensatory status during the early stage of IGR. Although CRP is an indicator of the inflammation, it is also sensitive to many other factors such as infection as well as obesity. Compares to CRP, IGFBP-1 is relatively more stable and highly sensitive to glucose metabolism. Nevertheless, as the cost of IGFBP-1 is much more expensive than that of hs-CRP, therefore further research is expected.

\section{Relationship between Retinol-Binding Protein 4, Obesity, and Insulin Resistance}

\section{Xian Wu and Yu Yang \\ JiangXi Children's Hospital}

Objective. Retinol-Binding Protein 4 (RBP-4) is a newly discovered Adipocytokine. There are limited and controversial studies concerning the relationships between RBP-4, body mass index (BMI), and insulin resistance in obese humans and especially in children. This study aims to 
find out the relationship between RBP-4 levels and obesity and insulin resistance. Method. Choose 71 inpatients of endocrinology department of JiangXi children's hospital during Mar 2008 to Mar 2009. There were 42 obese children, among which 19 had substantial weight loss after 6 months. Measuring weight, height, waistline, blood pressure, and determine the serum RBP-4, fasting blood sugar, blood fat and insulin levels, and calculate insulin resistance index homeostasis model assessment (HOMA-IR), analysis the correlation between RBP-4 levels, BMI, fasting glucose, and HOMA-IR. Results. Compared with the normal body weight children, obese children had significantly $(P<.01)$ higher RBP4 concentrations. In multiple linear regression analyses adjusted to age, gender, and pubertal stage, RBP4 was significantly correlated to BMI and HOMA-IR. Conclusion. Obese children had a higher serum RBP4 concentrations and RBP4 levels had positive correlation with BMI and HOMA-IR.

\section{Neonatal Overfeeding Exacerbates High-Fat Diet-Induced Metabolic Perturbation}

Xiao Qiu Xiao, ${ }^{1}$ Yong Song, ${ }^{1}$ Maria Glavas, ${ }^{2}$ Melissa Kirigiti, ${ }^{2}$ Chun Yan Lim, ${ }^{3}$ Michelle Yu-Fah, ${ }^{3}$ Guanghou Shui, ${ }^{4}$ Markus Wenk, ${ }^{4}$ Weiping Han, ${ }^{3}$ and Kevin Grove ${ }^{2}$

\section{${ }^{1}$ Chongqing Medical University \\ ${ }^{2}$ Oregon Health \& Science University \\ ${ }^{3}$ Laboratory of Metabolic Medicine, \\ Singapore Bioimaging Consortium \\ ${ }^{4}$ Department of Biochemistry, \\ National University of Singapore}

Objectives. To investigate whether neonatal overfeeding would permanently increase susceptibility to obesity, insulin resistance, and sensitivity to high fat diet (HFD) and potential molecular mechanisms. Methods. Pups were raised in either 3pups/litter (chronic postnatal overfeeding, CPO) or 10pups/litter (control, CTR). After weaning, a subset of mice was placed onto HFD. Epididymal white adipose tissue (WAT) and skeletal muscle (SKM) were harvested to assess inflammatory and lipogenesis pathways through approaches of histology, biochemistry, transcript, and protein expression. Results. Under HFD, CPO mice exhibited accelerated body weight gain, rapid development of obesity and insulin resistance, and more severe impairment in GTT compared with CTR mice. Insulin signal pathways and insulin response impaired more severely in WAT and SKM from CPO-HFD mice. Increased F4/80 immunohistochemical activities, increased gene expression of inflammatory mediators, and sustained activation of inflammatory pathway were observed in WAT from CPO-HFD. Lipogenesis was coordinately inhibited in WAT while activated in SKM in the presence of HFD, which in turn resulted into excess intramyocellular lipid accumulation in SKM. Conclusion. Postnatal exposure to overnutrition leads to a more active inflammation and the oversupply of fat to SKM, which likely increases susceptibility to insulin resistance and exacerbates the detrimental outcome in response to HFD in adulthood.

\section{To Evaluate the Diagnostic Value of Combining Hepatic B-Ultrasound Imagine and Noninvasive Biochemical Markers such as Transaminases (ALT, AST) Type đó Procollagen N Peptide (Pḉó) in Nonalcoholic Fatty Liver Disease, and Screen Applicable Noninvasive Diagnostic Markers for Compensated NAFLD in Obese Children}

\author{
Xu Xiao-Hua, Fu Jun-Fen, Huang Ke, \\ Wang Chun-Lin, and Liang Li
}

\section{Department Of Pediatrics, The First People's Hospital Of Huzhou}

Objective. To evaluate the diagnostic value of combining Hepatic B-ultrasound imagine and noninvasive biochemical markers such as transaminases (ALT, AST), procollagen $\mathrm{N}$ peptide (Pcłó) in nonalcoholic fatty liver disease (NAFLD), and screen applicable non-invasive diagnostic markers for compensated NAFLD in obese children. Methods. In this study, 269 obese children and 30 healthy control group matched in age and sex were enrolled. We measured body mass index (BMI), waist and hip circumference, blood pressure, blood glucose, insulin, lipid profile, transaminases (ALT, AST), serum hepatic fibrosis markers hyaluronic acid(HA), type 4 collagen (CIV), procollagen $\mathrm{N}$ peptide (Pckó) and laminin (LN), and hepatic B-ultrasound in all participants. Oral glucose tolerance test and insulin-releasing test were performed. Three subgroups were selected according to the diagnostic criteria. Group 1: SOC (simple obese children), Group 2: NAFLD-1 (US fatty liver and normal transaminases), and Group 3: NAFLD-2 (US fatty liver and elevated transaminases). We also classified 269 obese children into three subgroups according to B-ultrasound imagine examination. Group 1: SOC (normal US liver and normal transaminases), Group 2: mild fatty liver, and Group 3:moderate and severe fatty liver. We compared body mass index (BMI), the ratio of waist and hip circumference, lipid profile and transaminases (ALT, AST), fasting blood glucose, insulin, serum hepatic fibrosis markers, HOMA score, and WBISI based on OGTT test in all participants and 30 healthy control. The binary Logistic regression analysis was applied between Group SOC and Group NAFLD. We evaluated and compared all the biochemical markers with the area under receiver-operating characteristics (ROC) curve. Results. (1) Compared with group SOC, the biochemical markers ALT, AST, TG, HA, PC, FINS, 2hINS, and WBISI were significantly increased in NAFLD-1 group, Compared with group NAFLD-1, the biochemical markers ALT, PC were significantly increased in NAFLD-2. (2) The level of ALT was significantly correlated with B-ultrasound examination, and its regression coefficient was $0.11(P=0)$. 
(3) The area under the receiver-operating characteristics (ROC) curve of ALT, AST, Pc was 0.88, 0.80, 0.70, which showed the most value in diagnosis of NAFLD in obese children. Conclusion. Combining Hepatic B-ultrasound and the biochemical markers ALT, AST, PC were most useful in diagnosis of NAFLD in obese children, which showed not only the degree of hepatic inflammation but also the degree of hepatic fibrosis.

\section{The Metabolic Disorders in Children with Abdominal and Nonabdominal Obesity}

\author{
Feng Xiong and Jie $\mathrm{Yu}$
}

\section{Children's Hospital Of Chongqing Medical University}

Objective. To investigate the characters of metabolic disorders in children with abdominal and nonabdominal obesity. Methods. 145 obese children and 80 healthy children were recruited. Height, body weight, waist circumference (WC), hip circumference, and blood pressure were measured and body mass index (BMI) were calculated. Obese children were divided into abdominal and nonabdominal obesity group according to BMI and WC. Blood lipids, serum glucose, and serum insulin were measured. Results. Abdominal obesity has an incidence of $87.59 \%$ (127 cases among 145 obese children), while nonabdominal obesity accounted for $12.41 \%$ (18 cases). Fasting blood glucose, total cholesterol (TC), triglyceride (TG), low density lipoprotein (LDL), and blood pressure in abdominal obesity children were higher than nonabdominal obesity and normal children $(P \pm$ $.05)$. Apart from TG, there were no significant differences between nonabdominal obesity and normal group. Among 127 abdominal obesity children, There were 77 cases of insulin resistance, 14 cases of impaired fasting glucose, 7 cases of damage carbohydrate tolerance, 24 cases of high TG Hyperlipemia, 13 cases of hypercholesterinemia, 10 cases of high LDL hyperlipidemia, and 15 cases of hypertension, only two cases of insulin resistance were observed in nonabdominal obesity. Conclusion. Abdominal obesity is the leading type of children obesity and is more susceptible to abnormality of metabolism than nonabdominal obesity.

\section{The Relationship between Evaluation of Glucose Intolerance in Obese Children and Positive Family History of Type 2 Diabetes Mellitus}

\section{Hui Yao, Shi-xiu Kang, and Juanjuan Ding \\ Wuhan Children's Hospital}

Objectives. There is an increasing trend in the prevalence of type 2 diabetes mellitus (DM2) in childhood and adolescence, while a positive family history of DM2, and obesity is the most important risk factor. The influence of family history and obesity on glucose intolerance was the aim of this study. Methods. A total of 65 children and adolescents aged $7-12$ years (mean $10.3 \pm 1.5$ years) were included in the study. All children and adolescents were divided into three groups according to positive family history of DM2 and obesity, and an oral glucose tolerance test (OGTT) was performed for all Prediabetes was defined as impaired fasting glucose tolerance (IGT) and/or impaired fasting glucose (IFG). Insulin secretion and insulin resistance were estimated using the insulinogenic index and the homeostatic model assessment for insulin resistance (HOMA-IR) and Matsuda index, respectively. Results. The prevalence of prediabetes was $15.4 \%$ in the whole group, while it was $25.0 \%$ in obese children who also had a positive family history of DM2. The frequency of hyperinsulinism was $36.9 \%$ in all groups, $50 \%$ in the group of obese children with a positive family history of DM2. Conclusions. The risk of prediabetes in children is highest in obese children who also had a positive family history of DM2. There is a need for a lifelong preventive program starting in childhood to avoid DM2 and decrease cardiovascular risk factors.

\section{Effect of Intrauterine Growth Retardation on Beta-Cell Regulating Factors: PDX-1, HNF-1 Alpha, and HNF-4 Alpha}

\author{
Zhang Jie, Xin Ying, and Li Shuqin
}

\section{Department of Pediatrics, Shengjing hospital affiliated to China medical university, Shenyang, China}

Objectives. Studies indicated that intrauterine growth retardation (IUGR) is related to the occurrence of diabetes mellitus. This study investigated the relationship of PDX-1, HNF-1 alpha, and HNF-4 alpha with beta cell to explore the possible role of these factors in the occurrence of diabetes mellitus. Methods. The murine model of IUGR was employed by low-protein diet. There is also temporal and spatial expression of PDX-1 in the pancreas as well as HNF-1 alpha and HNF-4 alpha in the liver of the newborn rats, 3week-old and 8-week-old rats, using Western-blot analysis, immno-histochemistry staining, and real-time PCR. Results. Western-blot analysis and immnohistochemistry staining revealed that signals specific for PDX-1 in the pancreas decreased in the newborn rats and 3-week-old rats and increased in the 8-week-old rats. There was significant difference between the IUGR and normal rats $(P<.05)$. Western-blot analysis and immnohistochemistry staining revealed that the expression level of HNF-1 alpha and HNF-4 alpha in the livers of IUGR rats was significantly lower than that in the normal rats $(P<.05)$ including newborn, 3week-old, and 8 -week-old rats. The results by real-time PCR were consistent with Western-blot analysis and immnohistochemistry staining. Conclusions. The decreased expression level of PDX-1 in the pancreas of IUGR rats is likely to result from the weakened regulation of HNF-1 alpha and HNF-4 alpha in the liver and contribute to the dysplasia of beta cells in pancreas, and it accordingly leads to diabetes mellitus. 


\section{Inborn Errors of Metabolism}

\section{Enzyme Replacement Therapy for \\ Mucopolysaccharidosis VI \\ (Maroteaux-Lamy Syndrome): \\ Experience in Hong Kong}

\author{
Wai Man But, ${ }^{1}$ Man Yee Wong, ${ }^{1}$ \\ Jasmine Chi Kwan Chow, ${ }^{1}$ Winnie Kwai Yu Chan, ${ }^{1}$ \\ Wai Tai Ko, ${ }^{1}$ Shun Ping Wu, ${ }^{1}$ May Ling Wong, ${ }^{1}$ \\ Ting Yat Miu, ${ }^{1}$ Wing Yee Tse, ${ }^{1}$ \\ Winnie Wai Yee Hung, ${ }^{2}$ and Tsz Wo Fan ${ }^{3}$ \\ ${ }^{1}$ Department of Paediatrics, Queen Elizabeth Hospital, \\ Hong Kong \\ ${ }^{2}$ Department of Physiotherapy, Queen Elizabeth Hospital, \\ Hong Kong \\ ${ }^{3}$ Department of Radiology \& Imaging, \\ Queen Elizabeth Hopsital, Hong Kong
}

Background. Mucopolysaccharidosis VI (MPS VI; Maroteaux-Lamy syndrome) is a very rare inherited lysosomal storage disease caused by deficient activity of the enzyme $\mathrm{N}$ acetylgalactosamine 4-sulfatase (arysulfatase B). Inevitably, the disease leads to significant functional impairment and shortened lifespan although intelligence is usually preserved. In the past, there is no satisfactory treatment although a few have benefited from bone marrow transplantation. Enzyme replacement therapy generates hope in the outlook for patients with MPS VI. Objective. We report our experience of the use of recombinant human arylsulfatase B (rhASB) as enzyme replacement therapy for MPS VI. Methods. A prospective study in which two patients with advanced MPS VI were enrolled to evaluate the efficacy and safety of weekly infusions of rhASB $1.0 \mathrm{mg} / \mathrm{kg}$. The primary efficacy variables were the distance walked in a 6-minute walk test (6MWT), forced vital capacity and ejection fraction. The secondary efficacy variables were the number of stairs climbed in a 3-minute stair climb (3MSC), joint mobility, the level of urinary glycosaminoglycan (GAG) excretion, autocontinuous positive airway pressure (CPAP) study, and liver size. Results. After 24 weeks of treatment, patient A walked 40 meters $(36 \%)$, and patient B walked 66 meters (58\%) more in the 6 MWT than in the baseline. Additional improvements were observed after receiving 48 weeks of rhASB. Patient A walked 142 meters (129\%) more in the 6 MWT and 33 stairs (60\%) more in the 3MSC than baseline while patient B walked 198 meters (174\%) more in the 6 MWT and 77 stairs (140\%) more in the 3MSC. Both showed significant decline in urinary GAG excretion after 12 weeks of treatment which remained low after 48 weeks. Improvements in joint range of motion were observed in both patients. The other efficacy variables remained static. There were no drug-related adverse events or allergic reactions reported during and after infusions of rhASB. Conclusions. rhASB significantly improves endurance and reduces urinary GAG excretion. There are improvements in range of joint movement after treatment. The drug is generally safe and well tolerated.

\section{Ring Chromosome 15 Syndrome: A Case Report and Literature Review}

\author{
Fang Xu, ${ }^{1,2}$ Chao Chun Zou, ${ }^{1}$ Li Liang, ${ }^{1}$ \\ and Xian Mei Huang ${ }^{2}$ \\ ${ }^{1}$ Department of Endocrinology, the Children's Hospital of \\ Zhejiang University School of Medicine \\ ${ }^{2}$ Department of Pediatrics, the First People Hospital of \\ Hangzhou
}

Objective. To report the clinical features, diagnosis, and management of ring chromosome 15 syndrome. Methods. There are case report and literatures review. Results. A 4.5year-old girl was admitted to our unit because of short stature. She was $86 \mathrm{~cm}$ in height and $9 \mathrm{~kg}$ in weight. Apart from short stature, sparse temporal hair, right simian crease, many irregular café-au-lait spots were observed on the chest, abdomen, and inner thigh (Figure 1). No mental retardation was found. Magnetic resonance imaging (MRI) scan of the brain as well as abdominal and cardiac ultrasonography were normal. Pelvic ultrasound showed that gonad was not found. Growth hormone $(\mathrm{GH})$ provocative tests showed normal GH peak. Cytogenetic analysis revealed a karyotype with 46, XX, $r$ (15) pattern. Recombination human GH (rhGH) with a dose of $0.1 \mathrm{U} / \mathrm{kg}-1 \cdot \mathrm{d}-1$ was administered for 4 months with height increment of $3 \mathrm{~cm}$, Figure 1 (not shown). Clinical features of the patient. (a) Short stature and sparse temporal hair. (b) Right simian crease. (c) and (d) Many irregular caféau-lait spots on the chest, abdomen, and inner thigh. (Please see poster for figures). Conclusion. These results showed that ring chromosome 15 syndrome should be considered in patients with short stature and café-au-lait spots. Timely recognition and hereditary tendency counseling are required. rhGH therapy improved growth and final height.

\section{Clinical, Biochemical, and Molecular Analysis of Combined Methylmalonic Acidemia and Hyperhomocysteinemia (cblC Type) in China}

\author{
Wang Fei, ${ }^{1}$ Lianshu Han, ${ }^{1}$ Yangyan Lin, ${ }^{2}$ \\ Yafen Zhang, ${ }^{1}$ Xiaolan Gao, ${ }^{1}$ and Yu Wang ${ }^{1}$ \\ ${ }^{1}$ Shanghai Children's Hospital Affiliated To \\ Jiaotong University (Worked) \\ ${ }^{2}$ Shanghai Institute For Pediatric Research, \\ Xinhua Hospital (Studied)
} Xuefan Gu, ${ }^{1}$ Jun Ye, ${ }^{1}$ Wenjuan Qiu, ${ }^{1}$ Huiwen Zhang, ${ }^{1}$

The most common inborn error of cobalamin metabolism in China is the cblC type, characterized by combined methylmalonic acidemia and hyperhomocysteinemia. The clinical presentation is relatively nonspecific such as feeding difficulty, recurrent vomiting, hypotonia, lethargy, seizures, progressive developmental delay and mental retardation, together with anemia, and metabolic acidosis; more specific 
biochemical findings include high levels of C3 (propionylcarnitine), C3/C0 (free carnitine), and C3/C2 (acetylcarnitine) measured by tandem mass spectrometry (MS/MS), elevation of MMA measured by gas-chromatography mass spectrometry (GC-MS), and increased total homocysteine with normal or decreased methionine. In this study, we report 50 Chinese patients with combined methylmalonic acidemia and hyperhomocysteinemia. Fourty six belonged to the cblC complementation group. Mutation analysis of the MMACHC gene was performed to characterize the mutational spectrum of cblC deficiency in China. A total of 17 different mutations were found in the MMACHC gene. Most of them clustered in exons 3 and 4, accounting for $91.3 \%$ of all mutant alleles. Two mutations were novel, namely c.315C > G (p.Y105X) and c.470G > C (p. W157S). In terms of genotype-phenotype correlation, the c.609G > A mutation was associated with early-onset disease when homozygous. Unlike previous reports from other populations, c.609G > A (p.W203X) was the most frequent cblC mutation detected in China, affecting 51 of 92 MMACHC alleles (55.4\%). The high prevalence of this nonsense mutation could have potential therapeutic significance for Chinese cblC patients. Besides traditional approaches consisting of hydroxocobalamin injections, carnitine, betaine, and protein restriction, novel drugs that target premature termination codons may have a role in the future.

\section{Inpatient Newborns Screening for Inherited Metabolic Disease}

\section{Liu Haihong, Yang Yao, and He xiyu}

\section{The Military General Hospital of Beijing PLA}

Objective. The feasibility of "second generation newborn screening" by tandem mass spectrometry was evaluated for the diagnosis of inherited metabolic diseases. The feasibility of newborn screening for inherited metabolic diseases in patients hospitalized is also investigated based on the experimental data. Methods. 4905 inpatient newborns, including 1723 premature newborns and 3182 full-term newborns, were selected from February 2009 to February 2010. The newborns were admitted to hospital as premature newborns, pneumonia, hyperbilirubinemia, hypoxic ischemic encephalopathy, diarrhoea, and so on. The tandem mass spectrometry was being used for amino acid and carnitine analysis by using dried blood-spot specimens routinely collected from newborns. Urine screening by the gas chromatography/mass spectrometry was conducted combined for the final diagnosed of inherited metabolic disease. Results. A total of 21 cases were diagnosis of inherited metabolic disease, including 8 full-term newborns and 13 premature newborns. The type of inherited metabolic disease and the corresponding number of cases are as follows: 5 cases of methylmalonic aciduria, 5 cases of hyperphenylalaninemia, 3 cases of short-chain fatty acid metabolism disorders, 2 cases of carnitine transport defect, 2 cases of isovaleric acidemia, 2 cases of tyrosinemia, and
2 cases of long-chain fatty acid metabolism disorder. Of the 21 inpatients, 13 cases with premature infant were hospitalized, and 4 cases with pneumonia were hospitalized, 4 cases with hyperbilirubinemia were hospitalized. The above data show that the incidence of inherited metabolic disease in inpatient newborns in our hospital was $4.28 \%$ ond is higher than the total incidence of inherited metabolic disease of $0.67 \%$. At the same time, the number of premature newborns for inherited metabolic disease is higher than that of patients with full-term newborns. Conclusion. The patients for diagnosis of inherited metabolic disease were hospitalized for common diseases. There were also no suspected clinical symptoms of inherited metabolic disease. It is suggested that the early diagnosis of asymptomatic newborns is important for the treatment. It is necessary to perform the newborn screening for inherited metabolic disease in inpatient newborns because of the high incidence of inherited metabolic disease in newborns hospitalized although the newborn screening was not developed nationwide.

\section{Six Years of Expanded Newborn Screening in China with Tandem Mass Spectrometry Results and Outcome}

\author{
Lian-shu Han, Jun Ye, Wen-juan Qiu, Hui-wen Zhang, \\ Xiao-lan Gao, Yu Wang, Wen-jun Ji, Jing Jin, \\ Xiao-yan Li, and Xue-fan Gu
}

\section{Department of Pediatric Endocrinology and} Genetic Metabolism, Xinhua Hospital, Shanghai Jiaotong University School of Medicine, Shanghai Institute for Pediatric Research, Shanghai

Objectives. The aims of this study were to determine the prevalence of amino acids disorders, organic acidemias, and fatty acid oxidation disorders in China by newborn screening using tandem mass spectrometry (MS/MS) and to assess the outcome of the patients that were diagnosed. Methods. From January 2003 to June 2010, 270067 neonates were collected in Shanghai. About 20 kinds of amino acids, free carnitine, and 40 kinds of acylcarnitines in their dry blood spots were tested by MS/MS for screening 40 kinds of amino acids disorders, organic acidemias, and fatty acid oxidation disorders. The positive neonates were confirmed with gas chromatography mass spectrometry (GC-MS) or gene mutation test. The mean period of clinical followup was 28.7 months per child ( 13 days $\sim 84$ months). Results. In total, 74 cases were diagnosed with 15 kinds of diseases. The overall prevalence was 1/3650. Among these cases, 41 cases (55.4\%) were diagnosed with 7 kinds of amino acids disorders (29 with hyperphenylalaninemia, 3 with maple syrup urine disease, 3 with hypermethioninemia $\mathrm{m}, 2$ with citrullinemia type II, 2 with tyrosinemia type II, 1 with homocystinuria, and 1 with Arginemia); 19 cases (25.7\%) were diagnosed with 4 kinds of organic acidemias (9 with 3-methyl crotonylCoA carboxylase deficiency, 8 with methylmalonic acidemia, 1 with propionic acidemia, and 1 with glutaric acidemia type I); 14 cases $(18.9 \%)$ were diagnosed with 3 kinds 
of fatty acid disorders (8 Carnitine uptake defect, 3 with short-chain acyl-CoA dehydrogenase deficiency, and 3 with medium-chain acyl-CoA dehydrogenase deficiency). During the follow-up period, all cases with 3-methyl crotonyl-CoA carboxylase deficiency, short-chain acyl-CoA dehydrogenase deficiency, and medium-chain acyl-CoA dehydrogenase deficiency, 6 cases with Carnitine uptake defect, and 4 cases with methylmalonic acidemia have no symptoms without treatment. Two cases with MSUD and propionic acidemia died at the age of a few days. Other cases have a good outcome with treatment. Conclusions. Newborn screening with MS/MS was valuable in the early diagnosis of severe and treatable for amino acid diseases, organic acidemias, and fatty acid oxidation disorders. These diseases were not rare in China. It was desirable that all newborns were screened with this method.

\section{Diagnosis of Tyrosinemia Type 1 by Determination of Succinylacetone in Dried Blood Spots using Tandem Mass Spectrometry and Treatment with Nitisinone}

\author{
Lian-shu Han, Yu Wang, Wen-jun Ji, Jun Ye, \\ Wen-juan Qiu, and Xue-fan Gu

\section{Department of Pediatric Endocrinology and Genetic Metabolism, Xinhua Hospital, Shanghai Jiaotong \\ University School of Medicine, Shanghai Institute for Pediatric Research, Shanghai}

Objective. To establish the method of determining the succinylacetone level in dried blood spots using tandem mass spectrometry for diagnosis of tyrosinemia type 1 and to evaluate the treatment effect of nitisinone for tyrosinemia type 1 . Methods. After the diameter of $3 \mathrm{~mm}$ dry blood spots was collected from 20 patients suspected with tyrosinemia and 1600 healthy children (as controls) were punched into wells of 96-well plate, $100 \mu \mathrm{L} 80 \%$ acetonitrile were added into each well, which contains hydrazine monohydrate and the internal standard C5-succinylacetone (SUAC). The eluates were transferred to another 96-well plate and dried under heated nitrogen, after the plate was incubated for 30 minutes at $65^{\circ} \mathrm{C}$. The residual hydrazine reagent was removed by the addition of $100 \mu \mathrm{L}$ methanol to each well and evaporation under heated nitrogen. The mobile phase (80\% acetonitrile) was added to each well, and $20 \mu \mathrm{L}$ samples were tested by tandem mass spectrometry for determining the level of SUAC. Two patients were treated with nitisinone $(1 \mathrm{mg} / \mathrm{Kg} / \mathrm{d})$ and feed with formula free of tyrosine and Phenylalanine. Results. The SUAC levels of nine patients were increased $(7.26-31.09 \mu \mathrm{mol} / \mathrm{L})$. The mean level of SUAC in 1600 controls was 2.68 (0.965.34) $\mu \mathrm{mol} / \mathrm{L}$. These patients were diagnosed as tyrosinemia type 1 with the symptoms of jaundice and hepatomegaly. Their tyrosine levels in blood were from 190 to $543 \mu \mathrm{mol} / \mathrm{L}$. Among them, two patients were treated with nitisinone and feed with formula free of tyrosine and Phenylalanine. After one month, their symptoms became better than before treatment. The SUAC levels decreased to normal (2.60,
$3.20 \mu \mathrm{mol} / \mathrm{L})$, but the tyrosine level increased. Conclusion. Determination of SUAC in dry blood spots using tandem mass spectrometry was a good method for the diagnosis of tyrosinemia type 1 . Nitisinone had a quick effect to decrease the SUAC level in blood of patients with tyrosinemia type 1 .

\section{Hyperthyroidism in a Girl with Down's Syndrome}

\section{Hui Huang and Yu Yang}

\section{Children's Hospital Of Jiangxi Province}

We describe the occurrence of hyperthyroidism and congenital heart disease in a 7-year-old girl with Down's syndrome. Thyroid disorders can occur in children with Down's syndrome while hypothyroidism occurs more frequently. There is no report about Down's syndrome associated with hyperthyroidism in China. The report of DS associated with hyperthyroidism and congenital heart disease is rare in other countries. We report this patient with thyroid disorders and Down's syndrome and emphasize the importance of periodic evaluation of thyroid function in children with Down's syndrome, instead of only detecting hypothyroidism.

\section{Clinical Characters of Mucopolysaccharidoses: 22-Case Report}

\section{Yonglan Huang, Sheyong Li, Xiaoyuan Zhao, and Li Liu}

\section{Department of Endocrinology and Metabolism, Guangzhou Women and Children's Medical Center, Guangzhou}

Objective. The objective of this study is to observe the differentiation of the clinical characteristics of various subtypes of mucopolysaccharidosis (MPS). Methods. From June 2008 to May 2010, twenty-two patients in our center were highly suspected to be suffering from MPS. Their ages range from 0.4 to 8.4 years old and averaged 3.0 years old. All the X-ray inspections of their bones exhibited MPSlike pathological changes. In all cases, urinary glycosaminoglycans (GAGs) content was detected by dimethylmethylene blue-tris spectrophotometry, and agar electrophoresis was applied for qualitative analysis. Fluorescence enzyme assays in leukocytes of peripheral blood for $\alpha$-L-iduronidase (MPS I), iduronate sulfatase (MPS II), arylsulfatse B (MPS $\mathrm{VI}$ ), $\beta$-D-glucuronidase (MPS VII), and $\beta$-D-galactosidase (MPS IVB) were done in our center for 20 cases. The other two patients were diagnosed as MPS IVA with enzyme assay in Beijing. Results. GAG levels as well as the ratios of GAG/creatinine in urine were obviously elevated in 19 cases, with urine GAG levels ranging from 127.7 to $775.6 \mathrm{mg} / \mathrm{L}$ and averaging $268.7 \pm 152.4 \mathrm{mg} / \mathrm{L}$. Dermatan sulfate and/or chondroitin sulfate were detected by electrophoresis in these 19 patients. Three patients had normal urinary GAG, and 2 of them were diagnosed as MPS IVA with enzyme assay in Beijing. Enzyme assay results of 20 patients in our center 
showed 4 cases with type I, 6 with type II, 3 with type VI, and one with type VII. Six cases cannot be classified. All classified cases had typical clinical features of MPS and high urinary GAG excretion. The 6 unclassified patients exhibited similar clinical features, characterized primarily by growth retardation, skeletal dysplasia, and normal intelligence development, which are similar to the clinical features of the cases diagnosed as type IVA in Beijing. Conclusion. Urinary GAG quantitative and qualitative analyses are still effective screening procedures for mucopolysaccharidosis except for type IV patients. Total urine GAGs in patients with MPS IVA are close to the normal range, so it is difficult to distinguish this type of MPS based on urine GAG excretion. Type II is still the most common type of MPS in this study.

\section{Enzyme Replacement Therapy in Patients Who Have Mucopolysaccharidosis and Are Younger than 5 Years Old}

\section{Se Hwa Kim, Sung Won Park, Young Bae Sohn, Sung Yoon Cho, Seon Tae Jee, and Dong-Kyu Jin \\ Department of Pediatrics, Samsung Medical Center, Sungkyunkwan University School of Medicine, Seoul, Korea}

Enzyme replacement of therapy (ERT) is one of the most promising therapeutic strategies for the treatment of lysosomal storage disorders. ERT is available in three types of Mucopolysaccharidosis (MPS): MPS I (Aludrazyme), MPS II (Elaprase), and MPS VI (Naglazyme) for patients who are over 5-year-old. But recently, early diagnosis can be done by expert clinicians and even in prenatal case. We describe the case of ERT under 5 years old MPS patients. Up to June, 2010 in Samsung Medical Center, there were 6 patients who were diagnosed as MPS and started ERT under 5 years old. 3 patients were MPS I, and other 3 patients were MPS II. 2 patients who was diagnosed as MPS I were female, and others were male. Their ages at diagnosis were 4 to 37 months old $(4,13,16,25,27$, 37 months old), and they are now 9 to 60 months old (9, 39, 32, 81, 60 months old). The youngest patient was started ERT at 4 month-old and others were started at their 13 to 49 months old $(13,29,27,28,49$ months old). First manifested symptoms of patients were macrocephaly, kyphosis, and coarse face appearance. Especially, in 2 of them, one was MPS I and the other was MPS II who had an elder brother with same disease. And the youngest one was diagnosed by the iduronate-2-sulfatase (IDS) gene analysis from chorionic villi sampling. His mother knew that she was a heterozygous carrier of IDS gene mutation because her younger brother died from MPS II. All of them were confirmed as MPS by the enzyme assay in leukocytes and fibroblast skin culture. We started ERT with $\alpha$-Liduronidase (Aldurazyme) to MPS I and did recombinant human iduronate-2-sulfatase (Elaprase) to MPS II patients as recommended dose for those over 5 years old. But for
MPS II patient who was 4 months old, we started ERT by recombinant human IDS (Elaprase) with reduced dose of $0.1 \mathrm{mg} / \mathrm{kg}$ and increased dose every 2 weeks by $0.1 \mathrm{mg} / \mathrm{kg}$ up to $0.5 \mathrm{mg} / \mathrm{kg}$ IV infusion. During ERT, all patients had no adverse effects, and the excretion of GAGs was decreased. We have evaluated other clinical symptoms such as liver/spleen volume, heart function, and neurologic evaluation. We describe a successful ERT to MPS I and MPS II patients under 5 years old without any adverse events. It indicates that ERT in young children is well tolerated and that it has several effects which may confer clinical benefits with longterm therapy.

\section{The Genotype and Phenotype of Phenylalanine Hydroxylase in Patients with Phenylketonuria}

\author{
Song Li, ${ }^{1}$ Dang Li-heng, ${ }^{1}$ Meng Ying-tao, ${ }^{1}$ \\ and Fu Bo-jing ${ }^{2}$
}

\section{${ }^{1}$ Pediatric Research Institute, Tianjin Children's Hospital, 300074, China \\ ${ }^{2}$ The Center Of Women And Children's Health In Tianjin 300070, China.}

Objective. To investigate the correlation of genotype and phenotype of phenylalanine hydroxylase (PAH) in patients with phenylketonuria, expecting it to be helpful for management and genetic counseling. Methods. All of 13 exons and flanking introns of PAH gene from 101 patients with high blood phenylalanine lever $(>120 \mu \mathrm{mol} / \mathrm{L})$ at initial diagnosis were amplified by polymerase chain reaction and analyzed by single-strand conformation polymorphism (SSCP), denaturing high-performance liquid chromatography (DHPLC) and DNA sequencing. The correlation between genotype and biochemical phenotype was observed by comparison. Results. A total of nine homoallelic mutant PAH genotypes were found (9\%) including $3 \mathrm{R} 111 \mathrm{X} / \mathrm{R} 111 \mathrm{X}, 1$ IVS4nt1g>a/IVS4nt-1g>a, 3 R243Q/R243Q, and 2 V399V/V399V genotypes. Eight of them (8/9) belong to classic PKU phenotypes except $1 \mathrm{R} 243 \mathrm{Q} / \mathrm{R} 243 \mathrm{Q}$ genotype, which fits moderate PKU phenotype. Among ninety-one heteroallelic mutant PAH genotypes (90.1\%), 53 kinds of mutations corresponded to 61 classic PKU phenotypes; 29 moderate PKU phenotypes and 1 mild PKU phenotype besides nonmutation were found in one case. Heteroallelic mutant PAH genotypes R111X/R243Q and Y204C/R243Q were observed in both classic PKU and moderate PKU phenotypes. Conclusions. In the present, ninety percent of genotypes were compound heterozygous. The independent assortment of mutation alleles results in the complicating relationship between genotype and phenotype. One should be cautious when evaluating the prognosis based on genotype during genetic counseling. The phenomenon which is the same genotype but with the different biochemical phenotype hints that some other influential factors might not be found during phenylalanine metabolic process. 


\section{Recurrent Severe Ketoacidosis Caused by SCOT Deficiency: A Case Report}

\author{
Li Liu, Xiaohong Zhang, Xiuzhen Li, and Jing Cheng \\ Department Of Endocine And Metabolism, \\ Guangzhou Women And Children's Medical Center
}

A 3-years-old boy had recurrent ketoacidosis six times triggered by only mild fever since 8 months old age. Each time he falls into deep breath, disturbance of consciousness, blood PH 6.9-7.1, BE-15-25 mmol/1, HCO3-3$7 \mathrm{mmol} / \mathrm{l}$, beta-hydroxybutyrate $3-5 \mathrm{mmo} / \mathrm{l}$, and lactic acid 10-12 mmol/l, urine organic acid analysis only sees a lot of ketones. Triglyceride, glucose, ammonia, bilirubin, liver, and muscle enzymes are all normal, with no visceral enlargement and fatty. No positive finding was found in cranial MRI. And he could recovery from his severe situation within 1 to 2 days with timely enough sodium bicarbonate correct acid, expansion, and glucose infusion. And there was no psychomotor retardation. His SCOT Gene was analyzed and found to be skipping of exon 12. Detection in DNA level showed a single-base substitution $(\mathrm{c} 1147 \mathrm{G}>\mathrm{A})$ in exon 12. The pre-mRNA secondary structure analysis revealed a change of pre-mRNA secondary structure after substitution while the ESE analysis showed that the purine-rich region was unlikely acting as ESEs, which was not reported before, and at the same time the 50 normal controls had no such mutation. We concluded that SCOT gene c1147G > A might cause skipping of exon 12, SCOT deficiency, and recurrent severe ketoscidosis.

\section{Sitosterolemia in Infants and Children}

\author{
Dau-Ming Niu,,2 Kah-Wai Chong, ${ }^{1}$ Ju-Hui Hsu, ${ }^{2}$ \\ Tina Jui-Ting Wu, ${ }^{2}$ Hsiao-Chi Yu, ${ }^{2}$ \\ Cheng-Hung Huang, ${ }^{2}$ Ming-Yu Lo, ${ }^{2}$ Ching Fai Kwok, ${ }^{3}$ \\ Lisa E. Kratz, ${ }^{5}$ and Low-Tone $\mathrm{Ho}^{4}$ \\ ${ }^{1}$ Institute of Clinical Medicine, School of Medicine, \\ National Yang-Ming University \\ ${ }^{2}$ Departments of Pediatrics \\ ${ }^{3}$ Medicine, and \\ ${ }^{4}$ Medical Research and Education, Taipei Veterans General \\ Hospital, Taipei, Taiwan, R.O.C. \\ ${ }^{5}$ Kennedy Krieger Institute, Baltimore, USA.
}

The clinical observation and treatment of young children with sitosterolemia have rarely been reported. We report clinical, biochemical, and molecular genetic observations and treatment outcomes for 5 Chinese children presenting with sitosterolemia from 4 separate families in whom we identified 2 new (Y329X, G269R) and 3 known (R446X, $\mathrm{N} 437 \mathrm{~K}, \mathrm{R} 389 \mathrm{H})$ mutations in the ABCG5 gene. The R389H mutation was found in $50 \%$ of alleles. Three of these five patients received cholestyramine therapy with a very good response. However, all of the patients discontinued this therapy because of poor compliance. Finally, all of these five patients were on ezetimibe therapy and had satisfactory total serum cholesterol levels though their plant sterols levels were still higher than normal. Another noteworthy finding is that a female infant had a serum cholesterol level of $654 \mathrm{mg} / \mathrm{dl}$ at 7 months of age, despite being breast fed (with very tiny amounts of plant sterols) since birth and undergoing 4 months of ezetimibe administration. Although she failed to respond to ezetimibe during this period, she did show improvement when the therapy was started again at 2 years of age. It is possible that another 23-month-old female patient also responded more slowly to ezetimibe treatment than older patients.

\section{Studies on Clinical Manifestation and SLC25A13 Gene Mutation of Chinese Patients with Neonatal Intrahepatic Cholestasis Caused by Citrin Deficiency}

\author{
Wenjuan Qiu, Yazhi Xing, Je Ye, Lianshu Han, \\ Huiwen Zhang, and Xuefan Gu \\ Department of Pediatric Endocrinology and Metabolic \\ disease, Xinhua Hospital Affiliated to Shanghai Jiaotong \\ University, Shanghai Institute for Pediatric Research
}

Objectives. To investigate the clinical and laboratory features of neonatal intrahepatic cholestasis caused by citrin deficiency (NICCD) and to characterize the molecular basis of this disease and prognosis. Methods. Thirty-one patients were detected because of idiopathic intrahepatic cholestasis and jaundice. Eighteen exons of SLC25A13 gene were analyzed. Mutations are verified by bidirectional sequencing of the whole detected exons. The clinical features, blood amino acid and acylcarnitine profiles, urinary organic profiles, and other laboratory data were reviewed and analyzed. Results. The NICCD patients showed low birth weight, and the average time of onset including jaundice and hepatomygly was 25 days. Laboratory data showed liver dysfunction, hyperbilirubinemia, hypoproteinemia, high levels of $\alpha$ fetoprotein, prolonged prothrombin time, hypoglycemia, and hyperammoniemia. MS-MS analysis of the blood sample revealed specifical elevation of citrulline and other amino acids. GC-MS analysis of the urine sample showed elevated 4-hydroxyl phenylpyruvic acid and 4-hydroxyl phenyllactic acid. 15 different mutations of SLC25A13 gene were identified, including 7 new mutations: G386V, K453R, D350N, R467X, 1192-1193delT, 1092-1095delT, and IVS11+5G > C. 51 mutated alleles were identified in the 56 alleles $(91.1 \%)$. 851del4, 1638ins23, and IVS6+5G > A mutations were the most frequent mutations in Chinese people, accounting for $39.2 \%, 17.7 \%$, and $14.7 \%$ of the total alleles examined, respectively. 4 in the 31 patients have poor prognosis, 3 died, and 1 implemented liver transplantation. No obvious relationship was found between the genotype and phenotype in NICCD. Conclusions. 15 mutations were found in 31 Chinese patients from 28 families, including 7 new mutations. 851del4, 1638ins23, and IVS6+5G > A mutations are frequent mutations in Chinese NICCD. There is no obvious relationship between the genotype and phenotype of NICCD. Not all of NICCD patients have optimistic 
prognosis, and the poor prognosis may be associated with delayed treatment.

\section{Gene Mutation Analysis in Fifteen Chinese Children with Wilsons's Disease}

\author{
Huiying Sheng, Li Liu, Xiuzhen Li, Jing Cheng, \\ and Youlan Huang
}

\section{Department of Endocrine and Metabolism, Guangzhou Women and Children's Medical Center}

Objective. To confirm the diagnosis of Wilsons's disease (WD) on the gene level and explore the mutation in Chinese Children by PCR-based restriction mapping analysis. Methods. Exon 8, exon 12, and exon 13 of ATP7B gene were amplified by PCR from genomic DNA, digested with restriction enzymes Msp I, Tai I, and BstDs I, respectively and analyzed by $1.8 \%$ gel electrophoresis of the cleavage products. The exons of gene mutation were confirmed by direct sequencing. Results. Arg778Leu of exon 8 was detected in $26.7 \%(4 / 15)$ patients. 1 patient was homozygous, and 3 patients were heterozygous for this mutation. Thr935Met of exon 12 was detected in 20\% (3/15) patients, and 2 cases were heterozygous for this mutation. None of patients had Pro992Leu of exon13. Among them, 1 case was compound heterozygous mutation with Arg778Leu/Thr935Met. The result of this method was the same as that of the DNA sequencing. Three family trees were built and analyzed by detecting these gene mutations. Conclusion. The method of restriction mapping can identify high-frequency mutational sites of WD gene accurately and rapidly and help to diagnose suspicious WD cases and their family members. The mutations of Arg778Leu and Thr935Met are "hot-spot" mutations in Chinese WD patients, whereas the Pro992Met would be confirmed by more samples.

\section{Cytogenetic and Molecular Genetic Analysis of Down's Syndrome: 287 Cases}

\section{Cui Song, Feng Xiong, and Min Zhu}

Special care ward, Children's Hospital, Chongqing Medical University

Objective. To better understand the karyotype distribution of the patients with Down's syndrome (DS) who have been finally diagnosed in our hospital. To compare the strengths of G-banding karyotype analysis and FISH (Fluorescence In Situ Hybridization, FISH) in diagnosing the DS patients with the chromosome mosaicism and calculating the percentage of abnormal karyotypes. Methods. Blood of the patients who were likely to suffer from DS was selected for G-banding karyotype analysis to describe the karyotype distribution. 5 of the finally diagnosed patients with the chromosome mosaicism were randomly selected for FISH to compare the percentage of abnormal karyotypes of the two methods. Results. 287 DS patients were diagnosed, 91.29\% of whom had the simple karyotype. Among the patients with the translocation karyotype, $11 \mathrm{D} / \mathrm{G}$ translocation karyotypes were detected, accounting for $73.33 \%$, while 4 G/G translocation karyotypes were diagnosed, constituting $26.67 \%$. One patient with both the translocation karyotype and the mosaicism karyotype was detected in our research, which was uncommon in recent report. The results of FISH and G-banding karyotype analysis were consistent. There was no significant difference of the abnormal karyotype rates for DS patients with the chromosome mosaicism by G-banding karyotype analysis and FISH. Conclusions. The simple karyotype is the main karyotype of the DS patients in our research. Among the translocation karyotype, the D/G kind is common. DS patients can be diagnosed efficiently by G-banding karyotype analysis, but we still suggest using FISH to get the proportion of the abnormal cell for the patients who have less triploid cells and slighter clinical manifestation.

\section{Spectrum of Inborn Errors of Metabolism in Vietnamese Patients: Clinical Presentation and Results of Treatment}

\author{
Nguyen Thi Hoan, ${ }^{1}$ Vu Chi Dung, ${ }^{1}$ \\ Nguyen Ngoc Khanh, ${ }^{1}$ Bui Phuong Thao, ${ }^{1}$ \\ Can Thi Bich Ngoc, ${ }^{1}$ Khu Th Khanh Dung, ${ }^{1}$ \\ Nguyen Thu Nhan, ${ }^{1}$ Seiji Yamaguchi, ${ }^{2}$ \\ and Nguyen Thanh Liem ${ }^{1}$

\section{${ }^{1}$ National Hospital of Pediatrics, Hanoi, Vietnam \\ ${ }^{2}$ Department of Pediatrics, Shimane University School of Medicine, Japan}

As hospitalizations for traditional pediatric illnesses have declined during the last century, due primarily to improved treatment of infectious diseases, the contribution of other disorders has gained prominence. Biochemical genetics, with its various inherited metabolic disorders (inborn errors of metabolism: IEM), has become more important in the routine care of hospitalized pediatric patients. We aim to study spectrum of IEMs, clinical characteristics, prevalence of IEMs, and survival rate of children with IEMs at National Hospital of Pediatrics (NHP) in Hanoi, Vietnam. 650 patients with suspected IEMs were examined and treated at NHP from 2005 to May, 2010. Quantitative analysis of blood amino acids and acylcarnitine using Tandem MS and urine organic acids using GC/MS was done in Shimane University, Japan. IEMs were identified in 81 cases (12.5\%) of suspected patients. IEMs occupied $0.033 \%$ of inpatients at NHP. Age ranged from 2 days to 18 years. Among 81 patients with IEMs, 54/81 cases (66.7\%) were organic acidemia, $17 / 81$ cases $(21 \%)$ were aminoacidopathy, and 10/81 cases $(12.3 \%)$ were fatty acid oxidation disorders. In organic academia, lethargy and metabolic acidosis were common features $(88.9 \%)$. Mental retardation and encephalopathy were frequent symptoms in cases with aminoacidopathy $(41.2 \%$ and $58.2 \%$, resp.) and in cases with fatty acid oxidation (30\% and $60 \%$, resp.). Nearly, the half of the cases had familial history with one or more siblings with similar presentation. Treatment saved lives without mental 
and motor development delay in $23.5 \%$ cases. Among 81 cases, $23.5 \%$ had mental and motor development delay. 17 patients of 54 cases with organic academia were without complication. In aminoacidopathy, 9/12 cases with MSUD died; 5 cases with PKU had mental delay. This study shows that organic acidemia is the most common of IEMs at NHP.

\section{Associations of Methylation Status in the MTHFR Promoterregion with Congenital Heart Diseases}

\section{Zhang Wendi and Luo Xiaoping}

Department of Pediatrics, Tongji Hospital, Tongji Medical College, Huazhong University of Science and Technology

Objective. The etiogenic mechanisms of congenital heart diseases (CHDs) are still poorly known. Singlenucleotide polymorphisms (SNPs) in the Methylenetetrahydrofolate reductase (Methylene tetrahydrofolate reductase, MTHFR) gene have been revealed to be involved in CHDs; however, other mechanisms of pathogenesis, for example, promoter region hypermethylation, may also play an important role. Therefore, in our study, we compared the methylation status of the promoter region of MTHFR in children with CHDs with children without congenital birth defects. We did it in order to get the message of the relation of the methylation status of the promoter region of MTHFR and CHDs. Methods. We collected peripheral venous blood samples of 53 patients with CHDs and 80 healthy children without congenital birth defects (controls) and abstracted DNA from leukocytes. Then, the DNA was modified by sodium bisulfite and analyzed by Methylation Specific PCR (MSP) amplification, which use primers that hybridize to the CpG islands in the promoter region of MTHFR. And the relation between the methylation status of the promoter region of MTHFR and CHDs was analyzed by case-control study. Results. In peripheral venous blood, the analysis result of MSP revealed that the complete methylation ratio in case $(2 / 53,3.77 \%)$ is slightly higher than control $(2 / 80,2.50 \%)$; the incomplete methylation ratio in case $(13 / 53,24.53 \%)$ is also slightly higher than control $(9 / 80,11.25 \%)$. But there is no statistical significance between them $(P=.110)$. Conclusions. There are no differences between children with CHDs and children without congenital birth defects about the methylation status of the promoter region of MTHFR. The causative mechanisms of CHDs are not due to the hypermethylation status of the promoter region of MTHFR.

\section{Effects of the Mitochondrial Respiratory Chain Complexii in Rat Cardiac Muscle by Methylmalonic Acid}

\author{
Qin Yi, Fengyan Tian, Hong Wei, Yan Liang, \\ Qin Ning, and Xiaoping Luo \\ Department of Pediatrics, Tongji Hospital, \\ Tongji Medical College, Huazhong University of Science \\ and Technology, Wuhan 430030, China
}

Objective. To clarify the underlying mechanisms involved in the mitochondrial respiratory chain by methylmalonic acid using mitochondria from cardiac muscle of young rats. Methods. Mitochondria were extracted from cardiac muscle of 30-days-old SD rats and incubated by different concentrations of methylmalonic acid $(0 \mathrm{mM}, 1 \mathrm{mM}, 2.5 \mathrm{mM}$, and $5 \mathrm{mM}$ ) and different times ( 30 minutes, $2 \mathrm{~h}, 4 \mathrm{~h}$ and $8 \mathrm{~h}$ ). The mitochondrial respiratory chain complexII enzymatic activity and expression of flavoprotein subunit 70 (FP) and iron-sulfur protein subunit 30 (IP) in complexII were, respectively, detected using spectrophotometric method and Western blot. Results. (1). Enzymatic activity of mitochondrial respiratory chain complexII. (1) When exposed to methylmalonic acid for 30 minutes and $2 \mathrm{~h}$, respectively, complexII activity in different concentrations groups had no significant difference with that in control group. (2) When exposed to methylmalonic acid for $4 \mathrm{~h}$, complexII activity in three treatment groups decreased significantly compared with the control group. (3) When exposed to methylmalonic acid for $8 \mathrm{~h}$, complexII activity in $1 \mathrm{mM}$ group had no significant difference with that in control group; but in $2.5 \mathrm{mM}$ and $5 \mathrm{mM}$ groups, complexII activity decreased significantly; (4) complexII activity remained stable when exposed to 30 minutes, $2 \mathrm{~h}$, and $4 \mathrm{~h}$; when the incubation time prolonged to $8 \mathrm{~h}$, complexII activity decreased significantly. (2). Expression of FP and IP in Western blot (1) when exposed to methylmalonic acid for 30 minutes, expression of FP and IP in treatment groups had no significant difference with those in control group; (2) when exposed for $2 \mathrm{~h}$, expression of IP in treatment groups had no significant difference with those in control group, but expression of FP decreased significantly; (3) when exposed for $4 \mathrm{~h}$, expression of IP in treatment groups had no significant difference with those in control group, but expression of FP decreased significantly; (4) when the incubation time prolonged to $8 \mathrm{~h}$, expression of FP decreased significantly in treatment groups; expression of IP in $1 \mathrm{mM}$ group had no significant difference with that in control group, but in $2.5 \mathrm{mM}$ and $5 \mathrm{mM}$ groups, it decreased significantly. Conclusions. Timedependent and concentration-dependent inhibition of respiratory chain complexII enzymatic activities and expression of protein subunits by methylmalonic acid found in rat heart mitochondria may suggest a direct inhibition mechanism of methymalonic acid on the respiratory chain complexII.

\section{A Case Report of Niemann-Pick Type C Disease}

\author{
Yining Zhang, ${ }^{1}$ Hongwei Du, ${ }^{1}$ Shanyu Li, ${ }^{1}$ \\ Lijie Wang, ${ }^{2}$ and Li Shibo ${ }^{2}$ \\ ${ }^{1}$ Endocrinology and Metabolism Department, Pediatrics, \\ First Hospital of Jilin University Changchun \\ ${ }^{2}$ The Department of Pediatrics, The university of \\ Oklahoma Health Science Center
}

A 12-year-old Chinese boy born to consanguineous parents presented with progressive neurological regression and hepatomegaly noticed after 8 year of age. Bone marrow examination revealed presence of storage cells suggestive of 
Gauchers or Niemann Pick. Confirmatory study by lysosomal enzyme from leucocytes was normal for $\beta$-Glucosidase and sphingomyelinase specific for Gauchers and Niemann Pick type A or B, respectively. Further study was carried out on Genetic testing for the boy and his parents. The mutations in NPC1 were detected in the boy confirming the diagnosis of Neimann-Pick type C (NPC), a rare autosomal recessive disorder. Meanwhile, his parents were confirmed as NPC carriers. The mutations found were Mother: g 46639C $>C / T$; p 927A > A/V; Father: g 46636C>C/T; p 936A >A/V; patient: $g$ 46639C $>\mathrm{C} / \mathrm{T} ; 46636 \mathrm{C}>\mathrm{C} / \mathrm{T} ; \mathrm{p} 927 \mathrm{~A}>\mathrm{A} / \mathrm{V}, 936 \mathrm{~A}>\mathrm{A} / \mathrm{V}$.

\section{Thyroid Disorders}

\section{Children Beyond 5 Years of Age: Are We Still Diagnosing Congenital Hypothyroidism So Late?}

\section{Anju Seth, Varun Aggarwal, and Anu Maheshwari}

\section{Department of Pediatrics, Kalawati Saran Children's \\ Hospital and Lady Hardinge Medical College, New Delhi-110001, India}

Background. India lacks a neonatal screening program for detection of congenital hypothyroidism $(\mathrm{CH})$ leading to a delay in its diagnosis. Tardiness in diagnosis and treatment of $\mathrm{CH}$ leads to significant neurological morbidity. Objective. To determine the frequency of undiagnosed and untreated congenital hypothyroidism in children presenting after 5 years of age. Methods. Retrospective analysis of records of children with hypothyroidism who were more than 5 years of age at presentation to Pediatric Endocrinology clinic at Kalawati Saran Children's hospital, New Delhi, India, between October 1997 and April 2010. Results. During the study period, 320 children were diagnosed with hypothyroidism. Among these, 94 (29.3\%) were older than 5 years of age at presentation. In this group of subjects, $\mathrm{CH}$ was diagnosed in $34(36.1 \%)$ and acquired hypothyroidism (AH) in $60(63.9 \%)$. Female preponderance was more commonly seen in $\mathrm{AH}(83.3 \%)$ as compared to $\mathrm{CH}(61.7 \%)$. Symptoms of $\mathrm{CH}$ were noted at a mean age of $35.3 \pm 25.9$ months (range 12-132 months). However, the children were brought for evaluation at our center at the age of $86.6 \pm 35.3$ months (range 60-216 months). The mean gap between onset of symptoms and diagnosis was nearly 51 months. However, the mean duration of symptoms before diagnosis in children with $\mathrm{AH}$ was significantly shorter (8 months, $P<.001)$. The main presenting complaints in children with $\mathrm{CH}$ and $\mathrm{AH}$ were growth delay (100\%) and thyroid swelling $(65 \%)$, respectively. Anterior fontanel was still open in 10 children with $\mathrm{CH}$ while one child also had open posterior fontanel. Mean fT4 values of $\mathrm{CH}$ and $\mathrm{AH}$ at presentation were 0.23 and $0.34 \mathrm{ng} / \mathrm{dL}$, respectively $(P=.19)$. Children with $\mathrm{CH}$ had significantly higher TSH values $(377.4 \mathrm{mIU} / \mathrm{L})$ as compared to those with $\mathrm{AH}(151.4 \mathrm{mIU} / \mathrm{L})(P=.002)$ and had significantly delayed bone age (mean chronological age minus bone age 4.9 years in $\mathrm{CH}$ versus 2.0 years in $\mathrm{AH}$,
$P<.01)$. The most common cause of congenital hypothyroidism was thyroid dysgenesis $(51.8 \%)$, followed by agenesis $(25.9 \%)$ and dyshormonogenesis (22.2\%). Conclusions. In The absence of a universal screening program, diagnosis of $\mathrm{CH}$, an important cause of preventable mental retardation, is often delayed in India, even in the capital city. Until universal screening is implemented, a high awareness of this disorder among physicians is crucial to facilitate early diagnosis.

\section{Varied Clinical Presentations of Van Wyk Grumbach Syndrome}

\author{
Ahila Ayyavoo ${ }^{1}$ and Palany Raghupathy 1,2,3,4 \\ ${ }^{1}$ G. Kuppuswamy Naidu Memorial Hospital, \\ Coimbatore, India \\ ${ }^{2}$ Sagar Hospital, Indira Gandhi Institute of Child Health \\ ${ }^{3}$ Kempegowda Institute of Medical Sciences \\ ${ }^{4}$ Bangalore, India
}

Sexual precocity, hypothyroidism, and large cystic ovaries in young girls, though unusual, were seen in eight girls over a 5-year period. This paper describes varying clinical manifestations in eight girls presenting with van Wyk Grumbach syndrome (vWGS) and their response to thyroxine therapy. Clinical, biochemical and imaging features of eight children with vWGS treated during 2005-2009 and followup results of therapy are presented. Age at presentation ranged from 6 to 11 years. At first visit, clinical features of hypothyroidism were evident in $8 / 8$ girls, short stature (height: $<3 \mathrm{c}$ ) $6 / 8$, early thelarche $8 / 8$, sexual hair $0 / 8$, and precocious menarche (mean age at onset: 6.38 years) $7 / 8$. One girl aged 11 presented with thelarche, large abdominal masses and ascites. Two girls investigated with MRI brain scan for precocious puberty were referred as pituitary tumours; one of them (aged 6.5 years) had Down syndrome. Bone age was delayed in all though they had precocious puberty. Biochemical hypothyroidism was documented in all; TSH ranged from 118 to $4236 \mathrm{mIU} / \mathrm{ml}$. Ovarian hyperstimulation was demonstrated by ultrasonogram in all girls and very large ovarian cysts in two. One of them presented acutely with torsion of enlarged ovaries and required excision of a gangrenous ovary. Another girl was referred with a diagnosis of ovarian carcinoma. All girls improved with thyroxine therapy-loss of body weight upto $20 \%$; improved height velocity (mean: $9.5 \mathrm{~cm} /$ years), good catch-up growth, and crossover to higher centiles were noted in all, and signs of hypothyroidism abated well. Four girls attained puberty appropriately (mean: 12.6 years). All had regression of ovarian size. All were investigated earlier for precocious puberty, short stature or obesity and obvious hypothyroidism had been missed. Complications such as growth retardation in all, torsion of the ovary, the diagnosis of pituitary tumour (thyrotroph hyperplasia) in two girls and ovarian carcinoma in one may have been avoided by early recognition and treatment of vWGS. This condition may be easily treated simply with thyroxine replacement producing an assured outcome of excellent results, namely, arrest of puberty, improved physical growth and overall well-being. 


\section{Multicystic Ovaries in a Girl with Primary Hypothyroidism}

\author{
Sioksoan Chan-Cua \\ Department of Pediatrics, University of the Philippines, \\ Manila Doctors Hospital, Manila, Philippines
}

A girl presented with obesity and short stature, associated with recurrent abdominal pain, postprandial vomiting and constipation. She has gained much weight since age 4 years. Vaginal bleeding first occurred at the age of 8 years and her abdomen has increased significantly in size about a month prior to consultation. At the age of 11 years and 4 months, her height was $106.4 \mathrm{~cm}$ (height age of 4.8 years) and body mass index was $34(>+3 \mathrm{SD}$, WHO reference). Her blood pressure was $107 / 77 \mathrm{mmHg}$. She had dry skin, with pustules and abscess in the extremities, and acanthosis nigricans in neck, axilla, elbows, knuckles and knees. She had no palpable thyroid gland. Her breast development was at Tanner stage 4 and nipples were hyperpigmented. Her abdomen was globular and circumference was $91 \mathrm{~cm}$. Her bone age was 4.5 years at chronological age of 11 year 4 months. Thyroid ultrasonography showed small-sized thyroid gland, both lobes appeared small and displaced cephalad in the anterior neck at the midline. Abdominal Xrays showed segmental ileus. Initial pelvic ultrasonography showed bilaterally enlarged ovaries with multiple enlarged follicular cysts. Blood tests showed low free T4 level, $0.16 \mathrm{ng} / \mathrm{dl}$, high TSH level, $98.79 \mathrm{uIU} / \mathrm{ml}$, elevated prolactin level, $71.81 \mathrm{ng} / \mathrm{ml}$, FSH, $7.05 \mathrm{mIU} / \mathrm{ml}$, low LH, $0.10 \mathrm{mIU} / \mathrm{ml}$, estradiol, $171 \mathrm{pg} / \mathrm{dl}$, low IGF-1 level, $64.9 \mathrm{ng} / \mathrm{ml}$ and growth hormone $0.12 \mathrm{ng} / \mathrm{ml}$, dyslipidemia-high serum cholesterol, $10.06 \mathrm{mmol} / \mathrm{L}(390 \mathrm{mg} / \mathrm{dl}), \mathrm{LDL}, 7.64 \mathrm{mmol} / \mathrm{L}(294 \mathrm{mg} / \mathrm{dl})$ and triglycerides, $2.6 \mathrm{mmol} / \mathrm{L}(230 \mathrm{mg} / \mathrm{dl})$ and elevated liver enzymes (SGPT, $89 \mathrm{U} / \mathrm{L}$ and SGOT, $78 \mathrm{U} / \mathrm{L}$ ). Levothyroxine therapy was started at $200 \mathrm{mcg} / \mathrm{day}(5 \mathrm{mcg} / \mathrm{kg} / \mathrm{day})$ for the primary hypothyroidism. After 4.5 months, her BMI was reduced to 21.7 and her growth velocity increased to $11 \mathrm{~cm} /$ years. Restoration of euthyroid state was associated with decreased abdominal circumference and resolution of the ovarian cysts as well as normalization of biochemical parameters. This case emphasizes the need to consider hypothyroidism in the short girl with ovarian cysts. Right diagnosis with the initiation of levothyroxine therapy can reverse the symptoms and avoid unnecessary surgery on the ovaries.

\section{The Therapy of Low-Dose Levothyroxine to Central Nervous System Infection}

\section{Agung Tanurahardja, Antonius Pudjiadi, Aman Pulungan, Pramita Gayatri, and Risma Kaban}

\section{Indonesian Pediatric Society}

Objective. To study the therapeutical effect of low-dose levothyroxine $(2 \sim 3 \mu \mathrm{g} / \mathrm{kg} . \mathrm{d})$ in the infection of central nervous system (CNS) with hypofunction of thyroid. Method. Clinical data of 50 patients with CNS infection were analyzed
Free triiodothyronine (F13), triiodothyronine (T3), free thyroxin (FI'4), thyroxine (T4), and thyroid stimulating hormone (TSH) were determined, low-dose levothyroxine was used to treated such patients and the mind and cognition were test every day. They were divided into two groups randomly, one was given Levothyroxine, the other nothing. Results. Low FT3 was $66.7 \%$ in the abnormal thyroid function; it was obviously lower than the normal $(P<.05)$. The mind and cognition of patients with Levothyroxine became better than without treated. Conclusion. Levothyroxine can promote patients' mind and cognition to become better; it needs to be used early in CNS infection with hypofunction of thyroid.

\section{A 15-Year-Old Girl with Graves' Disease Associated with Moyamoya Disease}

\section{Chong Kun Cheon and Su Yung Kim}

Department of Pediatrics, Children's Hospital,

Pusan National University, Beomeo-ri, Mulgeum-eup, Yangsan-si, Gyeongnam, 626-770, Korea

Background. Thyroid hormone excess in Graves' disease is harmful to arterial walls because it may alter vascular reactivity, and Graves' disease is often accompanied by many cardiovascular symptoms. There have been few reported cases of the coexistence of Graves' disease and moyamoya disease. Clinical Case. A 15-year-old girl who had suffered from Graves' disease for seven years visited our hospital with central type right facial palsy. She presented with a clinical picture of hyperthyroidism, goiter and ischemic attack consisting of right-side hemiparesis. Thyroid function tests demonstrated suppressed thyroid-stimulating hormone and elevated thyroid hormone levels. The patient was diagnosed with Moyamoya disease with typical stenosis of the ICA and MCA, based on MRI scans and cerebral angiography. Single photon emission computed tomographic (SPECT) scans revealed cerebral hypoperfusion of the left frontal lobe, left thalamus without vascular reserve. The patient underwent cranial revascularization by encephalo-duro-arteriosynangiosis (EDAS). After surgery, the patient's symptoms improved, and the power of the right upper and lower extremities recovered. Conclusion. To our knowledge, this is the first case of Moyamoya disease associated with thyrotoxicosis in a young girl in Korea. Moyamoya disease should be considered in the differential diagnosis of cerebrovascular events in patients with Graves' disease.

\section{A Case of Melkersson-Rosenthal Syndrome in a Girl with Hashimoto's Thyroiditis}

\section{Chong Kun Cheon and Su Yung Kim}

\author{
Department of Pediatrics, Children's Hospital, \\ Pusan National University, Beomeo-ri, Mulgeum-eup, \\ Yangsan-si, Gyeongnam, 626-770, Korea
}

Background. Melkersson-Rosenthal syndrome (MRS) is a rare disorder of unknown etiology. MRS is classically defined 
as a triad of recurrent orofacial edema, relapsing paralysis of the facial nerve, and fissured tongue. It frequently occurs in the first two decades of life. The incidence of this syndrome is estimated to be about $0.08 \%$. A link between MRS and immunological disorders such as sarcoidosis, Crohn's disease, unilateral anterior uveitis and multiple sclerosis was documented. However, there have been few reported cases of the coexistence of Hashimoto's thyroiditis and MRS. We present here a case of MRS with the classical triad of symptoms and Hashimoto's thyroiditis. Clinical Case. A 10-year-old girl who had suffered from Hashimoto's thyroiditis for one year visited our hospital with peripheral type right-sided facial palsy. Antithyroperoxidase antibodies (anti-TPO) were highly elevated and thyroid function tests had evidenced a clinically significant hypothyroidism. The patient had a history of right-sided facial paralysis at the age of 9. The physical examination showed that the patient had a fissured tongue, right seventh nerve paralysis and mild orofacial edema. The examination of all other systems was normal. Nerve conduction study revealed paralysis of the right facial nerve. No pathological findings were detected on cranial MRI scan. After oral steroid therapy, the patient's symptoms improved. Conclusions. The presence of the antiTPO antibodies in the case reported here could suggest a possible correlation between immunological alteration characteristic of autoimmune thyroiditis and MRS. MRS should be considered in the differential diagnosis of relapsing paralysis of the facial nerve in patients with Hashimoto's thyroiditis. To our knowledge, this is the first case of MRS associated with Hashimoto's thyroiditis in a young girl.

\section{Neonatal Screening for Congenital Hypothyroidism: Twelve Years of Experience in Hat Yai Hospital}

\section{Pathikan Dissaneevate and Jeerawan Wannaro}

\section{Department of Pediatrics, Hat Yai Hospital, Songkhla, Thailand}

Objectives. To evaluate the incidence of congenital hypothyroidism $(\mathrm{CH})$ in neonates born at Hat Yai Hospital. Methods. TSH screening for $\mathrm{CH}$, using dried blood spot at the aged of 2 days or older, was introduced in Hat Yai Hospital since 1998. From January 1998 to March 2010, 77, 242 neonates born at Hat Yai Hospital were screened for $\mathrm{CH}$ at the aged of 2-3 days. Infants with elevated TSH level of greater than $25 \mathrm{mU} / \mathrm{L}$ were recalled for evaluated serum total T4, total T3 and TSH level. Thyroid scan and uptake were performed in affected infant after treatment at the aged of 2-3 years. Results. The coverage rate of TSH screening was $81 \%$ in 1998 and increased to $99.9 \%$ in 2010 . The average recall rate was 0.2 percent. Elevated TSH more than $25 \mathrm{mU} / \mathrm{L}$ was found in 172 neonates. Of these, 165 were repeated for TT4 or FT4, TT3 and TSH and only 21 were confirmed $\mathrm{CH}$. The incidence of $\mathrm{CH}$ was 1:3, 678 live-births. All affected infants were asymptomatic. Thyroid scan was performed in 15 infants which showed abnormal in 6 cases, ectopic thyroid in 5 neonates and thyroid dysgenesis in 1 neonate. Six infants were suggested as having thyroid dyshormonogenesis. When primary $\mathrm{CH}$ was confirmed, the treatment with thyroxine will be started. Conclusion. The incidence of $\mathrm{CH}$ at Hat Yai Hospital is 1:3, 678 live births. The cutoff value of serum TSH was $25 \mathrm{mU} / \mathrm{L}$. Routine neonatal screening for $\mathrm{CH}$ and early treatment should be established and continued to detect and prevent the intellectual handicap.

\section{Thyroid Dysfunction Detected in Intensively Cared Neonates for More Than 1 Month}

\section{Heon-Seok Han and Jae Hong Yu \\ Department of Pediatrics, Chungbuk National University Hospital, Joy children's Hospital}

Objective. To evaluate the incidence, time of detection, classification and the risk factors of thyroid dysfunction in very low birth weight (VLBW) and sick infants in order to help with the diagnosis and treatment of thyroid dysfunction in the neonatal intensive care unit (NICU). Methods. We reviewed medical records of 67 infants, who were admitted for more than 1 month in the NICU at Chungbuk National University Hospital from July 2006 through June 2009. At least two thyroid function tests (TFT) were performed in these infants, an initial screening within 2 weeks of age and a repeated TFT after 2 weeks of age. They were grouped as VLBW (birth weight $<1,500 \mathrm{~g}, n=40$ ) and non-very low birth weight (NVLBW, birth weight $\geq 1,500 \mathrm{~g}, n=$ 27). Results. At initial screening test, abnormal TFT was detected in 10 infants (9 VLBW, 1 NVLBW), including 1 primary hypothyroidism (PH), 2 hypothyroxinemia, and 7 hyperthyrotropinemia. Nine of them showed subsequent abnormal TFT, and 1 hypothyroxinemic VLBW changed to normal at followup. Additional 33 infants (22 VLBW, 11 NBLBW) showed abnormal subsequent test mostly at 2-6 weeks of age (90.9\%), resulting abnormal TFT in 42 $(62.7 \%)$ infants with $30(75.0 \%)$ in VLBW. The types of thyroid dysfunction (Table 4) were $\mathrm{PH}$ with marked TSH rise $(n=7)$, $\mathrm{PH}$ with delayed mild TSH rise $(n=14)$, hypothyroxinemia $(n=7)$, and euthyroid sick syndrome $(n=14)$. Among 42 infants with thyroid dysfunction 15 (35.7\%) were treated with thyroxine. Only the birth weight was significantly related to the risk of thyroid dysfunction $(P<.05)$. Conclusion. Thyroid dysfunction was very common in sick infants in NICU, especially in the VLBW. Frequently they were not detected at the initial screening, but detected at later repeated tests. The repeated thyroid function tests should be performed in NICU infants for later detection of thyroid dysfunction after 2-4 weeks of age. 
TABLE 4: Classification of thyroid dysfunction in subsequent followup test.

\begin{tabular}{lccc}
\hline Classification & VLBW & NVLBW & Total \\
\hline Thyroid dysfunction, no. $(\%)$ & $30(75.0 \%)$ & $12(44.4 \%)$ & $1\left(1^{*}\right)$ \\
PH with delayed marked TSH rise & $6\left(6^{*}\right)$ & & \\
PH with delayed mild TSH rise & & $1\left(1^{*}\right)$ & $7\left(7^{*}\right)$ \\
2(a) decreased FT4: mild PH & $2\left(1^{*}\right)$ & $4\left(1^{*}\right)$ & $3\left(2^{*}\right)$ \\
2(b) normal FT4: hyperthyrotropinemia & $7\left(3^{*}\right)$ & $2\left(1^{*}\right)$ & $4\left(4^{*}\right)$ \\
Hypothyroxinemia & $5\left(1^{*}\right)$ & $4\left(2^{*}\right)$ & 14 \\
Euthyroid sick syndrome & 10 & $4(33.3 \%)$ & $15(35.7 \%)$ \\
\hline Thyroid supplementation, no. $(\%)$ & $11(36.7 \%)$ & &
\end{tabular}

Values are represented as number and percent. ${ }^{*}$ Number of infants with thyroxine supplementation.

\section{Chronic Kidney Disease in a Child with Hashimoto Thyroiditis}

Zuhrotul Aini, ${ }^{1}$ Risky Vitria Prasetyo, ${ }^{1}$ Achmad Yuniari Heryana, ${ }^{2}$ Muhammad Faizi, ${ }^{2}$ Ninik Asmaningsih Soemiarso, ${ }^{1}$ Netty Harjantien, ${ }^{2}$ and Mohammad Sjaifullah Noer ${ }^{1}$

\author{
${ }^{1}$ Division of Nephrology \\ ${ }^{2}$ Division of Endocrinology, Department of Child Health, \\ Faculty of Medicine, University of Airlangga, \\ dr. Soetomo Hospital Surabaya
}

Background. Hashimoto thyroiditis is the most common cause of acquired hypothyroid. Severe hypothyroidism is associated with significant effects on the kidney due to low cardiac output, decrease in renal blood flow and glomerular filtration rate. Undiagnosed and untreated hypothyroid can cause chronic complication such as chronic kidney disease (CKD). Objective. To report a case of CKD in a child due to Hashimoto thyroiditis focusing on diagnosis and management. Case Report. A 7-year-old girl was referred with suspicion of hepatorenal syndrome. She suffered from vomiting for 1 month and easy fatigability, lack of appetite and constipation for 1 year. She had poor growth, and her height was not progressing since she was 4 years old. On physical examination we found short stature (weight at 25 th percentile, height at less than 3 rd percentile), dull expression, myxedema and dry skin. There was no goiter. Her blood pressure was $140 / 90 \mathrm{mmHg}$. Laboratory test revealed ALT $58 \mathrm{IU}$, AST $47 \mathrm{IU}, \mathrm{BUN} 21.0 \mathrm{mg} / \mathrm{dl}$, creatinine $2.2 \mathrm{mg} / \mathrm{dl}$, GFR $23 \mathrm{ml} / \mathrm{mn} / 1.73 \mathrm{~m}^{2}$, T4 $1.0 \mu \mathrm{g} / \mathrm{dl}(4.6-11)$, FT4 $0.4 \mathrm{ng} / \mathrm{dl}(0.8-2.0)$ and TSH more than $26 \mu \mathrm{IU} / \mathrm{ml}$ (0.4-7.0). Antithyroperoxidase antibody was $6315.92 \mathrm{IU} / \mathrm{ml}$ $(<56 \mathrm{IU} / \mathrm{ml})$ and antithyroglobulin positive was $1: 2.560$ ( $\mathrm{N}$ : negative). Abdominal USG revealed bilateral chronic parenchym kidney disease. Thyroid USG revealed shrunken thyroid lobes. The patients improved clinical and laboratory findings after treatment with levothyroxin and conservative treatment of CKD. Conclusion. Delayed diagnosis of hypothyroid can cause complications such as CKD that are usually overlooked and may result in potential diagnostic pitfalls. Thyroid hormone therapy led to significant improvement in thyroid and renal functions in our patient.

\section{Receiver Operating Characteristic Analysis of Cord Blood TSH for the Screening of Primary Congenital Hypothyroidism}

Suhaimi Hussain, Yazid Jalaludin, and Fatimah Harun

Pediatric Department, University Hospital, Jalan Hospital

Objective. To determine the safe cut off point for the screening of congenital hypothyroidism using ROC analysis. Methods. All newborns were screened for congenital hypothyroidism using cord blood TSH. TSH is analyzed by chemiluminescent method. Normally when the TSH is more than $20 \mathrm{mIU} / \mathrm{L}$, the repeated test for $\mathrm{TSH}$ and freeT4 will be done. When the test is abnormal the baby will be called for confirmation with venous blood for thyroid functions. ROC curves were calculated for cord blood TSH. Results. Six thousand eight hundred and sixty two $(n=6862)$ samples were analysed. The mean/median/standard deviation of TSH were 7.31/5.80/5.703. When the cutoff value of TSH used was 57 , the area under the curve was $0.9 \pm 0.130(\mathrm{SE})(95 \% \mathrm{CI}$ 0.644-1.156). With cut off value of TSH 20, the area under the curve was $0.553 \pm 0.041$, (95\% CI 0.472-0.633). It was noted that the lower the cutoff value for TSH, the area under the curve will be less accurate in discriminating cases and the control. Conclusion. Using cord blood TSH level of 20 is adequate and safe to screen congenital hypothyroidism in our population.

\section{Concurrent Occurrence of Chronic Lymphocytic Thyroiditis with Hypothyroidism and Growth Hormone Deficiency in a Turner's Syndrome Patient}

\author{
Wu Jin, Xiang ChengFa, Sun XiaoMei, and Yang Fan \\ Department of Pediatrics, Second Hospital of West China, \\ Sichuan University, Chengdu, China
}

Background. Concurrent deficiencies of three hormones in patients with Turner Syndrome have been rarely reported. Here we describe a case of a young girl who had Turner's syndrome with concomitant chronic lymphocytic thyroiditis, growth hormone deficiency, and hypothyroidism with cardiopericarditis. Case. An 11-year-old girl was referred to 
the outpatient clinic because of short stature, ochronosis and cardiopalmus. Her ultrasound revealed absence of ovarian tissue. Karyotype examination suggested Turner's syndrome with sex-hormone deficiency. She was found to have an abnormal thyroid gland and elevated thyroid-stimulating hormone (TSH). A positive thyroid autoantibody titer confirmed the diagnosis of chronic lymphocytic thyroiditis with hypothyroidism. Furthermore, her growth hormone levels were well below normal. Diagnosis. A multiendocrine disorder, that is, Turner's syndrome with chronic lymphocytic thyroiditis, growth hormone deficiency, and hypothyroidism with cardiopericarditis was diagnosed. Growth hormone and thyroxin substitution therapy was suggested.

\section{Primary Hypothyroidism Masquerading as Pituitary Tumor}

\section{Ansu Mali Joshi, ${ }^{1}$ VP Jyotsna, ${ }^{1}$ Emmy Grewal, ${ }^{1}$ Raju Sharma, ${ }^{2}$ and Ariachery C Ammini ${ }^{1}$}

${ }^{1}$ Departments of Endocrinology and
${ }^{2}$ Radiology AIIMS, New Delhi, India

Introduction. Hypothyroidism is a common endocrine disorder which usually presents with growth deceleration in children. Here we present a case of primary hypothyroidism presenting with a large pituitary mass at the age of 16. Case Report. Ms R.S., 16 years, was referred to us for management of pituitary macroadenoma. She gave history of headache off and on for the past 8 years and blurring of vision for 2 months. She also complained of increasing lethargy, cold intolerance, constipation and hoarseness of voice for the previous 6 years. She had attained menarche at 13 years of age. Physical examination revealed height of $140 \mathrm{~cm}$ (midparental height $154.5 \mathrm{~cm}$ ) and weight $35 \mathrm{~kg}$. Her pulse was $70 /$ minutes, BP was $120 / 70 \mathrm{~mm} \mathrm{Hg}$, skin was dry and rough. She was pale, and thyroid was not palpable. Visual acuity and visual field were normal. Deep tendon reflexes were delayed. Other systemic examination was essentially normal. Her hemoglobin was $9 \mathrm{gm} \%$. Total T4 was $0.64 \mu \mathrm{g} / \mathrm{dl}$ and TSH was $>100 \mu \mathrm{U} / \mathrm{ml}$. LH $4.3 \mathrm{mIU} / \mathrm{ml}$, FSH $5.5 \mathrm{mIU} / \mathrm{ml}$, ACTH $17 \mathrm{pg} / \mathrm{ml}$, prolactin $67.6 \mathrm{ng} / \mathrm{ml}$, cortisol $14.1 \mathrm{~g} / \mathrm{dl}$. ECG showed low voltage complexes. MRI sella showed enlarged pituitary gland measuring $2.99 \times 2.78 \mathrm{~cm} \times 1.89 \mathrm{~cm}$ with suprasellar extension with multiple cystic areas. She was started on thyroxin $25 \mathrm{~g} /$ day and gradually increased to $75 \mathrm{~g} /$ day. On followup at 4 months, total T4 was $11 \mathrm{~g} / \mathrm{dl}$ and TSH was $1.39 \mathrm{IU} / \mathrm{ml}$. Serum prolactin was within normal limits. MRI done at this time showed significant reduction in size. Discussion. Pituitary enlargement in patients with long-standing primary hypothyroidism is well known. The present case was unique for the fact that the mass had heterogeneous appearance with cystic areas making it difficult to differentiate from a pituitary tumor. The reduction in size following thyroxin substitution suggests that it was thyrotroph hyperplasia.

\section{Remission Predictors of Graves Disease in Children}

Shin Mi Kim, ${ }^{1}$ Sei Won Yang, ${ }^{1}$ Choong Ho Shin, ${ }^{1}$ Young Ah Lee, ${ }^{1}$ Min Jae Kang, ${ }^{1}$ and Jin Soon Hwang ${ }^{2}$

\author{
${ }^{1}$ Seoul National University College of Medicine \\ ${ }^{2}$ Ajou University School Medicine
}

Objectives. Antithyroid drug treatment is usually recommended as the initial treatment for children with Graves' disease. However, achieving remission with medical therapy usually requires many years of treatment, the risk for adverse reactions is relatively high and relapse is common after medication discontinuation. So there is debate about how Graves disease should be treated in children. The aim of this study was to identify predictors of remission after 2 years of antithyroid medication use. Methods. We retrospectively studied children who had Graves disease and were treated with antithyroid medication. We compared children who achieved remission after 2 years with those who had persistent disease to determine which variables were associated with remission. Results. Forty-one patients were enrolled, of which $5(12.2 \%)$ achieved remission and $36(87.8 \%)$ could not achieve remission until the last visit. There were no significant differences in T3, free T4, antimicrosomal antibody (AMA), and TSH-binding inhibitory immunoglobulin (TBII) between the two groups at presentation. However, the remission group had significantly higher titer of TSH than did the no-remission group at presentation $(0.11 \pm 0.04$ versus $0.04 \pm 0.04 \mathrm{mIU} / \mathrm{L}, P=.04)$. And the remission group had significantly lower titer of antithyroglobulin antibody (ATA) than did the no-remission group (189.6 \pm 103.2 versus $520.4 \pm 677.1 \mathrm{U} / \mathrm{ml}, P=.01)$. The no-remission group had higher ATA concentrations after 3 months of treatment with antithyroid medication than did the remission group $(583.3 \pm 763.9 \mathrm{U} / \mathrm{ml}$ versus $211.2 \pm 147.0 \mathrm{U} / \mathrm{ml}, P=.02)$. Conclusion. TSH, ATA concentrations at presentation and ATA concentration after 3 months of treatment can be predictors of remission in children with Graves disease after 2 years of antithyroid medication use.

\section{Long-Term Followup Study of Newborns with TSH Elevation without Low T4 in Neonatal Screening}

\section{Su-Young Kim and Sang-In Bae}

Pusan National University Children's Hospital, Yangsan, Korea

There have been few studies about long-term followup of babies with TSH elevation without low T4 in neonatal screening. We aimed to help diagnosis and treatment of those babies. We retrospectively reviewed medical records of 16 male and 26 female neonates whose T4 was normal and TSH were elevated in neonatal screening. Mean T4 and TSH were $8.61 \pm 1.55 \mu \mathrm{g} / \mathrm{dl}, 49.66 \pm 36.56 \mu \mathrm{U} / \mathrm{dl}$ respectively. Untreated patients were fifteen in whom TSH titers were normalized 
within 1.5 months ( 8 patients), 3 months (5 patients), 6 months (1 patient), and 9 months ( 1 patient) of age. Twentyseven patients were treated with thyroid hormone, among whom 11 patients were oldes than 3 years of age at the study point. Among those 11 patients, 6 patients could stop thyroid hormone therapy after trial off, but 5 are still on the thyroid hormone replacement. Those 5 patients had ectopic thyroids ( 4 patients) or hypoplastic thyroid ( 1 patient), all of who had been detected at radionuclide thyroid scan done at neonatal period. In conclusion, in many patients with hyperthyrotropinemia without hypothyroxinemia, TSH level become normalized within 9 months of age, although we should wait for more studies about long-term prognosis of those without hormone replacement. Radionuclide thyroid scan would be helpful to decide whether patients really need hormone replacement therapy.

\section{Seizure and Encephalopathy Associated with Thyroid Storm in Children}

\section{Hae Sang Lee and Jin Soon Hwang \\ Department of Pediatrics, Ajou Unviersity School of Medicine, Ajou University Hospital, Suwon, Korea}

Objective. Thyroid storm is a rare occurrence in children. The occurrence of seizures with thyroid storm is very rare. The aim of the study was to evaluate three cases of thyroid storm in patients who presented with seizures. Methods. We retrospectively reviewed the medical records of 46 hyperthyroidism patients who were hospitalized at our pediatric unit between 2003 and 2009. We report the clinical and laboratory findings of three patients with thyroid storm who had a seizure in the absence of a history of neurologic disease. Results. Case 1 was an 11-year-old girl who presented with a headache, abdominal pain, diarrhea, vomiting, and fever. Three days after admission, she suffered two generalized tonic-clonic seizures lasting for about 30 seconds and five minutes, respectively. Case 2 was a 10 -year-old boy who presented with fever, palpitation, and irritability. On the day of admission, he had tonic-clonic movements for about five minutes. Case 3 was a 14-year-old boy who was diagnosed with hyperthyroidism one year prior to admission. However, he was not taking antithyroid drugs. He was admitted with a complex partial seizure that lasted for about 10 minutes. The serum thyroid hormone levels of all patients were elevated. Acute medical management with propylthiouracil, Lugol's solution, hydrocortisone, and propranolol led to complete resolution of the symptoms. Conclusion. Patients with thyroid storm may be predisposed to the development of neuropsychiatric change. Early recognition and multidrug treatment of thyroid storm are essential to reduce morbidity and mortality from this disorder.

\section{Relationship of Congenital Hypothyroidism and TSH Receptor Gene Mutation and Clinical Phenotype}

Liu Xiaa, Liu Xiaohonga, and Wang Shayanb
Shenzhen Children's Hospital 518 026,

Shenzhen People's Hospital 518000

Objective. Congenital Hypothyroidism is one of the most common pediatric endocrine diseases and congenital metabolic defects. It is one of the main causes of mental retardation of children. Relationship of congenital hypothyroidism and thyroid-stimulating hormone receptor (TSHR) gene mutation has been studied in order to explore the molecular biology basis of thyroid disease. of hypothyroidism and the relationship between hypothyroidism and TSHR gene mutation has been studied by detecting gene mutations of exon 10 and exon 6 on TSHR. The molecular mechanism of congenital $\mathrm{CH}$ has been further clarified. Methods. Extracting DNA of white blood cells from 40 congenital hypothyroidism patients diagnosed in our hospital, and enlarging its TSHR gene primers. Compare the sequences obtained with the standard sequence of human TSHR gene in the Gene Bank and test their sequences. Results. Hypothyroidism patients have been examined by color Doppler, among which 8 patients have been examined by isotope scanning. Among 40 hypothyroidism patients, 14 cases $(35 \%)$ are thyroid hypoplastic, 8 cases $(20 \%)$ are thyroid absent, 6 cases (15\%) are thyroid enlarged, 5 cases $(12.5 \%)$ are thyroid Cystic, 4 cases $(10.5 \%)$ are thyroid ectopic, and 3 cases $(7.5 \%)$ are with normal thyroid. Results Positive and negative sequence test results of TSHR gene mutation: Codon 727 of hypothyroidism patients numbered $1,2,3,4,5,6,10,11,14,15,16,17,18,20,21,22,24,25$, 26,27 has been changed from GAC to GAG; 727 amino acid substitution: Asp72 $\rightarrow$ Glu, D727E homozygous mutation, while the hypothyroidism patients number $7,8,9,12,13$, 19, 23 are heterozygous mutations. Conclusion. TSHR gene mutation of $27 \mathrm{CH}$ patients has been studied and it was found that the TSHR 727 codons have changed from GAC to GAG, 727 amino acid Asp727 have been substituted to Glu, among which 20 were homozygous mutants, 7 were heterozygous mutation. The results further elucidate the molecular mechanism of congenital $\mathrm{CH}$ and provides a new way of thinking.

\section{An Echocardiographic Study of Cardiac Changes in Congenital Hypothyroidism and the Response to Treatment}

\section{Shanshan Mao, Zhefeng Yuan, and Chaochun Zou \\ Department of Endocrinology, The Children's Hospital of Zhejiang University School of Medicine}

Congenital hypothyroidism $(\mathrm{CH})$ is a common endocrine disease of pediatrics, which results in severe mental retardation and growth failure as well as other functional impairment because of the deficiency of thyroid hormone during human perinatal development. Recent research has demonstrated that cardiac function is impaired in children with $\mathrm{CH}$, which can be fully or partly reversed by levothyroxine (L-T4) substitution therapy. However, only few, contradictory data are available on cardiac function in 
neonates with $\mathrm{CH}$. The aim of this study was to investigate the effects of L-T4 substitution therapy on left and right ventricular function in neonates with $\mathrm{CH}$, measured with Quantitative tissue velocity imaging (QTVI) and pulsedwave Doppler echocardiography (PWD). Fifty full-term neonates with $\mathrm{CH}$ aged 17 28 days before and after 1 month of levothyroxine substitution therapy as well as 35 normal neonates aged 17 28 days underwent PWD and QTVI. On QTVI, peak mitral and tricuspid annular systolic (S) velocity, peak early (E) and late (A) mitral and tricuspid annular diastolic velocity were measured, and the E/A ratio was calculated. All the biochemical tests and echocardiographic evaluations were reevaluated after 1 month of replacement therapy. In $\mathrm{CH}$ neonates, QTVI showed that $\mathrm{CH}$ neonates had significantly lower S, E, and E/A of both left and right ventricles $(P<.001$, resp.). However, A of left and right ventricles did not differ significantly. After 1 month of substitutive therapy, $\mathrm{CH}$ neonates showed a significant increase of $\mathrm{S}$ and $\mathrm{E}$ and a subsequent increase of the $\mathrm{E} / \mathrm{A}$ ratio $(P<.001$, resp. $)$. In conclusion, our findings demonstrated that $\mathrm{CH}$ is associated with impaired left systolic and diastolic function in addition to impaired right systolic and diastolic function. Early L-T4 replacement therapy should be advised for these neonates with the aim to correct preclinical cardiac dysfunction.

\section{Near-Fatal Uterine Bleeding in a Congenital Hypothyroid Adolescent}

\section{Catherine Anne G. Pangilinan}

\section{University of the Philippines, Philippine General Hospital}

Abnormal uterine bleeding is an infrequent but serious complication of inadequately treated hypothyroidism. This is a case of a 16-year-old girl who was brought to the emergency room because of seizure. The patient is a diagnosed case of Congenital Hypothyroidism since one month of age by newborn screening. She is maintained on levothyroxine but with poor compliance because of financial constraints. She presented with one week history of menorrhagia consuming five to six fully soaked pads per day associated with pallor and generalized body weakness. Five days after the onset of menses, she had four episodes of generalized tonic-clonic seizures, each lasting for 30 seconds, which prompted the consultancy. On admission, she was lethargic, clammy, and hypotensive with BP: 60/40 mmHg, CR: 140/minutes, RR: 40/minutes and with poor pulses. Neurologic exam was normal. ABG showed metabolic acidosis. Oxygen supplementation and initial fluid resuscitation were done. Plasma expanders, dopamine and dobutamine were likewise started with gradual improvement of vital signs. CBC showed severe anemia (Hgb: $32 \mathrm{~g} / \mathrm{dl}$, Hct: 0.09) warranting pRBC transfusion. Transabdominal ultrasound revealed hematometra with thinned-out endometrium. Conjugated estrogen, ferrous sulfate and tranexamic acid were likewise given. On further examination of the patient, she was noted to be severely stunted (Wt: $30 \mathrm{~kg}$ and $\mathrm{Ht}: 120 \mathrm{~cm}$, both $<\mathrm{P} 5$ ), with periorbital edema, depressed nose bridge, underdeveloped breasts and genitalia (Tanner Stage 2) and very dry skin. The parents claimed that the patient is also mentally-retarded. Thyroid function tests were consistent with hypothyroidism (TSH: >100, NV: 0.3-3.8 miu/L; FT4: 5.6, NV: $11-24 \mathrm{pmol} / \mathrm{L})$. L-thyroxine at $100 \mathrm{mcg} /$ day was started. There was a decrease in the menstrual blood flow noted on the 3rd hospital day, with cessation of the bleeding on the 5th day. There was no recurrence of the seizure and vital signs stabilized. The patient was gradually weaned from inotropes and IV fluids and was subsequently discharged improved.

\section{Thyrotoxic Hypokalemic Paralysis: A Case Report}

\section{Catherine Anne G. Pangilinan}

\section{University of the Philippines, Philippine General Hospital}

Thyrotoxic Hypokalemic Paralysis is an uncommon endocrine emergency which presents as recurrent suddenonset muscle weakness associated with hypokalemia in a hyperthyroid patient. Although the exact mechanism is still unclear, thyrotoxicosis is thought to alter the plasma membrane permeability to sodium and potassium, a function linked to the Na+-K+ ATPase activity. There is a strong male preponderance, more commonly affecting hyperthyroid patients of Asian descent. Most reported cases present between ages of 20 and 39 years. This is a case of a 17-year-old male who was brought to the ER with a chief complaint of inability to ambulate. He is a diagnosed case of Graves' Disease since three months prior to consult with initial presentation of gradually-enlarging anterior neck mass. He was started on methimazole but was discontinued two weeks ago due to financial constraints. He presented with one-week history of progressive weakness of both lower extremities. This was associated with palpitations, hyperdefecation (at least 3 bowel movements/day), heat intolerance, and tremors. A day prior to admission, he was unable to rise from bed and to ambulate. He came in wheelchair-borne, agitated with BP: $100 / 60 \mathrm{~mm} \mathrm{Hg}$, CR: 130/minutes, RR: 25/minutes, afebrile. Pertinent PE findings include exophthalmos, diffusely enlarged thyroid gland and tremors. The heart, lungs and abdomen were essentially normal. Neurologic examination revealed symmetrical proximal muscle weakness associated with tenderness, more prominent on the lower extremities. There was no atrophy or fasciculation of the involved muscles. Laboratory workups showed hypokalemia $(2.4 \mathrm{mmol} / \mathrm{L}$, $\mathrm{NV}$ : $3.5-5.10 \mathrm{mmol} / \mathrm{L})$. $\mathrm{CBC}$ and other electrolytes were within the normal range. ECG showed sinus tachycardia, ST segment changes and presence of $U$ waves. Thyroid function test revealed a hyperthyroid state (TSH: $0.26, \mathrm{NV}$ : 0.39-6.0 uiu/mL; FT4: 8.21, NV: 0.8-2.0 ng/dl). Assessment made was Thyrotoxic Hypokalemic Paralysis. IV correction of hypokalemia was initiated. Methimazole and propranolol were given. There was significant clinical improvement after one day. Repeating serum potassium was within 
normal range $(3.5 \mathrm{mmol} / \mathrm{L})$. The patient was discharged improved and able to walk. He was advised to continue methimazole. Repeating TSH and FT4 done after one month showed normal values $(0.33 \mathrm{uiu} / \mathrm{ml}$ and $1.36 \mathrm{ng} / \mathrm{dl}$, resp.). The patient has then lost to followup. After five months, he was readmitted for muscle weakness and difficulty ambulating. He also complained of palpitations and difficulty in breathing. He claimed that methimazole was discontinued for more than a month. Blood tests again showed hypokalemia. He subsequently improved after being given potassium and resuming methimazole and propranolol.

\section{Pediatric Primary Thyroid Carcinoma: An Institutional Experience in India}

\author{
Leena Priyambada, ${ }^{1}$ Anna Simon, ${ }^{1}$ Sudipta Sen, ${ }^{2}$ \\ Jacob Chacko, ${ }^{2}$ and Aravindan Nair ${ }^{3}$ \\ ${ }^{1}$ Division of Pediatric Endocrinology, Department of \\ ${ }^{2}$ Pediatric Surgery \\ ${ }^{3}$ Endocrine Surgery; Christian Medical College, \\ Vellore, India
}

Thyroid cancer is quite rare in the pediatric age group, even though it is the most common endocrine malignancies. The incidence of these malignancies is increasing over the years. We describe here the clinical presentation, course and factors associated with disease outcome in children and adolescents with primary thyroid carcinoma. Methods. Data of all patients younger than 18 years presenting with primary thyroid carcinoma between 2003 and 2009 were analysed retrospectively. Results. Thirty-one patients at a mean age of diagnosis of $13.5+3.2$ years were included in the study. A female predominance (64\%) was seen, especially in patients older than 10 years. None of the patients (except for medullary carcinoma with a family history of MEN2) had a suggestive risk factor. More than $90 \%$ of patients presented with a painless thyroid swelling (median duration of 6 months). The regional lymph nodes were involved in $64 \%$ of patients. Distant spread (asymptomatic pulmonary metastases) was seen in $13 \%$. All patients were euthyroid. Preoperative FNAC was either false negative or inconclusive in 55\% of the cases. Histopathologically, the majority were papillary (61\%), 25\% were follicular variant of papillary, $10 \%$ were follicular and 3\% were medullary carcinomas. Associated thyroiditis on histopathology was seen, especially in patients with recurrent disease. Thyroid antibodies were positive in only one of the 15 cases done. Surgery was the initial treatment modality in all patients. Total thyroidectomy was done for all patients, with additional modified radical neck dissection in 35\% of the cases. Residual malignancy on postoperative iodine scan was seen in $>90 \%$ of the cases. $80 \%$ underwent Iodine ablation. $13 \%$ of patients received external beam radiotherapy for extensive regional metastasis. The followup duration ranged from 6 to 84 months in $90 \%$ of the patients. Recurrence, commonly nodal, was seen in $16 \%$ of patients. In $25 \%$ patients disease persisted, commonly evidenced by elevated thyroglobulin or as persisting lung metastases. Persistence of disease was associated with a male predominance $(62 \%)$, lymph node and lung metastases, papillary tumors, multicentricity and extrathyroidal extension on biopsy. Patients with follicular variant of papillary carcinoma had a favourable outcome. Conclusion. Pediatric primary thyroid carcinoma commonly presents as asymptomatic neck swelling and nodal metastases. Absence of risk factors and lack of sensitivity of FNAC adds to delay in diagnosis. A high incidence of residual malignancy necessitates postoperative iodine scan in all patients. More than a third of the patients have either recurrence or persistence of disease, hence prolonged careful follow up is necessary. Male gender, multicentricity, extrathyroidal extension on histopathology, and lung metastases may be associated with worse outcome.

\section{Thyroid Hormone Receptor Gene Analysis on Two Chinese Families with Resistance to Thyroid Hormone}

\author{
Qian Dong, Chun-xiu Gong, Yi Gu, and Chang Su
}

\author{
The Capital Medical University, \\ Beijing Children's Hospital, Beijing 100045, China
}

Background. Resistance to thyroid hormone (RTH) is a dominantly inherited syndrome of reduced tissue responsiveness to thyroid hormone usually due to mutations located in the ligand-binding domain and adjacent hinge region of the thyroid hormone receptor $\beta$ (TR $\beta)$. Objective. We describe the clinical and laboratory characteristics and the genetic analysis of patients with this rare disorder from two Chinese children and their families. Methods. We detected the thyroid function test (TFT), the complete blood cell count (CBC), blood biochemistry markers, magnetic resonance imaging scan (MRI) of pituitary and electrocardiogram (ECK) for the two patients. We also detected the TFT of their family members as well. Direct DNA sequencing of the TR $\beta$ gene was done for people with abnormal TFT. Results. All the two RTH children had goiter, associated with irritability, aggressiveness, and profuse sudoresis, but only one had proptosis. The TFT showed high levels of circulating freethyroid hormones (FT4 and FT3) and normal- or high-TSH concentrations. Other blood biochemistry examinations were almost normal. All the two children used bromocriptine and the clinical presentations got improved obviously and no advert effect. We identified a novel missense mutation, A317D, located in exon 9 of the gene in one child and his mother, but his mother showed no clinical presentation. The other child and his parents were found no mutation in the TR $\beta$ gene. Conclusion. The present report shows that there is a novo mutation found in the $\operatorname{TR} \beta$ in one case and his mother. The other child with RTH found no mutation. And this research verified the phenomena that there is a heterogeneous within the same mutation of RTH patient. 


\section{Newborn Thyroid Screening in a Tertiary Care Hospital in South India Involving a Large Birth Cohort}

\author{
Anna Simon, Palani Raghupathy, A. K. Jana, \\ F. S. Geethanjali, and Sarah Mathai \\ Departments of Child Health and Clinical Biochemistry, \\ Christian Medical College, Vellore, South India
}

Background. Neonatal thyroid screening is part of the national screening programme in most developed countries, but not as yet available in developing countries. Early diagnosis and early treatment can prevent most of the consequences of congenital hypothyroidism $(\mathrm{CH})$. Methods. All babies $>26$ weeks gestational age born between July 2001 and June 2009 with cord blood TSH $>25 \mathrm{mIU} / \mathrm{ml}$ were recalled for repeating thyroid function tests after 72 hours. Primary hypothyroidism was confirmed based on elevated TSH levels and low FT4 values. Tc99m thyroid nuclear scan was done in all babies with primary hypothyroidism confirmed on blood tests. Perchlorate discharge test was done in babies with suspected dyshormonogenesis. Results. 63,853 samples of cord blood TSH were collected. Of the 1718 samples which were repeated 52 were confirmed to have $\mathrm{CH}$. The mean recall rate was $2.7 \%$. Missed repeat samples $(1 \%)$ were due to either neonatal deaths or failure of parents to return for repeat test. Congenital hypothyroidism was confirmed in 52 babies and the hospital based incidence was 1: 1228 . There was no female preponderance (52\%). $38.5 \%$ of those confirmed with $\mathrm{CH}$ had thyroid dyshormonogenesis, $27 \%$ agenesis, $15.5 \%$ hypoplasia and (15.5\%) ectopia. Transient hypothyroidism was diagnosed in 1 of the 52 confirmed cases. 5 babies were diagnosed with central hypothyroidism who had very low initial cord blood TSH $(<0.1 \mathrm{mIU} / \mathrm{ml})$ and on repeated sampling had low TSH and FT $4.52 \%$ of babies with confirmed $\mathrm{CH}$ had treatment initiated within the first week, $27 \%$ within 2 weeks and $21 \%$ within 4 weeks after birth. Conclusion. The incidence of congenital hypothyroidism in this study is higher than most reported studies. High incidence rates for $\mathrm{CH}$ reported in this hospital-based study suggest the need for a national programme in India for screening for $\mathrm{CH}$. Cord blood TSH remains the preferred screening method in developing countries where most mothers and babies are discharged within a day after delivery.

\section{Multicystic Ovaries due to Hypothyroidism: A Case of Van Wyk-Grumbach Syndrome}

\author{
Rashida Vasanwala, ${ }^{1}$ Fabian Yap, ${ }^{1}$ Jeremy Ho, ${ }^{2}$ \\ and Ong Lin Yin ${ }^{2}$ \\ ${ }^{1}$ Department of Paediatric Endocrinology, \\ KK Women's and Children's Hospital, Singapore \\ ${ }^{2}$ Department of Paediatric Surgery, \\ KK Women's and Children's Hospital, Singapore
}

Background. Ovarian hyperstimulation is a recognized complication of longstanding hypothyroidism. In 1960 Van Wyk and Grumbach described a syndrome of juvenile hypothyroidism, precocious puberty and ovarian enlargement. Several theories have been proposed to explain precocious puberty caused by hypothyroidism. Van Wyk and Grumbach suggested increased pituitary production of not only thyroid stimulating hormone but also follicle-stimulating and luteinizing hormones and prolactin when there was no negative feedback from thyroid hormones. The mechanism was dubbed "hormonal overlap in pituitary feedback." The current most widely accepted theory is that high levels of thyroid-stimulating hormone act on follicle-stimulating hormone receptors because of molecular similarities between the two hormones, This mechanism, known as "specificity spill over" has been demonstrated in vitro. Ovarian stimulation in the hypothyroid child may result in oestrogen production, breast development, endometrial proliferation and vaginal bleeding. These findings undergo complete regression with thyroid hormone replacement therapy. Objective. To describe a case of bilateral large multicystic ovaries in a young child who presented to paediatric surgical unit and underwent left salphingo-oophorectomy and right ovarian cystectomy. The case was a possible diagnostic dilemma, with subsequent diagnosis of primary hypothyroidism. Methods. We present the clinical, radiological, biochemical and histopathological findings in a patient with megaovaries who underwent unilateral oophorectomy. Result. The patient, 5-years-old girl presented with per-vaginal bleeding, abdominal distension and abdominal pain. The CT scan of the abdomen showed bilateral adnexal masses likely ovarian in origin and neoplastic in nature given their size and complexity. The surgical intervention was a consequence of torsion of left megaovary necessitating oophorectomy. Histopoathological report commented that both ovaries contained large luteinized follicular cysts with haemorrhagic infarction of left tube and ovary with no viable tissue. Endocrinological evaluation showed that there was no history suggestive of hypothyroidism. Examination revealed prominent nippleaerolar complex but neither breast buds, nor signs of virilisation and cliteromegaly. Patient was clinically euthyroid. Her gonadotropin response to LHRH was prepubertal, she had elevated levels of oestradiol, prolactin and CA-125, with normal levels of alpha fetoprotein and beta-HCG. The thyroid function test revealed severe primary hypothyroidism with very high TSH levels. She was started on thyroid hormone replacement. Conclusion. The association of primary hypothyroidism with cystic ovarian enlargement and precocious puberty is important to recognize. In the absence of suspected torsion, surgery is unnecessary, as cyst regression occurs after appropriate thyroid hormone replacement.

\section{Significance of Bone Age Inspection in Children with Hypothyroidism} Yan Xiaoli, Wang Zhihua, Li Xiaoqing,
and Pan Qiuhong

\section{Xi'an children's hospital, Xi'an 710003, China}

Objective. To observe the bone age development in children with hypothyroidism and explore the potential applications 
in diagnosis and therapy of hypothyroidism. Methods. We observed 37 children's X-ray films of left hands and wrists and applied method for assessing bone age of Chinese children to score their bone age. Results. The bone age of children with hypothyroidism is younger than their chronological age and the bone age caught up the chronological age after treatment in the followed 3 years. Conclusions. Bone age inspection has important instructional significance in diagnosis and treatment of hypothyroidism in children.

\section{Clinical Significance of Measurements of Serum Gh and Igf- 1 in Children with Congenital Hypothyroidism}

\author{
Xiaoli Yan, Zhihua Wang, Xiaoqing Li, \\ and Qiuhong Pan
}

\section{Xi'an children's hospital, Xi'an 710003, China}

Objective. To detect the serum growth hormone (GH), insulin-like growth factor-1(IGF-1) levels in children with congenital hypothyroidism $(\mathrm{CH})$ and explore the change regulation of GH-IGF axis and thyroxine. Methods. Serum levels of GH, IGF-1 T3, T4, FT3, FT4 and TSH of 30 children with $\mathrm{CH}$ were detected before and after levothyroxine sodium treatment by means of chemiluminescence immunoassay and 28 normal children were the control group. Results. Serum GH, IGF-1 levels in children with $\mathrm{CH}$ were lower, and there were statistical significance compared with that of control group. The serum GH, IGF-1 levels were normal after thyroxine substitute therapy, in addition, there were statistical significance between before and after treatment. Conclusions. The GH-IGF axis is insufficient in $\mathrm{CH}$ children, which is one of the major reasons for stature short. Early thyroxine substitute medication helps children with $\mathrm{CH}$ to maintain normal growth and development.

\section{Analysis of the Behavior Problem and Interference Factors in Preschool Children with Early-Treated Congenital Hypothyroidism}

\section{Zhang- liqin and Fu Ping Zhou ji-yong \\ Qingdao Women and Children's Medical Health Center, Qingdao 266011, Shandong, China}

Objective. To evaluate behavior Problem and interference factors in preschool children with early-treated congenital hypothyroidism $(\mathrm{CH})$, reduce the behavior problem so as to improve living quality of $\mathrm{CH}$. Methods. A historical cohort study was performed in 4-6 years old children with $\mathrm{CH}$ detected by neonatal screening in Qingdao. 47 children with permanent $\mathrm{CH}$ were studied and compared to 58 matched normal children born at the same time. Behavior problem of the children was evaluated on the Achenbach Child Behavior Checklist (CBCL), The related factors of the two groups were investigated by self-made questionnaires, children temperament assessment battery, Family
Environment Scale-Chinese Version (FES-CV) respectively. Results. In preschool, the behavior problem incidence of $\mathrm{CH}$ children was higher than normal children. The type of temperament, and family environment were important influencing factors in $\mathrm{CH}$. Conclusion. Though $\mathrm{CH}$ children have been early-diagnosed and early-treated, the present findings strongly suggest that the behavior problem incidence of $\mathrm{CH}$ children was higher than normal children, psychological assessment and consulation must be done so as to they could grow healthy.

\section{Adrenal Disorders}

\section{Growing Numbers of Children with CAH in Vietnam?}

\author{
Vu Chi Dung, ${ }^{1}$ Bui Phuong Thao, ${ }^{1}$ \\ Khu Thi Khanh Dung, ${ }^{1}$ Can Thi Bich Ngoc, ${ }^{1}$ \\ Nguyen Ngoc Khanh, ${ }^{1}$ Nguyen Thi Hoan, ${ }^{1}$ \\ Nguyen Thanh Liem, ${ }^{1}$ Kate Armstrong, ${ }^{2}$ \\ Maria Craig, ${ }^{3}$ and Garry Warne ${ }^{4}$
}

\author{
${ }^{1}$ National Hospital of Pediatrics, Hanoi \\ ${ }^{2}$ CLAN (Caring \& Living As Neighbours) \\ ${ }^{3}$ The Children's Hospital at Westmead, Sydney \\ ${ }^{4}$ Royal Children's Hospital Melbourne
}

Objectives. The National Hospital of Pediatrics (NHP) in Hanoi is an 850 bed tertiary referral centre servicing approximately 30 million people from Northern provinces of Vietnam. Congenital Adrenal Hyperplasia (CAH) was first diagnosed in Hanoi in 1976. This audit was undertaken to analyze anecdotal reports of increasing patient numbers. The incidence of CAH in Vietnam is not known currently, although pilot Newborn Screening (NBS) trials are continuing in HCMC. Methods. Retrospective review of all CAH patients registered at NHP from 1999-2009. Ethical clearance was granted by the NHP Directorate. Results. At the start of 1999 there were 71 children with CAH managed at NHP. By December 2009 this increased to $494(47 \%$ male and $53 \%$ female; $72 \%$ salt wasting $\mathrm{CAH}$ ), representing a more than five fold increase over 11 years. Number of new cases doubled from 30 to 60 in 2009. Most children (72\%) were diagnosed at less than 12 months of age (39\% at less than 1 month of age); $70 \%$ $(348 / 494)$ of all children were younger than 10 years. Formal mortality figures were low (6 known deaths), although loss to follow-up unknown. There are data to suggest persisting mortality from undiagnosed CAH (evidenced by low ethnic minority group representation; few children from remote provinces; higher average income of CAH families; gender ratio shift; reports of sibling deaths). Patient to paediatric endocrinologist (494:6) ratios at NHP are very high compared with higher income countries. Conclusions. The caseload of CAH at NHP has increased since 1999 and additional capacity is needed for patient care given high patient to staff ratios. Whilst initiatives such as: affordable access to medication; introduction of 17-OHP testing; translation of educational materials; and resource 
nurse training to promote family education have focused on improving quality of life for children living with $\mathrm{CAH}$ in Vietnam, objective clinical benchmarks to track progress over time are required. Introduction of NBS would enable more accurate estimation of $\mathrm{CAH}$ incidence, reduce infant mortality and minimize trauma to affected infants and their families.

\section{Screening for Mutations in DAX1 (NR0B1) and SF1 (NR5A1) Gene in 16 Boys with Primary Adrenal Failure}

\section{Guoying Chang, Zhiya Dong, Wei Wang, and Defen Wang}

\section{Department of Paediatrics, Ruijin Hospital, School of Medicine, Jiaotong University, Shanghai, China}

Context. Primary adrenal failure is a life-threatening condition that can be caused by a range of etiologies, including autoimmune, metabolic, and developmental disorders. The nuclear receptors DAX1 (NR0B1) and steroidogenic factor1 (SF1, NR5A1) play an important role in adrenal development and function, and mutations in these transcription factors have been found in patients with adrenal hypoplasia. Objective. To investigate the prevalence of DAX1 and SF1 gene mutations in 16 primary adrenal failure male patients associated or not with hypogonadism and/or disorder of sex development. Subjects. A total of 16 boys were diagnosed as primary adrenal failure of unknown etiology characterized by their clinical manifestations (except one associated with hypospadia). The age at first presentation was from newborn to 10 years old (average $4.5 \pm 3.9$ years). The diagnosis was confirmed by laboratory findings, in addition with the genetic studies to assess the prevalence of DAX1 and SF1 gene mutations in our group. The patients ranged in age from one to $25 \mathrm{yr}$ in the last follow-up, of which, 9 cases under puberty, 2 cases with normal sexual development, and 5 cases with puberty delayed. Replacement treatment with glucocorticoid was maintained in all the patients. Methods. Genomic DNA (from peripheral blood leukocytes) was obtained from all the patients and their related suspected family members. The full coding regions and intron-exon boundaries of DAX1 (exon1, 2) or SF1 gene (exon2-7) were amplified with PCR, then directly sequenced. DAX1 gene mutations were screened for all the patients and those who were negative undergone SF1 gene screening. Results. (1) Genetic screening: Five frameshift mutations (304delC, R98E; 341342delCT, S111X; 746delG, R244L; 663-664delCA, T218E; 853delG, W253C), one missense mutation (T785C, L262P, two patients) and one nonsense mutation $(424 \rightarrow \mathrm{T}, \mathrm{E} 137 \mathrm{X})$ were found in DAX1 gene, and only one mutation identified in SF1 gene $(624 \rightarrow \mathrm{G}, \mathrm{G} 146 \mathrm{~A})$. The mutations underlined were novel and the others have been reported previously. (2) Prevalence of DAX1 gene mutations was 50\% $(8 / 16)$ in our patients, 5 of the positive cases (5/8) show positive family history verified by genetic studies, and 4 cases (4/8) with under developed puberty. SF1 gene mutation occurred in the patient with hypospadia. Conclusions. DAX1 genetic defects are a relatively frequent cause of primary adrenal failure while SF1 gene mutations relatively rare and more likely to be associated with disorders of sexual development.

\section{Update of Clinical Phenotype and Mutation Database: Identification of a Novel Mutation of the StAR Gene in Korean Patients with Congenital Lipoid Adrenal Hyperplasia}

\author{
Jin-Ho Choi, ${ }^{1}$ Hye-Young Jin, ${ }^{1}$ Beom-Hee Lee, ${ }^{2}$ \\ Gu-Hwan Kim, ${ }^{2}$ Jung Hyun Lee, ${ }^{3}$ Hae Soon Kim, ${ }^{4}$ \\ Jeh-Hoon Shin, ${ }^{5}$ Choong-Ho Shin, ${ }^{6}$ \\ Young Jong Woo, ${ }^{7}$ Jee Suk Yu, ${ }^{8}$ Dae-Yeol Lee, ${ }^{9}$ \\ Yong Hoon Jun, ${ }^{10}$ and Han-Wook Yoo ${ }^{1,2}$
}
${ }^{1}$ Division of Pediatric Endocrinology and Metabolism, Department of Pediatrics,
${ }^{2}$ Medical Genetics Clinic and Laboratory, Asan Medical Center Children's Hospital, University of Ulsan College of Medicine, Seoul, Korea ${ }^{3}$ Department of Pediatrics, Kosin University College of Medicine, Busan, Korea
${ }^{4}$ Department of Pediatrics, College of Medicine, Ewha Womans University, Seoul, Korea ${ }^{5}$ Department of Pediatrics, College of Medicine, Hanyang University, Seoul, Korea
${ }^{6}$ Department of Pediatrics, College of Medicine, Seoul National University, Seoul, Korea
${ }^{7}$ Department of Pediatrics, College of Medicine, Chonnam National University, Kwangju, Korea ${ }^{8}$ Department of Pediatrics, College of Medicine, Dankook University, Cheonan, Korea
${ }^{9}$ Department of Pediatrics, Chonbuk National University Medical School, Jeonju, Korea
${ }^{10}$ Department of Pediatrics, College of Medicine, Inha University, Incheon, Korea

Purpose. The steroidogenic acute regulatory (StAR) protein plays a crucial role in steroidogenesis, as it promotes the transfer of cholesterol from outer to inner mitochondrial membrane. Mutations in the gene encoding StAR cause congenital lipoid adrenal hyperplasia (CLAH), a disease that is the most severe form of congenital adrenal hyperplasia characterized by a deficiency of adrenal and gonadal steroid hormones. In this study, we updated the clinical characteristics and mutation spectrum of the StAR gene in Korean patients with CLAH. Methods. Seventeen unrelated Korean patients were referred to Medical Genetics Clinic and Laboratory, Asan Medical Center for molecular analysis of the StAR gene. Clinical features (skin hyperpigmentation, external genitalia, salt-losing phenomenon, karyotype, adrenal imaging, etc.) and endocrine data (ACTH, renin, 17-OHP, DHEA-S, and adrenal androgen) were collected retrospectively. After obtaining informed consent, genomic DNA was extracted from peripheralblood leukocytes. Seven exons of the StAR gene and their intronic flanking sequences 
were amplified by PCR, and subsequently sequenced using ABI3100 Genetic analyzer. Results. All patients developed hyperpigmentation and adrenal insufficiency in the neonatal period. Ten out of 17 patients were genetically males, although they had female external genitalia. The basal ACTH levels and plasma renin activity were extremely high in all patients. Fourteen patients were homozygous for the p.Q258X mutation, and three patients were compound heterozygotes of p.Q258X/p.L98R, p.Q258X/p.A218V, and p.A218V/c.745-6_810del. The p.Q258X mutant allele was identified in 30 out of 34 alleles $(88.2 \%)$. The c.653C $>\mathrm{T}$ (p.A218V) mutation was detected in 2 out of 34 alleles $(5.9 \%)$. The p.L98R and c.745-6_810del mutations were found in one out of 34 alleles (2.9\%), respectively. Conclusions. We identified four different mutations in the StAR gene. Three mutations have been previously reported and one mutation is a novel mutation (c.7456_810del). As previously reported, the p.Q258X mutation in the StAR gene is the most common in Korean population by a founder effect. In vitro expression analysis of the novel mutation and long-term follow-up should be needed for evaluation of genotype-phonotype correlation.

\section{Testicular Adrenal Rest Tumors in a Patient with Untreated Congenital Adrenal Hyperplasia}

\author{
Hye Young Jin, ${ }^{1}$ Han-Wook Yoo, ${ }^{1,2}$ Gu-Hwan Kim, ${ }^{2}$ \\ and Jin-Ho Choi ${ }^{1}$

\section{${ }^{1}$ Division of Pediatric Endocrinology and Metabolism, Department of Pediatrics, \\ ${ }^{2}$ Medical Genetics Clinic and Laboratory, Asan Medical Center Children's Hospital, University of Ulsan College of Medicine, Seoul, Korea}

Background. Testicular adrenal rest tumors (TARTs) are considered aberrant adrenal tissue that has become hyperplastic because of elevated adrenocorticotropic hormone (ACTH) in male patients with congenital adrenal hyperplasia (CAH). Case. A 6-year-old boy presented with testicular enlargement, pubic hair. His height and weight were $124 \mathrm{~cm}$ (2.31 SDS) and $27 \mathrm{~kg}$ (2.63 SDS), respectively. The volume of each testes was $7 \mathrm{~mL}$, the stretched penile length was $7 \mathrm{~cm}$, and his bone age was 13 years by Greulich and Pyle's method, which was remarkably advanced for his chronological age. His serum 17-hydroxypregnenolone (17OHP) and testosterone levels were elevated to $480 \mathrm{ng} / \mathrm{mL}$ (normal range: $0.32-3 \mathrm{ng} / \mathrm{mL}$ ) and $2.0 \mathrm{ng} / \mathrm{mL}$ (normal range: 0.03-0.1 ng/mL), respectively. He was diagnosed with CAH complicated by precocious puberty. However, he was not followed-up. At age 17 years, he visited the outpatient clinic because of testicular enlargement and short stature. His right and left testicles were $10 \times 6 \mathrm{~cm}$ and $7.5 \times$ $4.5 \mathrm{~cm}$, respectively. His height was $155.1 \mathrm{~cm}-2.90$ SDS). The diagnosis of $\mathrm{CAH}$ due to 21 hydroxylase deficiency was confirmed by mutation analysis of CYP21A2. Testicular biopsy showed large, polygonal, eosinophilic cells with round nuclei and prominent nucleoli, suggesting that the tumors were TARTs. He was treated with dexamethasone for 3 weeks and the tumors regressed. Dexamethasone was changed to prednisolone and $9 \alpha$-fludrocortisone, and reduced testis size has been maintained. Conclusion. In patients with $\mathrm{CAH}$, poor compliance with treatment increases plasma ACTH levels, accelerating the development of TART. Although TARTs are usually benign, they may obstruct the seminiferous tubule, leading to possible gonadal dysfunction. Early detection of TARTs is important in preventing irreversible testicular damage. Patients with $\mathrm{CAH}$ should be regularly examined by digital palpation and radiological methods such as ultrasonography, to detect small-sized tumors. We describe here a patient with untreated $\mathrm{CAH}$ who developed TARTs, which regressed with dexamethasone therapy.

\section{Adrenal Insufficiency Presenting with Recurrent Seizures in an Infant}

\section{Ansu Mali Joshi, Rajesh Khadgawat, VP Jyotsna, and A C Ammini}

\section{Department of Endocrinology, AlIMS, New Delhi, India}

Introduction. Primary adrenal insufficiency is an uncommon, but well recognized cause for hypoglycemia in infants. Presented here is case of a child who presented with seizures and hypoglycemia due to adrenal insufficiency. Baby J.K. was the first born child of a non consanguineous marriage. This was a full term normal vaginal delivery, conducted at home, birth weight was $2.1 \mathrm{~kg}$. $\mathrm{He}$ was apparently normal except for dark complexion. Few weeks later he started having seizures and investigations revealed hypoglycemia and elevated plasma ACTH $(>2000 \mathrm{pg} / \mathrm{ml})$. With hydrocortisone supplementation both seizures and hypoglycemia stopped. He is now 3 years old, developmental milestones are within normal limits. Schirmer's test was negative. CECT abdomen revealed normal adrenal glands. Common causes of adrenal insufficiency in neonates are congenital adrenal hyperplasia, Xlinked congenital adrenal hypoplasia, peroxisomal disorders, familial glucocorticoid deficiency, Allgrove syndrome or triple A syndrome (alacrima, achalasia, and adrenal insufficiency), and acquired conditions such as adrenal hemorrhage, trauma and infections. The present case is possibly a case of Familial Glucocorticoid Deficiency (FGD). FGD is an autosomal recessive disorder, first described in 1959, and since then about 50 cases have been reported from around the world including Caucasian, African-American, and East Indian populations. The age of presentation varies from birth to 9 years of age. Unlike classical Addison's disease, patients with FGD never develop mineralocorticoid deficiency. 


\section{Interesting Case of Hyperpigmentation in Two Siblings}

\author{
Deepak Khandelwal, ${ }^{1}$ Sunil Mishra, ${ }^{1}$ \\ Rajesh Khadgawat, ${ }^{1}$ AC Ammini, ${ }^{1}$ \\ and Adrian J.L. Clark ${ }^{2}$
}

${ }^{1}$ Department of Endocrinology and Metabolism,
All India Institute of Medical Sciences, New Delhi
${ }^{2}$ Molecular Endocrinology, John Vane Science Centre,
Barts and the London, London, UK

Adrenal insufficiency in childhood is a rare condition. Familial glucocorticoid deficiency (FGD), also known as hereditary unresponsiveness to ACTH or isolated glucocorticoid deficiency is a rare autosomal recessive disorder that is characterized by severe cortisol deficiency, high plasma ACTH levels and typically, a well-preserved rennin angiotensin-aldosterone axis and hence normal mineralocorticoid levels. FGD is a rare cause of adrenal insufficiency in childhood. We are reporting experience of FGD in two siblings. Our index case, a 3.5 years old male child of Asian-Indian origin presented to the dermatology outdoor with diffuse hyperpigmentation. After ruling out nonendocrinal causes of hyperpigmentation, he was detected to have very high ACTH levels $(3320 \mathrm{pg} / \mathrm{ml})$ and referred to endocrinology. Parents denied any history suggestive of hypoadrenalism, apart from hyperpigmentation. There was no history of fungal infection, alopecia, or tetany. There was no past history of tuberculosis. Genital examination was unequivocally male with both testes descended, without axillary or pubic hair. Endocrine evaluation revealed $8.00 \mathrm{am}$ serum cortisol $5.4 \mathrm{ug} / \mathrm{dl}$ (normal range 6.2-9.4 ug/dl) with plasma ACTH level $3320 \mathrm{pg} / \mathrm{ml}$ (normal range 6-56 pg/ml). Stimulation with synthetic ACTH (Synacthen $250 \mathrm{ug}$ ) failed to increase serum cortisol further $(5.2 \mathrm{ug} / \mathrm{dl})$. Post-exercise and furosemide stimulated serum aldosterone level increased twice the basal values. CT scan of abdomen revealed normal size adrenal glands without any hyperplasia, atrophy or calcification. All possible causes of primary hypoadrenalism were excluded. Family screening showed normal 8 AM serum cortisol $(9.6 \mathrm{ug} / \mathrm{dl})$ with high level of plasma ACTH $(260 \mathrm{pg} / \mathrm{ml})$ in younger sister (aged one year) but without any hyperpigmentation or any other complaint. Test for anti-adrenal antibodies were negative in both siblings and parents. Mutation analysis did not show any mutation in melanocortin-2 receptor (MC2R) or melanocortin receptor accessory protein (MRAP) gene. Both of them are regularly taking treatment (3 years in index case and one and half year in sister) and growing normally. Hyperpigmentation has been markedly decreased. Cases illustrates occurrence of hypoadrenalism in two siblings at very young age with very high ACTH levels and without any detectable mutations.

\section{A Girl with Central Precocious Puberty following Surgical Removal of Virilizing Adrenocortical Tumor}

Tippayakarn Klomchan, Suttipong Wacharasindhu, Vichit Supornsilchai, and Taninee Sahakitrungruang

\author{
Division of Pediatric Endorinology, Department of \\ Pediatrics, Faculty of Medicine, Chulalongkorn University, \\ Bangkok, Thailand
}

Adrenocortical carcinoma, an extremely rare neoplasm in children, may be presented with virilization alone as pseudoprecocious puberty or in combination with glucocorticoid and minerocorticoid excess as cushing syndrome. We report a-2.6-year old girl with adrenocortical carcinoma. She had clitoromegaly, pubic hair, deep voice and accelerated growth for 1 year. Short ACTH stimulation test was reported normal cortisol response and slightly elevation of 17OHP. Serum DHEAS was extremely high. The bone age was advanced to 6.8 years old according to Greulich and Pyle. An abdominal computed tomography revealed a large left adrenal mass. She underwent complete surgical resection and no evidence of metastasis was found. The pathological diagnosis was adrenocortical carcinoma. The virilized symptoms were gradually improved within 1 year and a DHEAS level was rapidly decreased to normal range in 4 months. Her breast developed as Tanner 2 when she was 4 year old. The GnRH stimulation test showed peak FSH of $14.8 \mathrm{IU} / \mathrm{L}$ and $\mathrm{LH}$ $15.3 \mathrm{IU} / \mathrm{L}$. The tumor markers showed AFP $<0.5 \mathrm{IU} / \mathrm{mL}$ and Beta $\mathrm{hCG}<5 \mathrm{mU} / \mathrm{mL}$ and ultrasonography revealed pubertal size and shape of uterus and ovaries. This condition is similar to that is found in some patient with congenital adrenal hyperplasia especially after glucocorticoid replacement. The exact mechanism remained unknown. The gonadotropin releasing hormone agonist was given monthly to delay pubertal progression. In conclusion, an isosexual pubertal sign should be closely monitored after the treatment of virilizing adrenocortical tumor to prevent the progression of puberty especially in girl and attain final adult hight with GnRHa injection.

\section{Analysis the Cardiovascular Risk Factor of Obesity Children with Nonalcoholic Fatty Liver Disease}

\author{
Jiang Li-hong, Liu Ge-li, and Zheng Rong-xiu
}

Tianjin medical university general hospital, pediatric department

Objective. To explore the cardiovascular risk factor of obesity children with nonalcoholic fatty liver disease (NAFLD). Methods. 186 obesity children were divided in to two groups: 114 obesity children with NAFLD (NAFLD group) and 72 was obesity children without NAFLD (WNAFLD group). Body mass index, liver B-mode ultrasound examination, triglycerides (TG), low density lipidprotein-chelesteral (LDL-C), high density lipidprotein-chelesteral (HDL-C) and high-sensitive C-reactive protein (hs-CRP) were performed for all of the cases. Results. (1) The incidence of obesity children with NAFLD was $61.3 \%$ (114/186). 
BMI, HDL-C and LDL-C of NAFLD group were $29.75 \pm$ $3.83,1.16 \pm 0.87 \mathrm{mmol} / \mathrm{L}$, and $2.34 \pm 0.92 \mathrm{mmol} / \mathrm{L}$. BMI, HDL-C and LDL-C of WNAFLD group were $28.53 \pm$ $4.49,1.22 \pm 0.76 \mathrm{mmol} / \mathrm{L}$, and $2.53 \pm 0.81 \mathrm{mmol} / \mathrm{L}$. There were no differences between two groups $(P>.05)$. hsCRP and TG of NAFLD group were $7.84 \pm 2.62 \mathrm{mg} / \mathrm{L}$ and $1.53 \pm 0.85 \mathrm{mmol} / \mathrm{L}$. hs-CRP and TG of WNAFLD group were $3.86 \pm 1.57 \mathrm{mg} / \mathrm{L}$ and $1.28 \pm 0.48 \mathrm{mmol} / \mathrm{L}$. There were significant different between these two groups respectively. Conclusion. The cardiovascular risk factors of obese children are not as same as the obese adults. Obese children with NAFLD are at higher risk of cardiovascular diseases. hs-CRP is one helpful indicator to detect cardiovascular diseases for obesity children with NAFLD.

\section{Prevalence and Risk Factor of Metabolic Syndrome in Childhood-Onset Young Adults with Hypopituitarism}

\section{Han Hyuk Lim, ${ }^{1}$ Min Jae Kang, ${ }^{2}$ Young Ah Lee, ${ }^{2}$ Choong Ho Shin, ${ }^{2}$ and Sei Won Yang ${ }^{2}$}

\author{
${ }^{1}$ Department of pediatrics, School of medicine, \\ Chungnam national university, Daejeon, the South Korea \\ ${ }^{2}$ Department of pediatrics, College of medicine, \\ Seoul national university, Seoul, the South Korea
}

Purpose. This study evaluated the prevalence of metabolic syndrome (MetS) and the risk factors of metabolic derangement for childhood-onset young adults with hypopituitarism (COAHP). Methods. Thirty patients with COAHP with hormone replacement therapy aged 20 to 29 years who visited Seoul National University Children's Hospital between September 2009 and February 2010 were enrolled. Laboratory tests for metabolic syndrome including height, weight, waist circumference (WC), and the hip circumference were performed. The clinical and hormonal features were reviewed, retrospectively. We evaluated metabolic derangement of enrolled patients and healthy adults aged 20' (data from Korean National Health and Nutrition Examination Survey (KNHANES), 2005) by National Cholesterol Education Program-the Adult Treatment Panel III (NCEPATP III). Results. In comparison with KNHANES, the patients with COAHP had more significantly WC size, hypertension, and hypertriglyceridemia. It was significant correlation between the duration of illness and the central obesity $\left(r_{2}=0.546, P=.003\right)$. The prevalence of MetS in COAHP was $10 \%$ (versus 2, 3\% in KNHANES). The incidence of central obesity and prevalence of MetS was higher than KNHANES $(P<.001$ and $P=.042$, resp.). Conclusion. The longer duration of illness not the older age is risk factor for cenral obesity. The insulin resistance caused by central obesity is known key for metabolic syndrome. We should regularly check the WC for preventing MetS, particulary, in COAHP with long history of illness.

\section{Molecular Analysis of the Cyp11b1 Gene}

\section{Loke Kah Yin, ${ }^{1}$ Cindy Ho Wei-Li, ${ }^{2}$ Yvonne Lim Yijuan, ${ }^{2}$ and Poh Kok Seng Larry ${ }^{1}$}

\author{
${ }^{1}$ Department of Paediatrics, \\ National University of Singapore \\ ${ }^{2}$ University Children's Medical Institute, \\ National University Hospital
}

Congenital adrenal hyperplasia is commonly caused by 21 hydroxylase deficiency (90-95\%) and 11 beta-hydroxylase deficiency (5-8\%). Their identical presentation of genital ambiguity (females) and pseudoprecocious puberty (males) can lead to misdiagnosis since hypertension, the clinical hall mark of 11 beta-hydroxylase deficiency, is variable and the biochemical confirmation of elevated precursor metabolites (deoxycortisol/deoxycorticosterone) is not readily available locally. We report the results of molecular characterization of 11 beta-hydroxylase deficiency in 6 patients from unrelated pedigrees (Singapore, Malaysia, India, Nigeria), suspected of having 11 beta-hydroxylase deficiency. All males presented with pseudoprecocious puberty, while the females had virilised external genitalia at birth. Only 1 male presented with hypertension at diagnosis, aged 5 years, and one female had hypertension when she was non-compliant during adolescence. All the other patients did not have hypertension, but had normal CYP21 gene analysis. The CYP11B1 gene was amplified by polymerase chain reaction, and the amplicons were sequenced. Wild-type CYP11B1 gene was amplified from normal human fetal adrenal total cDNA and cloned into the pBUDCE4 mammalian expression vector for the functional expression studies. The DNA could not be amplified in two patients, suggesting a large deletion of the CYP11B1 gene. A total of 4 mutations were discovered in the other 4 patients, including 2 previously described mutations (Q338X and Q356X) and 2 novel mutations (R374W and R454C). The novel mutations were constructed using site-directed mutagenesis, and the constructs were cotransfected with the wild-type CYP21B expression vector into HEK293 cells, prior to the addition of tritium labeled 17-hydroxyprogesterone substrate. After incubation for 24 to 48 hours, there was full conversion of 11-deoxycortisol to cortisol by the wild type 11 beta-hydroxylase enzyme, but there was no conversion with the R374 and R454 mutants, confirming their role in the pathogenicity of 11 beta-hydroxylase deficieny. To our knowledge, this is the first report of novel mutations arising from the Singaporean, Malaysian, Indian and Nigerian population. Molecular diagnosis can provide an alternative confirmation of 11 betahydroxylase deficiency in patients who have a normal CYP21 gene in the absence of hypertension, and would allow for early and effective management of hypertension. 


\section{Congenital Adrenal Hyperplasia-Gender Dysphoria}

\author{
Siska Mayasari Lubis, ${ }^{1}$ Frida Soesanti, ${ }^{2}$ \\ Aman B Pulungan, ${ }^{2}$ Bambang Tridjaja AAP, ${ }^{2}$ \\ and Jose RL Batubara ${ }^{2}$ \\ ${ }^{1}$ Pediatric Endocrinology Division, \\ Child Health Department, Medical School, University of \\ Sumatera Utara, H.Adam Malik Hospital, Medan \\ ${ }^{2}$ Medical School, University of Indonesia, \\ Cipto Mangunkusumo Hospital, Jakarta
}

Background. Congenital adrenal hyperplasia (CAH) is characterized by an impaired biosynthesis of cortisol and aldosterone and an increased secretion of $17 \alpha$ hydroxyprogesterone (17OHP) and androgens. Clinically, almost all forms can be allocated to three forms; the salt wasting (SW), the simple virilizing, and the nonclassic (NC) forms. Patients require long-term glucocorticoid treatment. Late diagnosis may result in death during an SW crisis or in prolonged incorrect gender assignment. Objective: To report a case of late diagnosis of congenital adrenal hyperplasia, simple virilizing form in a 16 years old girl with gender dysphoria. Case: This girl was born with ambiguous genitalia and said to be a girl and further raised as a girl. She grew, developed and act as a girl. There was no intervention for her ambiguous genitalia. Since one year ago she started acting and feel like a boy. She insisted her name change to a boy's name. Because of these reasons, her parents took her to our division to investigate her gender assignment. Physical examination showed a male habitus, body weight: $40 \mathrm{~kg}(<\mathrm{P} 3)$, height: $149 \mathrm{~cm}(<\mathrm{P} 3)$. Her skin looked darker than her parents, with acne, heavy voice, and hirsutism. External genitalia showed clitoromegaly, pubertal stage: A2M1P3 (Tanner scale), and no history of menarche. Analysis chromosome: $46 \mathrm{XX}$, SRY gene: negative. Serum 17 OHP: 302, $60 \mathrm{nmol} / \mathrm{L}$. Pelvic USG: showed uterus and ovarium, there were no right and left testicles, Bone age: average girl. We diagnosed: $\mathrm{CAH}$, simple virilizing form. Unfortunately, the diagnosis was very late and it has revert the patient to be a boy and refused all of the treatments. So, we consulted her to psychiatry department to evaluate the gender identity of this patient. Conclusion. This patient needs more psychoeducation and psychotherapy to make sure her gender identity.

\section{Congenital Adrenal Hyperplasia in Indonesia: A Retrospective Analysis}

\author{
Siska Mayasari Lubis, ${ }^{1}$ Frida Soesanti, ${ }^{2}$ \\ Jose RL Batubara, ${ }^{2}$ Bambang Tridjaja AAP, ${ }^{2}$ \\ and Aman B Pulungan ${ }^{2}$
}

\author{
${ }^{1}$ Pediatric Endocrinology Division, Child Health \\ Department Medical School, University of \\ Sumatera Utara, H.Adam Malik Hospital, Medan
}

\author{
${ }^{2}$ Medical School, University of Indonesia, \\ Cipto Mangunkusumo Hospital, Jakarta
}

Background. Congenital adrenal hyperplasia $(\mathrm{CAH})$ is a recessively inherited disorder in the biosynthesis of adrenal steroids with an incidence of 1 in 5000-18000 newborns of a mixed population. Up two thirds of the cases suffer from the more severe salt-losing form of the disease. This is the first report of CAH cases in Indonesia and there is no neonatal screening program for $\mathrm{CAH}$ in Indonesia. Objective. To review retrospectively the presentation of all known patients with $\mathrm{CAH}$ under the care of pediatricians at Hospitals in Indonesia between 1995 until 2010. Methods. This was a retrospective study, we reviewed all the babies and children who came to Hospitals in Indonesia with diagnosis congenital adrenal hyperplasia that reported by pediatricians. The following information was collected: patient's sex, body mass index (BMI), age at diagnosis, CAH type, parents' ethnic and earning, presenting clinical features, family history, health funding, supporting examinations (17 hydroxyprogesterone (17 OHP), plasma renin activity (PRA), testosterone, bone age, chromosome analysis, genitalia USG), and treatments (Hydrocortisone, Fluorinef, and surgical intervention). Results. One hundred and ten patients with $\mathrm{CAH}$ who were diagnosed between 1995 and 2010 were included in order to be analyzed. In some cases not all the information was available. There were 16 males and 83 females (male:female ratio 1:5), 11 $(10 \%)$ patients were incorrectly assigned sex. The age at diagnosis was between since newborn until 29 years. The $\mathrm{CAH}$ cases were most common happened in Jawa ethnic. Two cases $(1,8 \%)$ had a history of sibling death in the neonatal period, three $(2.7 \%)$ cases similar features were present in older siblings and one patient has $\mathrm{CAH}$ carier parent. Unfortunately, we didnot have data about parental consanguinity. Salt wasting, simple virilization and nonclassical CAH was found in $85(77,3 \%), 21(19,1.0 \%)$ and $4(3,6 \%)$ patients, respectively. Twenty seven $(24,5 \%)$ of them had adrenal crisis, $54(49 \%)$ had ambiguous genitalia, 5 $(4,5 \%)$ had precocious puberty, $1(0,8 \%)$ had amenorrhea, $4(3,6 \%)$ had hypertension, and $10(9 \%)$ had failure to thrive. The parents earning for some patients were about less than 1 million rupiahs, and it had been the big problem not only for patients but also for the doctors, because for some patients they took the medicines irregularly and some parents refused to be checked supporting examinations for $\mathrm{CAH}$, such as $17 \mathrm{OHP}$, chromosome analysis, PRA, because they didnot have enough money, and only about $14(12,7 \%)$ had financial insurance. Sixty seven (60, 9\%) of patients underwent surgical interventions of their external genitalia. Conclusion. Newborns with developmental anomalies of the external genitalia should be diagnosed as early as possible so that medical, psychological, and social complications are minimized. A neonatal screening program for such a disorder can identify infants at risk for the development of life-threatening adrenal crisis and prevent incorrect sex assignment of affected female infants with intersex. 


\section{Autosomal Recessive Pseudohypoaldosteronism Type 1 in a Neonate with 3 Deaths of Siblings}

Palinee Nantarakchaikul, Preamrudee Poomthavorn, and Pat Mahachoklertwattana

\author{
Division of Endocrinology, Department of Pediatrics, \\ Faculty of Medicine, Ramathibodi Hospital, \\ Mahidol University, Bangkok, 10400 Thailand
}

Autosomal recessive pseudohypoaldosteronism type 1 (arPHA1) is a rare genetic disease. It is characterized by renal salt wasting, dehydration, hyperkalemia and metabolic acidosis during the neonatal period. Resistance to aldosterone underlies the clinical presentation. Markedly elevated serum aldosterone level and plasma renin activity (PRA) are thus hallmarks of the disease. Unawareness of the condition makes the diagnosis difficult. We report a female neonate with arPHAl who presented with renal salt wasting at the first week of life. She was born at term with an uneventful pregnancy with a birthweight of $3.5 \mathrm{~kg}$. No history of parental consanguinity was noted. She had 3 earlier born siblings with different sexes died of "suspected" sepsis during the first weeks of lives. Physical examination was unremarkable. Owing to the sibling deaths history, she had been admitted since birth for closed observation and investigations of an inborn error of metabolism. She developed lethargy and poor feeding with dehydration on day 6 of life despite apparently healthy looking and normal serum electrolytes prior. Investigations showed serum electrolytes of $\mathrm{Na}$ $112, \mathrm{~K} 12, \mathrm{Cl} 84$, and $\mathrm{CO} 27.4 \mathrm{mmol} / \mathrm{L}$. Blood urea nitrogen and serum creatinine were elevated at 34 and $0.6 \mathrm{mg} / \mathrm{dL}$, respectively. Plasma glucose level was $105 \mathrm{mg} / \mathrm{dL}$. Septic workup results were all negative. Primary adrenal insufficiency as a cause of hyponatremic, hyperkalemic and metabolic acidosis was suspected. However, serum cortisol level was appropriately raised at $44 \mathrm{mcg} / \mathrm{dL}$. Simultaneous serum aldosterone was markedly elevated at $21,187 \mathrm{pg} / \mathrm{mL}$ (normal, 50-1, 750) and PRA was $10.7 \mathrm{ng} / \mathrm{mL} / \mathrm{h}$ (normal, 1.5-5.7). Pseudohypoaldosteronism was promptly diagnosed. Preliminary genetic study demonstrated a deletion of the gene encoding one of the subunits of epithelial $\mathrm{Na}$ channel (ENac) but no mineralocorticoid receptor gene mutation was found. Therefore, arPHA1 was the most likely diagnosis. She had been treated with large dose of $\mathrm{Na}$ and bicarbonate supplementation with potassium-binding resin since the diagnosis. She has now been followed for 5 years with normal growth and development albeit having frequent episodes of hyponatremic, hyperkalemic and metabolic acidosis during febrile illnesses. This report highlights the importance of suspicion of pseudohypoaldosteronism diagnosis in a neonate with renal salt wasting syndrome. Prompt treatment and long-term follow-up are essential for saving life and having a good outcome.

\section{Pregnancy and Delivery in 3 Women with Congenital Hyperplasia due to 21-Hydroxylase Deficiency}

Satsuki Nishigaki, ${ }^{1}$ Yusuke Mizuno, ${ }^{1}$ Kazuyuki Waki, ${ }^{1}$ Masahiro Noda, ${ }^{1}$ Yasuhiro Naiki, ${ }^{1}$ Toshiaki Tanaka, ${ }^{2}$ Ayako Tanae, ${ }^{3}$ and Reiko Horikawa ${ }^{1}$

\author{
${ }^{1}$ Division of Endocrinology and Metabolism, \\ National Center for Child Health and Development, \\ ${ }^{2}$ Tanaka Growth Clinic, Department of pediatrics, \\ Shonankamakura General Hospital
}

Context. Fertility rate in women with congenital adrenal hyperplasia (CAH) has been reported to be lower than in general population. However, recent studies suggested that the pregnancy outcome of mothers and children would not differ in those with $\mathrm{CAH}$ from general population, once they achieved pregnancies and were treated adequately. We report three cases with 21-hydroxylase deficiency (21OHD) treated at our institution from childhood, who experienced pregnancy and delivery. Cases. Three women with simple virilizing (SV) form $21 \mathrm{OHD}$ conceived and delivered a total of 5 children. Two cases underwent genital reconstruction at age of one and four, respectively. In all three, the menstrual cycles were regular and 17-hydroxyprogesterone (17OHP) levels were controlled within $0.2 \sim 4 \mathrm{ng} / \mathrm{ml}$ before pregnancy. All were able to achieve spontaneous pregnancy. Two cases were treated with dexamethasone before pregnancy, and switched to predonisone after becoming pregnant. One case treated with paramethasone received same medication. During pregnancy, maternal serum 17OHP and testosterone levels were monitored and controlled within normal pregnant range by adjusting the glucocorticoid dosage. The maximum predonisone dose needed was $7 \mathrm{mg}$ /day in one case. The pregnancies were uncomplicated in both mothers and fetuses. Two patients underwent elective Caesarean sections because of prior vaginal reconstruction and expected risk of adrenal failure caused by delivery stress. While, one patient had a vaginal delivery. Stress dose glucocorticoid was given at the time of delivery, and there were no labor complications. All of their babies were born in normal-weight and free from complications and malformations. Among 5 children, three were boys and two were girls. None of the girls had apparent virilization; slightly wide vagino-anal length was observed in one girl. The growth and development have been normal in all children. Discussion. We report three women with SV form $21 \mathrm{OHD}$ who experienced successful pregnancies. The appropriate medical and surgical management from infancy enables spontaneous pregnancy and vaginal delivery. For the management of pregnancy in $21 \mathrm{OHD}$, it is essential to use non-permeable steroids through placenta for prevention of fetal excessive steroid exposure, and the adjustment of steroid dosage by monitoring maternal serum 17OHP and testosterone levels for prevention of virilization in female fetus. The optimizing dosage of corticosteroid should be individualized. The improvement of pregnancy rate in saltwasting 21OHD remains an issue to be solved. 


\section{Carrier Frequency of Congenital Adrenal Hyperplasia (21-hydroxylase deficiency) in Korean Children with the Majority of Early Puberties}

\author{
Phil Soo Oh, ${ }^{1}$ Hui Kwon Kim, ${ }^{1}$ Hong Jin Lee, ${ }^{1}$ \\ and Jae Hoon Shin² \\ ${ }^{1}$ Pediatrics, Hallym University Medical Center, \\ Chuncheon, Korea \\ 2 Pediatrics, Hanyang University Medical Center, \\ Seoul, Korea
}

Objective. Nowadays, many Koreans are concerned with the phenomenon of early puberty. And I know a few reports of congenital adrenal hyperplasia $(\mathrm{CAH})$ carrier frequency in some populations. So, prospectively I scheduled to know the CAH (21-hydroxylase deficiency) carrier frequency in Korean children with the majority of early puberties. Methods. I started to gather the study group since July, 2008. So far, ACTH stimulation tests have been done for 171 Korean children (female 161, male 10) with the majority of early puberties and the results of 17-OH-progesterone (17OHP) and cortisol have been analyzed with bone age evaluation etc. Results. I found about 10 heterozygote CAH carriers in the study group. And I could find an interesting aspect of $17 \mathrm{OHP}$ results. Almost always the cortisol responses after ACTH stimulation were elevated, but the $17 \mathrm{OHP}$ responses were not. In many children, $17 \mathrm{OHP}$ were not sufficiently elevated, or even the same or decreased responses could be seen among them. Conclusions. I think that the heterozygote CAH (21hydroxylase deficiency) carrier frequency would be about $5 \%$ in Korean children with the majority of early puberties. And it seems that another mechanism(s)/pathway(s) of ACTH stimulation for cortisol could be present.

\section{An Unusual Case of Hypoglycaemic Seizure in a Male Term Chinese Neonate}

Grace WK Poon, PT Cheung, KW Chan, Joanna YL Tung, Anne MK Kwok, and Louis CK Low

\section{Department of Paediatrics and Adolescent Medicine, Queen Mary Hospital, The University of Hong Kong}

Introduction. X-linked adrenal hypoplasia congenita (Xlinked AHC) is a rare cause of adrenal insufficiency in male infants presenting in the neonatal period. It results from a deletion or point mutation of the NR0B1 (nuclear receptor subfamily 0 , group $B$, member 1 ) gene which encodes an orphan nuclear receptor DAX1 (dosage-sensitive sex reversal-adrenal hypoplasia congenita critical region on the $\mathrm{X}$ chromosome, gene 1) that is expressed in the hypothalamus, pituitary gland, adrenal gland and gonads. Case Report. A normal-sized male term infant initially developed cyanosis and seizure on day 2 of life. He was found to have hypoglycaemia and high $\mathrm{C}$-reactive protein and was treated as clinical sepsis. The plasma electrolytes, CXR, echocardiogram and USG brain were all normal and sepsis screening including blood and cerebrospinal fluid were negative for bacterial culture. He was discharged after one week of intravenous antibiotics but was readmitted to hospital 10 days later with shock and acute adrenal insufficiency, characterized by severe hyponatraemia, hyperkalaemia and metabolic acidosis. He required fluid resuscitation as well as full ventilatory and inotropic support. Clinically he had no hyperpigmentation. The serum cortisol level was low $(132 \mathrm{nmol} / \mathrm{L})$ at the time of stress; together with a normal plasma 17-hydroxyprogesterone $(12 \mathrm{nmol} / \mathrm{L})$ and normal USG adrenals, 21-hydroxylase deficiency was excluded. His normal male external genitalia excluded 3b-hydroxysteroid dehydrogenase deficiency or a deficiency of steroidogenic acute regulatory protein (StAR). His plasma renin activity was high $(>50 \mathrm{ng} / \mathrm{ml} / \mathrm{hr})$ whilst the aldosterone level was normal $(255 \mathrm{pmol} / \mathrm{L})$, suggesting a biosynthetic defect of aldosterone. The clinical picture was compatible with congenital adrenal hypoplasia and the baby recovered well with hydrocortisone, mineralocorticoid replacement and salt supplement. Genetic analysis: PCR-based cycle sequencing of the 2 exons of the NR0B1 gene was performed on peripheral blood leucocyte-derived DNA from the proband. The baby was found to be hemizygous for a known mutation c.1292delG (TAGTA>TATA), leading to frameshifting and a premature stop codon (p.S431fs436X) 1. Conclusion. Despite its rarity, it is essential for paediatricians to maintain vigilance over the possible diagnosis of $\mathrm{X}$-linked $\mathrm{AHC}$ in male infants presenting with severe adrenal insufficiency in the newborn period. Affected male may also present later in childhood or adolescence with delayed puberty from hypogonadotropic hypogonadism. Female carriers do not develop adrenal insufficiency but may present with delayed puberty. Genetic analysis may aid the anticipation of clinical problem in such patients; it may also be valuable in carrier identification and prenatal counselling.

\section{Cushing Syndrome in King Culalongkorn Memorial Hospital: A Case Series}

\section{Rottanat Rugpolmuang \\ Endocrine Unit, Department Of Pediatrics, Chulalongkorn University}

Endogenous Cushing syndrome in childhood and adolescence is rare. Six children, 2 girls and 4 boys, were diagnosed as having Cushing syndrome and admitted to King Chulalongkorn Memorial Hospital between 2006 and 2010. Four of 6 were diagnosed as Cushing disease and 2 of 6 as adrenocortical carcinoma. The clinical presentations, investigations, management and therapeutic outcome were retrospectively reviewed. The clinical data were shown in the table. In addition to the typical clinical features of cushing syndrome, patient with adrenocortical carcinoma always present with heterosexual precocious puberty. The age of onset of adrenocortical carcinoma group were younger (6-12 years) than that of cushing disease (12-14 years). Loss of circardian rhythm and cortisol unsuppressible from LDDST (Low dose dexamethasone suppression test) were 
demonstrated in all patients. One of 4 patients with cushing disease can not be suppressed by the HDDST (high dose dexamethasone suppression test). One of 4 can not be seen by the Pituitary imaging but the tumor was localized by the bilateral inferior petrosal sinus sampling (BIPSS). One patient with Pituitary macroadenoma has a consequences as diabetes insipidus and the others were normal after surgical resection. All the patients with adrenocortical carcinoma were treated by surgical removal and chemotherapy. No evidence of metastasis were found in the patients with adrenocortical carcinoma, but one patient needed to be reoperation due to the tumor recurrence. There were no clinical overt signs of adrenal insufficiency due to all of them were replaced with glucocorticoid and gradually decreased to physiologic requirement for at least 6-9 months after treatment.

\section{Partial Defect in the Cholesterol Side-Chain Cleavage Enzyme P450scc (CYP11A1) Presenting as Non-Classic Congenital Lipoid Adrenal Hyperplasia}

\author{
Taninee Sahakitrungruang, 1,2 Piers R. Blackett, ${ }^{3}$ \\ and Walter L. Miller ${ }^{1}$ \\ ${ }^{1}$ Department of Pediatrics (TS, WLM), University of \\ California, San Francisco, San Francisco, CA 94143; \\ ${ }^{2}$ Departments of Pediatrics, Faculty of Medicine, \\ Chulalongkorn University, Bangkok, Thailand (TS); \\ ${ }^{3}$ Departments of Pediatrics (PRB), University of \\ Oklahoma Health Sciences Center, Oklahoma City, \\ OK 73104
}

Context. The cholesterol side-chain cleavage enzyme (P450scc), encoded by the CYP11A1 gene, converts cholesterol to pregnenolone to initiate steroidogenesis. Genetic defects in $\mathrm{P} 450$ scc cause a rare autosomal recessive disorder that is clinically indistinguishable from congenital lipoid adrenal hyperplasia (lipoid CAH). Non-classic lipoid $\mathrm{CAH}$ is a recently recognized disorder caused by mutations in the steroidogenic acute regulatory protein (StAR) that retain partial function. Objective. We describe two siblings who appeared to have non-classic lipoid $\mathrm{CAH}$ but had P450scc mutations that retain partial function. Patients and Methods. A 46, XY male presented with underdeveloped genitalia and partial adrenal insufficiency; his 46, XX sister presented with adrenal insufficiency. Hormonal studies suggested partial primary adrenal and gonadal insufficiency. Sequencing of the StAR gene was normal, but mutations were found in the CYP11A1 gene. Mutations were recreated in the F2 plasmid expressing a fusion protein of the cholesterol side chain cleavage system. P450scc activity was measured as pregnenolone production in transfected COS-1 cells. Results. The patients were compound heterozygous for the previously-described frameshift mutation 835delA ( $0 \%$ activity) and the novel missense mutation A269V, which retained $18.1 \%$ activity of the wild-type F2 protein. Conclusions. There is a broad clinical spectrum of P450scc deficiency. Partial loss-of-function CYP11A1 mutation can present with clinical and hormonal phenotypes indistinguishable from non-classic lipoid CAH.

\section{A Longitudinal Study on Adrenarche Progress in 49 Girls with Idiopathic Central Precocious Puberty (Icpp) and Elevated Dehydroepiandrosterone Sulfate (Dheas) before and during Gnrh Analogue Treatment}

\section{Zhe Su}

\section{The First Affiliated Hospital of Sun Yat-Sen University, Guangzhou, $P R$ China}

Objectives. To explore the relationship between andrenarche and gonadarche. Methods. One hundred and sixty one girls with ICPP were evaluated. We followed up 49 of them whose serums DHEAS Z score for chronological age (CA) were higher than $+2 \mathrm{SD}$ at diagnosis. Physical examinations including pubertal stage were repeated at 3-6-month interval. Serum DHEAS levels were monitored yearly. They were followed up for an average period of 3.26 years after $\mathrm{GnRH}$ analogue $(\mathrm{GnRHa})$ treatment. We were able to follow up 16 patients until more than one year after discontinuation of GnRHa treatment. Results. All the 49 girls with ICPP and elevated serum DHEAS were older than 6 years old. Before GnRHa treatment, the 49 girls presented younger average age at attainment of pubic hair stage 2 ( $\mathrm{PH} 2)$ and pubic hair stage $3(\mathrm{PH} 3)$ than normal $8.07 \pm 0.98$ years versus $11.16 \pm 1.15$ years and $8.82 \pm 0.51$ years versus $12.40 \pm 1.28$ years, respectively, $(P<.01)$. During GnRHa treatment, the intervals between $\mathrm{PH} 2$ and $\mathrm{PH} 3, \mathrm{PH} 3$ and pubic hair stage 4 (PH4), breast stage 2 (B2) and $\mathrm{PH} 2$ were longer than normal $1.69 \pm 0.67$ years versus $0.83 \pm 0.52$ years, $1.64 \pm 1.07$ years versus $0.60 \pm 0.30$ years and $3.62 \pm 1.40$ years versus $0.76 \pm 0.99$ years, respectively, $(P<.01)$. The intervals between $\mathrm{PH} 2$ and $\mathrm{PH} 3, \mathrm{~B} 2$ and $\mathrm{PH} 2$ during GnRHa treatment were also longer than that before GnRHa treatment $1.69 \pm 0.67$ years versus $1.05 \pm 0.33$ years, $3.62 \pm 1.40$ years versus $1.13 \pm 0.91$ years, respectively, $(P<.01)$. The $\triangle$ DHEAS SDS per year during GnRHa treatment were $(-0.57 \pm 1.12, n=49)$, $(-0.61 \pm 0.88, n=49),(-0.75 \pm 0.95, n=24),(-0.84 \pm$ $0.89, n=7)$ respectively. It increased significantly after GnRHa cessation, $(0.78 \pm 1.64 n=16)$. Conclusion. It was observed that gonadarche after 6 year old may lead to earlier adrenarche. GnRHa treatment may slow down adrenarche progress except for suppressing the hypothalamus-pituitarygonadal axis.

\section{Expressions of SEDL Gene and Its Encoded Protein Sedlin in a Variety of Tissues in Mice}

Feng Xion, $\mathrm{Na} \mathrm{Hu}$, and Fei Lao

Children's Hospital of Chongqing Medical University

Objective. To investigate the expressions of SEDL gene and its encoded protein sedlin in a variety of tissues in mice. 
Methods. Real-Time PCR and Western Blot were used to detect the expression levels of the SEDL mRNA and sedlin protein in 9 tissues in mice, respectively. And the expression levels of them in bone tissues in mice of 1,2 and 4-week-old were also measured in the same way. Results. (1) Expressions of SEDL gene and its corresponding sedlin protein existed in 9 tissues detected in mice. The relative expression levels of SEDL mRNA and sedlin protein in tissues in mice were significantly different $(P<.0001)$, and with a high level in bone. (2) In bone tissues of age varied mice, the relative expression levels of SEDL gene and sedlin were obviously different $(P<.01)$, and age-related changes in expressions were identical with each other. Conclusion. The expression of SEDL gene and sedlin were consistent in tested tissues. Expression levels of them were different among tissues and with a high level in bone. In bone tissues of mice, the expressions of SEDL gene and sedlin had the same changes with time.

\section{A Study on the Contents of Calcium, Magnesium in Northeast Children's Hair}

\section{Guo Xuwei, Zhang yining, Li Yanchun, and Li Shanyu}

\section{The First Affiliated Hospital, Jilin University}

Objective. Calcium is the most occupied inorganic elements in human body and it is one of the essential nutrients and closely related with the activities of human life and disease. Metabolism of calcium and magnesium should not be separated. Calcium involves in several of physiological functions, such as nerve excitability, muscle contraction, heart activity and blood clotting regardless of its small volume in a body. Calcium is an essential material for life activities since it participates in the whole activities of human life. The aim of this study is to determine the levels of calcium, magnesium in northeast children's hair and the result may help us to make more rational nutritional guide for children. Method. In Jilin Province, the volume of calcium and magnesium in hairs are determined by plasma generator from 9158 healthy children that are between three and seven years old. Results. Data shows that the contents of calcium and magnesium in the hair of mostly children in Jilin Province are low than normal level. $77.5 \%$ of the children is lack of calcium whereas $26.2 \%$ of the children shows magnesium deficiency. Calcium deficiency is parallel with magnesium deficiency. Conclusion. in Jilin province, the long winter, lack of sunlight exposure and low calcium and magnesium in food attribute to sub-clinical state of many children. Children in Jilin province need much more outdoor activities and increase calcium intake (at least intake recommended daily dosage) even for those lack of clinical manifestations.

\section{Pituitary Disorders}

\section{A Large Deletion of Prop1 Gene in Patients with Combined Pituitary Hormone Deficiency from Two Unrelated Chinese}

\author{
Zhang Huiwen, ${ }^{1}$ Wang $\mathrm{Yi}^{1}{ }^{1}$ Han Lianshu, ${ }^{1}$ \\ Gu Xuefan, ${ }^{1}$ and Shi Dingping ${ }^{2}$ \\ ${ }^{1}$ Xinhua Hospital, Shanghai Institute For \\ Pediatric Research, Shanghai Jiaotong University School \\ Of Medicine \\ ${ }^{2}$ Beijing Children Growth Expert Clinic
}

Background. Combined pituitary hormone deficiency (CPHD) has an estimated prevalence of $1: 8000$. Familial CPHD is more likely to have a genetic cause than sporadic CPHD. Mutations in PROP1 gene encoding pituitaryspecific transcriptional factor is the most common cause for CPHD. Herein, we investigated whether PROP1 plays a role in two Chinese familial cases of CPHD. Methods. PROP1 gene and adjacent $5^{\prime}$ and $3^{\prime}$ boundaries from genomic samples from two unrelated families were amplified by polymerase chain reactions (PCR), and directly sequenced to investigate molecular variations and define the extension of potential deletion. Quantitative real-time (RT-PCR) were also conducted to analyze the copy-number of PROP1 gene in the mother of proband. The relationship of the two distantly located families was further analyzed using micorsatellite markers flanking the deleted region. Results. A segment of $\sim 53.2 \mathrm{~Kb}$, comprehending PROP1 and another gene encoding a hypothetical protein Q6ZTH3, was deleted in both pedigrees families. The mother of one of the probands was hemizygote for this large deletion by RT-PCR, which confirmed to the assumption that the affected children inherited each of the deletion allele from their consanguineous parents. The differences of most microsatellites surrounding the absent segment indicated two pedigrees in our study were genetically unrelated. Conclusion. We report the largest genomic deletion including PROP1 gene associated with CPHD. The hypothetical protein Q6ZTH3 is unlikely exert an indispensable function during embryogenesis or organogenesis. The segment $7.7 \mathrm{~Kb}$ upstream to the transcription of PROP1 probably harbors a fragile-site for occurrence of breakpoints, although, the definite site of breaking is still elusive.

\section{Case Series on Pediatric Prolactinoma}

\section{Joanna Tung, Grace Poon, Anne Kwok, Pik To Cheung, and Louis Low}

\section{Department of Paediatrics and Adolescent Medicine, Queen Mary Hospital, The University of Hong Kong}

Introduction. Prolactinomas are the most common hormone-secreting pituitary tumors and they have been reported in patients from the age of 2 to 80 . Although they are uncommon in children and adolescent, they represent $50 \%$ of all pituitary adenomas and $2 \%$ of all intracranial 
tumors. Methods. We retrospectively reviewed the clinical presentation, response to medical treatment and long term outcome of 5 Chinese adolescents ( 4 female, 1 male) with prolactinoma, diagnosed at the age of 12-16 years. Results. The mean duration of follow up was 5 years (2 to 8 years). Only 2 of the 5 patients presented with neurological symptoms including headache and visual disturbance. 3 of the 4 female patients were post-menarcheal and they presented with secondary amenorrhea. 2 out of these 3 girls had galactorrhoea. Growth arrest was not observed in any of the patients and pubertal development was normal in all patients. All patients had macroprolactinoma and the mean size was $19.8 \mathrm{~mm}$ (range: $10-39 \mathrm{~mm}$ ) at presentation while the mean serum prolactin at diagnosis was $41660 \mathrm{miu} / \mathrm{L}$ (range: 1843-179070 miu/L). Only one patient had impairment of other pituitary hormones on presentation. One patient received transphenoidal surgical resection of the prolactinoma before medical treatment and she developed growth hormones deficiency post-operatively. All patients were treated with bromocriptine at doses ranging from $2.5 \mathrm{mg}$ to $15 \mathrm{mg}$ per day. All patients achieved normal serum levels of prolactin but 2 patients were subsequently switched to carbergoline because of intolerable side effects of bromocriptine including postural dizziness and nausea. Carbergoline was well tolerated and normal serum levels of prolactin were achieved at a dose of 0.75 to $1.75 \mathrm{mg}$ per week orally. After 2-8 years of treatment, 2 patients achieved complete resolution of tumor on magnetic resonance imaging (MRI). Another 2 patients showed significant reduction of the tumor size, whilst the remaining one patient showed no interval change. Conclusion. Medical treatment with dopamine agonist is safe and effective in paediatric patients with prolactinoma and should be considered as the first line of therapy.

\section{Endocrine Tumors}

\section{Two Cases of Gigantism Caused by Growth Hormone Secreting Pituitary Adenoma}

\section{Chan Jong Kim, Hwa Jin Cho, and Young Jong Woo \\ Department of Pediatrics, Chonnam National University Medical School \& Hospital, Gwangju, South Korea}

Introduction. Gigantism indicates growth hormone $(\mathrm{GH})$ excess that occurs during childhood when open epiphyseal growth plates allow for excessive linear growth. Gigantism may begin at any age prior to epiphyseal fusion. The incidence of gigantism is extremely rare with approximately 100 reported cases to date. Cases. Case one was a 15-year-old boy who came to our hospital for his tall stature and growth acceleration. On physical examination, all growth parameters were affected: his height and weight were $198.4 \mathrm{~cm}$ (>97 percentile), and $84.2 \mathrm{~kg}$ (>97 percentile), respectively, and his head circumference was $58.5 \mathrm{~cm}$ (>97 percentile). He has been noted to have disproportionately large hands and feet, but there was no evidence of frontal bossing, prominent jaw, or other coarse facial features. On admission, he had done hormonal studies which showed GH $22.7 \mathrm{ng} / \mathrm{mL}$, IGF-1 (somatomedin-C) $740.0 \mathrm{ng} / \mathrm{mL}$, and IGFBP-3 $6301.6 \mathrm{ng} / \mathrm{mL}$, and his $\mathrm{GH}$ was not suppressed on $75 \mathrm{~g}$ oral glucose tolerance test (OGTT). Brain magnetic resonance imaging (MRI) showed $12 \mathrm{~mm}$ macroadenoma in pituitary gland. The tumor was totally removed by transsphenoidal approach, and microscopic findings revealed the tumor turned out to be growth hormone secreting type of pituitary adenoma. After the surgery, GH level was $9.4 \mathrm{ng} / \mathrm{mL}$, and $75 \mathrm{~g}$ OGTT showed suppressed growth hormones. Case two was a 14-year-old boy complained tall stature. His height and weight were $197.3 \mathrm{~cm}$ (>97 percentile) and $101.5 \mathrm{~kg}$ (>97 percentile), respectively, and his head circumference was $58.6 \mathrm{~cm}(>97$ percentile). Endocrine evaluation revealed $\mathrm{GH} 7.1 \mathrm{ng} / \mathrm{mL}$, IGF-1 $518.0 \mathrm{ng} / \mathrm{mL}$, and IGFBP-3 $3450.0 \mathrm{ng} / \mathrm{mL}$, and $\mathrm{GH}$ was not suppressed on $75 \mathrm{~g}$ OGTT (peak GH $18.8 \mathrm{ng} / \mathrm{mL}$ ). Brain MRI showed $6 \mathrm{~mm}$ microadenoma in left side pituitary gland. The tumor was totally removed, and microscopic findings showed growth hormone secreting type of pituitary adenoma. After the surgery, GH was $0.2 \mathrm{ng} / \mathrm{ml}$, and $75 \mathrm{~g}$ OGTT showed suppressed growth hormones. Conclusion. The authors report two cases of gigantism which were due to the growth hormone secreting type of pituitary adenoma requiring transsphenoidal surgery.

\section{Insulinoma Presenting as Hypersomnolence}

\section{Catherine Anne G. Pangilinan}

\section{University of the Philipppines-Philippine General Hospital}

Insulinomas manifest with neuroglycopenic symptoms induced by hypoglycemia. Most cases often initially present with neuropsychiatric symptoms and are often misdiagnosed. This is a case of an 18-year-old girl who presented with 5-year history of increased sleeping time (at least 14 hours/day) associated with difficulty waking up. The patient was initially managed by a psychiatrist as a case of Primary Hypersomnolence after Cranial CT scan, and EEG showed normal results. She was given mirtazapine for one month which was later shifted to fluoxetine with no improvement of symptoms. After three years, she started to develop occasional episodes of generalized tonic-clonic seizures lasting for 10-15 seconds, occurring at least two to three times a month. A repeat EEG showed focal slowing over the left frontal, temporal, and parietal regions suggesting cortical dysfunction but with no epileptiform discharges. Cranial MRI done was unremarkable. The patient was first seen by the endocrinology service during an ER admission for seizure. At that time, the patient was afebrile, with stable vital signs but was noted to hypoglycemic (CBG: $<20 \mathrm{mg} / \mathrm{dl})$. She was drowsy and disoriented postictally which improved upon administration of D50 water. On further evaluation, patient was observed to have a regressed, slow-mannered speech. She also had poor memory and concentration. Hormonal workup of critical samples obtained revealed an elevated insulin level (62.5, NV: 5-35 uiu/mL with simultaneous RBS $5.2 \mathrm{mg} / \mathrm{dL})$, elevated cortisol (820, NV: $160-620 \mathrm{nmol} / \mathrm{L})$, and elevated Growth Hormone (70.9, NV: 0-14 uiu/ml). 
Urine ketone was negative. Holoabdominal ultrasound was normal. Abdominal CT Scan revealed a fatty liver with a mildly prominent pancreatic body; cannot rule out an underlying mass lesion. MRI of the Abdomen showed a welldefined tumor mass in the pancreatic body measuring $2.9 \times$ $3.1 \times 2.6 \mathrm{~cm}$, to consider an islet cell tumor. CBG monitoring in the ward revealed hypoglycemic episodes. Cornstarch was supplemented to diet. The patient subsequently underwent distal pancreatectomy with splenectomy. Intraoperatively, a $2.0 \times 3.0 \times 3.0 \mathrm{~cm}$ mass at the body of the pancreas was identified and excised. Histopathologic findings were consistent with insulinoma. CBG postop ranged between 147-260 mg/dL. Human Regular Insulin was given but was discontinued on the 6th postop day with normalization of blood glucose levels. The patient had improved well-being after the surgery. There was resolution of the hypersomnolence and had no recurrence of seizures. She was also referred to a speech therapist for improvement of speech.

\section{Diagnostic Value of Serum Levels of $\beta$-Human Chorionic Gonadotropin ( $\beta$-hcG) Combined with $\beta$-hcG in Cerebrospinal Fluid for Determination of Locations of Germinomas in Children with Precocious Puberty}

\section{YanHong Li, Zhe Su, HuaMei Ma, HongShan Chen, YuFen Gu, and MinLian Du}

\section{The First Affiliated Hospital of Sun Yat-Sen University, GuangZhou, China, 510080}

Objective. To study the clinical manifestations of germinoma in children with precocious puberty and to evaluate the diagnostic value of serum levels of $\beta$-human chorionic gonadotropin ( $\beta$-hcG) combined with detections of $\beta$-hcG in cerebrospinal fluid (CSF). Method. 12 male children with germinomas confirmed by pathology from Jan, 2005 to Dec, 2009 , in the first affiliated hospital of Sun Yat-Sen University, Guangzhou, aged from 4.15 to 10.18 years, were enrolled in our study. Patients were classified into two groups according to tumor locations: intracranial group and nonintracranial group. Levels of $\beta$-hcG in serum as well as in CSF were detected before the initiation of therapy. Age and gender matched 5 children undergoing lumbar puncture for other diseases who were set as control group of the determinations of $\beta$-hcG in CSF. Levels of $\beta$-hcG and testosterone in serum and CSF were compared between intracranial group and nonintracranial group, and levels of $\beta$-hcG in CSF were compared between nonintracranial group and control group. Results. 12 children showed low response to LHRH stimulation tests but elevated serum levels of testosterone: $29.49 \pm 70.79 \mathrm{ng} / \mathrm{ml}$. The first clinical manifestation in children with non-intracranial or pineal geminomas was precocious puberty, while it was hemiparesis in children with basal ganglia germinomas. Serum levels of $\beta$-hcG were elevated in both intracranial and non-intracranial group, and no differences were found between groups $113.41 \pm$ $112.6551 .96 \pm 24.12(P=.644)$. No relations were found between serum levels of $\beta$-hcG and ages, tumor locations, courses of the patients $r=0.366, P=.24, r=-0.276$, $(P=.384), r=-0.323,(P=.306)$. Levels of $\beta$-hcG in CSF were significantly higher in intracranial group than that in non-intracranial group $355.70 \pm 345.98,1.30 \pm 0.17$ $(P=.009)$. Children with nonintracranial germinomas had similar levels of $\beta$-hcG in CSF as that in control group $1.30 \pm 0.17,1.20 \pm 0.01(P=.571)$. Conclusion. Detection of serum levels of $\beta$-hcG combined with $\beta$-hcG levels in CSF is useful for determination of the locations of germinomas in children with precocious puberty.

\section{The Etiology and Clinical Characteristics of Diabetes Insipidus in Children}

\author{
XiaoJing Liu, HuaMei Ma, Zhe Su, MeiNa Liu, \\ and YanHong Li
}

\section{The First Affiliated Hospital of Sun Yat-Sen University, GuangZhou, China, 510080}

Objective. To study the etiology and clinical features of diabetes insipidus (DI) in children. Methods. From 1997 to 2009, 52 children (44 boys, 8 girls, aged 7.9 years) were confirmed as DI in the first affiliated hospital, Sun YatSen University. Clinical data was learned retrospectively. Results. In our whole group, 7 (13.5\%) were confirmed as nephrogenic diabetes insipidus (NDI), while $45(86.5 \%)$ were confirmed as central diabetes insipidus (CDI). In children with CDI, $29(64.4 \%, 29 / 45)$ were idiopathic, 16 $(35.6 \%, 16 / 45)$ were organic, secondary to brain tumors (11 cases), autoimmune diseases ( 1 case), and central nervous system leukemia (1 case). Short stature was more common in children with idiopathic CDI than that in children with organic CDI (15/29 versus 3/16). 35 children with CDI underwent head MRI scans. Conclusion. Idiopathic CDI still accounts for a large proportion in DI in children. Brain tumors are the leading cause of organic DI. MRI scans of sella region can help to achieve a correct diagnosis at first visit.

\section{Disorders of Sex Development}

\section{Glucocorticoid Therapy for Girls with Cogenital Adrenal Hyperplasia: Effect on Appearance of External Genitalia}

\section{Ariachery Ammini and Rajeev Khanna}

\section{AlIMS, New Delhi}

Girls with congenital adrenal hyperplasia are born with malformed external genitalia. Antenatal glucocorticoid therapy does decrease the extent of malformation. We have observed that postnatal steroid therapy, started during early infancy, can also bring about growth and development of female external genitalia. Presented here is a case to illustrate the morphogenesis of genitalia with medical treatment. Case Report. Baby L S was assigned male sex at birth although urethral opening was at the base of phallus. During 
the second week of life, he was hospitalized because of vomiting. Investigations done during this admission revealed that the karyotype was 46, XX, and the child had salt wasting form of congenital adrenal hyperplasia. Treatment was started with hydrocortisone and fludrocortisone, and parents were advised to rear the child as a female. Photograph to show appearance of genitalia at 1, 6, and 9 months after initiating therapy will be presented to document genital development with medical therapy alone. Discussion. We had speculated, based on observations on human fetuses, that feminization of external genitalia took place during the 3rd trimester of gestation under the influence of estrogen from fetal ovaries. Genital malformations observed in girls with ambiguous genitalia are due to lack of progression of feminization due to inadequate fetal ovarian functions and continuing androgen excess. Subsequently, studies in girls with aromatase deficiency and estrogen receptor defects have also suggested role of estrogen in female genital development. The present study documents postnatal growth and development of external genitalia with steroid supplementation. This has important implications for timing of girls born with genital ambiguity.

\section{Endocrinological Problems in Turner's Syndrome}

\section{Rui Min Chen, Xiang Quan Lin, and Xiao Hong Yang}

\section{Fuzhou Children's Hospital Of Fujian}

Objectives. Evaluating the endocrinological problems in Turner's syndrome (TS). Methods. 49 patients with TS diagnosed in our clinic in 1998 2009 by karyotype, growth hormone stimulation, IGF1, TPO and Tg antibodies, sTSH, FT3, FT4, fasting glucose (GS), Alc (if GS high), insulin, $\mathrm{FSH}, \mathrm{LH}$, ultrasound (ovaries, uterus, and thyroid), bone age, and pituitary MRI (if growth hormone deficiency). Results. Chronological mean age: 11.5 (3.6 16.8) years, height $-3.9(-0.39 \sim-6.25) \mathrm{SD}$, bone age $9.5(2 \sim 13.6)$ years, $>13.5$ years no puberty signs $(13 / 15,87 \%)$, obesity $(6 / 49,12.2 \%)$, and overweight $(6 / 49,12.2 \%)$. Distribution of karyotype: X monosomy -45, X (21/49, 42.9\%), mosaicism (16/49, 32.7\%): including 45X/46, XX, 45, X/46, X, +mar, 45XO/47, XXX, 45X/46, XX/47, XXX, 45X/46X, dufX) (q24), 45X/46X, i(X), 45X/X, idic(X) (q24), 45X/46, XY, $45 X / X, \operatorname{idic}(X)(q 24)$; aberration of $X$ structure (10/49, 20.4\%): 46X, i (X), 46X, $\operatorname{del}(\mathrm{X})$ (q22), and others. Growth hormone deficiency $(25 / 47,53.2 \%)$, IGF1 (207 \pm 130$) \mathrm{ng} / \mathrm{ml}$, Hashimoto thyroiditis $(6 / 26,23.1 \%)$, hyperthyroidism $(2 / 47$, $4.3 \%)$, hypothyroidism $(2 / 47,4.3 \%)$, diabetes $(1 / 49,2 \%)$, and insulin $(12.6 \pm 5.7) \mathrm{IU} / \mathrm{L}, \mathrm{LH}(13.2 \pm 11.1) \mathrm{IU} / \mathrm{L}$, FSH $(77.9 \pm 51.5)$ IU/L. Pituitary MRI abnormalities (5/18, $27.8 \%)$ : including small $(2 / 18,11.1 \%)$, small cyst $(1 / 18$, $5.6 \%)$, and empty sella turcica $(1 / 18,5.6 \%)$, and pituitary tumor or pituitary hyperplasia $(1 / 18,5.6 \%)$ ? Conclusions. Endocrinological problems are common in TS. Patients with TS are at risk for some endocrinological problems that require care early.

\section{A Novel MicroRNA Targeting Gas6 Regulates Vascular Smooth Muscle Cells Apoptosis in Mice and Contributes to Artery Calcification in Children with Chronic Kidney Disease}

\section{Rong-Rong Cui and Ding-An Mao

\begin{abstract}
Pediatric Department, The Second Xiangya Hospital Of Central South University
\end{abstract}

Vascular smooth muscle cells (VSMCs) apoptosis plays a key role in artery calcification, while arterial medial calcification is a major complication in children with chronic kidney disease (CKD) and a strong predictor of cardiovascular and all-cause mortality. MicroRNAs (miRNAs) interfere with translation of specific target mRNAs and are thought to thereby regulate many cellular processes. Recent studies have suggested that miRNAs might play a role in VSMCs apoptosis and artery calcification. Here, we identify a new miRNA (miR-X) in primary mouse VSMCs that promotes VSMCs apoptosis by repressing Gas6 expression at the posttranscriptional level. Overexpression of miR-X enhanced the apoptosis of mouse VSMCs whereas inhibition of miR-X expression attenuated it. Gas6, an inhibitor of cell apoptosis, was confirmed to be a target of miR-X. In calcificationprone DBA/2 mice, CKD was induced by renal ablation of varying magnitudes plus phosphate feeding. In vivo silencing of miR-X in calcification-prone DBA/2 mice enhanced Gas6 expression, inhibited VSMCs apoptosis, and inhibited artery calcification. Importantly, miR-X was found to be conserved in humans, and a homozygous mutation in premiR-X that caused over expression of miR-X was shown to cause artery calcification in 2 related children. Consistent with the mouse data, Gas6 levels were inhibited in the 2 affected individuals. Thus, our studies show that miR-X plays an important physiological role in VSMCs apoptosis and contributes to artery calcification in children with chronic kidney disease.

\section{WT1 Mutation as a Cause of 46XY DSD and Wilm's Tumor: A Case Report and Literature Review}

\author{
Jun Fen Fu, Yang Li Dai, Fang Hong, Shan Xu, \\ and Zheng Shen
}

\section{The Children's Hospital Of Zhejiang University School Of Medicine}

The Wilms' Tumor gene is thought to have tumor suppressor activity and to play an important role in the development and subsequent normal function of the urogenital system. WT1 mutations will impair Gonadal and urinary tract development and have been demonstrated to cause syndromes of WAGR, Denys-Drash, and Fraiser. Here, we describe a 14-year-9-month nonmosaic XY sex-reversed female with pure gonadal dysgenesis (46, XY karyotype, completely female external genitalia, normal Mullerian ducts, absence of Wolffian ducts, and streak gonads) who 
had Wilm's tumor when she was seven years old and was referred for the assessment of primary amenorrhea and no breast development. We sequenced the genomic DNA of all the 10 exons of the WT1 gene which mutations may happen in proposita. A new de novo insertion mutation in the 1st exon was found. A $T$ is replaced by $G$ in codon 558 at position 139, resulting in the replacement of the phenylalanine by the serine and creation of a stop codon and a truncated protein. This insertion mutation has not been described earlier and was not detected in the patient's parents and her male siblings and other 25 normal controls. The present data provide further evidence to support the functional importance of WT-1gene in sex differentiation.

\section{XY Sex Reversal with Hypertension}

\section{Cindy Ho Wei-Li, Yvonne Lim Yijuan, and Loke Kah Yin}

\section{University Children's Medical Institute, National University Hospital, Singapore}

Patients with $46 \mathrm{XY}$ sex reversal are occasionally encountered, and possible differential diagnoses include complete androgen insensitivity syndrome, complete gonadal dysgenesis, testosterone biosynthetic defects, and 17 alphahydroxylase deficiency. We report a "girl" who had discordance between the prenatal $46 \mathrm{XY}$ karyotype performed through amniocentesis and the female genitalia noticed at birth. She was initially erroneously thought to have androgen insensitivity syndrome at birth, as there was some testosterone production with the HCG stimulation test although there were no common mutations discovered on screening the androgen receptor gene. She subsequently presented with gastroenteritis at the age of 9 years and was found to have persistent hypokalaemia associated with hypertension (BP: 135/90 mm Hg). Biochemical investigations revealed markedly elevated levels of plasma ACTH, serum progesterone, deoxycorticosterone, corticosterone, and aldosterone levels, which were consistent with 17 alpha hydroxylase deficiency, a rare cause of congenital adrenal hyperplasia resulting in female phenotype in a $46 \mathrm{XY}$ genetically male individual. Genetic analysis confirmed a known homozygous deletion of codon 487-489 in the CYP17 gene in the propositus, and the parents of the propositus were found to be carriers of the mutation. She was treated with hydrocortisone with normalisation of her blood pressure and hypokalaemia and improvement of her biochemical parameters. She also underwent surgical removal of her testes with refashioning of the lower vaginal tract. Histopathology of the left testis demonstrated features consistent with cryptorchid testes with suggestion of focal intratubular germ cell neoplasia. Persistent hypokalaemia should prompt an assessment of blood pressure, as associated hypertension would suggest mineralocorticoid excess. In patients with 46XY sex reversal, the presence of hypokalaemia and hypertension should suggest a diagnosis of 17 alpha-hydroxylase deficiency. Early diagnosis is essential so as to treat the hypertension and hypokalaemia and prevent their sequelae and to allow for early surgical removal of the testes which are at risk of malignancy.

\section{Molecular Detection of $X$ or $Y$ Sequences in Turner Syndrome Patients with 45, $X$ or Those with Marker Chromosome}

\author{
Jun Fen Fu, Yang Li Dai, Fang Hong, Shan Xu, \\ and Zheng Shen
}

\section{The Children's Hospital Of Zhejiang University School Of Medicine}

The significances of the detection of hidden $\mathrm{X}$ or $\mathrm{Y}$ sequences in Turner syndrome (TS) were as follows: the issue of hidden mosaicism related to fetal survival in $45, \mathrm{X}$ patients, and the risk of gonadoblastoma was associated with the presence of $\mathrm{Y}$ sequences in TS patients. The aim of this study was to detect $\mathrm{X}$ or $\mathrm{Y}$ sequences in $\mathrm{TS}$ patients with $45, \mathrm{X}$ or those with marker chromosome using molecular analysis. We investigated clinical findings of TS patients with gonadoblastoma and whether particular Y sequences were related to the development of virilization or gonadoblastoma. A total of 59 patients were included: $45, \mathrm{X}(n=39), 45, \mathrm{X} / 46, \mathrm{X},+$ mar $(n=18)$, and $46, \mathrm{X}, \operatorname{der}(\mathrm{X})(\mathrm{X}: \mathrm{Y})(n=2)$. The PCR using 11 primers (PABY, SRY, ZFY, TSPY, DYS270, DYS280, DYS209, DYS1, YBMY, DYZ1, and DYS249) was performed to detect $\mathrm{Y}$ sequences. The confirmation of $\mathrm{X}$ sequences was analyzed by comparing between patients and their parents using 9 or 12 highly polymorphic X chromosome microsatellites (DXS 1060, 8051, 1228, 1214, 986, 990, 1001, 1047, 8043, 1098, 993, and 991$)$. One (2.6\%) of 39 patients initially identified with 45 , X karyotype was found to be positive for $\mathrm{Y}$ sequences, but none had hidden $\mathrm{X}$ sequences. The patient with hidden $\mathrm{Y}$ sequences was revealed to have $45, \mathrm{X} / 46, \mathrm{X}$, + mar karyotype. Origin of marker chromosome in 19 TS patients including one aforementioned was as follows: $\mathrm{Y}(n=8,42 \%), \mathrm{X}$ $(n=2,10.5 \%)$, and undetermined $(n=9)$. Gonadectomy was undertaken in 6 of 9 patients with $Y$ sequences and 5 of 6 gonadectomized patients had developed virilization (3 at birth, 2 in the second decade). Two patients who developed virilization in the second decade were diagnosed with gonadoblastoma (Figure). The remaining 3 TS patients with Y sequences had been thoroughly monitored. We did not find any association of gonadoblastoma with particular $Y$ sequences, because most TS patients with Y origin of marker chromosome had SRY, TSPY, and centromeric DYS270 genes regardless of gonadoblastoma. In conclusion, we found that molecular X or Y screening has a supportive role in helping to ascertain initial karyotype. About half of marker chromosome in TS was revealed to be derived from the Y chromosome. If TS patients show progressive vilization and gonadal mass during followup, it may be an ominous sign of gonadoblastoma. Careful monitoring of TS patients with $\mathrm{Y}$ sequences who did not submit gonadectomy is mandatory. 


\section{Molecular Defects of Indonesian 5-Alpha Reductase-Deficient Patients}

\author{
Nanis Sacharina Marzuki, ${ }^{1,}{ }^{3}$ Lita Putri Suciati, ${ }^{1}$ \\ and Mewahyu Tridjaja Dewi ${ }^{1}$
}

\author{
${ }^{1}$ Eijkman Institute for Molecular Biology, Jakarta, \\ Indonesia \\ ${ }^{2}$ Department of Child Health, Faculty of Medicine, \\ University of Indonesia \\ ${ }^{3}$ Pediatric Endocrinology Chapter, \\ Indonesian Pediatric Society
}

Background. Steroid 5-alpha reductase 2 deficiency is an autosomal recessive disorder with clinical spectrum ranges from a male phenotype with hypospadia to a female phenotype with normal Wolffian structures. Over 50 different mutations, mostly missense or nonsense mutations, distributed over the gene have been reported in patients with 5 alpha-reductase 2 deficiency. Several mutations were described in specific populations, including New Guinea highlands, Dominican Republic, Turkey, and Egypt. Objective. The study was performed to characterise the molecular defects of SRD5A2 gene in Indonesian patients with clinically suspected of 5 alpha reductase deficiency. Methods. Three 46, XY DSD patients from 2 unrelated families, with clinically suspected of 5-alpha-reductase deficiency, and the parents were analysed. The DNAs were extracted from lymphocytes using standard procedures, and subsequently, the 5 exons of SRD5A2 gene were sequenced using specific primers. Results. All patients (aged 13-18 years old) presented with ambiguous genitalia, which were noted since birth, and had marked masculinisation as they entered pubertal age. Two novel mutations were detected in this series, of patients and their parents, which are p.34Fs and g.699-1 G > T. The other mutation detected was p.R227Q, which commonly described in Far East Asian population. Different from other reports, in our series three mutations were found in each patient. Whether the p.R227Q mutation is considered a polymorphism or a mutation in Indonesian population warrants further study. Conclusion. Two unreported mutations of RSD5A2 gene were detected in Indonesian 5 alpha reductase deficient patients and their parents.

\section{Persistent Mullerian Duct Syndrome with Severe Hypospadia: Possibility of a Complex Sex Disorder?}

\section{Frida Soesanti, Bambang Tridjaja, Arry Rodjani, Aman Pulungan, and Jose RL Batubara \\ Department of Child Health, Department of Urology, Medical School-University of Indonesia, CiptoMangunkusumo Hospital, Indonesia}

Background. Persistent Mullerian duct syndrome is a rare disorder characterized by the presence of Mullerian derivative in otherwise normal male. It is an autosomal recessive inherited disorder caused by the mutations of the AMH or
AMH type II receptor gene. Here, we presented a case of PMDS with severe hypospadia: is it possible for a combined sex disorder? Case. A one-year-and-two-month-old boy presenting with right nonpalpable testis, right inguinal hernia, and perineal hypospadia. The left testis was within the left scrotum. He was born and raised as a boy. Pregnancy and birth history were normal. No consanguinity or family history of disorders of sex development. No other physical abnormalities were found. Developmental milestones were normal. Karyotyping from peripheral blood revealed 46, XY. Mullerian inhibiting substance level was $15 \mathrm{ng} / \mathrm{mL}$ (ref: $48.2-$ $336 \mathrm{ng} / \mathrm{mL}$ ), Basal LH $3.6 \mathrm{mIU} / \mathrm{mL}$, FSH $0.4 \mathrm{mIU} / \mathrm{mL}$, testosterone $<2 \mathrm{ng} / \mathrm{mL}$, DHEA $<6 \mathrm{ng} / \mathrm{dL}$, and androstenedione $18 \mathrm{ng} / \mathrm{dL}$. Testosterone level, DHEA, and androstenedione after HCG test stimulation were $357.2 \mathrm{ng} / \mathrm{mL}, 56 \mathrm{ng} / \mathrm{dL}$, and $35 \mathrm{ng} / \mathrm{dL}$, respectively. Exploration during herniotomy and laparoscopy revealed remnant uterus, fallopian tube, fimbriae, and a structure mimicking ovarian. Gonadal biopsy from the right gonad revealed testicular tissue, and no ovarian tissue was found. Karyotyping examination from the right gonad revealed $96 \%$ of $46, \mathrm{XY}$ and $4 \%$ of 45 , X. He was diagnosed with 46, XY DSD with persistent Mullerian duct syndrome. He was planned to undergo surgery to remove the Mullerian duct structure and orchidopexy, continued with reconstruction of hypospadia. Conclusions. This case showed us a very rare male sex disorder of persistent Mullerian duct syndrome presenting with severe hypospadia. Further effort needs to be done to find out any possibility of other underlying etiology of sex disorder in this patient instead of PMDS alone.

\section{A Case of Congenital Lipoid Adrenal Hyperplasia}

\author{
Joanna Tung, Grace Poon, Anne Kwok, \\ Pik To Cheung, and Louis Low
}

Department of Paediatrics and Adolescent Medicine, Queen Mary Hospital, The University of Hong Kong

Introduction. Congenital lipoid adrenal hyperplasia (lipoid $\mathrm{CAH}$ ) is a rare cause of adrenal insufficiency caused by mutations in the steroidogenic acute regulatory (StAR) or P450 side chain cleavage enzyme (CYP 11A1) genes. Affected patients have impaired synthesis of all adrenal and gonadal steroid hormones, and they are all phenotypically female regardless of their genetic sex. Case Report. A Chinese term neonate with normal birth weight was noted to have generalized hyperpigmentation. She developed adrenal insufficiency with severe hyponatremia, hyperkalaemia, and metabolic acidosis within the first week of life. She had normal female external genitalia with no sign of virilization. Serum cortisol was low at $84 \mathrm{nmol} / \mathrm{L}$ with elevated serum adrenocorticotropic hormone (ACTH) up to $1635 \mathrm{pg} / \mathrm{ml}$ at the time of stress, suggestive of glucocorticoid deficiency. Aldosterone was in the high normal range of $900 \mathrm{pmol} / \mathrm{L}$, but plasma renin activity was raised to more than $20 \mathrm{ng} / \mathrm{ml} /$ hour, suggesting impairment of mineralcorticoid 
production. Serum 17-hydroxyprogesterone was not elevated at $0.6 \mathrm{nmol}$, excluding the classical congenital adrenal hyperplasia caused by 21-hydroxylase deficiency. Ultrasound showed bilateral enlarged adrenals. The condition improved with hydrocortisone and fludrocortisone replacement. She remained well until the age of 12, when she was found to have delayed puberty. Hormonal workup showed elevated gonadotrophins. Her karyotype subsequently came back to be 46XY. She underwent laparoscopy, and no uterus was found. Bilateral gonadectomy was done, and the histology showed atrophic testes with no malignant change. Testicular tissues with sertoli cells and rare spermatogonia were seen within atrophic seminiferous tubules. Epididymis and vas deferens were both present. Molecular analysis of the StAR gene showed that she was homozygous for a known nonsense mutation Q258X. Conclusion. In managing newborn with apparent female phenotype and adrenal insufficiency, karyotype should always be checked as the development of adrenal and gonad are closely related. The possibility of sex reversal and the diagnosis of lipoid CAH should be considered in such situation. The correct delineation of internal pelvic organs using reliable imaging modalities or even laparoscopy, together with careful interpretation of all clinical and laboratory findings are crucial to accurate diagnosis and subsequent management.

\section{Effect of Estradiol on Secretion of Kisspeptin from Female Rat Hypothalamus and on Sexual Maturation}

\author{
Bao Sheng Yu, ${ }^{1}$ Li Xiaonan, ${ }^{2}$ Pan Xiaoqin, ${ }^{2}$ \\ Yong quan Zhang, ${ }^{1}$ Shan $\mathrm{Ye}^{1}$ and Chen Ronghua ${ }^{2}$ \\ ${ }^{1}$ Department of Pediatric Endocrinology, \\ the Second Aff iliated Hospital of Nanjing Medical \\ University, Nanjing 210011, China \\ ${ }^{2}$ Pediatric Institiue, Nanjing Medical University, \\ Nanjing 210029, China
}

Objective. To investigate the effect of estradiol on secretion of kisspeptin from female rat hypothalamus and on sexual maturation. Methods. The 50 rats were randomizied into 5 groups: the rats of group E1 and E2 received a daily s.c. administration of estradiol ( $1 \mathrm{mg} / \mathrm{kg} /$ day) for 5 (PNDs 15$19)$ and 13 days (PNDs 15-27), respectively, the rats of group $\mathrm{C} 1$ and $\mathrm{C} 2$ received a daily s.c. administration of vehicle for 5 (PNDs 15-19) and 13 days (PNDs 15-27), respectively, and the rats of natural $35 \mathrm{~d}$ group were without any treatment. All of these rats were examined daily for imperforation of the vaginal membrane to determine age at vaginal opening (OV) until killed at PND28 (group E1 and E2, C1 and C2) and PND 35 (natural 35d group). Sample of blood and hypothalamic explants were collected. Hypothalamic explants were incubated in DMEM, 30minute preincubation period preceded the experimental use of the explants, and then incubated with fresh plain media for 6 hours and with fresh plain media plus $60 \mathrm{mM} \mathrm{KCL}$ for another 1 hours. Media and serum were frozend until assayed for kisspeptin by RIA. Results. (1) The age of OV of rats in group E1 $(25.1 \pm 1.1 \mathrm{~d})$ and E2 $(24.7 \pm 0.9 \mathrm{~d})$ was significantly advanced in comparision to the natural $35 \mathrm{~d}$ group $(33.8 \pm 1.0 \mathrm{~d})$. (2) The secretion of kisspeptin $(\mathrm{pg} / \mathrm{ml} / \mathrm{h})$ from hypothalamus in group E1 $(4.31 \pm 0.72)$ and E2 $(4.10 \pm 0.17)$ was higher than those in group C1 $(3.44 \pm 0.34)$ and $\mathrm{C} 2(4.31 \pm 0.72)(P<.05)$, but there was no difference in secretion of kisspeptin between E1, E2 and the natural 35d group $(P>.05)$. All groups responded to $60 \mathrm{mM} \mathrm{KCL}$ with significantly increase in kisspeptin secretion. (3) The serum estradiol concentration in group $\mathrm{E} 1, \mathrm{E} 2$, and $\mathrm{E} 2$ was higher than those in group $\mathrm{C} 1, \mathrm{C} 2$, and E1, but again without statistically significant $(P>.05)$. Conclusion. External estradiol used in a specific development period in female rat may induce sexual maturation, which are involved in the increased secretion of kisspeptin from hypothalamus.

\section{A Case of SRY-Negative Ovotesticular Disorder of Sexual Development}

\author{
Yvonne Lim Yijuan, Cindy Ho Wei-li, \\ and Loke Kah Yin \\ University Children's Medical Institute, \\ National University Hospital, Singapore
}

Ovotesticular disorders of sexual development (DSD) (formerly known as true hermaphrodites) are very rare. More than 400 cases have been reported worldwide. Despite the well-known fact that the SRY gene is known to initiate testis formation and subsequent male differentiation in mammals, about $20 \%$ of the 46 XX ovotesticular DSD lack the SRY gene. A baby was noted to have ambiguous genitalia at birth, consisting of a phallus measuring $2.5 \mathrm{~cm}$ in length, with a diameter of $1 \mathrm{~cm}$, associated with a single urethral opening at the tip of the phallus, and a right palpable gonad. Exploratory laparotomy revealed a left streak gonad histologically proven as an ovary, a left fallopian tube, and a left hemiuterus, associated with a right gonad in the right hemiscrotum, which was histologically proven to be a testis. The chromosomal karyotype was $46 \mathrm{XX}$ and the SRY gene was absent in both the peripheral blood and both gonads, confirming the diagnosis to be $46 \mathrm{XX}$ SRY-negative ovotesticular DSD. The child was reared as a girl, with surgical removal of the testes and male internal structures. The female structures were left intact. $46 \mathrm{XX}$ SRY-negative ovotesticular disorders of sexual development can be caused by unrecognized genetic mosaicism in the gonad (which was excluded on chromosomal karyotyping of both gonads), or a mutation of an autosomal gene which permits testicular differentiation without the SRY gene. Lack of the SRY gene despite virilisation in this case indicates that other candidate genes are involved in male differentiation, of which one possibility is the SOX9 gene. Duplication of the SOX9 gene has been suggested in a case of 46 XX SRYnegative ovotesticular DSD and campomelic dysplasia, which was not present in our patient. Another case report describes a family of XX sex reversal in the absence of SRY or a SOX9 mutation, suggesting a mutation at a sex-determining 
locus other than SRY and SOX9 as the cause for the XX sex reversal trait. It is clear that normal testicular development involves an exquisitely regulated network of gene expression and protein-protein interactions and that further research may uncover the other candidate genes which are responsible for male sexual differentiation.

\section{Disorders of Bone Calcium and Phosphate Metabolism}

\section{Etiology of Nutritional Rickets: A Case Control Study}

Anju Seth, ${ }^{1}$ Varun Aggarwal, ${ }^{1}$ Raman K Marwaha, ${ }^{2}$ Satinder Aneja, ${ }^{1}$ and Pitamber Sonkar ${ }^{1}$

${ }^{1}$ Department of Pediatrics and Radiodiagnosis*, Kalawati Saran Children's Hospital and Lady Hardinge Medical College, New Delhi-110001, India

${ }^{2}$ Department of Endocrinology, Thyroid Research Centre, Institute of Nuclear Medicine \& Allied Science (INMAS), New Delhi

Objective. To study the role of calcium and vitamin D deficiency in etiology of nutritional rickets in young children. Design. Case control study. Introduction. Rickets is conventionally thought to be due to vitamin D deficiency. Studies from some tropical countries have postulated low dietary calcium as the cause of nutritional rickets. Both Vitamin D and dietary calcium deficiency are highly prevalent in India. Information on their relative contribution in the etiology of rickets in Indian children is limited. Methods. We compared 67 children with nutritional rickets with 68- age and sexmatched healthy controls. The baseline demographic (nutritional status, sun exposure (UV score), dietary calcium, and phytate intake) and biochemical parameters (serum calcium, inorganic phosphate, alkaline phosphatase, $25(\mathrm{OH}) \mathrm{D} 3$, and parathyroid hormone) were compared among the cases and controls. Results. Mean ( \pm S.D.) intake of calcium was found to be significantly lower in cases as compared to controls $204.71 \pm 129.04$ versus $453.27 \pm 234.1 \mathrm{mg} /$ day, $(P<.0001)$. The diet of cases also had a significantly lower dairy calcium and higher phytate content as compared to the controls. Mean serum 25(OH) D3 levels in both cases and controls were in the range of mild deficiency $15.94 \pm 12.43 \mathrm{ng} / \mathrm{ml}$ and $19.9 \pm 13.4 \mathrm{ng} / \mathrm{ml}$, respectively, $(P=.08)$. There was no significant difference in the mean serum 25(OH) D3 levels and UV score among the cases and controls. In cases with rickets, regression analysis showed a significant correlation ( $r=-0.279, P=.0278)$ between dietary calcium intake and radiological score used to quantify severity of rickets. Conclusions. The results of the study indicate that while vitamin $\mathrm{D}$ deficiency is prevalent in young children, its prevalence is not different among cases of rickets and healthy controls. On the other hand, dietary calcium, especially dairy calcium intake, is significantly lower among children with rickets as compared to controls. Thus, it seems that in our population, rickets develops when calcium deficiency is superimposed upon a low/borderline Vitamin D status.

\section{Clinical Presentation of Primary Hyperparathy- roidism in Adolescent Age Group}

\author{
Krishna Biswas, Richa Arora, Divendu Bhushan, \\ Shruti Sharma, Vindu Amitabh, A.S. Chawla, \\ and Rohini C.S. Bal
}

V.M. Medical College \& Safdarjang Hospital

Primary hyperparathyroidism is an uncommon condition in pediatric and adolescent age group. Here, we report 4 such cases who presented between 13-16 years of age in last 2 years. All the four of them are male. The first patient, aged 13 years, presented with repeated episodes of pancreatitis followed by multiple bony lytic lesions, particularly involving the neck of femur making him unable to walk. The second patient, aged 14 years, presented with generalized bodyache followed by an episode of acute abdominal pain suggestive of pancreatitis. Serum amylase was raised, and serum calcium was $11.5 \mathrm{mg} / \mathrm{dl}$. The third patient, aged 16 years, had multiple joint pain with difficulty in walking. He was investigated as radiological features show multiple lytic bony lesions, particularly involving the neck and shaft of the femurs. The fourth patient, aged 13 years, presented with short stature and found to have multiple renal calculi on screening ultrasound. The serum calcium was more than $11 \mathrm{mg} / \mathrm{dl}$ in all the 4 cases going as high up to $15 \mathrm{mg} / \mathrm{dl}$ on repeated testing. Serum PTH were $636 \mathrm{pg} / \mathrm{ml}$, $2696 \mathrm{pg} / \mathrm{ml}, 1274 \mathrm{pg} / \mathrm{ml}$, and $118 \mathrm{pg} / \mathrm{ml}$, respectively (normal range (15-68)). Parathyroid adenomas were localized by $99 \mathrm{~m}$ Technetium Sestamibi scan in all four. In patients nos. 1 and 3 , who had predominantly bony lesions, $25(\mathrm{OH})$ vitamin $\mathrm{D}$ were low. Postoperatively, all patients went to hypocalcaemia and required calcium supplementation. However, in patient no. 1, the bony lesion did not disappear completely even after 1 year of calcium and vitamin D supplementation. Though asymptomatic hyperparathyroidism is common in adult age group, in pediatric and adolescent age group, the clinical manifestations are prominent and varied. An early index of suspicion and investigations made accordingly leads to prompt diagnosis.

\section{Study on Effect and Mechanism of Vitamin A Deficiency on Skeletal Development of Male Growing Rats}

\author{
Xing Jie, Zhang Li, and Tian Xin \\ The Second Hospital of Jilin University Pediatrics \\ (Jilin Changchun) 130041
}

Objective. To investigate the effect and mechanism of vitamin A deficiency on skeletal development of male growing rats. Methods. 24 male Wistar rats of 21-23 days after birth were randomly divided into 3 groups, severe vitamin A deficiency (SVAD) group, marginal vitamin A deficiency (MVAD) group, and control group $(\mathrm{CN})$. All rats were fed 10 weeks. Level of vitamin A in serum was determined using the HPLC method, while levels of growth hormone $(\mathrm{GH})$ insulin-like 
growth factor (IGF-1) were detected with radioimmunoassay. The femur length of all rats was measured and modified. Gomori special staining was used to establish the cartilage morphology of femoral growth plate. Results. (1) Levels of serum vitamin A of 3 groups had statistically differences $(P<.01)$. The levels of serum vitamin A of SVAD group were significantly lower than those of $\mathrm{CN}$ group and MVAD group, and MVAD group was significantly lower than the CN group. (2) The femur length of SVAD group was lower than that of $\mathrm{CN}$ group and MVAD group whereas there were no statistical differences between MVAD group and $\mathrm{CN}$ group (P0.05). (3) GH and IGF-1 levels in serum were lowered due to the lack of VA in SVAD group, MVAD group, and $\mathrm{CN}$ group; $\mathrm{GH}$ and IGF-1 levels of SVAD group were significantly lower than those of CN group and MVAD group $(P<.01, P<.05)$. (4) Epiphyseal cartilage cell in $\mathrm{CN}$ group had obvious proliferation and differentiation, clear structural levels, large cartilage lacuna, and more common mitotic. However, SVAD group had a smaller hypertrophic zone, a relatively extended still area, disorder structure, small cartilage lacuna, and unclear showed cartilage capsule. Also, it had more dysplasia cartilage cells, most of which were naive and less obvious mitotic. Less distinct bone formation could be seen, while bone marrow fibrosis could be observed. But that situation of MVAD group was improved. Conclusions. VAD may affect GH secretion of the anterior pituitary eosinophils, and then the liver synthesizes decreased IGF-1, resulting in endochondral bone synthesis disordered.

\section{Genotype and Phenotype Findings in Childhood Onset Hypophosphatasia in Chinese: A Case Series}

\section{Anne Kwok, ${ }^{1}$ Oliver Ma, ${ }^{2}$ Simon Siu, ${ }^{2}$ Pik To Cheung, ${ }^{1}$ Grace Poon, ${ }^{1}$ Joanna Tung, ${ }^{1}$ Sidney Tam, $^{2}$ and Louis Low ${ }^{1}$ \\ ${ }^{1}$ Department of Paediatrics and Adolescent Medicine, Division of Clinical Biochemistry, Department of Pathology ${ }^{2}$ Queen Mary Hospital, The University of Hong Kong}

Introduction. Hypophosphatasia is an inborn metabolic bone disease caused by a loss-of-function mutation in ALPL gene, which encodes the enzyme tissue-nonspecific alkaline phosphatase (TNSALP). TNSALP deficiency results in substrate accumulation (including phosphoethanolamine, pyridoxal5 'phosphate, and inorganic pyrophosphate), affecting both skeletal and dental mineralization. Hypophosphatasia varies greatly in clinical presentations and is classified into 6 forms according to age at diagnosis and clinical severity. More than 200 mutations are reported. Such a great allelic heterogeneity may contribute to the wide variability of clinical manifestations. Methods. We describe the clinical picture and ALPL gene mutations in 4 Chinese patients with childhood onset hypophosphatasia. Case Reports. Patient 1 is a 3.5-year-old Chinese boy. He presented with exfoliation of deciduous teeth at 16 months of age. His mother had history of mandibular pain and shed her permanent teeth at 20 years of age, and this case was labeled as periodontitis.
His maternal grandfather, granduncles, and grandaunts had all of their teeth shed by their 30-40 s. Patient 1 was well otherwise, with height and weight both between the 75th to 90th percentile. He did not have any bone pain, deformity, or any history of fracture. But his skeletal X-ray at 3 years of age already showed osteopenia. Patients $2-4$ were siblings. Patient 2 was the index patient. He was 4 years and 10 months old at the time of study. He first presented with exfoliation of deciduous teeth at 12 months of age. In retrospect, his 7-year-old elder brother (patient 3) also shed his deciduous teeth early since 4 years of age. Patient 4 , his 16-month-old younger sister, was diagnosed subsequently by screening at 18 months of age. She was totally asymptomatic so far. All 3 sibs enjoyed normal growth and development. Both their height and weight were at 75 th percentile. They did not suffer from any bone pain, deformity, or fracture. Father was asymptomatic. But paternal grandfather reported shedding of all his permanent teeth in his $30-40 \mathrm{~s}$. He was previously diagnosed to have low bone mineral density and was advised to do weight-bearing exercise and increase calcium intake. All patients had low serum ALP, high plasma pyridoxal $5^{\prime}$-phosphate and urine phosphoethanolamine, and ALPL gene mutations (Table 5). Patients 2-4's father and paternal grandfather also carry the same c.346 G > A heterozygous mutation. Conclusions. Premature exfoliation of deciduous teeth and low serum ALP should alert clinicians to the possibility of hypophosphatasia. Genetic diagnosis and family screening is important to identify undiagnosed affected family members.

\section{Experience of Intravenous Pamidronate Treatment for Children with Osteogenesis Imperfect}

\section{Yasuhiro Naiki, Masahiro Noda, Satsuki Nishigaki, Yusuke Mizno, Kazuyuki Waki, and Reiko Horikawa}

\section{National Center for Child Health and Development}

Bisphosphonate is an only effective treatment for osteogenesis imperfecta (OI) because of its suppression effect on osteoclast. We treated 8 patients who suffered from OI (7 of type III and 1 of type IV) with intravenous pamidronate and evaluated its effects on bone and growth. 8 patients (mean age at initiation of treatment, 2 year 1 month old: range, 1 month to 6 year 5 month old: 5 boys and 3 girls) during 38 months (11 to 69 months) of pamidronate treatment. The intervals of the treatment were one month except for a case of neonate, which interval is two months. All patients but type IV decreased frequency of bone fracture without severe adverse event even if treatment is started as early as 1 month of age. During this treatment, BMD increased in all patients but was not normalized except for one case. The changes of bone markers were significant statistically. Age-matched SD score of height was worsen through therapy in one case without change of IGF-1. Renal calculus was observed in one case. Our experience suggests that cyclical pamidronate treatment is effective for patients even in neonatal period. 
TABLE 5

\begin{tabular}{lcccc}
\hline Patient & ALP* & P5P* & PEL:Cr* & ALPL gene $^{\wedge}$ \\
\hline 1 & 95 & 736 & 52.1 & a novel sequence change-significance to be confirmed \\
2 & 90 & 256 & 63.3 & heterozygous mutation for c.346 G $>$ A, p.A99T in exon 5 \\
3 & 94 & 166 & 38 & heterozygous mutation for c.346 G $>$ A, p.A99T in exon 5 \\
4 & 94 & 292 & 72.6 & heterozygous mutation for c.346 G $>$ A, p.A99T in exon 5 \\
\hline
\end{tabular}

* ALP: serum alkaline phosphate-normal: 145-420 U/L

* P5P: plasma pyridoxal 5' -phosphate-normal: $<109 \mathrm{nmol} / \mathrm{L}$

*PEL: Cr: urine phosphoethanolamine to creatinine ratio-normal: $2-17 \mathrm{umol} / \mathrm{mmol} \mathrm{Cr}$

$\wedge$ ALPL gene analysis was performed by PCR-based direct sequencing of ALPL gene on peripheral blood leucocyte-derived DNA.

\section{Report of a Rare Case of Vitamin D-Dependent Rickets Type II}

\section{Hooi Leng Ooi, ${ }^{1}$ Loo Ling $\mathrm{Wu},{ }^{1}$ Johari Mohd $\mathrm{Ali}^{,}{ }^{2}$ and Ali Saber Hossein Abadi ${ }^{2}$}

\author{
${ }^{1}$ Paediatric Endocrinology Unit, Department of \\ Paediatrics, University Kebangsaan Malaysia Medical \\ Centre, Jalan Yaacob Latif, Bandar Tun Razak, \\ 56000 Cheras, Kuala Lumpur, Malaysia \\ ${ }^{2}$ Department of Molecular Medicine, Faculty of Medicine, \\ University of Malaya, 50603 Kuala Lumpur, Malaysia
}

Introduction. Vitamin D-dependent rickets type II (VDDR II) is a rare autosomal recessive disorder caused by mutations in the vitamin $\mathrm{D}$ receptor (VDR) gene that results in resistance to the active hormone 1, 25-dihydroxyvitamin D3. We report a nine-year old girl with refractory rickets and alopecia. She had markedly elevated 1,25-dihydroxyvitamin D3 but low 25 hydroxyvitamin D level. The diagnosis of VDRR II was confirmed by molecular analysis of the VDR gene. Case Report. AS, nine-year old girl, is the fourth progeny of a first cousin marriage. She was born term with birth weight of $3.3 \mathrm{~kg}$. At one month of age, she went through cultural ritual of hair shaving and subsequently noted absence of hair growth. Patient had no eyebrows but eyelashes were present. Despite an adequate diet, AS failed to thrive. She walked unaided at two years old but she refused to bear weight from age of three. When first presented at 4.5 years old, she had features of active rickets with rickety rosary, bilateral wrist and ankle swelling, bowing of both femurs with genu valgum. Radiography showed diffuse osteopenia and classical changes of rickets. Biochemical studies revealed hypocalcaemia, hypophosphataemia, markedly elevated alkaline phosphatase and parathyroid hormone. $25(\mathrm{OH})$ Vit D level was $15.6 \mathrm{nmol} / \mathrm{l}(60-160)$ and 1, 25-dihydroxyvitamin D3 was $442 \mathrm{nmol} / \mathrm{l}$ (50-160). She was started on Rocaltriol and calcium supplements. Unfortunately, she defaulted followup for three years but continued to receive the same medications from a local hospital. She became stronger and started walking again at six years old. However, the rickety features persisted with only slight improvement in biochemical parameters. Currently, she was on high dose of one alpha calcidol, calcium supplement and Vitamin D. Molecular analysis of the VDR gene revealed a homozygous missense mutation R391C in exon 9 of the ligand-binding domain (LBD). This mutation had been reported not only reduced the ability of LBD to bind with 1,25-dihydroxyvitamin D3 but also impaired the subsequent step of VDR heterodimerization with retinoid $\mathrm{X}$ receptor (RXR). Without VDR-RXR heterodimer, the ensuing activation of Vitamin D Response Element (VDRE) of the target genes is impaired. Conclusion. VDDR II is a rare disorder. Presence of alopecia is associated with more severe degree of vitamin $\mathrm{D}$ resistance. The concomitant presence of vitamin D deficiency with VDDR II, though rare, had been reported. In view of this specific mutation, it remains a therapeutic challenge as patient is yet to respond fully to treatment.

\section{Hyperplastic Callus Formation in Osteogenesis Imperfecta Type V}

\section{Hooi Leng Ooi and Loo Ling Wu}

Paediatric Endocrinology Unit, Department of Paediatrics, University Kebangsaan Malaysia Medical Centre, Jalan Yaacob Latif, Bandar Tun Razak, 56000 Cheras, Kuala Lumpur, Malaysia.

Introduction. Osteogenesis imperfecta (OI) is the most common hereditary bone disease resulting in osteoporosis in childhood. It is characterized by bone fragility and reduced bone mass that results in multiple fractures with deformities. OI type $\mathrm{V}$ is a newly described form of brittle bone disease since 2000. One of the hallmarks of OI type V is hyperplastic callus (HPC) formation at fractures and osteotomy sites. We report a 10-year-old girl with OI type V complicated with recurrent HPC formations over the years. Case Report. SA, 10-year old girl, was first seen in our clinic at four years old with history of recurrent fractures since birth. She was born at term via spontaneous vaginal delivery with birth weight of $3.25 \mathrm{~kg}$. She had fractures of right humerus and bilateral clavicles at birth. She failed to thrive and subsequently noted to have scoliosis. She had no blue sclera, dentinogenesis imperfecta or family history of bone disorders. Her biochemistries were normal but vitamin D level was not done. Radiography showed generalised osteopenia with L3 compression fracture. She was started on cyclical intravenous pamidronate since 5 years 8 months old. Over the years, there were three episodes of long bone fractures that resulted in HPC formation, that is, right humeral fracture at birth, left femoral fracture at 2.5 years and right femoral fracture at 7 years 10 months old. HPC formation 
at birth and 2.5 years only evidence on radiographs, but the right femoral HPC formation at 7 years 10 months resulted in severe right thigh swelling which was warm and tender with normal overlying skin. It was associated with pain and reduced mobility. Initially, the HPC was enlarging and alkaline phosphatase was elevated. Subsequently, it stabilised but serial radiographs over two-year period showed minimal sign of callus regression despite improvement in pain and size of swelling. During this period, patient continued to receive pamidronate therapy. Conclusion. HPC formation, though usually treated conservatively, is a potentially serious complication as it can lead to significant morbidity. Natural history of HPC was not well understood; some regressed completely but some could progress to result in alteration of bone architecture and subsequent bone deformity. In view of minimal regression, longer followup is required to determine the final outcome of this patient. The role of pamidronate in HPC and its healing process need to be further elucidated.

\section{Hypercalcemia Complicating Malignant Rhabdoid Tumor}

\section{Catherine Anne G. Pangilinan \\ University of the Philippines-Philippine General Hospital}

Hypercalcemia complicating malignancy is a frequent finding but little is known regarding its occurrence among children. Acute leukemias, Hodgkin's Disease, non-Hodgkin's Lymphoma, and certain solid tumors such as rhabdomyosarcoma, rhabdoid tumor, hepatoblastoma, and neuroblastoma have been associated to it. Clinical manifestations of hypercalcemia usually occur late in the course with the tumor already known in most cases. Cancer-associated hypercalcemia may be due to elaboration of humoral factors produced by tumors affecting the calcium metabolism in the bone, kidney, and intestines with the Parathyroid Hormonerelated Protein (PTH-RP) being thought to play a major role. A 2.5-year-old girl with a large posterior thoracic wall mass admitted for surgery was referred for evaluation of hypercalcemia. This was associated with occasional episodes of vomiting, polydipsia, polyuria, and nocturia. This was first noted at seven months as a bean-sized, firm, nonmovable mass at the right suprascapular area which progressively enlarged. Excision of the mass was done upon consult at one year of age. Assessment made was hemangioma. No histopathologic study was done, however. Six months later, there was recurrence of the mass prompting consult at our institution. Chest CT Scan revealed a large posterior thoracic wall mass confined to the subcutaneous layer measuring $13.8 \mathrm{~cm} \times 12.6 \mathrm{~cm} \times 6.1 \mathrm{~cm}$. Lab work-ups done revealed hypercalcemia (Total Calcium: $4.35 \mathrm{mmol} / \mathrm{L}, \mathrm{NV}$ : 2.12-2.52 mmol/L; Ionized Calcium $2.33 \mathrm{mmol} / \mathrm{L}, \mathrm{NV}: 1.1-$ $1.4 \mathrm{mmol} / \mathrm{L})$, hypophosphatemia $(0.35 \mathrm{mmol} / \mathrm{L}, \mathrm{NV}: 0.81-$ 1.58), normal magnesium, alkaline phosphatase, BUN, and creatinine. PTH: $65 \mathrm{ng} / \mathrm{ml} \mathrm{NV}$ : $10-40 \mathrm{ng} / \mathrm{ml}$. Urinalysis, KUB ultrasound, and skeletal survey were essentially unremarkable. ECG revealed shortened QTc. During the admission, patient was hyperhydrated with D5 0.9\% NSS at $3 \mathrm{~L} / \mathrm{BSA}$ and was later started on prednisone with no significant decrease in calcium level. Excision of the recurrent mass was subsequently done with histopathologic studies revealing malignant rhabdoid tumor. Calcium levels normalized beginning Day 2 postoperatively. Chemotherapy with vincristine, cyclophosphamide and ifosfamide was initiated. Serial determinations of total calcium were within normal range. Four months after, the back mass recurred. There was also note of a progressively enlarging metastatic mass at the left temporal area which later involved the orbital floor. The patient was readmitted at the ward five months after for vomiting. Total Calcium was elevated $(5.7 \mathrm{mmol} / \mathrm{L})$. IV hydration and furomeside were started with gradual decrease in the calcium level $(5.25,3.99,3.34$, and $2.38 \mathrm{mmol} / \mathrm{L}$ ). Chemotherapy was resumed. The patient, however, developed nosocomial pneumonia which led to her demise.

\section{Autosomal Dominant Familial Hyperparatyroidism}

\author{
Sudeep Siwach, Andrew Cotterill, Gary Leung, \\ John Cardinal, David Mclntyre, Ivan McGown, \\ Harold Jeupner, and Mark Harris
}

\section{${ }^{1}$ Mater Childrens Hospital, Brisbane}

Objective. A family is described which has a unique autosomal dominant hypoparathyroidism with normal DNA sequence for calcium sensing receptor and preproPTH gene. Case Report. The index case is a $14 \mathrm{yr} 9 \mathrm{~m}$ boy who was diagnosed to have type 1 diabetes in 1993. He developed epilepsy in 1998 and received Carbamazepine for about 1 year. No recurrence of epilepsy was noticed. On one of his visit to endocrine clinic, he complained of tremulousness and muscle cramp involving his calf muscles. Clinical examination was unremarkable. His mother, a 40-yearold lady, revealed that she was taking treatment with 1 , $25(\mathrm{OH})$ Vit D and calcium carbonate for hypocalcemia since the age of 18 years. She was first diagnosed to have hypocalcemia when she was pregnant with her first daughter. She suffered from seizures as a child. Seizures spontaneously stopped as she grew older. Index case had no clinical evidence of hypocalcemia. Biochemistry: Ionised Ca $1.04 \mathrm{mmol} / \mathrm{L}$ (1.2-1.3); PTH $1.2 \mathrm{pmol} / \mathrm{L}$ (1-7); $25 \mathrm{OH}$ Vit D $78 \mathrm{nmol} / \mathrm{L}$ (25-150). Urinary calcium excretion was $0.14 \mathrm{mmol} / \mathrm{L}$ (low) and $\mathrm{Ca} / \mathrm{Cr}<0.1$ His 1 st degree cousin, a 10-month-old baby, had similar biochemistry: ionized Ca $1.1 \mathrm{mmol} / \mathrm{L}$; PTH $1.7 \mathrm{pmol} / \mathrm{L}$ and $25 \mathrm{O}$ Vit D $99 \mathrm{nmol} / \mathrm{L}$. Seven out of 10 children are also affected and 2 maternal cousins have hypocalcemia and hypoparathyroidism. Impression. Autosomal dominant hypoparathyroidism with normal calcium-sensing receptor and prepro-PTH genes cased by Denovo mutation in this family DNA sequencing is currently being performed with pending results. 


\section{Autosomal Dominant Hypocalcemia Caused by an Activating Mutation of the Calcium-Sensing Receptor Gene}

\author{
Se Hyun Maeng, ${ }^{1}$ YoungBae Sohn, ${ }^{1}$ Sung Won Park, ${ }^{1}$ \\ Hyung-Doo Park, ${ }^{2}$ Chang-Seock Ki2, ${ }^{2}$ Mi Yeon Kim, ${ }^{3}$ \\ Hye Won Jang, ${ }^{3}$ Jae Hoon Chung, ${ }^{3}$ \\ and Dong-Kyu Jin ${ }^{1}$ \\ ${ }^{1}$ Department of Pediatrics, \\ ${ }^{2}$ Department of Laboratory Medicine and Genetics, \\ ${ }^{3}$ Devision of Endocrinology and Metabolism, \\ Department of Internal Medicine, \\ Samsung Medical Center, Sungkyunkwan University \\ School of Medicine, Seoul, Korea
}

Hypoparathyroidism is an abnormality of calcium metabolism characterized by low serum levels of parathyroid hormone in spite of hypocalcemia. The causes of hypoparathyroidism are numerous. Activating mutations in the calcium-sensing receptor (CaSR) gene are well-known causes of familial isolated hypoparathyroidism, also known as autosomal dominant hypocalcemia (ADH). Here, we describe three members of a Korean family with a heterozygous Pro221Leu mutation causing ADH. A 2-month-old male visited our clinic due to hypocalcemic seizure occurred at 10 days after birth. The serum level of calcium was $6.3 \mathrm{mg} / \mathrm{dl}$ (reference range 8.4-10.2), and phosphorus was $8.7 \mathrm{mg} / \mathrm{dl}$ (reference range 2.54.5). He had a family history of hypocalcemia. His grandfather had a history of hypocalcemic seizure with basal ganglia calcification. His father had generalized seizure caused by hypocalcemia at the age of 20 years, and his aunt had intermittent numbness and periodic paralysis started at the age of 10 years which was also caused by hypocalcemia. On physical exam, the baby appeared well without any dysmorphic feature. The laboratory test showed hypocalcemia, hypophosphatemia, decreased 1,25dihydroxycholeccalciferol, and decreased PTH. The direct sequencing of CaSR gene of all four affected family members showed a $\mathrm{C}$ to $\mathrm{T}$ transition at nucleotide 662 resulting in heterozygous Pro221Leu missense mutation in exon 3 which was reported resulting in activating mutation of CaSR. All of the four affected family members are now on treatment with vitamin $\mathrm{D}$ and calcium carbonate. It is important to distinguish hypocalcemia due to an activating mutation of CaSR from isolated hypoparathyroidism because ADH patients can develop nephrocalcinosis and renal impairment during treatment with calcium and vitamin D.

\section{Hypocalcaemia, Hyperparathyroidism, and Vitamin D Deficiency in a Case of Neonatal Mucolipodosis II}

\section{Lim Song Hai, Fabian Yap, and Rashida Vasanwala \\ KK Women and Children Hospital, Singapore}

Mucolipodosis II (ML II or I-cell disease) of neonatal-onset is well known to have characteristic bony changes mimicking rickets, which is in distinct contrast to the typical dysostosis multiplex found in those who present at an older age. Hyperparathyroidism is a common feature in all neonatalonset ML II and widely thought to be secondary to impaired calcium transport to the fetus across the placenta such that severe hypocalcemia is not a typical finding. While vitamin D deficiency is not universal, vitamin $\mathrm{D}$ is the preferred treatment to hasten the resolution of hyperparathyroidism and bone changes. However, a unique observation in neonatalonset ML II is the complete resolution of radiological "rickety" changes whether or not vitamin D treatment is instituted. We report a case of a newborn who presented with radiological evidence of rickets, hypocalcaemia, and low 25hydroxyvitamin $\mathrm{D}$, features typically seen in children with vitamin D deficiency. However, based on the severe skeletal changes at birth, we pursued potential differentials which eventually revealed an underlying diagnosis of ML II. We reexamine the approach to a newborn with "rickety" bone changes and review previous reports of neonatal-onset ML II. We conclude that hypocalcaemia secondary to vitamin D deficiency may coexist in neonatal-onset ML II, potentially delaying the diagnosis of the underlying bone disease. The role of vitamin D in this lysosomal storage osteopathy remains unknown.

\section{Two Siblings with a Novel Nonsense Mutation, p.R50X, in the Vitamin D Receptor Gene}

Vichit Supornsilchai, ${ }^{1}$ Yodporn Hiranras, ${ }^{1}$

Suttipong Wacharasindhu, ${ }^{1}$ Atchara Mahayosnond, ${ }^{2}$ Kanya Suphapeetiporn, ${ }^{3,4}$ and Vorasuk Shotelersuk ${ }^{3,4}$

\author{
${ }^{1}$ Division of Endocrinology, Department of Pediatrics, \\ ${ }^{2}$ Department of Radiology, \\ ${ }^{3}$ Center of Excellence for Medical Genetics, \\ Faculty of Medicine, Chulalongkorn University \\ ${ }^{4}$ Molecular Genetics Diagnostic Center, \\ King Chulalongkorn Memorial Hospital, Thai Red Cross, \\ Bangkok 10330, Thailand
}

We report 2 affected children with hereditary vitamin D resistant rickets (HVDRR) from the same family, one at the age of 10 years and 9 months and the other at 9 months of age. Clinical and X-ray findings were reviewed and compared to the previous reports. RNA and genomic DNA were extracted from peripheral blood leukocytes. Mutation analysis by PCR-sequencing the entire coding region of the vitamin $\mathrm{D}$ receptor (VDR) gene was performed. A homozygous $\mathrm{C}$ to $\mathrm{T}$ transition in exon 5 of the VDR gene (c.148C $>$ T) resulting in a stop codon at amino acid position 50 (p.R50X) was identified in the proband and his younger sister. Both of their asymptomatic parents were heterozygous for the mutation. The proband had most of the clinical features of HVDRR including total alopecia. The infrequent features including a later onset of hypocalcemic symptoms and normophosphatemia were also observed. In conclusion, we report two siblings with a novel nonsense mutation, p.R50X, in the VDR gene. Hypocalcemic symptoms at a later 
onset and normophosphatemia, rarely found in HVDRR were present in the proband. This study also emphasizes an important role of genetic testing for early diagnosis and genetic counseling.

\section{Effect of Glucocorticoids on \\ Bone Status in Children Suffering from Inflammatory Bowel Disease}

\author{
Sona Tomkova, ${ }^{1}$ Emese Majorova, ${ }^{2}$ Juraj Payer, ${ }^{3}$ \\ and Peter Vanuga ${ }^{4}$ \\ ${ }^{1}$ Hospital Kosice-Saca a.s, Slovakia, \\ ${ }^{2}$ Children's Faculty Hospital, Kosice, Slovakia, \\ ${ }^{3}$ Faculty Hospital of Comenius University, Bratislava, \\ Slovakia, \\ ${ }^{4}$ National Institute of Endocrinology and Diabetology, \\ Lubochna, Slovakia
}

Background. The decreased bone density is present in children with inflammatory bowel disease. The growth of bones and changes of its metabolism are influenced by malabsorption, corticosteroid treatment, by energy and protein imbalance, low level of IGF and with loss of micronutrients through bowel. Objectives. Our goal was to determine the density of bone (BMD) in children with inflammatory bowel disease and group comparison of patients with corticosteroid treatment and without of corticosteroids. We performed prospective cohort study in 50 children (28 boys and 22) girls, mean age of this group was 13,8 years. We followed up this group for 7 years, and at least 2 densitometry measurement was performed. Methods. Lumbar spine mineral density (BMD) was assesed by Dual Energy X-ray Absorptiometry (DEXA, Hologic). Low density was considered as: $-2,0$ and more $\mathrm{Z}$ score. The results of measurement was compared with reference values adjusted for age and gender and explicated as $\pm 2 \mathrm{SD}$. The bone age was determinated according with Tanner W2, as Xray of left wrest. As bone markers vitamin D3, Ca, ionized $\mathrm{Ca}$, crossinks telopeptide of collagen I were examinated, the Pearson correlation and single and pair T-Test were used for statistical analysis. Results and Conlusion. Bone mineral density was significantly reduced in both groups (boys and girls) comparing to reference values. No statistical difference was found between treated and untreated groups (corticosteroids). Comparison of BMD versus CTx showed strong statistical significance among groups. Majority of bone markers were in normal value for gender and age.

\section{Cases of Osteogenesis imperfecta Type V-to Treat or not to Treat?}

Joanna Tung, ${ }^{1}$ Peter Hui, ${ }^{2}$ Grace Poon, ${ }^{1}$ Anne Kwok, ${ }^{1}$ Pik To Cheung, ${ }^{1}$ Wendy Lam, ${ }^{2}$ and Louis Low ${ }^{1}$
${ }^{1}$ Department of Paediatrics and Adolescent Medicine, Queen Mary Hospital, The University of Hong Kong

${ }^{2}$ Department of Radiology, Queen Mary Hospital,

The University of Hong Kong

Introduction. Osteogenesis imperfecta type $(\mathrm{OI}) \mathrm{V}$ is a distinct clinical entity with its unique clinical, radiological and histological features. Case Report. We report 2 Chinese children with OI type V. Both of them presented with repeated episodes of bony fractures since early childhood and both had the classical radiological findings of $\mathrm{OI}$ type $\mathrm{V}$ with calcification of the radioulnar interosseous membrane and subluxation of the proximal radioulnar joint. They had absence of blue sclera, normal hearing, and normal dentition. One of them continued to suffer from recurrent bone fractures after puberty. He was started on intravenous pamidronate treatment every 3 to 4 monthly for 1 year, and he had no further fracture since the commencement of treatment. The other patient had repeated fractures since early childhood but his condition improved since the onset of puberty. He developed painful hypertrophic callus 3 months after anterior closing wedge osteotomy on his right distal femur at the age of 13 years. Pamidronate treatment was not started in view of uncertain effect of antiresorptive agents on the natural history of hypertrophic callus, and the relatively mild disease at the time. Conclusion. Although bisphosphonates treatment is a well-established therapy for patients with osteogenesis impefecta type I, III, and IV, there is limited information on its effect on OI type V, particularly in patients with hypertrophic callus. Its clinical benefits should be weighed against its potential complications in every individual case.

\section{Pamidronate Use in Children with Bone Disorders}

\section{Joanna Tung, Grace Poon, Anne Kwok, Pik To Cheung, and Louis Low}

\section{Department of Paediatrics and Adolescent Medicine, Queen Mary Hospital, The University of Hong Kong}

Introduction. The use of intravenous pamidronate therapy has been suggested in a variety of bone disorders in children. The main objective of the study is to assess the effect of pamidronate treatment in Chinese children with bone disorders. Methods. A retrospective chart review was undertaken on paediatric patients who received pamidronate treatment in a single centre. 11 patients ( 3 male, 8 female) were included in the study and their average age was 10.6 years (3.4 to 16.3 years) at the start of treatment. 4 patients that had idiopathic juvenile osteoporosis, 2 with osteoporosis pseudoglioma syndrome, 2 with fibrous dysplasia, 1 with steroid induced osteoporosis, 1 with epilepsyrelated osteoporosis, and 1 with osteogenesis imperfecta were included. These children had either a history of recurrent bone fractures or nerve roots encroachment by dysplastic bones. Intravenous pamidronate was administered 
at a dose of $1 \mathrm{mg} / \mathrm{kg} /$ dose for 3 consecutive days at 3 to 7-month intervals, over a period of 1.5-7 years. Their clinical status, biochemical characteristics including bone turnover markers, and their bone mineral density were assessed regularly during treatment. Results. Serial dual Xray adsorptiometry (DEXA) scan was performed in 10 out of the 11 subjects. The bone mineral densities were all below normal at presentation with a z-score ranging from 1.5 to 5.8 SDS. Significant improvement in bone mineral densities was observed, with a mean annualized percentage gain of $9.49 \% \pm 6.12 \%$, which is beyond the expected increase of $3-5 \%$ per year in normal growth. In addition, a decrease in fracture was observed in patients who had recurrent fractures. One patient with fibrous dysplasia presenting with nerve root encroachment had increased in bone mineral density but she suffered from another episode of nerve root encroachment despite treatment. Delayed bone healings after lower limb osteotomy was observed in 2 patients with juvenile idiopathic osteoporosis. No delayed bone healing after fractures was observed. The patients otherwise experienced few adverse effects, and the side effects were limited to mild acute phase reaction during the first infusion. Conclusion. Intravenous pamidronate treatment is safe and effective in children with primary osteoporosis and secondary osteoporosis in decreasing fracture incidence and increasing bone mineral density in the first 3 to 4 years of treatment. Our experience on its use in fibrous dysplasia is not conclusive.

\section{Clinical Followup of 8 Cases of Osteogenesis Imperfecta Treated with Bisphosphonates}

\section{Veena Nair, Preeti Dabadghao, and Vijayalakshmi Bhatia}

\section{Department of Endocrinology, Sanjay Gandhi Postgraduate Institute of Medical Sciences, Lucknow, India}

Background. Osteogenesis imperfecta (OI) is a genetic disorder of increased bone fragility with severe long term morbidity and poor quality of life. Physical rehabilitation and orthopedic care are the mainstay of management along with bisphosphonate therapy. Objective. To review the clinical outcome in 8 children with moderate to severe OI treated with bisphosphonates. Methods. Case records of children with OI during the last 10 years were reviewed. Radiological imaging was done in all cases. Baseline BMD could be done only in 4 children and followup BMD in 3 due to financial constraints. Six patients were treated with cyclic pamidronate infusion and 2, for logistic issues, with oral Alendronate. Orthopedic and physiotherapy inputs were suboptimally available. Results. Total 8 patients ( 4 females) were followed up for a median period of 3 years (range 1-5 years). The median age of presentation was 3 years, and the onset of fractures was 22 days of life. Fracture rates decreased, and all children showed improvement in bone pain and mobility; 3 patients underwent intramedullary roding of femur. One girl had painful femoral swelling (which was diagnosed as hyperplastic callus) and calcification of inter osseous membrane of forearm suggesting Type V OI (Table 6). Conclusions. Our patients clearly showed beneficial effect of bisphosphonate on bone pain and fragility. Those who received simultaneous orthopedic care had better functional outcome. Oral alendronate may be a feasible option when pamidronate treatment is not possible in terms of availability/affordability.

\section{Vietnamese Patients with Osteogenesis Imperfecta: Clinical Features of 104 Cases and Results of Treatment}

\author{
Dung Chi Vu, Ngoc Can Thi Bich, \\ Khanh Nguyen Ngoc, Thao Bui Phuong, \\ Hoan Nguyen Thi, Khu Thi Khanh Dung, \\ and Nguyen Thanh Liem
}

\section{National Hospital of Pediatrics, Hanoi, Vietnam}

Osteogenesis Imperfecta (OI) is a heritable disorder characterized by bone fragility due to impairment in bone quantity and quality, which may present with a wide range of disease severities. Despite its prevalence, information regarding the clinical features of this genetic disorder is lacking in Vietnam. Thus this study aimed at characterizing the clinical feature and treating of OI patients in Vietnam so that a practical correlation could be established for distinguishing different clinical subtypes of OI. Review of medical record indentified 104 patients with OI during the study period from January 2000 to October 2009. Diagnosis and classification, using the classification system of Sillence et al. were based on clinical and radiology characteristics. Treatment with biphosphonate and follow up were performed using protocol of Rauch (2003). We also analyzed the data of these patients among different subtypes, including clinical presentations, physical examination, and bone mineral density findings. There were 60 males and 44 females with age ranging from 1 day to 15 years. Among these patients, 18 were classified as type I, 8 as type II, 66 as type III, and 12 as type IV. Height SDSs were $-1 \pm 1.99$ in type $\mathrm{I},-4.47 \pm 2.39$ in type III, and $-1.6 \pm 3.17$ in type IV. The clinical feature of blue sclera was present in $89 \%$ patients of type I, $88 \%$ of type II, $86 \%$ of type III, $86 \%$ of type IV. Annual fracture rate was $4 \pm 2.9$ in type I, $9.8 \pm 6.4$ in type II, $6.1 \pm 4.3$ in type III, $4.6 \pm 3.4$ in type IV. In type IV patients, $41.6 \%$ had family history of OI, compare to $0 \%$ in type II, $16.7 \%$ in type I, and $13.6 \%$ in type III. Biphosphonate was used to treat 30 patients with an average time of $13 \pm 0.8$ months. After 3 cycles of treatment, 100\% patients had improved the radiology feature, reduced bone pain and improved in mobility. No patient experienced new fractures after 12 months of treatment. The study indicated that there is a wide range of clinical severities among Vietnamese patients with OI, and biophosphonate has effects on treatment of OI. 
TABLE 6

\begin{tabular}{|c|c|c|c|c|c|c|}
\hline $\begin{array}{l}\text { Age at last follow } \\
\text { up (years)/Sex }\end{array}$ & $\begin{array}{c}\text { Age at fracture } \\
\text { onset }\end{array}$ & $\begin{array}{c}\text { Age at bisphosphonate } \\
\text { initiation }\end{array}$ & Drug & $\begin{array}{c}\text { No. rate/year before } \\
\text { treatment }\end{array}$ & $\begin{array}{l}\text { No. rate/year } \\
\text { on follow up }\end{array}$ & Mobility \\
\hline $10 / \mathrm{F}$ & 5 days & 7 years & Alendronate & 3 & $1^{\$}$ & Sits supported \\
\hline $3.5 / \mathrm{M}$ & Birth & 45 days & Pamidronate & - & 2 & Stands supported \\
\hline $7 * / \mathrm{F}$ & 45 days & 4 years & Pamidronate & $5-6$ & 0 & Sits unsupported \\
\hline $7 / \mathrm{F}$ & 1.5 years & 5 years & Pamidronate & $1-2$ & 2 & Walks supported \\
\hline $9 / \mathrm{F}$ & 20 days & 2 years & $\begin{array}{c}\text { Alendronate/ } \\
\text { Pamidronate no. }\end{array}$ & 6 & 3 & Sits unsupported ${ }^{\S}, €$ \\
\hline $4 / \mathrm{M}$ & 3 months & 1.5 years & Pamidronate & $2-3$ & 2 & Stands supported \\
\hline $7 / \mathrm{M}$ & 22 days & 5 years & Pamidronate & $3-4$ & $0-1$ & Walks unsupported $\S$ \\
\hline $4.5 / \mathrm{M}$ & 1.5 years & 3.5 years & Pamidronate & 3 & 2 & Walks unsupported ${ }^{\S}$ \\
\hline
\end{tabular}

${ }^{*}$ Type V OI ${ }^{\$}$ : lost to followup after 3 years, ${ }^{\#}$ : on Pamidronate during last 6 months follow up.

$\S$ : underwent intramedullary roding.

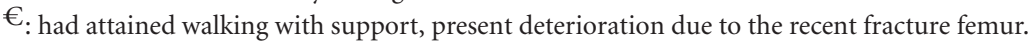

\section{A Recurrent Mutation c.617G $>A$ in the ACVR1 Gene Causes Fibrodysplasia Ossificans Progressiva in a Chinese Patient}

Chen Xiuli, Cao Lingfeng, Xi Li, Pei Zhou, Lu Wei, Zhang Miaoying, Cheng Ruoqian, Zheng Zhangqian, Shen Shuixian, Yang Yi, and Luo Feihong

\section{Children's Hospital Of Fudan University}

Fibrodysplasia ossificans progressive is a rare autosomal dominant disorder characterized by progressive heterotopic ossification and congenital malformation of the great toes. Recently, FOP has been associated with a specific mutation of ACVR1, the gene coding for a bone morphogenetic protein type I receptor. One heterozygous mutation (c.617G > A, p.R206H) in ACVR gene was identified in all sporadic and familial FOP cases with classic phenotype. We report the first Chinese uighur FOP patient with heterozygous de novo mutation of ACVR1 gene.

\section{Report of Five Chinese Patients with Progressive Pseudorheumatoid Dysplasia: From Clinical Diagnosis to Molecular Studies}

Jun Ye, Hui-wen Zhang, Wen-juan Qiu, Lian-shu Han, Ya-fen Zhang, Zhu-wen Gong, and Xue-fan Gu

The department of Pediatric Endocrinology and Genetic Metabolism, Shanghai Institute for Pediatric Research, Xinhua Hospital, Shanghai Jiao Tong University School of Medicine, Shanghai, China

Progressive pseudorheumatoid dysplasia (PPD) is a rare autosomal recessive inherited disease characterized by generalized bone-cartilage dysplasia, which has been attributed to mutations in the gene encoding WISP3. The aim of this study is to recognize PPD from clinical manifestations and imaging characteristics of bones and to investigate the gene mutations of WISP3 in Chinese patients with PPD. Five patients from five unrelated Chinese families, who presented with waddling gait, progressive swelling, pain, and restriction of movements of several joints including interphalangeal joints, visited our clinics at the age of 9-15 years old. The radiographic imaging revealed no erosive arthropathy, but platyspondyly, ovoid, or wedged anterior end-plate of vertebral bodies in all patients, coxa vara, widened epiphyses, or metaphyses including capital femoral, metacarpophalangeal and interphalangeal joints in some patients. Normal laboratory values were found for the erythrocyte sedimentation rate and C-reactive protein, rheumatoid factors, and so on, in all patients. So five patients were diagnosed as PPD according to clinical symptoms, signs and the findings of radiographic imaging. The molecular study showed five patients carry c.624_625insA/c.729_735delGAGAAAA, c.624_625insA/c.866_867insA, c.866_867 insA/c.866_867insA, Q46X/C114W, and C223G/C114W mutations, respectively, on exon $4(40 \%)$, exon $2(30 \%)$ and exon $5(30 \%)$, of the WISP3 gene. All six different mutations above found in this study have not been previously reported. The illustrative patients here serve to remind physicians to carefully examine the entire skeleton including spine and not to oncentrate only on extremities skeleton in order to prevent misdiagnosis. The mutation analysis of WISP3 gene can help to confirm the clinical and radiograph diagnosis for PPD.

\section{Hypoglycaemia}

\section{Reversal of Sensorimotor Peripheral Neuropathy and Refractory Hypertension with Surgical Cure of Insulinoma in a 10-Year-Old-Girl}

\author{
Parjeet Kaur, ${ }^{1}$ Rajesh Khadgawat, ${ }^{1}$ \\ Sandeep Agarwala, ${ }^{2}$ Bhawna Kaul, ${ }^{3}$ \\ Manjari Tripathi, ${ }^{3}$ and Ariachery C. Ammini ${ }^{1}$ \\ ${ }^{1}$ Departments of Endocrinology and Metabolism \\ ${ }^{2}$ Department of Pediatric Surgery \\ ${ }^{3}$ Department of Neurology all India Institute of \\ Medical Sciences New Delhi, India
}


Introduction. Insulinomas present rarely in children. Here we present a case of insulinoma with peripheral neuropathy and hypertension in a child. Case Report. Anu, a 10-year-old girl presented to the neurology department with history of episodic irrational behavior and seizures for 12 months and features suggestive of sensorimotor peripheral neuropathy for one and a half months Evaluation in the hospital revealed hypertension, peripheral symmetrical sensory motor neuropathy, and hyperinsulinemic hypoglycemia. MRI revealed a tumor $1.3 \mathrm{~cm}$ in size in the body of pancreas. She required multiple drugs for control of hypertension. Surgical removal of insulinoma led to resolution of hypoglycemic episodes. Drugs for hypertension could be tapered off by the 3rd month, and at one year follow up her muscle weakness has almost completely recovered. Conclusion. Reversal of hypertension and neuropathy with removal of insulinoma suggests that both these were caused by hypoglycemia, hyperinsulinemia, or a combination of both. To the best of our knowledge, this is the first case of insulinoma with neuropathy and hypertension in a child.

\section{Congenital Hyperinsulinism: A Vietnamese Case with a Novel Mutation of Abcc8 Gene}

\author{
Nguyen Ngoc Khanh, ${ }^{1}$ Vu Chi Dung, ${ }^{1}$ \\ Nguyen Thi Hoan, ${ }^{1}$ Can Thi Bich Ngoc, ${ }^{1}$ \\ Nguyen Thanh Liem, ${ }^{1}$ Khu Thi Khanh Dung, ${ }^{1}$ \\ Sarah Flanagan, ${ }^{2}$ and Sian Ellard ${ }^{2}$ \\ ${ }^{1}$ National Hospital of Pediatrics, Hanoi, Vietnam
${ }^{2}$ Molecular Genetics Laboratory, Penisula Medical School,
University of Exeter and Plymouth, UK
}

Background Congenital hyperinsulinism (CHI) is one of common causes of severe and persistent hypoglycemia in the newborn period. The incidence of sporadic and familial forms is 1 in 50000 children. As CHI is a major risk factor for brain injury and subsequent neurodevelopment handicap, the identification, rapid diagnosis and prompt management of patients with $\mathrm{CHI}$ are essential if brain damage is to be avoided. Advances in molecular genetics, radiological imagining techniques (18FDOPA-PET), and laparoscopic surgery have completely changed the clinical approach and treatment to patients with CHI. Case Report. The term girl with birth weight of $3.8 \mathrm{~kg}$ was caesarean section from the mother without any evidence of maternal hyperglycemia. She is the first child, born to nonconsanguinity parents, and had no perinatal asphyxia. 1 day after birth, she had poor feeding, convulsion, lethargy, then admitted in our hospital on the clinical condition of lethargy, poor feeding, no convulsion, no respiratory failure (SpO2: 97\%), normothermia, warm extremities, macrosomia, and no hepatospleenomegaly. The investigations showed severe hypoglycemia $(0.1 \mathrm{mmol} / \mathrm{L})$, hyperinsulinemia (217 MUI/ml), normoamonemia $(40 \mu \mathrm{g} / \mathrm{dL})$. The rest of investigations such as cortisol 8 am, arterial blood gas, transaminase, ure, creatinine, electrolyte, lactate, urine ketone, and abdominal ultrasound were unremarkable. Immediately, she was treated with glucose infusion $9-12 \mathrm{mg} / \mathrm{kg} /$ minutes, then diazoxide $(6-12 \mathrm{mg} / \mathrm{kg} /$ day) after 10 days of admission. But her blood glucose concentration had been still low (ranged 1$5 \mathrm{mmol} / \mathrm{l}$ ). Then, the patient was performed near-total pancreatectomy after 20 days of admission. We had to continue high rate of glucose infusion $(10 \mathrm{mg} / \mathrm{kg} /$ minutes $)$ to maintain normoglycemia during 2 days after the surgery. Her blood glucose level became normal level without glucose infusion after 7 days of the surgery. A compound heterozygote for two missense mutations, F686I and F686S, in exon 15 of the ABCC8 gene was identified. The T>A mutation (from her father) at nucleotide 2056 (c.2056T $>$ A) results in the substitution of isoleusine for phenylalanine at codon 686 and has not been reported previously. The $\mathrm{T}>\mathrm{C}$ mutation (from her mother) at nucleotide 2057 (c.2057T>C) result in the substitution of serine for phenylalanine at codon 686 and has been previously reported. Now, she is 3 months of age and normal development and normoglycemia. Conclusion. This is the first case of CHI in Vietnamwas analyzed ABBC8 and KCNJ11 gene. A novel mutation of ABCC8 (c.2056T>A) was identified. The autosomal recessive $\mathrm{CHI}$ result in severe and persistent hypoglycemia and often require performed pancreatectomy to maintain normoglycemia.

\section{Clinical Analysis of 40 Cases Hyperinsulinemic Hypoglycemia of Infancy}

\section{Chang Su, Chunxiu Gong, and Changhong Li}

\author{
${ }^{1}$ Beijing Children's Hospital Affiliated to The Capital \\ Medical University \\ ${ }^{2}$ The Children's Hospital of Philadelphia
}

Objective. we reviewed the diagnosis and treatment of 40 infancies with hyperinsulinemic hypoglycemia $(\mathrm{HH})$ in order to learn from $\mathrm{HH}$ and reduce misdiagnosis. Methods. Reviewing on clinical presentation, laboratory data and management retrospectively. Results. Sex ratio of boys to girls was $25: 15$. The most common chief complaint was convulsion, $92.5 \%$. $17.5 \%$ patients were misdiagnosis as epilepsy or hypocalcemic convulsions at first medical visit. $50 \%$ patients had disorders in perinatal period. $40 \%$ were large for gestational age. 58 times image studies in 40 patients did not show any positive results. We used frequent feeding combined with drug treatment to those patients. Hypoglycemic attacks reduced from up to 5 times per day to 1 time after several months, at median age of 20 months old (upper quartile 20 months old, lower quartile 14 months old). 24 were treated with diazoxide, and $13(54 \%)$ of them had good response. Followingup, 57\% (20/35) patients had mental retardation. 25.7\% (9/35) patients spontaneously remised at different ages. Conclusions. Hyperinsulinemic hypoglycemia had atypical symptoms. Misdiagnosis of $\mathrm{HH}$ was very common. Blood glucose must be tested in infants with convulsion. Any perinatal disorder and macrosomia was necessary for blood glucose and insulin checking. Hypoglycemic attack would alleviate with the age increasing. Diazoxide was effective for more than half cases. Some cases could have spontaneous resolution. Neuropathy 
complications were high. The only way to prevent central nervous system damage was diagnosis and treatment earlier. We suggest food management combined with drug treatment for patients, as well as closely blood glucose monitoring. $\mathrm{HbAlc}$ should be an indicator of this disease.

\section{An Unexpected Histological Finding of a Case of Congenital Hyperinsulinism}

\author{
Joanna Tung, Grace Poon, Anne Kwok, \\ Pik To Cheung, and Louis Low
}

\section{Department of Paediatrics and Adolescent Medicine, Queen Mary Hospital, The University of Hong Kong}

Introduction. Congenital hyperinsulinism refers to inherited disorders of dysregulated insulin secretion and it is a common cause of severe and persistent hypoglycemia in the neonatal and infancy period. Histologically, persistent disease can grossly be divided into the diffuse and focal forms. The preoperative differentiation of these two forms is important since the surgical management is entirely different and patients with focal disease can be completely cured by limited resection of the pancreas. Case Report We report a case of congenital hyperinsulinism. The child had persistent hypoglycemia despite dietary manipulations and was resistant to medical therapy with diazoxide, hydrochlorothiazide, and octreotide. Magnetic resonance imaging (MRI) did not identify any focal lesion in the pancreas but further investigation with 18 fluoro-L-Dopa positron emission tomography (PET) showed a focus of moderately intense abnormal isotope activity in the pancreatic head. Initially, limited resection of the pancreas was planned, but eventually, a $98 \%$ subtotal pancreatectomy was done due to technical reasons. Histological analysis subsequently showed a focus of adenomatous hyperplasia in the proximal one third of the pancreatic specimen but large islet cell nuclei are also seen in the other pancreatic areas, which was suggestive of diffuse hyperinsulinism. Conclusion. This case highlights the importance of intraoperative biopsy at various sites of the pancreas and careful interpretation of Dopa-PET findings in order to minimize the need of multiple surgeries.

\section{Persistent Hyperinsulinemic Hypoglycemia in the Neonate with the Mutation of the Gene ABCC8}

\author{
Jeesuk Yu, ${ }^{1}$ Ji Yoon Park, ${ }^{1}$ In Chang Seong, ${ }^{1}$ \\ Young Pyo Chang, ${ }^{1}$ Gu Hwan Kim, ${ }^{2}$ \\ and Han Wook Yoo ${ }^{2}$ \\ ${ }^{1}$ Department of Pediatrics, College of Medicine, \\ Dankook University, Cheonan, Korea \\ 2 Medical Genetics Clinic and Laboratory, \\ University of Ulsan College of Medicine, \\ Asan Medical Center, Seoul, Korea
}

Persistent hyperinsulinism is known as the most severe form of hypoglycemia in early infancy. It is especially dangerous due to the low plasma ketone as well as hypoglycemia, the condition which magnifies the risk of brain damage. Those infants with the most severe forms have a tendency to present earliest. There are several genes which cause the hyperinsulinemic hypoglycaemia including ABCC8, KCNJ11, GCK, and GLUD1. Here is a term neonate who was born with the birth weight of $4.965 \mathrm{~g}$ and gestational age of $37+5$ weeks. There was no history of maternal diabetes. Initial blood sugar test (BST) showed $25 \mathrm{mg} / \mathrm{dL}$, and it was wax and wane in spite of the continuous infusion of higher concentration of glucose and active feeding. The laboratory results were as follows: serum glucose $36 \mathrm{mg} / \mathrm{dL}$, insulin $76.1 \mathrm{uIU} / \mathrm{mL}$, c-peptide $10.1 \mathrm{ng} / \mathrm{ml}$, ACTH $187.1 \mathrm{pg} / \mathrm{mL}$ (normal 6-60), cortisol $14.2 \mathrm{ug} / \mathrm{dL}$, and free fatty acid $169 \mathrm{umoL} / \mathrm{L}$ (normal 176-586). Serum ketone was negative at that time. We started medication with diazoxide, but the response was not complete, so subcutaneous injection of the octreotide was also added. The BST became stabilized, and after then we could stop diazoxide medication. During the followup, intermittently asymptomatic low blood sugar levels were noted, but they improved very rapidly after active feeding or sometimes with glucagon injection without any severe symptomatic hypoglycaemia. We maintained frequent and active feeding every 2-3 hours and octreotide injection three times a day (initially 4 times a day). Now he is over 4 months of age, and he can control his head and is trying to roll over. Genetic screening for Beckwith Wiedemann syndrome showed no abnormality. Mutational analysis of the genes KCNJ11, GCK, and GLUD1 revealed no abnormality, but we could find one mutation in the gene ABCC8 (c. $3748 \mathrm{C}>\mathrm{T}$, p. Arg1250X). Here we report a neonate presenting as the persistent hyperinsulinemic hypoglycaemia with the mutation of the gene ABCC8.

\section{Disorders of Puberty}

\section{Precocious Puberty and Gelastic Seizures with a Hypothalamic Hamartoma}

\section{Aditiawati Anang}

\section{Endocrinology Division Departement of Child Health, Faculty of Medicine Sriwijaya University/Moh. Hoesi Hospital Palembang, Indonesia}

Background. Sexual precocity can result from primary dysfunction of the neuroendocrine system within the brain. Hypothalamic hamartoma $(\mathrm{HH})$ is a rare congenital nonneoplastic heterotopia consisting of neuron, glial cells, and fiber bundles. Clinically, most patients with $\mathrm{HH}$ present with precocious puberty and/or gelastic epilepsy. Objective. To report a rare case of hypothalamic hamartoma in 5-year 7-month old boy. Case Report. A boy with 5-years and 7month, BW $30 \mathrm{~kg}$, height $130 \mathrm{~cm}$, admitted on June 12th 2008 with main complaint hair growth in pubic area and episodes of unprovoked stereotyped laughter lasting for few seconds since 4 months old. there was stage of puberty as hair growth in pubic area with enlarged penis and large testis for his age (A1P2G3-4). There were no other neurological 
deficits, LH: $0.85 \mathrm{mIU} / \mathrm{mL}$, FSH $0.5 \mathrm{mIU} / \mathrm{ml}$, Testosterone $239,20 \mathrm{ng} / \mathrm{dL}$, FT4 $1,110 \mathrm{ng} / \mathrm{dL}$, TSH $2.228 \mathrm{IU}$. EEG is normal, bone age (Greulich and Pyle): advanced growth, appropriate with an 8-years 6-month-old boy. CT Scan: suspect tuber cinerium hamartoma. (MRI): The morphology is suggestive of tuber cinerium hamartoma. After 1 year of receiving topiramate $2 \times 20 \mathrm{mg}$ and leuprolin $3.37 \mathrm{mg}$ injection intramuscular every month, the patient was clinically improved, no tumor enlargement using MRI, and body height increase to $140 \mathrm{~cm}$. Conclusion. Hypothalamic hamartoma needs specific attention due the possibility of precocious puberty and gelastic seizures that require early management. These conditions could result in cognitive and behaviour disoders that will psycholocally impact on the family and the patient.

\section{Genetic Research Associated with Central Precocious Puberty and Timing of Puberty}

Sung Yoon Cho, ${ }^{1}$ SungWon Park, ${ }^{1}$ Young Bae Sohn, ${ }^{1}$ Se hwa Kim, ${ }^{1}$ Seung-Tae Lee, ${ }^{2,3}$ Jong-Won Kim, ${ }^{2,3}$ and Dong-Kyu Jin ${ }^{1}$

\section{${ }^{1}$ Department of Pediatrics, Samsung Medical Center, Sungkyunkwan University School of Medicine, Seoul, Korea \\ ${ }^{2}$ Center for Genome Research, Samsung Biomedical Research Institute, Samsung Medical Center, Seoul, Korea ${ }^{3}$ Department of Laboratory Medicine, Samsung Medical Center, Sungkyunkwan University School of Medicine, Seoul, Korea}

Background. Recently, variation in LIN28B was supposed as a control factor of the timing of major developmental events. And LIN28B was reported to be associated with timing of puberty in humans. Objective. The objective of the study was to investigate of genetic cause in precocious puberty and evaluate the variation in the LIN28B gene in 196 subjects with early pubertal development and compared with control subjects. Methods. For this study, 5 males and 191 females with early pubertal development were included. Control subjects were women under 30 years old, who know their age of menarche and have normal adult height. Early pubertal development was defined by appearance of breast development before the age of 9 years and in males when testicular enlargement was present before the age of 10 years. Through the literature review, we selected 22 single nucleotide polymorphisms (SNPs) which were previously reported to be significantly associated with precocious puberty with $P$-values below $5 \times 10-8$. The target SNPs were genotyped by the Sequenom MALDITOF genotyping system. Differences in genotype frequencies were statistically analyzed in dominant, recessive, genotype, and allelic modes. Result. Two SNPs near the LIN28B gene, rs2095812, and rs314268, were significantly associated with precocious puberty when analyzed in recessive mode $(P=.032$ and .024 , resp.). Of them, rs314268 was also significantly associated in genotype mode $(P=.042)$, while rs2095812 showed a borderline significance $(P=.059)$.
Conclusions. Our results show that SNPs near the LIN28B gene do underlie early pubertal development. They are associated as recessive mode, and one of them also associated in genotype mode.

\section{Primary Ovarian Dysfunction after Hematopoietic Stem Cell Transplantation during Childhood}

\section{Won Kyoung Cho, So Huyn Park, Seung Hoon Hahn, Min Ho Jung, and Byung-Kyu Suh}

\author{
Pediatrics, College of Medicine, \\ The Catholic University of Korea, Seoul, Korea
}

Background. Survival rates after hematopoietic stem cell transplantation for hematological malignancies during childhood over the past decades have advanced. And there is much concern about the effects on gonad during treatment for hematological malignancies. Limited data are available regarding ovarian failure after cancer treatment during childhood. We evaluated the incidence of and patient/treatment factors associated with ovarian failure in pediatric cancer survivors. Methods. 35 girls who were over 12 years and referred to evaluate ovarian function among patients underwent HSCT, at the Catholic HSCT Center between March, 2008 and March, 2010 were included. They were between 12.0 and 20.6 years old (average $14.7 \pm 2.1$ ) at last visit. The occurrence of menarche and basal serum follicular stimulating hormone (FSH) concentrations was assessed retrospectively. Primary ovarian failure was diagnosed if basal FSH level was $>20$ IU/L with hypoestrogenism. Results. Of thirty five girls who underwent HSCT, twenty three patients $(65.7 \%)$ developed primary ovarian failure. The interval between HSCT and last visit was $1.15-11.58$ years (average $5.91 \pm 2.94$ ). Age at HSCT was significantly older in girls with primary ovarian failure than normal $(9.62 \pm 3.05$ versus $7.12 \pm 2.23$, $P=.010)$. Chi-square showed that primary ovarian failure was less likely if patient receive a total body irradiation (patients who receive a total body irradiation compared with patients who do not, $47 \%(8 / 17)$ versus $83 \%(15 / 18),(P=$ .028 ), but there was no significant difference in Busulfan and cyclophosphamide-based chemotherapy. A univariate logistic regression analysis for detect pretransplantation risk factors revealed relations of the age at HSCT (OR: 1.395, 95\% confidence interval (CI): $(1.04-1.88, P=.028)$, TBI (OR: $0.18,95 \%$ confidence interval (CI): (0.04-0.85, $P=.030$ ), and diagnosis of myeloid leukemia (OR: 5.67, 95\% confidence interval (CI): $(1.24-25.88, P=.025)$ with primary ovarian failure. Conclusions. We observed primary ovarian failure in $65.7 \%$ of patients following HSCT and favourable prognosis of ovarian function in girls who underwent HSCT at young age. These results emphasize the regular ovarian function test of these patients in order to detect ovarian failure. The preparative regimen consisted of total body irradiation ( $n=17,12 \mathrm{~Gy}, 2 \mathrm{~Gy} /$ fraction, two fraction/day for 3 days), Busulfan-based chemotherapy ( $n=5,130 \mathrm{mg} / \mathrm{m}^{2}$ for once daily intravenously for 4 days, total $520 \mathrm{mg} / \mathrm{m}^{2}$ ), cyclophosphamide-based chemotherapy 
( $n=12,50-60 \mathrm{mg} / \mathrm{kg}$ once daily intravenously for 2-4 days, total $120-200 \mathrm{mg} / \mathrm{kg}$ ), and others $(n=1$, mephalan-based chemotherapy).

\section{Precocious Puberty in a Girl with Suprasellar Germinoma: A Case Report}

\author{
Yodporn Hiranras, Vichit Supornsilpchai, \\ and Suttipong Wacharasindhu
}

Division of Endocrinology and Metabolism, Department of Pediatrics, Faculty of Medicine, Chulalongkorn University

Primary intracranial germ cell tumor in childhood is rare and usually localized in the pineal and the suprasellar region. Although precocious puberty is common in boys with human chorionic gonadotropin (hCG) secreting brain tumor, it is extremely rare in girls. There have been few reports in which a human chorionic gonadotropin-(hCG)producing tumor associated with precocious puberty in female. We report a 7 2/12-year-old girl with suprasellar germinoma who developed isosexual precocity. The patient presented with evidence of polyuria, polydipsia, visual disturbance, ptosis and was noted to have breast bud. The serum hCG level was $243.2 \mathrm{mIU} / \mathrm{ml}$. Both luteinizing hormone $(\mathrm{LH})$ and follicle-stimulating hormone (FSH) were below detectable level. After gonadotropin releasinghormone stimulation test, $\mathrm{LH}$ and FSH remained below detectable level. Serum estradiol was elevated. The breast enlargement and bleeding per vagina were observed during admission. Pelvic ultrasonography revealed normal study. After chemotherapy, the breasts were decreased in size. The present case illustrates the development of isosexual precocious puberty in girl with hCG-producing suprasellar germinoma. It is speculated that FSH-like activity of human chorionic gonadotropin may play a role in the development of precocious puberty in this case.

\section{Onset of Secondary Sexual Maturity in Obese Boys: A Longitudinal Study}

\author{
Modella Rahmi Femina, Madarina Julia, \\ and Soeroyo Machfudz \\ Department of Child Health, Faculty of Medicine, \\ Gadjah Mada University/Dr. Sardjito Hospital, \\ Yogyakarta, Indonesia
}

Obesity was associated with early onset of sexual maturity in girls; however, results of studies in boys were conflicting. Early onset of sexual maturity is a concern because of its association with shorter final height. The objective of the study was to assess the onset of secondary sexual maturity in obese urban Indonesian boys, compared to nonobese boys as controls. The study recruited 133 obese and 133 nonobese boys in Yogyakarta, matched for age and school. Obesity was defined as BMI for age above the 95th of the CDC 2000 reference standard. All boys were between 9 and 10 years old and had not begin their secondary sexual development assessed with testicular volume of $2 \mathrm{~mL}$ or less and Tanner staging of pubic hair P1 at baseline. At baseline, 99.2\% boys had testicular volume of $1 \mathrm{~mL}$; only 2 nonobese boys had testicular volume of $2 \mathrm{~mL}$. nonobese boys were slightly taller than the obese boys, mean (SD) height of $137.9(1.8) \mathrm{cm}$ versus $136.2(8.7), P=.03$. Staging of pubic hair and testicular volume were examined every 4 months in two years, that is, 6 visits. Onset of secondary sexual maturity was defined as testicular volume of $4 \mathrm{~mL}$ or more, or the appearance of pubic hair stage P2. By the end of study, 106 (79.7\%) obese boys attained testicular volume of $4 \mathrm{~mL}$ or more versus $85(63.9 \%)$ in nonobese boys, RR (95\%CI) of 1.8 (1.2-2.7), $P=.004$. For pubic hair, 81 (60.9\%) obese boys attained P2 versus $37(27.8 \%)$ in nonobese boys, RR (95\%CI) of $1.8(1.5-2.3), P<.001$. Survival analysis showed that the median $(95 \% \mathrm{CI})$ duration for achieving testicular volume of $4 \mathrm{~mL}$ or more was 20.0 (19.3-20.7) months in obese boys versus 24.0 months in nonobese boys, $P=.007$. Since the mean age at baseline was 9.3 years, it corresponded to the age of 11.0 and 11.3 years. For pubic hair staging, the median $(95 \% \mathrm{CI})$ duration for achieving P2 was 16.0 (15.216.8) months in obese boys versus 20.0 (18.9-21.1) months in nonobese boys; it corresponded to age of 10.6 and 11.0 years. Our study concludes that obesity is associated with earlier onset of sexual maturity in boys, which may lead to shorter final height since both groups had similar heights at baseline.

\section{A Correlation between Age of Menarche and Anthropometric Measures in Adolescents and Young Women}

\author{
Hae Soon Kim, Hanwool Kim, Hyejin Lee, \\ Ji Young Oh, and Yeon-Ah Sung
}

\section{Department of Pediatrics and internal Medicine, Ewha Womans University School of Medicine, Seoul, Korea}

Purpose. To evaluate the correlation between age of menarche and anthropometric measures in adolescents and young women. Method. We surveyed 8.330 fertile women, aged between 15 and 29, to find out anthropometric measures and age of menarche. They were assorted into "adolescents" (15-18 years) and "adults" (19-29 years), then each group was categorized into "early menarche" ( 10 years), "mid menarche" (11 14 years), and "late menarche" ( $\sim 15$ years), compared with anthropometric measures. Results. The mean age was $17.2 \pm 2.4$ years of age in adolescents and $21.0 \pm$ 2.1 years of age in adults. The mean age of menarche was $12.55 \pm 1.2$ years in adolescents and $12.53 \pm 1.2$ years in adults. There was no significant difference between the two groups. The height of the two groups showed no difference $(161.8 \pm 4.9 \mathrm{~cm}$ in adolescents, $162.3 \pm 4.7 \mathrm{~cm}$ in adults; $P=.52)$. The body mass index and waist circumference were significantly different between the two groups $(20.1 \pm$ $2.4 \mathrm{~kg} / \mathrm{m}^{2}, 68.2 \pm 5.8 \mathrm{~cm}$ in adolescents; $19.8 \pm 2.0 \mathrm{~kg} / \mathrm{m}^{2}$, $67.7 \pm 4.7 \mathrm{~cm}$ in adults). In adults, the mean height of early, mid, and late menarche woman was $161.3 \pm 4.5 \mathrm{~cm}$, $162.3 \pm 4.1 \mathrm{~cm}$, and $163.8 \pm 4.9 \mathrm{~cm}$. The waist circumferences 
in those groups were estimated at $69.8 \pm 5.5 \mathrm{~cm}, 67.7 \pm$ $4.7 \mathrm{~cm}, 66.6 \pm 4.5 \mathrm{~cm}$. Also, the body mass index was $20.9 \pm$ $2.3 \mathrm{~kg} / \mathrm{m}^{2}, 19.7 \pm 2.0 \mathrm{~kg} / \mathrm{m}^{2}, 19.2 \pm 0.8 \mathrm{~kg} / \mathrm{m}^{2}$. There were significant differences among the three groups $(P<.001)$. The age of menarche in adolescents and results had a positive correlation with height, but a negative correlation with weight, body mass index and waist circumference $(P<.001)$. According to timing of menarche, prevalence of oligomenorrhea was significantly different as $6.1 \%, 8.5 \%$, $13.4 \%$ in adolescents, $5.1 \%, 7.6 \%, 15.3 \%$. Conclusion. The earlier onset of menarche is correlated with bigger waist circumference and body mass index. It suggests that the age of menarche is related to height and obesity.

\section{The Value of MRI and IGF-1 in the Differential Diagnosis between ICPP and ICPP Complicated with GH Deficiency Girls}

\section{Caihong Liu, Guimei Li, Xin Qiao, and Yanyan Hu \\ Provincial Hospital Affiliated to Shandong University, Jinan 250021, China}

Objective. To analyze the sensitive indicators of differential diagnosis between idiopathic central precocious puberty (ICPP) and ICPP associated with growth hormone deficiency (GHD). Methods. 36 ICPP girls and 16 ICPP girls associated with GHD (ICPP + GHD) were enrolled and all of them underwent magnetic resonance imaging (MRI) scans of the hypothalamic-pituitary area, the B-mode uItrasound of ovaries and uterus, bone age (BA), fasting blood IGF-1, FSH, LH, E2, To, PRL, FT3, FT4, TSH, and the peak of LH, and LH/FSH after the stimulation of GnRH, Growth hormone concentrations were measured in patients suspected with ICPP + GHD after GH stimulation test. 30 healthy prepuberty girls were selected as the control group. Results. Bone age was significantly advanced in ICPP group than in ICPP + GHD group, pituitary height, IGF-1, and the peak of LH and LH/FSH were significantly higher in ICPP group than in ICPP + GHD group, compared with the control group, pituitary height and IGF-1 in the ICPP group were significantly higher, and they were significantly lower in the ICPP + GHD group. Conclusion. MRI and IGF-1 are helpful in the differential diagnosis between ICPP girls and ICPP complicated with GHD girls.

\section{The Value of MRI and IGF-1 in the Early Diagnosis of Girls with Idiopathic Central Precocious Puberty}

\section{Yanyan Hu, Guimei Li, and Xin Qiao \\ Provincial Hospital Affiliated to Shandong University, Jinan 250021, China}

Objective. To observe the value of magnetic resonance imaging (MRI) and insulin-like growth factor-1 (IGF-1) in the early diagnosis of girls with idiopathic central precocious puberty (ICPP) and to analyze the relevance between MRI and traditional indicators. Methods. Selected 36 typical ICPP and 8 early ICPP, IGF-1, LH, FSH, E2, FT3, FT4, TSH at fast time, and the peaks of LH, and LH/FSH after the stimulation of $\mathrm{GnRH}$ were examined, all of the patients with CPP underwent MRI scans of the hypothalamic-pituitary area, the B-mode uItrasound of ovaries and uterus, and bone age $\mathrm{BA}, 30$ healthy prepuberty and age-matched girls as the control group. Results. Pituitary height and IGF-1 were significantly higher in both typical and early ICPP group than in control group $(P<.01)$, there were no significant difference between the typical and early ICPP; CA, (BACA), peak LH, and LH/FSH, E2 in typical ICPP group were significantly higher than those in the group of early ICPP, $P<.01$ Gonadotropin-releasing hormone analogue (GnRHa) showed significant effect in the treatment of early ICPP. Pituitary height showed a significant linear positive correlation with (BA-CA), IGF-1, LH, and LH/FSH, GnRHstimulated peak LH, and LH/FSH, E2, (resp., $r=0.45,0.65$, $0.54,0.45,0.58,0.57,0.54, P<.05-.01$ ). Elster grades of anterior pituitary had a significant positive correlation with Tanner stages $(r=0.54, P<.001)$. The children with ICPP which pituitary showed mild convexity and spherical on MRI accounted for $86.4 \%$. Conclusion. MRI and IGF- 1 are helpful in the early diagnosis of ICPP girls. MRI is closely related to the clinical manifestations and the functions of pituitarygonad axis.

\section{The Growth-Promoting Efficacy of Gonadotropin-Releasing Hormone Analogues on Idiopathic Central Precocious Puberty Girls}

Xie Liling, Yang Yu, Yang Li, Jiangwei Ke, Xian Wei, Haiying Zhou, Lirong Yu, Dongguang Zhang, and $Y_{i}$ Yuan

\section{Children's Hospital Of Jiangxi Province}

Objective. To observe the gonadotropin releasing hormone analogue (GnRH-a) on idiopathic central precocious puberty (ICPP) and projected adult height of girls growing influence. Methods. 200 patients with idiopathic central precocious puberty (ICPP) girls were treated with GnRHa treatment, 1 injection every 4 weeks, the first 3 times $100 \mu \mathrm{g} / \mathrm{kg}$, after $60 \sim 80 \mu \mathrm{g} / \mathrm{kg}$, for 12 to 36 months (average $21.1 \pm 7.43$ months) of treatment, bone age (BA), growth rate $(\mathrm{GV})$, predicted adult height $(\mathrm{PAH})$, and changes in sexual characteristics, height growth forecast value of the correlation with the course. The slower growth rate after treatment of bone age results, bone age (BA) growth of less than the age (CA) growth of 6 months after treatment, 12,24 , and 36 months BA/CA ratios were $0.86 \pm 0.39$, $0.61 \pm 0.28,0.48 \pm 0.32,0.22 \pm 0.26$, and $1.73 \pm 0.25$ before treatment were significantly different $(P<.01)$. The first year after treatment, the growth rate (GV1) was $6.4 \pm 1.25 \mathrm{CM}$, the second annual growth rate (GV2) for the $5.3 \pm 1.38 \mathrm{CM}$, the third annual growth rate (GV3) was $4.5 \pm 1.32 \mathrm{CM}$; predicted adult height $(\mathrm{PAH})$ before treatment was $151.26 \pm 4.63 \mathrm{CM}, 6$ months after treatment, the first year, second year, third year, respectively was 
$152.29 \pm 3.91,153.13 \pm 4.33,154.58 \pm 5.09,156.31 \pm 5.34$, compared with before treatment were significantly different $(P<.01)$, the third year of net PAH before treatment was $5.21 \pm 1.02 \mathrm{CM}$, close to the target height $156.39 \pm$ $4.24(P>.05)$. PAH growth in value and duration were significantly correlated $(r=0.75, P<.01), 3$ months after treatment serum levels of Estrogen and $\mathrm{LH}$, FSH peaks were reduced to pre-puberty, breast star back. Conclusion. GnRHa can effectively inhibit the growth of secondary sexual characteristics and bone age, to improve the prediction of adult height ICPP girls; when GnRHa treatment is longer, the growth of the value predicted adult height is greater; GnRHa treatment combined with growth hormone can more effectively improve the prediction adult height.

\section{Reproductive Axis, Uterus and Ovarian Volume and Menstrual Cycle after Long-Term Therapy with Gonadotropin-Releasing Hormone Agonist in Girls with Central Precocious Puberty}

\author{
Marta Snajderova, ${ }^{1}$ Daniela Zemkova, ${ }^{1}$ \\ and Leos Teslik ${ }^{2}$ \\ ${ }^{1}$ Department of Paediatrics, 2nd Medical School and \\ University Hospital-Motol, Prague, Czech Republic \\ ${ }^{2}$ Department of Gynaecology and Obstetrics, \\ 2nd Medical School and University Hospital-Motol, \\ Prague, Czech Republic
}

Gonadotropin-releasing hormone analogs (GnRHa) are standard of care for central precocious puberty (CPP). Only a few data on the development of girls with CPP after longterm therapy are available. We are interested in the regulation of reproductive axis and sexual development in girls with central precocious puberty after therapy. Thirty two girls with CPP ( $n=19$ idiopathic CPP; $n=13$ organic CPP), at the end of therapy aged $11.1 \pm 1.1$ years (mean \pm SD) (range 9.3-14.1), treated $3.1 \pm 1.3$ years (range 1.0-7.2 years) with D-Trp6-GnRH agonist were observed. Volumes of uterus, ovaries, and standard GnRH test (LHmax/FSHmax) were assessed at the end of therapy, 3, 12, 24, and 36 months thereafter (GnRH test only until menarche) and results were compared with previous time. Menstrual cycle was analysed 12, 24, and 36 months after therapy withdrawal. Similar trends in gonadotropin levels, ovarian, and uterus volume were found both in girls with idiopathic (ICPP) and organic (OCPP) central precocious puberty. LHmax/FSHmax at the end of therapy $0.3 \pm 0.3$ increased within 3 months to $1.2 \pm 0.7(P<.001)$; compared with previous time $)$ and to $3.0 \pm 3.2(P<.01) 12$ months after therapy. Ovarian volume $2.2 \pm 0.9 \mathrm{ccm}$ at the end of therapy increased within 3 months to $3.3 \pm 2.0 \mathrm{ccm}(P<.01)$ and at 12 months to $4.4 \pm 1.8 \mathrm{ccm}(P<.05)$. No significant changes were found 24 and 36 months since therapy withdrawal. Uterus volume $4.2 \pm 17 \mathrm{ccm}$ at the end of long-term GnRHa therapy increased within 3 months to $11.6 \pm 9.6 \mathrm{ccm}(P<.01)$, at
12 months to $21.1 \pm 9.4 \mathrm{ccm}(P<.01)$, at 24 months to $37.8 \pm 16.9 \mathrm{ccm}(P<.05)$, and 36 months after the end of therapy to $39.6 \pm 17.7 \mathrm{ccm}$ (ns). Menarche was reached at the mean age $12.7 \pm 1.2$ years (bone age $13.7 \pm 0.9$ years), $1.5 \pm 0.8$ years after the end of therapy, earlier in ICPP group $(1.4 \pm 0.6)$ years versus $1.7 \pm 1.1$ years in OCPP group; $(P<.01)$. Irregular menstrual cycle was detected in $11 \%$ of girls 36 months after therapy withdrawal, more frequently within OCPP group. Volumes of uterus and ovaries correspond with the rapid increase of gonadotropin levels during the first year of posttreatment period. In the post treatment period, development of uterus corresponds with the biological age. We conclude that suppression of pituitary-gonadal axis after depot long-term GnRHa therapy in girls with central precocious puberty is fully reversible. Incidence of irregular menses did not differ significantly compared with general population.

\section{Consecutive Gonadotropin Determinations in Urine by Immunochemiluminometric Assays during Gonadotropin-Releasing Hormone Analog Stimulation Testing in Children}

\author{
Zhuang-jian Xu, Xi-ping Wang, Ya-ping Ma, \\ Wen-jie Zhang, Xing-fu Pang, Qing Wang, \\ Shu-yan Xie, and Ting-ting Zhang \\ Department of Pediatrics, The 4th Affiliated Hospital of \\ Soochow University, Wuxi, 214062, Jiangsu Province, \\ China
}

Objective. To observe gonadotropin excretion in urine by immunochemiluminometric assays (ICMA) during GnRHa stimulation testing in order to provide urinary gonadotropin (UGn) basic data for studying diagnosis of HPGA. Methods. Twenty four GnRHa stimulation testing in children who suffered from disorders of growth or pubertal development were performed in hospital. Nine consecutive timed urine samples (the first was timed 1.5-hour urine, and the others were, resp., timed 1-hour) were collected, respectively, after injecting triptorelin in 24 tests. Overnight urine before injecting triptorelin and 3 consecutive overnight urine after injecting triptorelin were collected, respectively, in 16/24 tests. The LH, and FSH were assayed by ICMA, and urinary gonadotropin (UGn) concentrations were corrected by creatinine (Cr). Results. (1) The excreting phase for the peak urinary $\mathrm{LH}$ (ULH) among 9 consecutive timed urine sampels: The upper 95\% confidence limit of time for the first peak ULH and the highest peak ULH (the quantities of ULH and the ratio of ULH concentrations/Cr) were, respectively, $4 \mathrm{~h} \sim 6.2 \mathrm{~h}$ and $7.2 \mathrm{~h} \sim 9.5 \mathrm{~h}$. And the number (median) of peaks and the upper 95\% confidence limit were 3 peaks and 4 to 4.75 peaks, respectively. (2) The correlation coefficient between the peak UGn and serum peak gonadotropin: The correlation coefficient between serum peak LH (PLH) and the first peak quantities of ULH was 0.927, and between serum PLH and the the highest peak quantities of ULH 0.856, and between serum PLH and the first peak ratio of ULH concentrations/Cr 0.851 , 
and between serum PLH and the highest peak ratio of ULH concentrations/Cr 0.826 . The correlation coefficient between serum PLH/peak FSH (PFSH) and (the first peak quantities of ULH)/(quantities of UFSHs) was 0.845 (UFSHs was the quantities of urinary FSH in the same first peak ULH sample), and between serum PLH/PFSH and (the highest peak quantities of ULH)/(quantities of UFSHs) 0.878 , and between serum PLH/PFSH and (the first peak ratio of ULH concentrations/Cr)/(the ratio of UFSHs concentrations/Cr) 0.861 , and between serum PLH/PFSH and (the highest peak ratio of $\mathrm{ULH}$ concentrations/Cr)/(the ratio of UFSHs concentrations/Cr) 0.843. (3) Consecutive UGn determinations in overnight urine: The correlation coefficient between serum PLH and the ratio of ULH concentrations/Cr in overnight urine before injecting triptorelin was 0.482 , and for the ratio of ULH concentrations/Cr in first overnight urine after injecting triptorelin 0.797, for the one in second overnight urine 0.653 , for the one in third overnight urine 0.212. And there was the highest median for the ratio of ULH concentrations/Cr in the first overnight urine among all overnight urine samples, then that in the second overnight urine decreased to the level that was same as in overnight urine before injecting triptorelin $(P>.05)$. Conclusions. Our data suggest that there was good correlation between consecutive timed UGn and serum peak $\mathrm{Gn}$ determined by ICMA during GnRHa stimulation testing in children. The effectiveness of triptorelin on pituitary almost disappears in $48 \mathrm{~h}$ after injecting triptorelin in children.

\section{The Circulating Levels of NTproCNP in Girls with Idiopathic Central Precocious Puberty}

\section{Yanan Zhang, Yalei Pi, Xue Yan, and Huifeng Zhang}

\section{The Department of Pediatrics, the Second Hospital, Hebei Medical University, Shijiazhuang, 050000, China}

Background. The features of plasma NTproCNP and the relationship between NTproCNP, IGF-I and estrogen in girls with idiopathic central precocious puberty. Methods. A total of 16 cases of girls with ICPP were set as ICPP group, while the control group involved 16 of healthy girls with age matched. Physical detection indices such as height, body weight and bone age were determined. The bone age was measured with TW-method, through evaluating the ossification center of the left hand and wrist. Plasma NTproCNP and IGF-I were measured by ELISA, while estrogen (E2) were determined by chemoluminescence assay. Results. (1) Compared with the control group, plasma NTproCNP level $(18.92 \pm 8.62 \mathrm{pmol} / \mathrm{ml}$ versus $21.74 \pm 13.12 \mathrm{pmol} / \mathrm{ml}, z=-2.34), P<.05)$, serum IGF-I level $(522.41 \pm 228.33 \mathrm{ng} / \mathrm{ml}$ versus $867.50 \pm$ $318.15 \mathrm{ng} / \mathrm{ml},(t=-3.59, P<.05)$ and serum estrogen level $(19.00 \pm 4.5 \mathrm{pg} / \mathrm{ml}$ versus $37.19 \pm 16.08 \mathrm{pg} / \mathrm{ml}$, $z=-2.58, P<.05)$ in ICPP group were significantly higher. (2) In control group, plasma IGF-I level had significant positive correlation with both age and bone age $(r=0.65, P<.01 ; r=0.66, P<.01)$, whereas no such correlation existed in ICPP group. In both ICPP group and control group, plasma NTproCNP level had no correlation with neither age nor bone age; Moreover, plasma NTproCNP had no correlation with IGF-I; No correlation was found between NTproCNP, IGF-I and estrogen. Conclusion. NTproCNP may play a role in the acceleration process of longitudinal growth of bone in the girls with ICPP.

\section{Longitudinal Estimates of Pubertal Development in Girls Maturating at Different Ages}

\section{YanHong Li, HuaMei Ma, HongShan Chen, Zhe Su, YuFen Gu, and MinLian Du}

\section{The First Affiliated Hospital of Sun Yat-Sen University, GuangZhou 510080, China}

Objective. To investigate pubertal growth pattern in relation to the timing of onset of puberty in healthy Cantonese girls. Method. 320 normal Cantonese schoolgirls, recruited at age of $7.24 \pm 0.38$ years (6.25 to 8.83 years), were followed up annually for 9 years (from 1992 to 2001). Physical examinations included height, weight and pubertal stages. Children were subdivided into 4 groups according to the ages at the onset of puberty (B2): group 1: onset less than 9 years, group 2: onset between 9 to 10 years, group 3: onset between 10 to 11 years, group 4: onset later than 11 years. Results. 274 girls (85.6\%) had submitted menarche, 250 girls $(78.1 \%)$ had reached final adult height (FAH). Girls with earlier puberty reached menarche at earlier ages $(11.39 \pm 1.38$ years, $12.08 \pm 1.25$ years, $12.77 \pm 1.14$ years, $13.45 \pm 1.09$ years, $P<.001)$, but exhibited longer durations from $\mathrm{B} 2$ to peak height velocity (PHV) $(1.52 \pm 1.01$ years, $1.23 \pm 0.80$ years, $0.74 \pm 0.61$ years, $0.52 \pm 0.36$ years, $P<.001)$ and from menarche to FAH $(2.36 \pm 1.16$ years, $2.18 \pm 1.31$ years, $1.90 \pm 0.99$ years, $1.48 \pm 1.11$ years, $P=$ .014), however, intervals from PHV to menarche were similar to the late maturers $(2.10 \pm 1.25$ years, $1.83 \pm 1.18$ years, $1.82 \pm 1.06$ years, $1.99 \pm 0.94$ years, $P=.405)$. In girls with early puberty, height Z-score at B2, PHV $(\mathrm{cm})$ and the height attained from menarche to $\mathrm{FAH}$ were higher than those matured later: $(0.24 \pm 0.99,0.25 \pm 0.69,-0.28 \pm 0.82$, $-0.20 \pm 0.85, P=.003),(8.60 \pm 0.90 \mathrm{~cm}, 8.31 \pm 0.92 \mathrm{~cm}$, $8.12 \pm 1.12 \mathrm{~cm}, 7.60 \pm 0.97 \mathrm{~cm}, P<.001),(6.69 \pm 4.04 \mathrm{~cm}$, $6.07 \pm 4.00 \mathrm{~cm}, 5.24 \pm 3.82 \mathrm{~cm}, 4.04 \pm 3.41 \mathrm{~cm}, P=.029)$, but no differences of FAH were found between groups (158.58 \pm $6.44 \mathrm{~cm}, 158.09 \pm 5.73 \mathrm{~cm}, 158.59 \pm 6.05 \mathrm{~cm}, 159.24 \pm$ $5.93 \mathrm{~cm}, P=.857)$. Conclusions. Pubertal growth patterns depend on the timing of the onset of puberty in healthy Cantonese girls. Although menarche may occur earlier, girls matured at early age exhibit the compensatory pubertal development, comprising longer duration of puberty growth and higher peak height velocity, namely the increased total height attainment during puberty, and thus have unimpaired FAH. 


\section{Miscellaneous}

\section{Self-Esteem, Behavior, and Social Functioning in Girls with Turner Syndrome}

\author{
Mei-Chyn Chao, ${ }^{1,2}$ Cheng-Yu Liao, ${ }^{1}$ \\ and Sa-Chin Guan ${ }^{3}$ \\ ${ }^{1}$ Division of Genetics, Endocrinology and Metabolism, \\ Department of Pediatrics, Kaohsiung Medical University \\ Hospital, Kaohsiung, Taiwan \\ ${ }^{2}$ Department of Medical Genetics, College of Medicine, \\ Kaohsiung Medical University, Kaohsiung, Taiwan \\ ${ }^{3}$ Department of Psychology, Kaohsiung Medical University, \\ Kaohsiung, Taiwan
}

TS is a sex-chromosome disorder which is caused by the total or partial absence of one $\mathrm{X}$ chromosome in some or all cells. The principal features are short stature and dysfunctional gonads, resulting in a lack of sex hormones, incomplete pubertal development and impaired fertility. Previous studies from other countries indicated that girls with TS are at higher risk of having social and behavioral problems than the general population. Besides, parents of girls with TS have rated them as having significantly more behavioral problems and lower social competence than did parents in a normative sample, and girls with TS have rated themselves as having social problems, and have reported being dissatisfied with their physical appearance. The objective of this study is to describe self-esteem, behavior and social functioning in all known girls with Turner syndrome (TS), 7-16 years of age. Participants are recruited from the Department of Pediatrics of Chung-Ho Memorial Hospital, Kaohsiung Medical University. Forty girls with TS (range, 7-16 years) participate in a cross-sectional study using the Rosenberg Self-esteem Scale (RSE) and the Child Behavior Checklist (CBCL). Students $t$-test are used to compare the RSE scores with normative data, and the Mann-Whitney test are used to compare CBCL scores with normative data. Spearmans rank correlation coefficient (rs) are used to examine the relationship between age, RSE scores and CBCL scores. Descriptive statistics are given as means and standard deviations (SD) for the RSE, and as raw scores for the CBCL. The results from this study may support previous studies from other countries that girls with TS are at higher risk of having social and behavioral problems than the general population. These may also help us understanding the selfesteem in TS and these problems should be considered when providing further clinical care.

\section{Endocrine Dysfunction after Bone Marrow Transplantation during Childhood and Adolescence}

Hye-Young Jin, Jin-Ho Choi, Ho-Joon Im, Jong-Jin Seo, and Han-Wook Yoo

Department of Pediatrics, Asan Medical Center Children's Hospital, University of Ulsan College of Medicine, Seoul, Korea
Purpose. Several complications can occur in patients who received bone marrow transplantation (BMT) during childhood and adolescence. This study aims to investigate endocrine dysfunctions after BMT so that better care can be provided to care for long-term survivors of BMT. Methods. One hundred patients (61 males, 39 females) were included in this study. Clinical parameters such as initial diagnosis, age at BMT, conditioning regimen, presence of graft-versushost disease (GVHD), growth pattern, thyroid function, and pubertal status were retrospectively reviewed to evaluate risk factors associated with endocrine dysfunction. Results. Height standard deviation score (SDS) at BMT, after 1 year of BMT, and at the last visit were $0.08 \pm 1.04$, $-0.09 \pm 1.02$, and $-0.27 \pm 1.18$, respectively, $(P=.001)$. Height SDS significantly decreased in patients who received total body irradiation (TBI) $(P=.017)$. One of the patients who received TBI demonstrated growth hormone deficiency. Thirty (31.9\%) of 94 patients had compensated hypothyroidism. Incidence of compensated hypothyroidism was higher among those who had GVHD (odds ratio 2.82, $P=.025)$. Of the 32 patients (17 males, 15 females) who were over 14 years in male and 13 years in female at the last visit, 16 (3 males, 13 females) had increased luteinizing hormone ( $\mathrm{LH})$ or follicle-stimulating hormone (FSH). Abnormal elevation of LH or FSH was more common in females (odds ratio 30.3, $P=.001$ ). Conclusion. The most common endocrine dysfunction was ovarian insufficiency. Regular checkup for endocrine function needs to be required due to high incidence of endocrine dysfunction in patients with BMT.

\section{Differences in the Anthropometric and Endocrine Characteristics of Children Conceived after the Transfer of a Fresh or Thawed Embryo Compared to Naturally Conceived Controls}

\author{
Mark Green, ${ }^{1}$ Frances Mouat, ${ }^{1}$ Harriet Miles, ${ }^{1}$ \\ Sarah Hopkins, ${ }^{1}$ Paul Hofman, ${ }^{1}$ John Peek, ${ }^{2}$ \\ and Wayne Cutfield ${ }^{1}$

\section{${ }^{1}$ The Liggins Institute, University of Auckland, Auckland, New Zealand \\ ${ }^{2}$ Fertility Associates, Auckland, New Zealand}

Objectives. To determine whether anthropometric or endocrine characteristics differ in IVF children; conceived via fresh or thawed embryo transfer, compared to naturally conceived controls. Methods. Healthy prepubertal children (3.5 to 11 years) born at term after a singleton pregnancy were evaluated. Naturally conceived controls $(\mathrm{CON} ; n=$ 94) were compared to IVF children conceived via the transfer of a fresh (IVFF; $n=72$ ) or thawed (IVFT; $n=43$ ) embryo. Anthropometric characteristics and body composition (DEXA) were assessed, as well as fasting metabolic (glucose and insulin) and endocrine (IGF-I,-II and BP3) hormones measured. All anthropometric data were standardised (SD scores) and adjusted for parental size. 
Data were analysed by linear regression and mixed model ANOVA with appropriate covariates. All data are reported as means \pm SEM. Results. IVFF children were lighter at birth $(P<.001)$ than CON and IVFT. After adjustment for parental size, IVFF children were taller $(P<.05)$ than both CON and IVFT contemporaries. Although no differences in corrected BMI were evident, IVFT children had lower $(P<.05)$ percent body fat compared to CON but not IVFF children. All hormones were positively $(P<$ $.05)$ correlated with height and percent fat. No differences were found in glucose or insulin concentrations. IGF-I was higher $(P<.05)$ in IVFF than the other two groups. In contrast, IGF-II was higher $(P<.05)$ and IGFBP3 lower $(P<.01)$ in the IVFT children compared to CON, and CON and IVFF children, respectively. Conclusion. Subtle differences exist between IVFF (taller with higher IGF-I concentrations) and IVFT (leaner with higher IGF-II and lower IGFBP3 concentrations) children, in addition to those identified between IVF and CON children. The divergent anthropometric and biochemical characteristics of the two IVF groups may be explained by a combination of differences in embryo selection, in vitro processing, or the transfer of an embryo to a uterus exposed to ovarian IVF superstimulation regimes. However, the underlying mechanisms involved are yet to be elucidated and are the focus of continued research.

\section{Polyuria in an Infant Caused by Primary Polydipsia}

\section{Muhammad Faizi, Bagus Samsu Tri Nugroho, Achmad Yuniari Heryana, and Netty Harjantien}

\section{Division of Endocrinology, Department of Child Health, Faculty of Medicine, University of Airlangga/Dr. Soetomo Hospital, Surabaya}

Background. Excessive and persistent fluid intake or commonly called primary polydipsia is one of causes of polyuria in children, beside nephrogenic diabetes, diabetes mellitus and diabetes insipidus. Undiagnosed and untreated polyuria can cause suffering and result in failure to thrive, hyperosmolar state, severe dehydration, irreversible brain damage and death. Objective. To report a case of polyuria in an infant caused by primary polydipsia. Case Report. A 2-month-old boy was referred with suspicion of diabetes insipidus. $\mathrm{He}$ came with frequent urination for 7 days, spent 4 dozen of cloth diapers a day. He got frequent breast feeding. His mother was told by a previous health caregiver to feed the baby frequently because of his prematurity and low birth weight. Urine production was $10.1 \mathrm{cc} / \mathrm{kg} / \mathrm{hour}$ (polyuria $>4 \mathrm{cc} / \mathrm{kg} /$ hour). Laboratory tests revealed random blood glucose was $85 \mathrm{mg} / \mathrm{dL}$, creatinine serum was $0.4 \mathrm{mg} / \mathrm{dL}$, BUN was $9 \mathrm{mg} / \mathrm{dL}$, urine specific gravity (SG) was $1.005(\mathrm{~N}: 1.010)$ and plasma osmolarity (POsm) was 277.9 mOsm (N: 282-295 mOsm). Results of head CTscan and kidney ultrasound were normal. Water depriviation test was done to distinguish DI and primary polydipsia.
After the test, urine production decreased to $3.27 \mathrm{cc} / \mathrm{kg} / \mathrm{hour}$, POsm $295.79 \mathrm{mOsm} / \mathrm{L}$ (<300 mOsmL), SG 1.010. He had primary polydipsia. Polyuria was improved by feeding reschedule according to his daily requirement. Conclusions. Inappropriate feeding behavior (excessive feeding) may cause polyuria. Water deprivation test can be used to differentiate polyuria caused by primary polydipsia or diabetes insipidus.

\section{Mandibuloacral Dysplasia with Type A Partial Lipodystrophy: A Case Report}

\author{
Ganesh Jevalikar, Ambrish Mithal, \\ and Abhimanyu Garg
}

\section{Department of Endocrinology, Indraprastha Apollo Hospital, New Delhi, India}

Background. Mandibuloacral dysplasia is a rare autosomal recessive disorder associated with lipodystrophy which is an uncommon cause of oligomenorrhea. Case Report. A nineteen year old girl presented with irregular menses and excessive scalp hair loss since menarche which was at 11 years of age. She had a mottled cutaneous pigmentation. She had mild hirsutism and acne. She was receiving metformin and oral contraceptives for above complaints. She had disproportionate fat distribution in the form of presence of fat in truncal region and lack of fat from the extremities. There was no intake of androgenic medications. Family history was unremarkable. On examination her weight was $47 \mathrm{~kg}$ and height was $149.7 \mathrm{~cm}$. Her blood pressure was $130 / 86 \mathrm{~mm}$ of Hg. She had muscular appearance of upper limbs with paucity of fat and excess truncal fat. Her fingers and toes were short and thin with bulging of terminal phalanges. She had small pointed nose, small mandible and narrow shoulders. There was mottled pigmentation of neck, trunk and elbow flexures. Acanthosis was absent. Thyroid was normally palpable. Systemic examination was unremarkable. Her serum testosterone was $0.68 \mathrm{nmol} / \mathrm{L}$. Thyroid profile, serum prolactin and 17-hydroxyprogesterone levels were normal. She had fasting hyperinsulinemia $(243.6 \mathrm{pmol} / \mathrm{L})$ and impaired glucose tolerance ( 2 hours post glucose load blood glucose $-7.7 \mathrm{mmol} / \mathrm{L}$ ). Her fasting lipid profile showed total cholesterol of $4.88 \mathrm{mmol} / \mathrm{L}$, LDL cholesterol of $2.86 \mathrm{mmol} / \mathrm{L}$ and triglyceride of $2 \mathrm{mmol} / \mathrm{L}$. X-ray of the hands showed osteolysis of terminal phalanges and Xray of the shoulders revealed destruction of the clavicle. The echocardiogram was normal. She was managed with dietary advice, lifestyle modification. Oral contraceptives and metformin were continued. Discussion. Mandibuloacral dysplasia is a rare autosomal recessive disorder caused by mutations in LMNA gene and zinc metalloproteinase (ZMPSTE24) gene. Mutations result in apoptosis and premature cell death of adipocytes. It is associated with two distinct patterns of lipodystrophy. Type A involves the loss of subcutaneous fat from the arms and legs but normal or excess deposition of fat in the face and neck, and type $\mathrm{B}$ is characterized by a more generalized loss of subcutaneous fat. Some of the patients may not have mutations 
in both of the above genes suggesting presence of other loci.

\section{A Case of Immune-Dysregulation, Polyendocrinopathy, Enteropathy, X-Linked (IPEX) Syndrome Presenting as Pure Red Cell Aplasia and Posterior Reversible Encephalopathy Syndrome}

\author{
Hye-Young Jin, ${ }^{1}$ Keun Wook Bae, ${ }^{1}$ Jin-Ho Choi, ${ }^{1}$ \\ Jong Jin Seo, ${ }^{1}$ Young Seo Park, ${ }^{1}$ Gu-Hwan Kim, ${ }^{2}$ \\ and Han Wook Yoo ${ }^{1,2}$ \\ ${ }^{1}$ Department of Pediatrics, Asan Medical Center Children's
Hospital, University of Ulsan College of Medicine, Seoul,
Korea
${ }^{2}$ Medical Genetics Clinics and Laboratory,
Asan Medical Center, Seoul, Korea
}

Background. The syndrome of immune-dysregulation, polyendocrinopathy, enteropathy, X-linked (IPEX) is a rare $\mathrm{X}$-linked recessive disorder, usually manifesting as multisystemic autoimmune disorders during infancy. Most patients have been reported to die within the first 2 years of life due to severe infection or failure to thrive. We describe here a patient with IPEX syndrome with a novel splicing mutation of the FOXP3 gene. Case. The patient was a male child, born healthy at $39+2$ weeks of gestation. At 11 months of age, he was diagnosed with type 1 diabetes mellitus (T1DM). At 39 months of age, he developed acute anemia with a plasma hemoglobin concentration of $2.3 \mathrm{mg} / \mathrm{dL}$. Direct and indirect Coombs' test results were $3+$ and $1+$, respectively, however, hemolysis was not obvious on a peripheral blood smear. Bone marrow biopsy showed adequate erythropoiesis. He was diagnosed with pure red cell aplasia (PRCA) and started on steroid treatment with $2 \mathrm{mg} / \mathrm{kg} /$ day of prednisolone. A renal biopsy was performed for evaluation of incidentally detected albuminuria on routine urinalysis, and confirmed the diagnosis of membranous glomerulonephritis (MGN). $\mathrm{He}$ was also treated for severe bacterial pneumonia at the age of 7 years and for bacterial meningitis at the age of 11 years. DNA analysis of the FOXP3 gene identified a novel splicing mutation (c.201 + $1 \mathrm{G}>\mathrm{A}$ ). The same mutation was found in his mother's FOXP3 genes as a heterozygote. Four months after recovery from meningitis, he developed posterior reversible encephalopathy syndrome (PRES) with severe hypertension after taking a vaccine against pandemic influenza A H1N1 virus. Following antihypertensive therapy, his neurological findings returned to normal, and he was discharged without any symptoms after two weeks. He has been treated with a very low dose of prednisolone and intensive insulin regimen. Conclusion. This patient presented with unusual clinical characteristics, including pure red cell aplasia, membranous glomerulonephropathy, and posterior reversible encephalopathy syndrome associated with sudden severe hypertension after vaccination. Although IPEX syndrome is usually a disease of infancy, it should not be ruled out solely on the basis of age. Its presentations are so variable that IPEX should be suspected in a male child with one or more autoimmune disorders and severe infections.

\section{Evaluation of Growth and Gonadal Maturity in Children and Adolescents on Treatment for Lupus Nephritis Class III/IV}

\section{Sophy Korula, Indira Agarwal, and Anna Simon \\ Department of Child Health, Christian Medical College, Vellore}

Objective. To determine the risk of growth retardation and gonadal toxicity in children treated for Class III/IV Lupus nephritis. Design And Setting. Concurrent cohort study. Methodology. 22 Children with SLE nephritis class III/IV were enrolled into the study over an 18 month period from April 2008-October 2009. 14 received cyclophosphamide (CYC) and 8 received mycophenolate (MMF). All received steroids and hydroxychloroquine. All of them had the routine investigations at followup including Anti-Ds Dna, Se complement and Se Creatinine. Growth delay was assessed by growth velocity measurement and correlation with the midparental height centile. Se vit D level, Se calcium, Se phosphorus were also checked for those with growth delay. Gonadal maturation was assessed by SMR staging. Gonadal toxicity was evaluated based on presence of amenorrhea, elevated FSH, LH levels and decreased ovarian size/endometrial thickness on ultrasound. Results. 22 children were included ( 3 were males). Mean age at study was 13.6 years (range 8-19 years). Mean duration of therapy was $35.07 \mathrm{mns}$ (range 1162) in CYC group, 16.06 mns (range 6-33) in MMF group. Average cumulative dose of CYC was $11.4 \mathrm{gm} / \mathrm{m}^{2}$. ${ }^{*} 7 / 22$ $(31.8 \%)$ had growth delay. Of these 3 of them had high disease activity and 4 had low Se vit D levels. The mean Se vit $\mathrm{D}$ level was $21.9 \mathrm{ng} / \mathrm{ml}$ in those with growth delay. ${ }^{*}$ Of the 19 girls, 11 attained menarche and had SMR breast stage 3. Three of them had sustained amenorrhea ( $>4$ months). Of these 3 , severe disease was noted in 2. One was on MMF and 2 on CYC $\left(P=.99\right.$, mean dose of $\left.10.6 \mathrm{gm} / \mathrm{m}^{2}\right)$. Among the remaining 8 girls- 5 were prepubertal, 3 were $\geq 12$ years. Of these 3 girls- 2 had developed thelarche (breast stage 2 ) and in 1 there was family history of maturational delay. There were 3 boys in this study, 2 were in early puberty based on SMR. No child had elevated FSH, LH levels. Conclusions. Growth delay was a cause of concern in children with lupus nephritis class III/IV. This was probably caused by a combination of the severe disease activity and vit D deficiency. Sustained amenorrhea was seen in $3 / 11$ girls (27\%) and was probably related to the disease severity rather than drug toxicity. None of the children had hormonal profile to qualify for premature gonadal failure during the period of study. A follow up of these patients will be necessary to confirm the same. 


\section{Glucose Homeostasis and \\ Body Composition in Survivors of Childhood Acute Lymphoblastic Leukemia}

\author{
Alisa Kulpiya, ${ }^{1}$ Preamrudee Poomthavorn, ${ }^{1}$ \\ Pat Mahachoklertwattana, ${ }^{1}$ Suradej Hongeng, ${ }^{1}$ \\ Samart Pakakasama, ${ }^{1}$ Chanika Sritara, ${ }^{2}$ \\ and Patcharin Khlairit ${ }^{1}$ \\ ${ }^{1}$ Department of Pediatrics, \\ ${ }^{2}$ Department of Radiology, Faculty of Medicine, \\ Ramathibodi Hospital, Mahidol University, Bangkok10400, \\ Thailand
}

Background. Improvement in the treatment of childhood acute lymphoblastic leukemia (ALL) has led to a growing number of survivors. ALL survivors are at increased risks of obesity, abnormal body composition and insulin resistance as a result of the treatment modalities and hormonal disorders. We aimed to study glucose homeostasis and body composition in survivors of childhood ALL. Methods. Childhood ALL survivors who had been in remission for at least 1 year were enrolled in the study. They were excluded if they had syndromic obesity or medications affecting glucose metabolism. Auxological data and history of ALL treatment were obtained. Oral glucose tolerance test (OGTT) with plasma glucose and insulin levels measurement was undertaken in all patients. Insulin sensitivity indices derived from the obtained glucose and insulin levels were calculated. All patients underwent dual energy X-ray absorptiometry (DXA) for body composition analysis. Data are presented in mean (SD). Results. There were 34 patients, aged 15.6 (3.8) years enrolled in the study. There were $21(61.8 \%)$ males. Twenty-nine $(85.3 \%)$ patients had already entered puberty. Age at diagnosis of ALL was 5.9 (3.1) years. All of them were treated with standard ALL chemotherapy protocol with 9 of them $(26.5 \%)$ had prophylactic cranial irradiation. They had been in remission for 6.6 (3.0) years. Weight and height Z-scores at enrollment were 0.9 (1.8) and $0(0.8)$, respectively. BMI was $21.0(4.2) \mathrm{kg} / \mathrm{m}^{2}$ and BMI $\mathrm{Z}$-score was 0.0 (1.3). Waist circumference (WC) and WC percentile were $74.3(11.4) \mathrm{cm}$ and 46.3 (33.9), respectively. Percentages of body fat and lean mass were 30.2 (11.0) and 66.3 (10.4), respectively. Of 34 patients, 15 (44\%) had an abnormal OGTT result with 13 of them had hyperinsulinemia, that is, insulin resistance and 2 had impaired glucose tolerance. Homeostatic model assessment of insulin resistance (HOMA-IR) and whole body insulin sensitivity index (WBISI) were 1.6 (1.2) and 7.0 (4.9), respectively. In comparison with patients having normal OGTT results, patients with abnormal OGTT results had greater HOMAIR (2.2 (1.3) versus $1.1(0.7) ; P=.007)$ and less WBISI (4.0 (1.6) versus $9.4(5.3) ; P<.001)$. However, percentage of body fat was not different between patients with abnormal and normal OGTT results $(32.6$ (10.2) versus 28.4 (11.6)\%; $P=.276)$. Conclusions. Childhood ALL survivors have an increased risk of insulin resistance despite being non-obese. This finding may be partly caused by relatively increased fat mass found in this group of patients.

\section{Impact of Intravenous Immunoglobulins on the Endocrinologic Milieu in Kawasaki Disease}

\author{
Kenichi Miyako, Naohiro Suga, Tomoko Hidaka, \\ Hiroshi Koga, and Noboru Takahashi
}

Beppu Medical Center

Kawasaki disease is a systemic vasculitis syndrome that usually develops in very young children. Options for treatment include aspirin and intravenous immunoglobulins. Immunoglobulin preparations are plasma derivatives from which blood-borne pathogens have been safely eliminated. However, remnants of endocrine substances have not been closely studied. We have found relatively high levels of fT3 and E2 in several lots of immunoglobulin preparations. To evaluate the impact of intravenous immunoglobulin preparations, we examined changes in serum hormone concentrations after a $2-\mathrm{g} / \mathrm{kg} / 24$ hours dose in children with Kawasaki disease ( $n=4$; age, 8 months -2 years 8 months). Changes in mean concentrations were as follows: fT3 $1.96 \rightarrow 2.56 \mathrm{pg} / \mathrm{ml}(P=.08)$, fT4 $1.21 \rightarrow 1.31 \mathrm{ng} / \mathrm{dl}$ $(P=.67)$, TSH $1.88 \rightarrow 3.99 \mu \mathrm{IU} / \mathrm{ml}(P=.01), \mathrm{LH}$ $0.10 \rightarrow 0.12 \mathrm{mIU} / \mathrm{ml}(P=.39), \mathrm{FSH} 0.55 \rightarrow 0.57 \mathrm{mIU} / \mathrm{ml}$ $(P=.86)$, and E2 $42.0 \rightarrow 16.5 \mathrm{pg} / \mathrm{ml}(P=.19)$. Analyses after adjustment by hemoglobin or creatinine levels showed similar trends. Changes in thyroid function test results in an infant treated with only aspirin were as follows: fT3 $2.7 \rightarrow 3.7 \mathrm{pg} / \mathrm{ml}$, fT4 $1.03 \rightarrow 1.28 \mathrm{ng} / \mathrm{dl}$, and TSH $1.39 \rightarrow$ $2.38 \mu \mathrm{IU} / \mathrm{ml}$. No change in any variable of thyroid function test was clinically significant. Because the infant treated with aspirin showed similar trends, changes in serum thyroid hormone levels may reflect the recovery phase of euthyroid sick syndrome. Although there was no elevation of E2 level, stimulation of the gonadotropin axis should be considered in patients with other diseases who repeatedly receive intravenous immunoglobulins. We are continuing our studies in additional patients.

\section{Report of Two Cases of ABCC8 Gene Mutation with Opposing Phenotypes: Neonatal Diabetes and Congenital Hyperinsulinism}

\section{Hooi Leng Ooi and Loo Ling Wu \\ Paediatric Endocrinology Unit, Department of Paediatrics, University Kebangsaan Malaysia Medical Centre, Jalan Yaacob Latif, Bandar Tun Razak, 56000 Cheras, Kuala Lumpur, Malaysia}

Introduction. KATP channel in the pancreatic b-cell is an important gateway to regulation of glucose-dependent insulin secretion. Activating mutation of KCNJ11 or ABCC8 genes which encode Kir6.2 and SUR1 subunit, respectively, in the KATP channel results in severe inhibition of insulin secretion manifesting as neonatal diabetes whereas loss of function mutations in the same genes can cause oversecretion of insulin and results in congenital hyperinsulinism. We report two cases of neonatal diabetes and congenital 
hyperinsulinism due to opposite mutations involving the ABCC8 gene. Case 1. TXY, 12-month old girl was delivered at term with birth weight of $2.5 \mathrm{~kg}$. She presented with severe diabetic ketoacidosis at five weeks of life. There were three episodes of seizures documented at presentation. Investigations showed negative pancreatic autoantibodies and normal pancreas on ultrasound. Genetic study revealed de novo heterogyzous activating missense mutation, Q211K, in exon 5 of the ABCC 8 gene. She was weaned off insulin and switched to glibenclamide at 8.5 months old. Currently she required glibenclamide $1.1 \mathrm{mg} / \mathrm{kg} /$ day with marked improvement in glycemic control and stable blood glucose profile. The latest HbAlc was $6.2 \%$ with no hypoglycaemia. She remained well and seizure free but had mild delay in gross motor development. Case 2. MA, 9-month old boy was born at term with birth weight of $4.1 \mathrm{~kg}$. He was the fifth progeny of a first cousin marriage. The elder two siblings died in early infancy due to hypoglycemia. MA had recalcitrant hypoglycaemia since birth but referred only at two months old. He did not response to diazoxide but maintained normoglycemia with glucagon infusion and subcutaneous octreotide injection. $\mathrm{He}$ underwent 95\% subtotal pancreatectomy at three months old however hypoglycaemia recurred two weeks after surgery. Parents refused further operation and patient continued on octreotide injection. He had delayed development with poor sucking reflex. Genetic study revealed a novel homozygous frameshift mutation A736fs in exon 16 of the ABCC8 gene resulting in a premature termination codon. Both parents are heterozygous carriers of the same mutation. Conclusion. The identification of ABCC 8 gene mutation in patients with neonatal diabetes and congenital hyperinsulinism not only confirmed the diagnosis but also had implications for treatment. For neonatal diabetes, oral sulphonylureas should be tried. Autosomal recessive congenital hyperinsulinism usually a diffuse disease and until further novel intervention available, subtotal pancreatectomy remains the mainstay of treatment.

\section{Growth Monitoring in Primary School Children}

\section{Voraluck Phatarakijnirund and Suttipong Wacharasindhu}

\section{Paediatric Endocrine Unit, Department of Paediatrics, Faculty of Medicine, King Chulalongkorn Memorial Hospital, Chulalongkorn University, Bangkok, Thailand}

Background. Growth monitoring is a part of child health supervision program in many countries worldwide. The aim of the program is to identify the growth disorder in an early stage of disease. In Thailand, there is no consensus guideline of growth monitoring program in school and the role of these has never been studied. Objective. To study the prevalence of growth disorder and to establish the role of growth monitoring for identification of growth disorder in primary school children. Method. A cross-sectional study of grade 3-6 children from 2 primary schools was conducted during July 2009-January 2010. The program consists of measuring of individual height and weight in all children. Short, and Tall statures were defined by either height below 3rd percentile or $>2$ major percentile below target height, and height above 97rd percentile or $>2$ major percentile above target height using Thai standard children growth charts, respectively. Obesity was diagnosed in children with ideal weight for height $>120 \%$. Children who met one of these criteria were completely examined to determine the definite cause of growth disorder. Result. Total number of subjects was 993 (523 boys and 470 girls. Prevalence of obesity, short and tall stature was $13.0 \%, 7.3 \%$, and $4.3 \%$, respectively. Among short stature group, $80.6 \%$ of children diagnosed according to their height below target height. Fifty children (80.6\%) in short stature group were completely examined. There was 1 new case of GHD, who had height above 3rd percentile but below target height, 1 child with known Turner syndrome and 1 diagnoses of malnutrition therefore the detect rate of growth disorder is $1: 281$. In tall stature group, $60.5 \%$ of children had height above 97rd percentile. Seven children $(17.9 \%)$ in tall stature group were completely examined and no new case of growth disorder was found. Within the obesity group, more than half of children $(60.8 \%)$ had mild obesity. Fifty-two children (47.3\%) in obesity group were examined, 11 of them had sign of insulin resistance and one, who was severe obesity, had hypertension. Conclusion. The results of the study have highlighted on the role of growth monitoring to detecting growth disorder in general children. The detection rate of short stature-related disorders is higher than previous report due to limit number of study population while the prevalence of obesity was similar to the previous report in Thailand over the past 10 years.

\section{Clinical Significance of Serum NT-proCNP, BAP, and OC in New Borns of Different Gestation Ages}

\author{
Pi Ya-lei, Zhang Hui-feng, Yan xue, \\ and Zhang Ya-nan
}

\section{Department of Pediatric, the Second Hospital of \\ Hebei Medical University, Shijiazhuang, Hebei 050000, China}

Objective. To determine the serum concentration of NTproCNP, BAP, OC in newnatals of different gestational ages. To investigate their clinical significance in evaluating bone growth. Methods. 80 newborns without congenital and metabolism disease, serious hypoxia or infections were chosed and divided into group $\mathrm{A}(26$ newborns, gestational age $\leq 32$ week, mean 29.2 week), B(28 newborns, 32 week $<$ gestational age $<37$ week, mean 34.1 week $)$ and $\mathrm{C}(26$ newborns, 37 week $\leq$ gestational age $<42$ week, mean 38.1 week). Body length and birth weight of all the newborns were determinded immediately after birth. Intravenous blood samples were collected within 24 hours after birth. Calcium and phosphor were measured by biochemistric method. Serum NT-proCNP, BAP, OC were measured by ELISA. Result. (1) There were no correlations between NTproCNP levels and birth weight, body length, BAP or OC levels. (2) There were negative correlations between BAP 
and gestational age, birth weight, body length. (3) There were positive correlations between OC and gestational age, birth weight, body length. Conclusion. (1) There is no correlation between NT-proCNP levels and fetus growth and development. (2) With the gestational age growth, the bone grows slower and the mineralization becomes stronger.

\section{A Full-Blown Case of Wolfram Syndrome in a Young Chinese Girl}

\author{
Grace Poon, ${ }^{1}$ Ivan Lo, ${ }^{2}$ Tony Tong, ${ }^{2}$ Pik To Cheung, ${ }^{1}$ \\ Anne Kwok, ${ }^{1}$ Joanna Tung, ${ }^{1}$ and Louis Low ${ }^{1}$ \\ ${ }^{1}$ Department of Paediatrics and Adolescent Medicine, \\ Queen Mary Hospital, The University of Hong Kong \\ ${ }^{2}$ Clinical Genetic Service, Department of Health, \\ The Government of the HKSAR
}

Introduction. Wolfram Syndrome is a rare autosomal recessive neurodegenerative disease, characterised by diabetes insipidus, diabetes mellitus, optic atrophy, deafness (DIDMOAD) and various other clinical manifestations including neurological symptoms, renal tract abnormalities, psychiatric manifestations, gastrointestinal dysmotility and gonadal disorders. Juvenile-onset diabetes mellitus and optic atrophy tend to present early at a median age of 6 years and 11 years, respectively. Cranial diabetes insipidus and sensorineural deafness often present in the second decade, whilst renal tract abnoramlaities present in the third decade, followed by neurological complications in the fourth decade. This progressive disorder often results in death before the age of 50 years and affected individuals lead an impaired quality of life due to neurological and urological complications. Case Report. A young Chinese girl was diagnosed to have non-autoimmune insulin-deficient diabetes mellitus at the age of 5 years. She complained of poor vision with gradually decreasing visual acuity since the age of 3 years and was diagnosed to have bilateral optic atrophy at the age of 9 years. MRI showed no significant lesions along the visual pathway. She was further diagnosed to have central diabetes insipidus and later with additional detrusor sphincter dyssynergia around her 10th birthday. MRI brain and pituitary gland were normal. Ultrasound showed bilateral distended pelvicalyceal system with dilated ureters despite treatment with DDAVP. She was put on Detrusitol and Imipramine with improvement. BAEP at 10 years showed bilateral click thresholds at $40 \mathrm{~dB}$, signifying mild hearing impairment bilaterally. She was also diagnosed to have agoraphobia without panic attack in the same year requiring psychotherapy and psychiatric followup. Genetic Analysis. PCR-based cycle sequencing of all 8 exons of the WFS1 gene was performed on peripheral blood leucocytederived DNA from the proband. Three different heterozygous mutations were detected: two missense mutations R456H and G736S and one nonsense mutation Q667X. Both G736S and Q667X mutations are well reported in patients with Wolfram syndrome and are likely to be the cause of the proband's disease. The $\mathrm{R} 456 \mathrm{H}$ mutation has been reported to be significantly associated with type 1 diabetes mellitus and may have a modifying effect. Parental genetic testing is underway. Conclusion. It is becoming apparent that not all diabetes presenting in childhood is type 1 and rare syndromic forms, such as Wolfram syndrome, have been identified in Hong Kong Chinese. Paediatricians should have a high index of suspicion of Wolfram syndrome when a child presents with juvenile-onset diabetes mellitus and optic atrophy.

\section{The Molecular Mechanism of Insulin Resistance in Intrauterine Growth Retardation Rats} Qianqi Liu, Lin Zhang, Xing Shi, Shining Ni, Wei Gu,
and Ziyang Zhu

Nanjing Children Hospital Affiliated to
Nanjing Medical University, Nanjing 210008, China

Objectives. (1) To investigate the expression of Insulin/ FoxO1/Pdx1/MafA genes and microRNA (miRNA) during pancreatic development in the intrauterine growth retardation (IUGR) rats. (2) To study the molecular mechanism of insulin resistance in IUGR rats. Methods. (1) Establishment of rat model: Female and male SD rats were copulated by $1: 3$, then the pregnant rats were divided into the following two groups: IUGR group and normal group. The former were treated by $50 \%$ calorie compared with normal group from gestational day 1 until term. (2) Collection of the samples: Pancreas of rat in embryonic day 14.5(E14.5), 19.5(E19.5) and new born were dissected under microscope, respectively. (3) Expression of Insulin/FoxO1/Pdx1/MafA genes: After abstraction of total RNA, the technique of RT-PCR was used to study the expression of insulin/FoxO1/Pdx1/MafA genes. (4) Expression of Pdx1/MafA protein: Western blotting was used to study the expression of Pdx1/MafA protein. (5) Expression of miRNA: Total RNA were harvested using TRIzol and RNeasy mini kit according to manufacturer's instructions. The samples were labeled using the miRCURY Hy3/Hy5 Power labeling kit and hybridized on the miRCURY LNA Array (v.11.0). (6) Confirmation of the results: The realtime PCR was used to confirm the miRNA array results. Results. (1) Insulin/FoxO1/Pdx1/MafA genes mRNA began to be expressed from E14.5, and the expression of Insulin and MafA were increased as the fetes grew while FoxO1 and Pdx1 were decreased. (2) The expressions of FoxO1 and MafA were lower than normal rats $(P<.05)$ while Insulin and Pdxl had no evident change. (3) Compared with the normal, the expressions of MafA protein were lower $(P<.05)$ while Pdx1 protein had no evident change in IUGR group. (4) In the normal group, comparing with embryonic day 14.5, 48 miRNAs of embryonic day 19.5 were up-regulated while 28 were down-regulated; 64 miRNAs of new born were up-regulated while 55 were down-regulated. Comparing with embryonic day 19.5, 40 miRNAs of new born were up-regulated while 52 were down-regulated. Comparing with the normal group, in the IUGR model, 10 were up-regulated while 26 were downregulated in embryonic day 14.5, 22 were up-regulated 
while 15 were down-regulated in embryonic day 19.5, 9 were up-regulated while 13 were down-regulated in new born. (5) The expression of miR-124let $-7 \mathrm{~d}^{*}$ mRNA were be chosen to confirm the miRNA array results: in normal group, miR-124 of the new born was increased comparing with embryonic day 14.5 and embryonic day 14.5 (FoldChange $>=1.5$ ), and let $-7 \mathrm{~d}^{*}$ was increased as the fetes grew (FoldChange $>=1.5$ ); in IUGR group, miR124 had no evident change comparing with the normal group $(0.67<$ FoldChange $<1.5)$, while let $-7 \mathrm{~d}^{*}$ was downregulated in embryonic day 19.5 and new born (FoldChange $<=0.67)$. The real-time PCR results were consistent with miRNA array results. Conclusions. (1) The expression of Insulin/FoxO1/Pdx1/MafA and miRNA in pancreas were effected by intrauterine growth retardation. (2) The molecular changes in IUGR rats pancrease could play an important role in adult insulin resistance which result from intrauterine growth retardation.

\section{Are Children Conceived with Maternal Clomiphene Physically or Metabolically Different?}

\section{Tim Savage, ${ }^{1}$ Mark Green, ${ }^{1}$ Paul Hofman, ${ }^{1}$ John Peek, ${ }^{1}$ and Wayne Cutfield ${ }^{2}$ \\ 1 The Liggins Institute, University of Auckland \\ ${ }^{2}$ Fertility Associates, Auckland}

Background. Clomiphene Citrate is widely used to aid fertility as it causes ovarian stimulation leading to increased oocyte number and differing oocyte morphology. Aims. To assess the growth and metabolism of children conceived with maternal clomiphene compared to naturally conceived children. Methods. Pre-pubertal children aged 3.5 to 10 years each had height, weight, pubertal assessment and body composition assessment by DEXA scan to determine fat mass percentage. Linear regression analysis compared their results to naturally conceived children of the same age, ethnicity and socioeconomic groups. Results. Interim analysis of preliminary data of 34 clomiphene conceived children compared to 94 naturally conceived children revealed the following results. Mean age of clomiphene conceived children and controls was 7.3 (S.E. 0.33) and 6.8 (SE 0.22) years, respectively. There was no significant difference in weight, BMI, fat percentage or height SDS when comparing the two groups. When children's height SDS was corrected for midparental height SDS, there was a trend of clomiphene conceived children being 0.4 SDS taller than naturally conceived children $(P=.07)$. Conclusion. Interim data analysis reveals a trend towards taller stature in clomiphene conceived children when corrected for genetic height. It appears that clomiphene treatment of mothers may be partially responsible for the increase in height seen in IVF offspring. This suggests that fertility medications may cause imprinting changes leading to taller stature in clomiphene conceived children.

\section{Physical Growth and Endocrine Profile of Thalassemic Children at an Indian Tertiary Care Hospital}

\author{
Rajni Sharma, Anju Seth, and Jagdish Chandra \\ Department of Pediatrics, Kalawati Saran Children's \\ Hospital and Lady Hardinge Medical College, New Delhi, \\ India
}

India has a large number of patients with transfusion dependent thalassemia major and there are few studies on physical growth and endocrinal profile of these children. We are presenting our experience of managing children with thalassemia major with a focus on growth and endocrine issues. This is a cross-sectional study of patients enrolled at the Thalassemia Day Care Centre at Kalawati Saran Children Hospital, Lady Hardinge Medical College, New Delhi. We evaluated the growth, pubertal status and endocrinal profile of patients aged 10-18-years $(n=58)$. Clinical and laboratory parameters included weight, height, sexual maturity ratings, general and systemic examination. Laboratory parameters were average pretransfusion hemoglobin, periodic serum ferritin and tests for viral markers HIV, Hepatitis B and C, liver function tests, blood sugar (fasting and post-prandial), calcium, phosphate and alkaline phosphatise (PTH in cases with hypocalcemia), thyroid profile, bone age and gonadotropins with gonadal hormones in patients with delayed puberty. $\mathrm{Z}$ scores for weight, height, body mass index were calculated using WHO reference data. Pretransfusion haemoglobin was well maintained (mean 9.2 g/dl 95\% CI 9.06-9.36) but the mean serum ferritin levels were approximately 3 times the desired value despite the patients being on regular chelation with deferiprone $(72 \%)$ or deferasirox $(25 \%)$. $86.2 \%$ of the patients had short stature. Delayed puberty or pubertal arrest was seen in $82.3 \%(42 / 51)$ of patients at or beyond the age of normal puberty. Diabetes mellitus/impaired glucose tolerance, hypothyroidism and hypoparathyroidism was seen in $6.8 \%, 8.6 \%$ and $12 \%$ patients, respectively. In conclusion, there is a high prevalence of growth and endocrine abnormalities in Indian children with thalassemia major.

\section{Thyroid Hormone Profile in Children with Sepsis and Its Correlation with Pelod Score as a Prognostic Parameter in Sepsis}

\author{
Agung Tanurahardjaa, Antonius Pudjiadib, \\ Risma Kaban, Husein Alatas, Pramita Gayatri, \\ and Aman Pulunganc \\ Department of Child Health, \\ Dr Cipto Mangunkusumo Hospital, Jakarta, Indonesia
}

Background. Thyroid hormones dysfunction can be found in sepsis, known as euthyroid sick syndrome or nonthyroidal illness. Aims. To evaluate the thyroid hormones dysfunction profile in children with sepsis and its correlation with 
PELOD score, outcome and PCT. This study also aimed to know the correlation between PELOD score and outcome. Methods. This is a cross sectional design. Subjects were children age 1-18 year old with sepsis admitted in PICU. PELOD score and blood examinations were taken once during the first 24 hours of PICU admission. Results. Thirty subjects were included. Median of T3, free T4 and TSH was $45(17-133) \mathrm{ng} / \mathrm{dL}, 0.81(0.3-1.57) \mathrm{ng} / \mathrm{dL}$ and 1.36 (0.05-7.78) $\mu \mathrm{IU} / \mathrm{L}$, respectively. T3, free T4 and TSH was decreased in $97 \%, 50 \%$ and $40 \%$ subject. There were no correlation between decreased thyroid hormone and PELOD score $(P=.218)$, outcome $(P=.55)$ and PCT $(P=$ $.694)$. The risk of death increased by 15 times among the subject with PELOD score $>20$ compare to PELOD score $<20(P=.012 ;$ OR $=15(95 \%$ CI $=1.535-146.545))$. Conclusions. Thyroid hormones were decreased in septic children with the majority of low T3. PELOD score can be used as a prognostic parameter for septic children at PICU.

\section{Kabuki Syndrome: One Case Report}

\section{Xuejing Yang and Feng Xiong \\ Children's Hospital of Chongqing Medical University}

Kabuki syndrome (KS) is also known as Kabuki makeup syndrome, Niikawa-Kuroki syndrome. The exact cause of the syndrome and the pathogenesis is still unknown. Attractive hypothesis is that $\mathrm{KS}$ belong to micro-deletion syndrome. The diagnosis based on the following five cardinal manifestations. These included a "peculiar face" (eversion of the lower lateral eyelid, arched eyebrows with the lateral one-third dispersed or sparse, depressed nasal tip, prominent ears and abnormal tooth morphology) in 100\% of their patients, skeletal anomalies (deformed spinal column with or without sagittal cleft vertebrae, and brachydactyly V) in $92 \%$ of their patients, mild to moderate mental retardation in $92 \%$ of their patients, dermatoglyphic abnormalities (fingertip pads, absence of digital triradius $\mathrm{c}$ and/or $\mathrm{d}$, and increased digital ulnar loop and hypothenar loop patterns) in $93 \%$ of their patients and growth deficiency in $83 \%$ of their patients (Postnatal growth retardation, but even more significantly with age. Complete or partial deficiency of growth hormone had ever reported). There have also been a number of less frequent findings reported in Kabuki syndrome, including visceral abnormalities, premature breast development in females, and susceptibility to frequent infections, Diaphragm abnormality, deaf. A boy, 4 years old, product of nonconsanguineous marriage, presented with retardation in growth and mental. He had facial dysmorphism with high arched eyebrows sparse laterally, long palpebral fissures with everted lower eyelids, blue sclera and long eyelash. His nose was broad with depressed tip, and ears were prominent and protruding, transverse palm-ar creases, persistent fetal finger pads. Auxiliary examinations shows the patient has moderate mental retardation, growth hormone deficiency, Atrial septal defect, moderate hearing loss. Karyotype done on peripheral lymphocytes was normal. He was diagnosed as Kabuki make up syndrome due to clinical findings. The patient was treated successfully with an ASD repair, and subcutaneous injections of growth hormone $0.1 \mathrm{U} / \mathrm{Kg} /$ day. The patient has grown $4 \mathrm{~cm}$ after 5 months. Because Kabuki syndrome is not associated with severe medical complications, it is presumed that the prognosis for survival into adulthood is good.

\section{Research on Body Proportions Reference Charts of 0-18 Years Children and Adolescents in China}

\section{Zhang Yaqin and Li Hui}

\section{Department of Growth and Development, Capital Institute of Pediatrics, Beijing 100020, China}

Objective. To know the status of body proportions of $0-18$ years Chinese children and adolescents in 2005, analyze secular changes of body proportions during 1985-2005, and obtain the reference charts of body proportions to provide reference data for doctors and researchers in related fields. Methods. Subjects were healthy han nationality children and adolescents in nine provinces (cities) from two national representative cross-sectional surveys in " 2005 ". The evaluation index of body proportions was the ratio of sitting height and leg length (SH/LL). Sitting height and height were obtained by measuring and Leg length was obtained by subtracting sitting height from height. Reference charts of SH/LL were formulated by LMS methods. Results. (1) SH/LL was decreased gradually with age, for example, SH/LL of newborn infants, 1 years, 3 years, 6 years children was, respectively, $2.0,1.68,1.43,1.25$ in both boys and girls. It reached the lowest at 12-13 years in boys and 1011 years in girls (the minimum of boys and girls was, respectively, 1.11, 1.13). Then $\mathrm{SH} / \mathrm{LL}$ increased slightly and finally unchanged after 17 years, the final SH/LL of boys and girls was, respectively, 1.16, 1.18. (2) Before 11 years, there was not gender difference in SH/LL, and after that SH/LL of girls was higher slightly than boys' SH/LL. The gender difference became largest in 13 years (difference value was 0.04$)$. SH/LL of urban and rural children was similar before 7 years, after that SH/LL of rural children was higher slightly than urban children (maximum difference value was 0.02), while the final SH/LL wasnot urban-rural difference. The SH/LL charts were almost coincident in different areas. (3) The final SH/LL was not changes during 1985-2005. While the age that reached the lowest point was advanced 1 year in 2005. Conclusions. SH/LL regularly changed with age in 0-18 years Chinese children and adolescents. There wasnot gender difference in prepubertal children, and SH/LL of girls was higher slightly than boys' SH/LL after postpubertal. There were not urban-rural difference, regional difference and secular changes in SH/LL in China. Reference charts of SH/LL in this study were suitable as evaluated standards of body proportions of Chinese children and adolescents. 


\section{Relationship between Genetic Polymorphisms Involved in Folate/Homocysteine Metabolic Pathway and Risk of Down Syndrome}

\section{Juan Ye and XP Luo}

\author{
Department of pediatrics, Tongji Hospital, \\ Tongji Medical College, Huazhong University of \\ Science and Technology, Wuhan, Hubei 430030, China
}

Objective. to investigate the possible links between six common genetic polymorphisms involved in folate/homocysteine metabolic pathway and risk of Down syndrome in Han nationality. Methods. 34 mothers who had ever gestated or given birth to children with Down syndrome were retrieved as case group and 60 mothers of normal children as control group. The age of all mothers at the time of conception was less than 35 years and they were Han nationality.Genomic DNA was extracted from peripheral blood by using RelaxGene Blood DNA System kit. The genotypes of MTHFR $677 \mathrm{C} \rightarrow \mathrm{T}$, MTR $2756 \mathrm{~A} \rightarrow \mathrm{G}$, RFC-1 $80 \mathrm{~A} \rightarrow \mathrm{G}$ were detected by PCR-RFLP and all genetic polymorphisms were detected by GenomeLabSNP-Primer Extension Kit. The fast plasma homocysteine level was measured by HPLC with fluorescence. SPSS 15.0 assessed the statistical risk of these genotypes. Results. We found that frequence of MTHFR $677 \mathrm{C} \rightarrow \mathrm{T}$ mutation was more prevalent among morthers of children with DS than among the control group, with an OR of 2.60 (95\% CI 1.04-6.49). Moreover, the mutation of CBS $833 \mathrm{~T} \rightarrow \mathrm{C}$ was not found and no significant difference in other polymorphisms was observed between these two groups. The mean homocysteine concentration had no difference between case group $(8.51 \pm 2.55 \mu \mathrm{mol} / \mathrm{l})$ and control group $(8.41 \pm 1.42 \mu \mathrm{mol} / \mathrm{l})$. Conclusion. Maternal mutation of MTHFR $677 \mathrm{C} \rightarrow \mathrm{T}$ may be the risk factor for DS child without changing of homocysteine level in Han nationality.

\section{One Case Report of Idiopathic Syndrome of Inappropriate Antidiuretic Hormone Secretion and Review}

\section{Yi Gu, Chunxiu Gong, Jin Zhou, and Xuejun Liang}

\section{Beijing Children's Hospital, Capital Medical University}

Objective. Approach the clinical characteristic, diagnosis and treatmnet of idiopathic syndrome of inappropriate antidiuretic hormone secretion in order to improve the cognition and treatment of this disease. Method. Summarize one case of idiopathic inappropriate antidiuretic hormone secretion in Beijing Children Hospital, analyze clinical manifestation, laboratory examination, diagnosis and therapeutic method of this case combining with literatures. Meanwhile review literatures to gain relevant knowledge and progression of idiopathic inappropriate antidiure hormone secretion. Result. Clinical manifestations of this patient were respiratory infection with convulsion, severe polydipsia and diuresis greater than $3000 \mathrm{ml} / 24 \mathrm{~h}$ during the treatment.
Laboratory examination showed that sodium and osmotic pressure of blood serum decreased obviously. No significant effect with administration high tension sodium (5.5$8.5 \mathrm{mmol} / \mathrm{kg}$ ). Specific gravity and electrolyte excretion of urine increased obviously simultaneously. (sodium of blood serum $119.3 \mathrm{mmol} / \mathrm{L}$, osmotic pressure of blood serum 250 $270 \mathrm{mmol} / \mathrm{L}, \mathrm{SG} 1.030$, sodium of urine $920 \mathrm{mmol} / 24 \mathrm{hr}$, osmotic pressure of urine $626 \mathrm{mmol} / \mathrm{L})$. Finally, the restriction of water $1000 \mathrm{ml} / \mathrm{m}^{2}$ gained significant effect in this patient. MRI of head and pituitary were normal, excluding BSWS simultaneously. Diagnosis of this patient was idiopathic syndrome of inappropriate antidiuretic hormone secretion. The growth and development was normal in two years followup of this patient. Referring to the literature of domestic and abroad: little reports of inappropriate antidiuretic hormone secretion of children and no idiopathic inappropriate antidiuretic hormone secretion of children. Conclusion. The diagnosis of this patient was clinically based. Idiopathic inappropriate antidiuretic hormone secretion is very rare in pediatrics patients. It is easily neglected because of the insufficiecy cognition or its positive process by paediatricians. Differentiated from BSWS. This paper clues on that paediatricians should pay attention to discover and treat idiopathic inappropriate antidiuretic hormone secretion, with taking appropriate therapy of this disease.

\section{Cardiovascular Diseases in Turner Syndrome}

\author{
Jirina Zapletalova, ${ }^{1}$ Eva Klaskova, ${ }^{1}$ \\ Jaroslav Wiedermann, ${ }^{1}$ and Marta Snajderova ${ }^{2}$ \\ ${ }^{1}$ Department of Pediatrics, Palacky University and \\ University Hospital, Olomouc, Czech Republic \\ ${ }^{2}$ Department of Pediatrics, Charles University and \\ University Hospital, Prague-Motol, Czech Republic
}

Objective. To evaluate the prevalence of cardiovascular abnormalities in Turner syndrome (TS). Background. TS is the most common chromosomal abnormality, affecting 1/2000 live female birth. Congenital heart disease (CHD) affects approximately $50 \%$ of TS individuals and it is the major cause of premature morbidity and mortality in TS adults. Recent data report an association of TS with a generalised vasculopathy and aortic abnormalities such as aortic dilatation (AoDil) and dissection. The higher risk of $\mathrm{CHD}$ is associated with severe dysmorphic features. Bicuspid aortic valve (BAV), aortic coarctation (CoA), aortic root dilatation (ARD) and arterial hypertension $(\mathrm{AH})$ are the risk factors for aortic dissection. Risk of the aortic dissection is increased in young women particularly during pregnancy. Accurate data on prevalence of aortic valve abnormalities, AH and ARD are not available yet. Methods. Forty four individuals with TS (18 with karyotype 45, X) age 7.4 (0.1 to 19.0) years underwent complete cardiologic examination including ECG, echocardiography, 24 hours monitoring of arterial blood pressure and 38 of them also magnetic resonance imaging (MRI). The median of age at first cardiologic evaluation was $12.3(0.1-19.0)$ years. Results. BAV or dysplastic aortic valve was found in $29 \%$ of subjects, 
CoA was found in $2 \%$ of patients, aortic stenosis in $2 \%$ of subjects, AoDil in $18 \%$ and $\mathrm{AH}$ in $11 \%$ of subjects. Ascending/descending aortic diameter ratio $>95$ th percentile was found in $21 \%$ individuals. The cardiac abnormalities had $61 \%$ of all TS patients, but $78 \%$ of $45, \mathrm{X}$. Conclusion. One third of our patients had at least one risk factor for aortic dissection, but $44,5 \%$ in the group of $45, \mathrm{X}$ individuals. Our data confirm the high risk for cardiovascular diseases in TS. The patients should be carefully examined just after diagnosis of TS to detect the cardiovascular abnormalities and to identify many asymptomatic individuals with risk factors. Regularly followup by experienced cardiologist is necessary for early detection of cardiac abnormalities. MRI is helpful for early detection of AoDil in all TS girls at an age when the study may be performed without sedation to prevent cardiovascular morbidity and mortality.

\section{Trend in Overweight and Obesity Prevalence and Body Mass Index among Children and Adolescents Aged 6-18 Years Old in Two Districts of Shanghai}

Miao-Ying Zhang, ${ }^{1}$ Luo Fei-Hong Luo, ${ }^{1}$

Shui-Xiana Shen, ${ }^{1}$ Hong Xia, ${ }^{2}$ Yue-Zhen Tu, ${ }^{3}$

Feng-Xia Guo, ${ }^{4}$ Ting-Ting Huang, ${ }^{4}$ Di-Jing Zhi, ${ }^{1}$

Zhu-Hui Zhao, ${ }^{1}$ Rong Ye, ${ }^{1}$ Ruo-Qian Cheng, ${ }^{1}$

and Xiao-Jing $\mathbf{L i}^{\mathbf{1}}$

${ }^{1}$ Department of Endocrinology and Metabolism,

Children's Hospital, Fudan University, Shanghai 201102,

China

${ }^{2}$ Public Health Bureau, Minhang District,

Shanghai 201100, P. R. China

${ }^{3}$ Minhang District Center for Disease Control and

Prevention, Shanghai 201100, China

${ }^{4}$ Xuhui District Center for Disease Control and Prevention, Shanghai 200031, China

Background. It is unclear how is the trend of overweight and obesity prevalence and the trend of body mass index among children and adolescents in Shanghai. Objective. The aim of this survey was to establish baseline data on the trend in overweight and obesity prevalence and body mass index among children and adolescents in Shanghai. Design. We selected two districts in Shanghai in 2003, randomly, one in urban area and the other in suburb area. All students in the primary schools, junior and senior high schools of the two districts were screened in 2003 and 2008. Body composition was estimated by using BMI. Result. (1) The prevalence of overweight increased from $13.67 \%$ to $15.41 \%$ and the prevalence of obesity increased from $3.72 \%$ to $4.58 \%$ during the study periods. (2) The overall prevalence of obestiy and overweight in all children and adolescents (618 years) rose significantly between 2003 and $2008(P<.01)$. The standard prevalence of overweight and obesity increase significantly in each group $(P<.01)$. (3) The mean BMI was significantly higher in male students than that of female students $(P<.01)$. (4) Compared with data in 2003, the mean BMI of 2008 was significantly higher $(P<.01)$.
Conclusion. The prevalence of obesity and overweight in children and adolescents had increased over time in two districts of Shanghai. The distribution of BMI also showed differences between the sexes and between 2003 and 2008. 